\title{
Cerebrovascular Dysfunction in Metabolic Syndrome and Depression: Mechanisms and Implications for Neurovascular Disease
}

Steven David Brooks

Follow this and additional works at: https://researchrepository.wvu.edu/etd

\section{Recommended Citation}

Brooks, Steven David, "Cerebrovascular Dysfunction in Metabolic Syndrome and Depression: Mechanisms and Implications for Neurovascular Disease" (2016). Graduate Theses, Dissertations, and Problem Reports. 5261.

https://researchrepository.wvu.edu/etd/5261

This Dissertation is protected by copyright and/or related rights. It has been brought to you by the The Research Repository @ WVU with permission from the rights-holder(s). You are free to use this Dissertation in any way that is permitted by the copyright and related rights legislation that applies to your use. For other uses you must obtain permission from the rights-holder(s) directly, unless additional rights are indicated by a Creative Commons license in the record and/ or on the work itself. This Dissertation has been accepted for inclusion in WVU Graduate Theses, Dissertations, and Problem Reports collection by an authorized administrator of The Research Repository @ WVU.

For more information, please contact researchrepository@mail.wvu.edu. 


\title{
Cerebrovascular Dysfunction in Metabolic Syndrome and Depression: Mechanisms and Implications for Neurovascular Disease
}

\section{Steven David Brooks}

\begin{abstract}
Dissertation submitted to the School of Medicine at West Virginia University in partial fulfillment of the requirements for the degree of:
\end{abstract}

\section{Doctor of Philosophy in Cellular and Integrative Physiology}

\author{
Robert W. Brock, Ph.D., Committee Chair \\ Jefferson C. Frisbee, Ph.D.- Research Advisor \\ Timothy Nurkiewicz, Ph.D. \\ James Simpkins, Ph.D. \\ Paul Chantler, Ph.D. \\ William Mayhan, Ph.D., External Member \\ Cellular and Integrative Physiology Program \\ Morgantown, West Virginia \\ June, 2016
}

Key Words: Cerebrovascular disease, metabolic syndrome, endothelial dysfunction, ischemic stroke, depression, oxidative stress 


\section{ABSTRACT \\ Cerebrovascular Dysfunction in Metabolic Syndrome and Depression: Mechanisms and Implications for Neurovascular Disease}

\section{Steven D. Brooks}

Cerebrovascular diseases are any pathological conditions that are caused by disruptions or alterations in the blood supply to the brain. The brain, which is only $2 \%$ of body mass, constantly requires a minimum of $20 \%$ of cardiac output to meet its unique metabolic demands. Cerebral tissue relies on aerobic respiration and therefore requires a constant, steady supply of blood to provide oxygen and glucose to make ATP. Insufficient perfusion of the brain, which occurs as a result of reduced or obstructed blood flow, results in cerebral ischemia; ischemic neurons quickly deplete their available ATP and die if blood flow is not restored. Disruptions in the delivery of blood to the brain, either acutely or chronically, can lead to impairment of neurological function, neuronal cell death, and even death.

Metabolic Syndrome (MetSyn) is the comorbid presence of three or more risk factors, including: central obesity, hypertriglyceridemia, high cholesterol, hypertension, and hyperglycemia. Data from the NHANES study reports that over 35\% of all adults, and over $50 \%$ of adults over 60, are estimated to have MetSyn. MetSyn is a risk factor for almost every major cardiovascular disease, and causes well-defined impairments in the peripheral circulation. However, its effects on regulation of cerebral blood flow are not well understood. The primary aim of this dissertation is to study the functional and structural alterations cause by MetSyn in the cerebral circulation, utilizing the obese Zucker rat (OZR), a translationally relevant model for studying the vascular complications of MetSyn, and to interrogate how vascular and neurological outcomes to cerebrovascular disruptions are influenced by pre-existing cerebral vasculopathies associated with MetSyn in OZR. The specific aims are as follows:

1. Define and characterize structural and functional changes in vascular reactivity, vessel wall mechanics, and vascular remodeling in middle cerebral arteries (MCA) of OZR, and evaluate changes in microvascular density throughout the cerebral microcirculation.

2. Determine the contribution of each constituent pathology of MetSyn on the development of cerebrovascular impairments in OZR, and identify mechanisms through which these pathologies are progressing in the cerebral circulation.

3. Interrogate the mechanisms of stroke-induced vascular dysfunction in lean Zucker (LZR) and OZR, and determine how pre-existing vascular dysfunction in OZR changes outcomes and pathological mechanisms following ischemic stroke.

4. Investigate the effects of chronic stress/ depression on cerebrovascular function in LZR and OZR, assess efficacy of exercise as a prophylactic anti-depressant treatment, and evaluate mechanisms through which impairments, and exercise-based recovery, are occurring.

The results of these studies have helped to establish the prominent driving mechanism of cerebrovascular dysfunction in MetSyn, and defined the functional impacts these impairments have on outcomes to ischemic stroke and stress-induced depression. By thoroughly interrogating these mechanisms and evaluating functional outcomes of relevant disease states, this dissertation hopes to lay the groundwork for a new approach to understanding the relationship between MetSyn, cerebrovascular dysfunction, and neurological outcomes. 


\section{Dedication}

To Mom, Dad, Daniel, Michael, and Matthew

To my family, friends, and advisors who showed me true patience as I struggled to find my path.

To everyone at the WVU HSC whom I have crossed paths with over the last 5 years, who brightened my days when I needed it most. 


\section{Acknowledgments}

The work presented here within this dissertation, and indeed almost everything I have worked on over the last five years, has been influenced, discussed, amended, and adjusted by 100 different people. I don't believe that anyone can ever lay an exclusive claim to an idea, a fact, or the truth, so I would like to acknowledge every colleague, every professor, every student, and every stranger who I have ever talked science with. Your impact is reflected in these pages.

I want to sincerely thank my family, who has supported me in innumerable ways as I blundered my way into graduate school, starting with my failed foray in the $\mathrm{MD} / \mathrm{PhD}$ program, transitioning into the neuroscience and finally the physiology program. My plans may have changed a dozen times, but their love and support did not. My family is the definition of unconditional love, support, and patience, and I can never thank them enough.

Special thanks to Dr. Fred Minnear, who reached out to me when I was lost and ready to quit on academia while I was in the $\mathrm{MD} / \mathrm{PhD}$ program. Dr. Minnear gave me a lifeline, helped me transition into a career path that was a better fit for me, and then pushed me hard to excel.

I would like acknowledge Dr. Taura Barr, who was my first real scientific mentor. Taura introduced me to idea of translational science, and her influence and approach to the scientific process are reflected in the way I approach my work. Fate often picks a winding path for us, and I was lucky to have Taura guide me part of the way. Plus, she took me to Hawaii.

I would like to thank my mentor, Dr. Jefferson Frisbee, for everything he has done for me over the past three years. Like many others before me, Jeff took me in when I was academically homeless, and proceeded to transform me into someone new. Studying under Jeff exposed me to a different level of intellectual rigor, helped me get an AHA fellowship, and has opened more doors professionally than I could ever fully count. I can say without hesitation that I very much doubt I would have ever made it anywhere in this field without Jeff's mentorship. 
To the fellow members of the Frisbee laboratory- you guys are/were the best. Trey, Shyla, Josh- you each know how I feel about you. And Josh- you literally taught me everything I know about working with animals and vessels. Also, how to properly enjoy lunch breaks, how to network at meetings, and how never take life too seriously, no matter what it throws at you. I hope I can be half the mentor that you were to me, and a quarter the optimist. Also, big shout out to my undergraduate students- Paulina, Camille, and Whitney (Jordan!). You guys did the dirty work, and for that I am eternally grateful. I hope you enjoyed it at least a little, and don't forget about us researchers when you've got a shiny MD or DO after your name.

I would like to thank Dr. Paul Chantler, and everyone in the Chantler Lab- Evan, Shin, Sara, Kayla, Kent, Chris- you guys are co-authors on these chapters for a reason. Working with you has been the definition of collaborative, team science, and I wouldn't trade it for the world. I wish you all the best and hope to always keep in touch. 


\section{Table of Contents}

Cerebrovascular Dysfunction in Metabolic Syndrome and Depression: Mechanisms and Implications for Neurovascular Disease

Abstract

$\begin{array}{ll}\text { Dedication } & \text { iii }\end{array}$

Acknowledgements $\quad$ iv

Table of Contents $\quad$ vi

Abbreviations $\quad$ vii

Chapter 1:

Review of the Literature 1

Chapter 2:

Cerebral Cortical Microvascular Rarefaction in Metabolic Syndrome is Dependent on Insulin

Resistance and Loss of Nitric Oxide Bioavailability

Chapter 3:

Metabolic Syndrome Impairs Reactivity and Wall Mechanics of Cerebral Resistance Arteries in

Obese Zucker Rats

Chapter 4:

Oxidative Stress Exacerbates Vascular Dysfunction and Infarct Size after Ischemic Stroke in a Rodent Model of Metabolic Syndrome

120

Chapter 5:

Exercise Training Protects Against Chronic Stress/Depressive Behavior Induced Cerebrovascular Dysfunction and Cortical Rarefaction in Lean and Obese Zucker Rats

Chapter 6:

Dissertation Discussion

Appendix:

An Unpredictable Chronic Mild Stress Protocol for Instigating Depressive Symptoms, Behavioral Changes and Negative Health Outcomes in Rodents 


\section{Abbreviations}

4-Hydroxy-Tempo

(TEMPOL)

5-Hydroxytryptamine

(5-HT; Serotonin)

Acetylcholine

$(\mathrm{ACh})$

Blood Brain Barrier

$(\mathrm{BBB})$

Cardiovascular Disease

(CVD)

Endothelial Nitric oxide synthase

(eNOS)

Lean Zucker Rat

(LZR)

$\mathrm{L}-\mathrm{N}^{\mathrm{G}}$-nitroarginine methyl ester

(L-NAME)

Metabolic Syndrome

(MetSyn)

Middle Cerebral Artery

(MCA)

Mild Cognitive Impairment

(MCI)

Nicotinamide Adenine Dinucleotide Phosphate

$(\mathrm{NAD}(\mathrm{P}) \mathrm{H})$

Nitric Oxide

(NO)

Obese Zucker Rat

$(\mathrm{OZR})$

Prostacyclin

$\left(\mathrm{PGI}_{2}\right)$

Reactive Oxygen Species

$(\mathrm{ROS})$

Sodium Nitroprusside

$(\mathrm{SNP})$

Tissue Plasminogen Activator

(tPA)

Transient Ischemic Attack

(TIA)

Unpredictable Chronic Mild Stress

(UCMS) 


\section{CHAPTER 1: REVIEW OF THE LITERATURE}

\section{Part 1: Introduction to Cerebrovascular Disease}

Cerebrovascular diseases are any pathological conditions that are caused by disruptions or alterations in the blood supply to the brain [1]. The brain, which is only $2 \%$ of body mass, constantly needs $20 \%$ of cardiac output to meet its unique metabolic demands [2, 3]. Cerebral tissue does not store glucose, and relies principally on aerobic respiration, so the brain requires a constant, steady supply of blood to provide the necessary oxygen and glucose needed to make ATP $[4,5]$. Insufficient perfusion of the brain, which occurs as a result of reduced or obstructed blood flow, results in cerebral ischemia[6]; ischemic neurons quickly deplete their available ATP and will rapidly die if blood flow is not restored[3, 5]. Such disruptions in the delivery of blood to the brain, either acutely or chronically, can lead to impairment of neurological function, neuronal cell death, and even death [7].

Acute cerebrovascular diseases, such as ischemic stroke and transient ischemic attack (TIA), occur as a result of a sharp decrease in blood flow to a region of the brain, causing an area of cerebral tissue to become ischemic $[1,3,8]$. The most common etiology of acute ischemia is arterial blockage by a blood clot, but can also result from hypoperfusion due severe hypotension or cerebral hemorrhage $[3,7]$. Neurons have a critical oxygen tension of $25-40 \mathrm{mmHg}$; when $\mathrm{P}_{2}$ drops below this threshold, aerobic production of ATP can decline by over $90 \%$ within 5 minutes [4]. Neurons depend on a constant supply of ATP, and cannot produce ATP via anaerobic respiration in sufficient amounts to survive. Within minutes of the ischemic onset, ATP starvation will cause hypoxic neurons to depolarize, allowing an influx of $\mathrm{Ca}^{++}$that triggers ejection of neurotransmitters like glutamate, and lysis of the cell, releasing large amounts of reactive oxygen species (ROS) $[4,9,10]$. Glutamate binds neighboring NMDA receptors, exiting 
these neurons and stimulates an excitatory injury through neighboring neurons, and oxidative damage inflicted by ROS can induce apoptosis in these neighboring cells as well [4, 9]. The ischemic core of infarcted neurons will die as a result of the acute hypoxia; however, the spreading nature of the excitotoxic and oxidative damage can induce necrosis and apoptosis of neurons in neighboring neurons that were otherwise adequately perfused, an area of at-risk neurons known as the penumbra $[6,9,11]$ The physical symptoms that define stroke and TIA, such as facial dropping, impaired motor control/weakness of the limbs, and slurred or difficult speech, are caused by the immediate dysfunction of hypoxic neurons. If the hypoxic neurons become necrotic, the symptoms may become permanent long-term disabilities $[6,8,11,12]$. However, during a TIA, the hypoxic neurons recover due to rapid restoration of blood flow, and no infarction or penumbra will develop. The neurological symptoms will resolve, and no permanent lasting damage occurs [7, 13].

The principal difference between ischemic stroke and TIA is the ability of the patient's body to quickly restore blood flow to the ischemic area [13]. Time is the most crucial factor in this equation, as reflected by the American Stroke Association's mantra of "Time is Brain" [12]. Mechanistically, the ability to restore blood flow involves rapid dissolution of the blood clot and/or diversion of blood into the ischemic region via collateral vessels $[6,7,12]$. Restoration of blood flow is also critical in enhancing the survival of the at-risk neurons populating the penumbra $[14,15]$. The most active areas of stroke research therefore have historically centered on ways to lyse or remove clots, improve collateral blood supply, reduce the spread of excitotoxic injury, or prolong the ability of hypoxic neurons to survive and therefore lengthen the gap between onset of hypoxia and infarction $[7,11,12,14,15]$. 
Figure 1: Schematic Representation of Ischemic Stroke

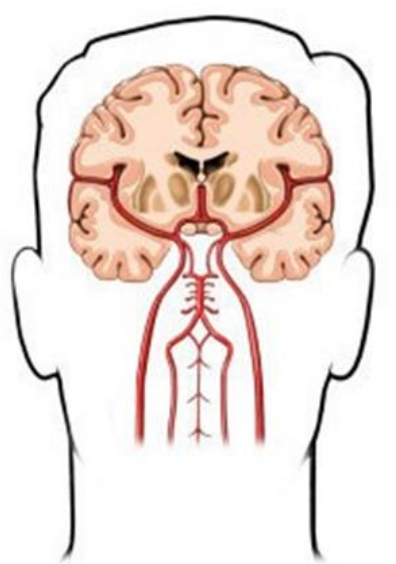

Cerebral Blood Supply

Figure Adapted From Creighton University SOM Stroke Program

Chronic cerebrovascular disease

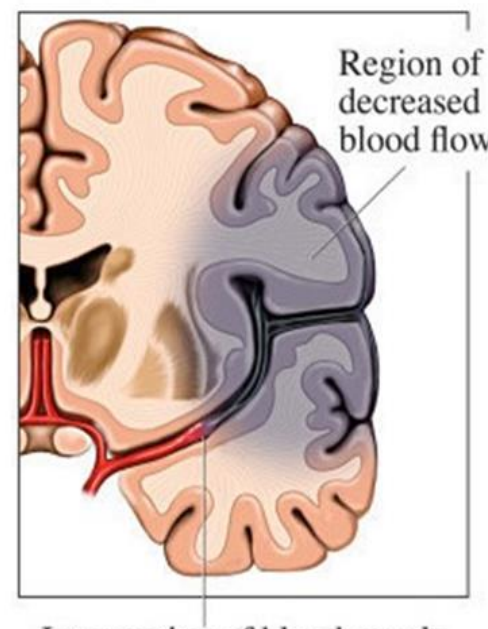

Interruption of blood supply

mechanisms vary by patient, but the general result is loss of mental ability including clarity of executive thought (mild cognitive impairment, or MCI) $[3,17,18]$, impairments to memory formation and memory recall, and gradual impairments in motor and sensory function $[1,3,19]$. The most prominent chronic cerebrovascular disease is dementia, which includes both Alzheimer's and vascular dementia $[3,16,20]$. Both vascular dementia and MCI are characterized clinically by the presence of small infarcts throughout the brain, often observable via MRI [17, 21]. While each small infarct on its own may have not caused noticeable symptoms, their accumulation over time results in cognitive impairments and symptoms of dementia [21, 22].

A third type of cerebrovascular disease is intracranial aneurysm. An intracranial aneurysm is a disorder in which a weak or malformed cerebral artery balloons out due to its structural weakness, and may eventually rupture, leading to intracranial hemorrhage $[215,216]$. The largest and most common intracranial aneurysm is the saccular aneurysm, which typically arise in or immediately adjacent to the Circle of Willis [217]. The etiology of saccular aneurysms are almost always hereditary [215, 218], and therefore do not neatly fit the definition of acute or 
chronic cerebrovascular disease as defined here. Because of its genetic components, aneurysm will not be included or discussed alongside the other cerebrovascular diseases listed in this dissertation. Cardiovascular risk factors, such as hypertension and smoking, can increase risk of aneurysm rupture and vasospasm [215, 218]; however, these mechanisms are common to the pathways through with $\mathrm{CV}$ risk factors affect cerebrovascular circulation in general. For simplification and topicality, the discussion of these mechanisms on post-aneurysm outcome will be omitted as well.

While both ischemic stroke and cognitive vascular diseases represent distinct pathological conditions, they share a common etiology rooted in the dysregulation of cerebral blood flow [23, 24]. This review will present the structure and regulation of the cerebral circulation in the condition of health, and then explore how the individual components of the cerebral vasculature malfunction under pathological conditions. As both ischemic stroke and vascular cognitive impairments are rooted in underlying vascular comorbidities, it is important to first characterize the nature of cerebrovascular dysfunction in the metabolic syndrome, a prevalent condition that is strongly linked as a risk factors for stroke and vascular dementia due to its constituent underlying pathologies [25-29]. The mechanisms of vessel function and blood flow regulation during ischemic stroke will then be detailed, with emphasis on differences between healthy and cardiovascular disease (CVD) comorbid studies. Lastly, the mechanisms of cerebrovascular dysfunction and cognitive impairments will be discussed. By highlighting the mechanisms and identifying commonalities between these conditions, this reviews aims to establish a robust framework from which to further explore how mechanisms of cerebrovascular dysfunction impact highly relevant health outcomes in several common disease states. 


\section{Part 2: Public Health and Disease Burden of Cerebrovascular Disease and Metabolic}

\section{Syndrome}

Ischemic Stroke is among the leading causes of death and disease burden worldwide. According to the AHA's 2016 Heart Disease and Stroke Statistics update, around 800,000 people will experience a stroke every year in the US; a stroke occurs on average every 40 seconds, and approximately 15 people die every hour from stroke [30, 31]. While overall mortality has improved in the past 6 years, the prevalence of stroke has not decreased, and the total number of strokes is expected to double by 2050 as the population continues to age. Stroke is therefore one of the leading causes of serious long-term disability and decreased quality of life, and alone is responsible for a disease cost burden of over $\$ 34$ billion in the US annually $[8,12,30]$. Stroke also disproportionately affects the most vulnerable and disadvantaged of our population, as stroke death rates are highest in African Americans and Hispanics, and also greatly elevated in Appalachia and throughout the southeastern US [8, 30,31].

Despite the prevalence of ischemic stroke, treatment options for patients are very limited. Currently, there are only two FDA-approved treatments for ischemic stroke [7, 12]. The "gold standard" treatment is intravenous recombinant tissue plasminogen activator (IV tPA), first approved for use in 1996, which works to dissolve the blood clot and restore blood flow to the ischemic area $[12,32]$. However, IV tPA usage is restricted by a narrow treatment window ( 0 to 3-4.5 hours after symptom onset), as well as contra-indicated for use in many patients due to elevated hemorrhage risk. As a result, IV tPA is vastly under-utilized, with estimates of usage ranging from only $3 \%-7.0 \%$ of stroke patients [32-34]. The second most common treatment for stroke is endovascular surgery, in which a clot is either retrieved or directly aspirated with tPA 
$[7,12,34]$. Usage of endovascular treatment for stroke is low, however, due to the specialization of the procedure and the stringent inclusion criteria for treatment.

While stroke research has been a priority of both national funding agencies and pharmaceutical companies, no new drugs have been approved since tPA first gained approval in 1996. Intravenous tPA's benefit is solely derived from its ability to more quickly restore perfusion, and many other drugs have been sought to improve this strategy [15, 35]. Other strategies have focused on improving collateral blood flow to ischemic areas, enhancing the ability of neurons to survive under hypoxia to extend the effective window for reperfusion-based recovery, and limiting the spreading excito-toxic effects that often cause larger infarctions during ischemic stroke [36, 37]. Clearly, novel strategies are needed to expand treatment options and efficacies for stroke patients, and chapter 4 of this dissertation will addresses such an approach.

The US Centers for Disease Control names high blood pressure, high cholesterol, heart disease, and diabetes as significant independent risk factors for stroke, with hypertension being the most significant $[8,38]$. The metabolic syndrome, which encompasses each of these risk factors as constituents, is also itself a significant risk factor for stroke [38-40]. The metabolic Syndrome is defined as the presence of three or more risk factors, including: central obesity, hypertriglyceridemia, high cholesterol, hypertension, and hyperglycemia [25, 26, 38]. Data from the NHANES study reveals that over $35 \%$ of all adults, and over $50 \%$ adults aged 60 or older, are estimated to have the metabolic syndrome [25]. The conditions that underlie the metabolic syndrome, including most types of cardiovascular disease, have a cumulative cost of over $\$ 300$ billion in the US annually, which comprises the largest financial burden on the US healthcare system. For contrast, the annual cost of all types of cancer is just under $\$ 90$ billion $[25,26]$. 
Treatment of patients with the metabolic syndrome focuses on managing and reducing the individual risk factors themselves $[25,26]$. Notably, however, each of these risk factors has a distinct impact on the regulation and delivery of blood flow [41-44]. Each constituent pathology is linked to impairments arterial function, and the resultant restrictions in blood flow lead to increased incidence of heart attack and stroke [41, 45, 46]. Studying these underlying mechanisms, and how they interact with each other, can provide valuable insight into the progression of vascular disease, and help identify potential areas for therapeutic development. The relevant pathophysiological mechanisms that underlie the metabolic syndrome, and their specific effects in the cerebrovasculature, will be detailed in the fourth part of this introduction.

Chronic impairments in blood flow delivery to the brain often manifest as cognitive impairments $[17,21]$. The CDC estimates that over 16 million adults currently suffer from some form of cognitive impairment, ranging from Mild Cognitive Impairment (MCI) to more advances dementias, such as Alzheimer's disease and vascular dementia [47]. The direct and indirect costs of caring for these patients as their symptoms worsen has been estimated as high as $\$ 144$ billion annually, and is expected to rise as the population continues to age [16, 21, 48, 49]. Currently, no FDA-approved treatments are available to treat or reverse these cognitive conditions; only drugs which slow the onset are available. While not all dementia or cognitive impairments are strictly vascular-derived, blood flow related issues are thought to be amongst the most common underlying factors, and patients with heart disease, stroke, and other types of CVD are all at elevated risk of developing cognitive impairments later in life [17, 22, 29, 31, 48-50]. 
Table 1: Lifetime Prevalence of Stroke, Dementia, and other Cerebrovascular Pathologies among all US adults age 60 or older

\begin{tabular}{|c|c|c|c|}
\hline \multicolumn{4}{|c|}{ Lifetime Prevalence of Cerebrovascular Disease in the United States } \\
\hline & Adults (>60 years) & Men (>60 years) & Women (>60 years) \\
\hline $\begin{array}{c}\text { Ischemic Cerebrovascular } \\
\text { Events }\end{array}$ & $\mathbf{2 2 \%}$ & $\mathbf{1 7 \%}$ & $\mathbf{2 1 \%}$ \\
\hline Ischemic Stroke & $18 \%$ & $15 \%$ & $19 \%$ \\
\hline Transient Ischemic Attack (TIA) & $3 \%$ & $2 \%$ & $3 \%$ \\
\hline $\begin{array}{c}\text { Mild Cognitive Impairment } \\
\text { (MCI) }\end{array}$ & $\mathbf{2 0 \%}$ & $\mathbf{1 8 \%}$ & $\mathbf{2 0 \%}$ \\
\hline All Dementia & $\mathbf{1 6 \%}$ & $\mathbf{1 3 \%}$ & $\mathbf{1 9 \%}$ \\
\hline Alzheimer's Disease & $11 \%$ & $10 \%$ & $17 \%$ \\
\hline Vascular Dementia & $4 \%$ & $\sim 4 \%$ & $\sim 4 \%$ \\
\hline Stroke or Dementia & $\sim 40 \%$ & $\mathbf{3 5 \%}$ & $\mathbf{5 0 \%}$ \\
\hline Sources: [16, $17,31,40,48,49,51]$ & & & \\
\hline
\end{tabular}

Table 2: Prevalence of Metabolic Syndrome and Depression in US Adults

\begin{tabular}{|c|c|c|c|}
\hline \multicolumn{4}{|c|}{ Lifetime Prevalence of Metabolic Syndrome and Depression in the US } \\
\hline & All Adults & Men & Women \\
\hline Metabolic Syndrome (All Adults) & $\mathbf{3 5 \%}$ & $\mathbf{3 7 \%}$ & $\mathbf{3 3 \%}$ \\
\hline Metabolic Syndrome (>60 years) & $\mathbf{5 0 \%}$ & $\mathbf{4 4 \%}$ & $\mathbf{5 4 \%}$ \\
\hline All Depressive Mood Disorders & $\mathbf{2 1 \%}$ & $\mathbf{1 5 \%}$ & $\mathbf{2 7 \%}$ \\
\hline Major Depressive Disorder & $\mathbf{1 6 \%}$ & $\mathbf{8 - 1 2 \%}$ & $\mathbf{2 0 - 2 6 \%}$ \\
\hline Sources: $[25,27,39,52-56]$ & & \\
\hline
\end{tabular}

Depression is another independent risk factor for development of MCI and dementia [20, 54, 57, 58]. Interestingly, depression is also an established risk factor for developing CVD, and inversely, CVD is an independent risk factor for developing depression [59-67]. While a strong causal mechanism defining the relationship between depression and CVD is unknown, depression has been shown to have direct effects on vascular function. Patients with depression exhibit impaired flow-mediated dilation and endothelium-dependent dilation, as well as 
decreased plasma nitric oxide (NO) and endothelial nitric oxide synthase (eNOS) activity [60, 68-70]. Additionally, obesity, a major CVD risk factor and component of the metabolic syndrome, is also directly associated with depression $[68,71,72]$. As over $15 \%$ of adults will suffer from depression $[53,54,58,73,74]$, a large number of patients will suffer comorbidity between depression and CVD, depression and the metabolic syndrome, and depression and stroke $[55,58]$. A potential linking pathway between these conditions may involve regulation of the Sympathetic-Adrenal-Medullary (SAM) axis and the hypothalamic-pituitary-adrenal (HPA) axis $[68,75-79]$; chronic stress, which is among the most well-established etiological triggers for depression, is known to cause hyper-activation of the SAM and HPA axes, leading to enhanced catecholamine release, elevated glucocorticoid levels, increased chronic inflammation, hyperglycemia, and risk of oxidative stress $[61,65,71,80,81]$.

The HPA axis modulates the neuroendocrine stress response by stimulating production of glucocorticoids, such as cortisol, and activating the SAM axis to ready the fight-or-flight response. However, chronic stress causes long term elevation of stress hormones, which overwhelm the endogenous negative feedback mechanisms of the hypothalamus and pituitary $[65,79,82-87]$. Clinically, hypercortisolemia stemming from HPA axis over-activation [88] is one among the most common biomarkers found in depressed patients [89, 90]. Cortisol dysregulation has direct consequences for vascular health, contributing to impairment of vasomotor responses and promoting endothelial dysfunction [66, 86, 88, 90, 91]. Glucocorticoids can directly reduce endothelial NO bioavailability by limiting tetrahydrobiopterin (BH4) and eNOS gene transcription, and also by affecting pathways of reactive oxygen species production $[76,86]$, and studies that have found treatment with an inhibitor of glucocorticoid production results in improved endothelial dysfunction in depressed patients [92, 93]. Chronic 
hypercortisolemia also promotes low-grade, chronic inflammation [94, 95], and cortisol insensitivity develops in lymphocytes of depressed patients [91, 94], impairing their ability to attenuate immune responses and leading to chronic inflammation [95]. Hyperactivity of SAM axis is also observed in depressed patients, causing an increase in catecholamine hormone release [96-98] [e.g. norepinephrine (NE) and epinephrine (Epi), as well as the monoamine serotonin (5HT)] that is associated with several CVD risk factors, including congestive heart failure [97, 99102] and diabetes [103]. In the peripheral circulation, these hormones innervate vascular smooth muscle cells and endothelial cells and alter vascular reactivity [43, 98, 104-106].

Figure 2: Pathways Linking Chronic Stress and Depression with Vascular Dysfunction

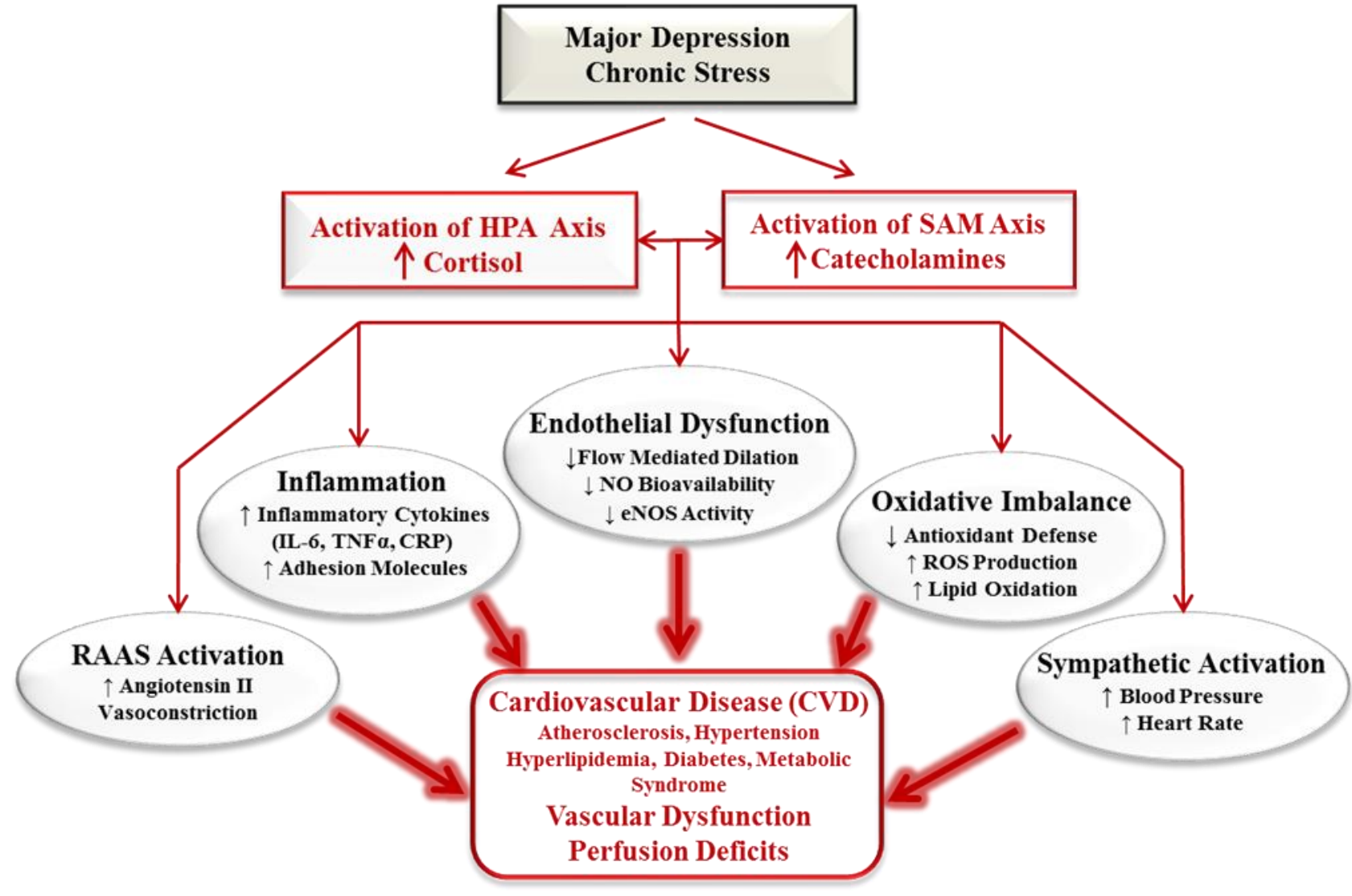

Each subset of cerebrovascular disease is characterized by distinct pathophysiological pathways that impact the function of the vascular endothelium, vascular smooth muscle, and/or 
cerebral vessel mechanics. Specific examples of these impairments have been previously identified in hypertension, hypercholesterolemia, diabetes, the metabolic syndrome, and depression $[2,3,22,23,36]$. Impaired regulation of cerebral blood flow has clear implications for prevention, acute treatment, and long-term recovery in patients at risk of ischemic stroke, and improved cerebral blood flow may have clear implications for patients with depression, or at risk of developing cognitive impairments later in life [24, 27, 45, 107]. This aim of this review is to examine the known mechanisms of cerebrovascular dysfunction inherent in CVD and in cerebrovascular disease, in order to better identify and define a vascular linkage between each of them.

\section{Part 3: The Arterial Components of the Cerebral Circulation-Structure, regulation, and}

\section{mechanisms of function in health}

The cerebral circulation is the unique and critical system through which the body supplies the brain with nutrients and regulates perfusion to the different regions of the brain based on metabolic demand. The brain, which represents only approximately $2 \%$ of total body mass, is comprised of some of the body's most constitutively active tissue in terms of oxygen and glucose consumption, and therefore consistently receives $15-20 \%$ of the blood flow from the heart [2, 108]. The arterial component of the cerebral circulation has a unique anatomy to help ensure

constant blood flow to the brain, and given its critical importance, contains inherent redundancies governed to ensure adequate blood flow $[2,3,45,108]$. The arteries and arterioles of the cerebrovasculature are also uniquely regulated, with extrinsic, intrinsic, and autoregulatory mechanisms exerting different levels of control, depending on the size and function of the vessel. 


\section{Anatomy of the Cerebrovasculature}

The main conduit arteries supplying the cerebral circulation are the left and right internal carotid arteries, and the basilar artery, which arises when the two vertebral arteries coalesce into a single conduit along the brain stem. These three large arteries each merge into a looping network of communicating arteries, known as the circle of Willis, providing excellent redundancy of blood flow $[2,107]$. The majority of the blood that circulates throughout the cerebrum comes from the two internal carotids, while the basilar artery also serves as a branch point for the main arteries supplying the cerebellum and brain stem. However, the anastomotic ring structure of the circle of Willis ensures distribution of blood flow from all three major conduits is maintained throughout the brain, so that reduction of loss of blood supply from either of the carotids or the basilar artery is compensated completely by blood supply from the other two $[2,107,109]$.

\section{Figure 3: Arterial Circulation of the Brain}

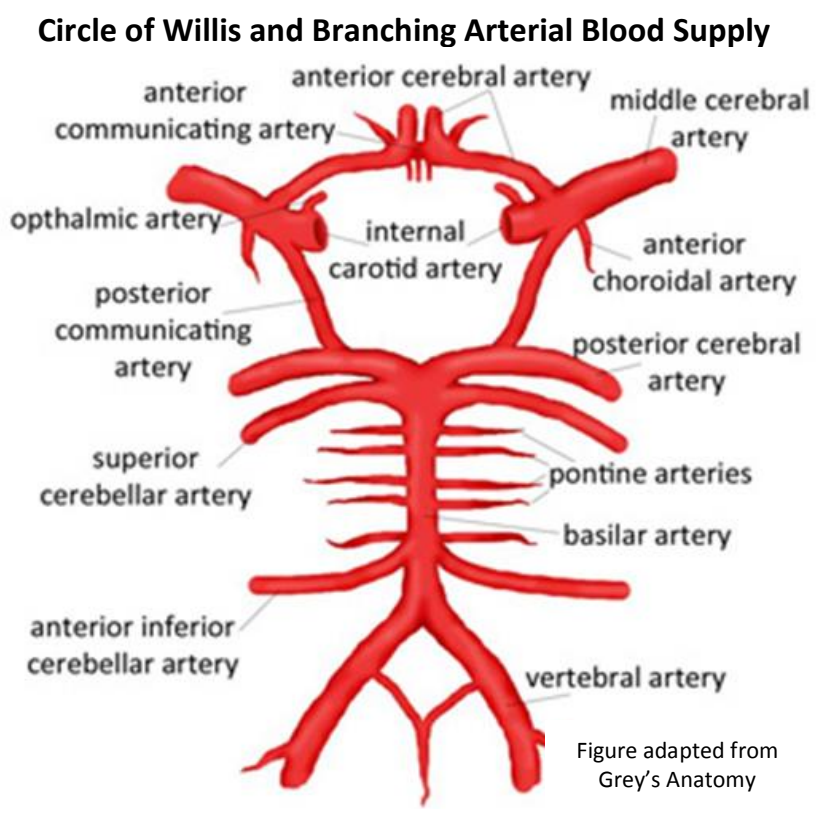

Branching from the circle of Willis, there are three main hemispheric pairs of large pial (surface) arteries; the anterior, middle, and posterior cerebral arteries (ACA, MCA, and PCA respectively); that form that beginning of branching arterial networks supplying blood to each region of the brain. These pial arteries each progressively split into smaller daughter

arteries, which lay across the exterior surface of the brain in the pia-arachnoid space or within the glia limitans $[2,110-112]$. Pial arteries are surrounded by cerebrospinal fluid (CSF), and 
narrow as they become more distal to the circle of Willis. Redundancy of blood flow is again observed in the spatial alignment of the pial arteries, which are located in close enough proximity to be capable of supplying overlapping perfusion territories of sister arteries with blood flow. This phenomenon is known as collateral blood supply; functionally, blockages or reductions in flow of a single pial artery can often be compensated for by the neighboring pial arteries [2, 109]. Penetrating arterioles branch off of the pial arteries and begin the descent into the cortex, occupying spatial invaginations known as the Virchow-Robin space. Once the penetrating arterioles enter the cortical tissue, they continue to narrow and form into the smallest resistance arterioles of the brain, called parenchymal arterioles [2, 110, 113]. In contrast to pial arteries, parenchymal arterioles are almost completely surrounded by astrocytes, a hallmark feature of the cerebral blood-brain barrier $(\mathrm{BBB})$, and reflective of their tight regulation by the surrounding neuropil. Lastly, these parenchymal arterioles perfuse a highly interconnected network lattice of capillaries, supplying blood throughout the cerebrum [2, 110, 113].

Each artery and arteriole of the cerebrovascular circulation is composed of three concentric layers- the tunica intima, comprised of an endothelial cell layer and the internal elastic lamina; the tunica media, comprised of vascular smooth muscle cells; and the tunica adventitia, comprised of a collagen fiber network, peripheral or perivascular nerve termini, fibroblasts, and/or astrocytic end feet $[2,107,108]$. The endothelial cell layer in these cerebral vessels is unique compared to endothelium in most other vascular beds, and forms the bedrock of the BBB. Each endothelial cell is joined to its neighbors by specialized tight junctions which greatly limit diffusion and transport from the blood into the cerebral interstitial fluid $[114,115]$. The BBB also includes the cerebral arteries and arterioles, and their surrounding astrocytic end feet which provide biochemical support and moderate control of diffusion between neuronal tissue and the 
cerebrovasculature $[2,45,108]$. Despite structural similarities, however, pial arteries, penetrating arterioles, and parenchymal arterioles each have varying structural and regulatory components that inform their different functions in regulating cerebral blood flow.

Conduit and pial arteries have multiple layers of vascular smooth muscle cells, ranging from 20 layers thick in the internal carotids down to 3 layers thick in the smallest pial arteries [2, 112, 116]. The well-developed smooth muscle layer allows the pial artery network to significantly regulate bulk blood flow by controlling resistance, making the cerebrovasculature unique from most peripheral vascular beds, in which resistance is more highly impacted by smaller arterioles. Pial arteries are extrinsically innervated by the nervous system, with neurotransmitters such as Nitric Oxide (NO), acetylcholine (ACh), norepinephrine (NE), and serotonin (5-hydroxytryptophan, or 5-HT) each having vasoactive effects on vessel diameter. They are also responsive to circulating factors $[2,108,112]$. Mechanisms of vascular regulation will be discussed in greater detail in the next section.

The penetrating and parenchymal arteries exhibit only a single layer of vascular smooth muscle cells, and are not extrinsically innervated by perivascular nerves. Instead, these arterioles are intrinsically innervated by the neurons and glial cells in which they are embedded $[2,116]$. In further contrast to pial arteries, penetrating and parenchymal arterioles exhibit vastly different responses to neurotransmitters (e.g. NE and 5-HT), and possess higher levels of basal vascular tone $[109,117,118]$. Metabolic and endothelial factors also play an important role in regulation of these vessels. In contrast to pial arteries, penetrating and parenchymal arterioles do not typically branch laterally. Occlusions or blockages are not easily compensated by collateral flow, and therefore, narrowing or occlusion of these vessels frequently leads to ischemia and infarction of the immediate surrounding cerebral tissue [109]. 
Figure 4: Schematic Representation of Cerebral Arteries and Arterioles

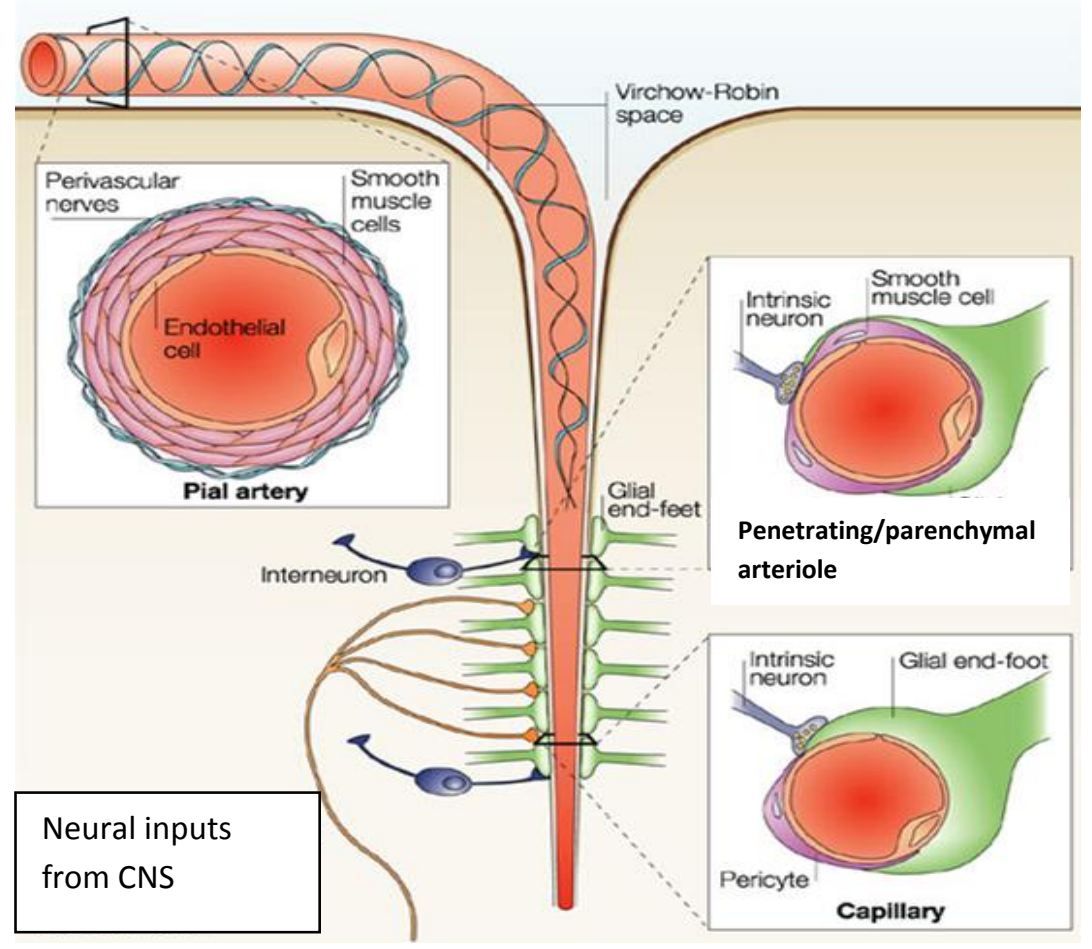

Image adapted and modified from ladecola 2004, Nature Neuroscience Reviews
The parenchymal arterioles give birth to the capillary beds of the cerebral circulation. Typical of capillaries, these vessels are composed of a single endothelial cell layer and have no smooth muscle cell layer [2, 108]. In contrast to peripheral capillary beds, the

endothelium of cerebral capillaries forms the distinct BBB, and the capillaries are all always open and perfused. Perfusion pressure within the capillaries is governed by flow from the feeding arterioles; as arterioles dilate, perfusion pressure increases, driving increased capillary flow $[112,116]$. The brain is extraordinarily rich in capillarity, with some estimates stating that every neuron may have its own capillary. The proximity and importance of blood flow to each neuron lends further credence to the hypothesis that regulation of cerebral blood flow may be directly linked to the performance of neurons at the individual and population level [119].

Cerebral capillary density is also variable depending on environmental or pathological stimuli. Growth and development result in neovascularization, with the density of cerebral capillaries increasing in response to rising activity levels in the brain [2, 120-122]. Chronic hypoxia also activates angiogenic pathways, regulated by VEGF and HIF-1, which increase 
capillarity in the brain [123]. On the other hand, pathological conditions such as hypertension have been linked with rarefaction of cerebral microvessels $[124,125]$, as well as with impaired angiogenic pathways which hamper the formation of new arterioles and capillaries [120, 121]. In other disease states, such as diabetes, the effect is less clear. Dr. Fagan's research team reports that type 2 diabetic rats display increased neovascularization in the cerebral cortex $[33,121,122$, 126], but that these new vessels were often non-viable or non-perfused, resulting proliferation of small vessels but functionally demonstrating perfusion deficits.

The mechanisms of endothelial dysfunction and oxidative damage discussed in this dissertation focus on the arterial circulation; however there are important differences between the cerebral venous system and the peripheral venous system that should be briefly discussed. The cerebral venous system is comprised of larger superficial veins and dural sinuses, and deep central veins which drain the white matter and deeper brain structures [2, 219, 220]. In contrast to peripheral veins, cerebral veins lack valves and do not have a tunica media layer $[2,219,220]$. They are mostly imbedded in the dura mater, protecting them from compression, and due to their lack of tunica media, the cerebral veins remain perpetually dilated to enhance drainage into the jugular veins $[219,220]$. An additional difference is that the cerebral veins form a tight blood brain barrier $[219,221]$, similar to the cerebral arterial side. While peripheral veins exhibit varying levels of permeability, and are the primary site of leukocyte invasion of tissue, the BBB of the cerebral veins is known to prevent these events in the cerebral circulation [221, 222]. Some evidence shows that in conditions of chronic disease and inflammation, BBB breakdown may lead to some permeability of cerebral veins [223]; otherwise, cerebral veins, like their arterial counterparts, protect the brain from direct interaction with the leukocytes of the systemic immune system [221, 222]. In terms of thrombosis, Virchow's Triad stipulates clot formation is 
most likely in states of 1) hypercoagulability, 2) venous stasis, or 3) endothelial injury/dysfunction [224]. In comparing the periphery with cerebral venous circulation, condition 1 is universal and related mostly to circulating clotting factors. Condition 2, however, will differ between central and peripheral veins, in that the lack of valves and structural protection of vertebral veins helps prevent venous stasis $[2,222,224]$. Lastly, until very recently, it was not believed that the brain possessed any lymphatic drainage [225]; this discovery has revolutionized our understanding of lymphatic clearance from the brain, but is yet to be well-characterized.

\section{Regulation of the Cerebrovasculature}

Given the brain's substantial basal metabolic rate, and the ubiquitous perfusion of all arterioles and capillaries, precise regulation of blood flow is critical for neuronal function and survival. As discussed previously, perfusion of cerebral capillaries is primarily influenced by the diameter of the proximal parenchymal arteriole. In turn, the upstream penetrating arteriole and pial arteries must also dilate or constrict to optimize blood flow and perfusion pressure across the whole network. The cerebral circulation uses mechanisms of extrinsic and intrinsic innervation, along with metabolic, myogenic, and neurogenic autoregulation to ensure that blood flow is tightly regulated throughout the entire brain $[2,108,116]$. Autoregulation in particular is critical for maintaining cerebral perfusion pressure (CPP) within a physiological zone- if CPP drops too low $(<50 \mathrm{mmHg})$, neurons will quickly become ischemic; if CPP becomes too high (>160mmHg), BBB integrity may become compromised, elevated hydrostatic pressure may hamper diffusion and ion exchange, and cerebral edema can develop [2, 108, 116]. It is therefore critical that regulatory mechanisms integrate across all levels of circulation to ensure that perfusion is maintained and able to respond to specific increases in metabolic demand. 
The networks of pial arteries across the surface of the cortex are extrinsically innervated by perivascular nerve terminals. The density of these nerve bundles is higher in the larger, proximal pial arteries, and decreases in density as the pial arteries narrow in diameter; perivascular nervous synapses are almost completely absent at the point where penetrating arterioles dive down into the cortex $[2,116,119]$. Sympathetic innervation by these nerves on pial arteries with NE or 5-HT can cause acute vasoconstriction via $\alpha 1$-adrenoceptors and 5HT1B receptors, respectively. During conditions of health/normal blood flow, such induced constriction beyond basal vessel tone is not necessary, and therefor plays little to no role in regulating resting cerebral blood flow. However, sympathetic innervation becomes a fail-safe mechanism against chronic hypertension, shifting the myogenic tone curve upward to protect against edema and prevent so-called autoregulatory breakthrough [108, 127]. Parasympathetic innervation of pial arteries results in vasodilation through neurotransmitters such as NO, from neuronal NO synthase (nNOS), and ACh. These pathways help increase bulk blood flow during periods of heightened awareness, and also to help compensate/increase collateral flow during cerebral ischemia [2, 109, 118].

Penetrating and parenchymal arterioles lack the nerve bundles found in pial arteries are therefore not affected by extrinsic stimuli in the same manner as pial arteries. The $\alpha 1$ adrenoceptors and 5-HT1B receptors are absent, replaced by $\beta 1$ adrenoceptors and other 5-HT receptor subtypes $[108,113,119]$. Instead of constricting to NE and 5-HT as pial arteries do, the smaller arterioles will actually dilate in the presence of these neurotransmitters. Penetrating and parenchymal arterioles are instead more heavily innervated by intrinsic stimuli coming from the surrounding astrocytes, glial cells, and interneurons in their immediate vicinity [2, 128, 129]. Release of GABA, nNOS - produced NO, ACh, and even 5-HT can each induce vasodilation in 
penetrating and parenchymal arterioles, and brain slice culture studies have shown that simply increasing neuronal firing by electrical stimulation was sufficient to induce vasodilation through a calcium mediated pathway $[2,113,119,129]$.

Autoregulation through myogenic tone and myogenic reactivity are important mechanisms of blood flow control at all levels of the cerebrovasculature. Cerebral myogenic tone allows cerebral arteries to dilate or constrict to maintain constant perfusion at different levels of blood pressure and maintain a constant vessel luminal diameter at basal blood pressure [2]. Myogenic reactivity allows the vessels to respond to acute changes in cerebral perfusion pressure and blood flow; increases in perfusion pressure are sensed by stretch receptors in the vascular endothelium and vascular smooth muscle, which then stimulate vasoconstriction to maintain constant blood flow $[2,129]$. Decreases in perfusion pressure, in turn, activate pathways that cause vasodilation. At the pial level, myogenic autoregulation is able to adjust blood flow appropriately across a wide range of pressures [130], estimated generally to be $\sim 50 \mathrm{mmHg}$ to $\sim 160 \mathrm{mmHg}$. Myogenic autoregulation is perhaps strongest in the penetrating and parenchymal arterioles $[2,117,128,131-133]$, and helps to closely regulate perfusion in lieu of intrinsic or metabolic signaling for increased blood flow. CPP beyond this physiologic range, however, can cause vessels to lose their myogenic tone and become passive, resulting in dangerously high levels of cerebral perfusion $[2,117]$.

Autoregulation based on metabolic demand is another significant factor in controlling cerebral blood flow. This mechanism, also commonly referred to as flow-metabolism coupling or functional hyperemia, utilizes increases in $\mathrm{H}^{+}, \mathrm{K}^{+}$, and adenosine that occur as a result of increased neuronal firing/activation to induce vasodilation $[2,107,113,134,135]$. Adenosine levels rise as a result of increased neuronal activity, with evidence linking glutamate signaling to 
adenosine release [136]. $\mathrm{H}^{+}$and $\mathrm{K}^{+}$are also known byproducts of synaptic activity in neurons $[128,134,135,137]$. Increases in each of these molecules are therefore reflective of neuronal activity, and blood flow increases to provide the necessary increases in $\mathrm{O}_{2}$ and glucose. Acute hypoxia, which causes a reduction in oxidative respiration and a decrease in ATP levels, causes $\mathrm{K}_{\mathrm{ATP}}$ channels to activate in vascular smooth muscle $[123,138]$. Opening of $\mathrm{K}_{\mathrm{ATP}}$ channels allows an efflux of $\mathrm{K}^{+}$, resulting in hyperpolarization of the smooth muscle cells and causing vasodilation of the vessel. Hypoxia has also been shown to stimulate NO production within the endothelium, further promoting vasodilation. Similarly, increases in circulating $\mathrm{H}^{+}$as a result of hypercapnia will also cause vasodilation of cerebral vessels $[2,108]$. While NO-mediated vasodilation in response to shear stress can temporarily overcome myogenic constriction, autoregulation by myogenic forces is stronger and will restore vessel diameter to baseline [2, 117].

Lastly, the cerebral vascular endothelium produces several vasoactive substances that help regulate cerebral blood flow. Because of its immediate proximity to the blood, circulating factors, endocrine signaling molecules, and leukocyte-derived signaling molecules each can act through the endothelium to dilate or constrict vessels $[2,108,114,135]$. The most potent vasodilatory mechanism is that of nitric oxide (NO), produced by endothelial NO synthase (eNOS) [108, 109, 139]. Cerebral eNOS oxidizes 1-Arginine into l-Citrulline and NO, and has repeatedly been shown to be an important mediator of vasodilation in pial arteries, penetrating arterioles, and parenchymal arterioles $[2,24,36,45,107,108,140]$. The endothelium also enables vasodilation through the production of endothelium-derived hyperpolarizing factors (EDHF), such as the COX metabolites prostacyclin and prostaglandin E2, and hydrogen peroxide $[24,115,116]$. Each of these signaling pathways induces hyperpolarization of the vascular 
smooth muscle, resulting in relaxation of the muscle and dilation of the blood vessel. Cerebral endothelial cells also synthesize and release endothelin-1, a potent vasoconstrictor by biding the $\mathrm{ET}_{\mathrm{A}}$ receptor on cerebral vascular smooth muscle cells $[2,108,141]$. Endothelin-1, in conditions of health, affects remarkably long periods of constriction, and contributes to maintenance of vascular tone. However, in conditions of vascular dysfunction or cerebral hemorrhage, increased release of endothelin-1 can lead to hypertension and cerebral vasospasm [108].

In sum, the cerebrovasculature is responsible for the regulation and delivery of consistent blood flow to the entire brain. Blood flow is tightly regulated by a system of extrinsic, intrinsic, autoregulatory, metabolic, and endothelium-derived factors, which under conditions of health work together to ensure constant, uninterrupted blood flow throughout the brain, and increases flow to areas of increased neuronal activity.

\section{Part 4: Mechanisms of Cerebrovascular Disease- Metabolic Syndrome, Ischemic Stroke,}

\section{Vascular Cognitive Impairments}

The multitude of regulatory and autoregulatory mechanisms regulating healthy cerebral blood flow provides numerous potential points for pathological disruption. Indeed, each category of cerebrovascular disease is characterized by distinct pathophysiological deviations that impact the function of the vascular endothelium, vascular smooth muscle, and mechanics of the cerebral vessels. To properly contextualize the role each of these pathways play in affecting cerebrovascular disease progression, it is important to first characterize the cerebrovascular dysfunction and remodeling that is inherent to the constituent pathologies underlying metabolic syndrome, as these mechanisms are the driving factors that place patients at risk of cerebrovascular disease in the first place. 
Pre-clinical investigations of vascular function in metabolic syndrome often utilize rodent models of this disease, including the obese Zucker rat (OZR), Zucker diabetic fatty (ZDF), and Goto-Kakizaki (GK) rat [142-144]. OZR and ZDF, which possess a truncated leptin receptor, both are hyperphagic and rapidly develop moderate hypertension, hyperglycemia, elevated lipids and cholesterol, and insulin insensitively as a consequence of overeating [142, 143]. GK is a non-obese model of type 2 diabetes, which derives from genetic mutations affecting pancreatic $\mathrm{B}$ cells and insulin receptors in peripheral tissues [144]. While the etiology of metabolic syndrome in Zucker-derived animals more closely mirrors the human condition, all three of these models have been used to make translationally-relevant observations of vascular dysfunction [41-43, $121,122,145,146]$. Consequently, these rats, and related leptin receptor modified mice, are also popularly used to study ischemic stroke and vasculopathy-associated cognitive decline.

\section{Mechanisms of Cerebrovascular Dysfunction}

While rodent models of diabetes had been popular models for studying diabetesassociated vascular dysfunction in the peripheral circulation, Mayhan's 1989 paper was the first to denote a specific impairment of dilation in pial arteries of diabetic rats [147]. Despite utilization of a type-1 diabetic model, he reported an endothelial etiology for cerebrovascular dysfunction that would serve as a basis for investigation in other models. Building on this work, Schwaninger et al. found that pial arteries in OZR demonstrated impaired vasodilation to ACh and adenosine, but normal dilation to nitroglycerin [146]. They also reported elevated protein expression of eNOS in pooled pial arteries of OZR; together, their results suggested that a mechanism specifically impairing endothelium-dependent NO production was causing the vascular dysfunction. Erdos et al. reported that dilation to ACh and PGI2 was impaired in basilar arteries of OZR, but not to an exogenous NO donor [148]. They then treated the basilar artery 
with SOD and observed total recovery of vasodilation, indicating that ROS, specifically superoxide, was limiting bioavailability of endothelial NO. Phillips et al. confirmed vascular dysfunction in ex vivo MCA from OZR, and further reported increased oxidative stress, increased sensitivity to sympathetic vasoconstrictors, and elevated basal vascular tone [149]. However, Phillips et al reported only partial restoration of dilator activity after treatment with TEMPOL, a potent ROS scavenger, and that TEMPOL treatment did not ameliorate increased myogenic reactivity or hyperconstriction to 5-HT. Additionally, they reported that no significant changes in vessel morphology or distensibility had developed, in stark contrast with skeletal muscle arterioles from OZR [149]. Overall, a significant body of research supports that acute scavenging of ROS by SOD, TEMPOL, or other antioxidants results in improved NO bioavailability and vasodilation, both in the peripheral and cerebral circulation [147-152].

Many types of ROS- superoxide, hydroxyl radicals, and peroxides- as well as reactive nitrogen species such as peroxynitrite have been implicated within the cerebral vasculature [23, $114,152,153]$. Several hypotheses have been tested to elucidate the source of ROS in the cerebrovasculature, as well as to investigate potential treatments for restoring cerebrovascular reactivity in models of diabetes and metabolic syndrome. One theory is that angiotensin II, through its binding of angiotensin type I (AT1) receptors and activation of NAD(P)H oxidase, increases generation of superoxide in the cerebrovasculature [113, 153-155]. Studies showing ROS-associated dysfunction of endothelial-dependent dilatory responses in aged mice [153, 154], renin-angiotensin system (RAS) activation and endothelial dysfunction in LPS treated mice [156], and improvement of vasodilation in losartan-treated type 1 diabetic rats [157] and enalapril-treated type 1 diabetic rats [158] all support this theory. RAS is upregulated in both 
hypertension and diabetes, and RAS inhibitors have been shown to significantly lower long-term risks of vascular complications in metabolic syndrome patients [159].

Figure 5: Reactive Oxygen Species Impair Nitric Oxide Bioavailability in the Cerebrovascular Endothelium and Induce Vascular Dysfunction

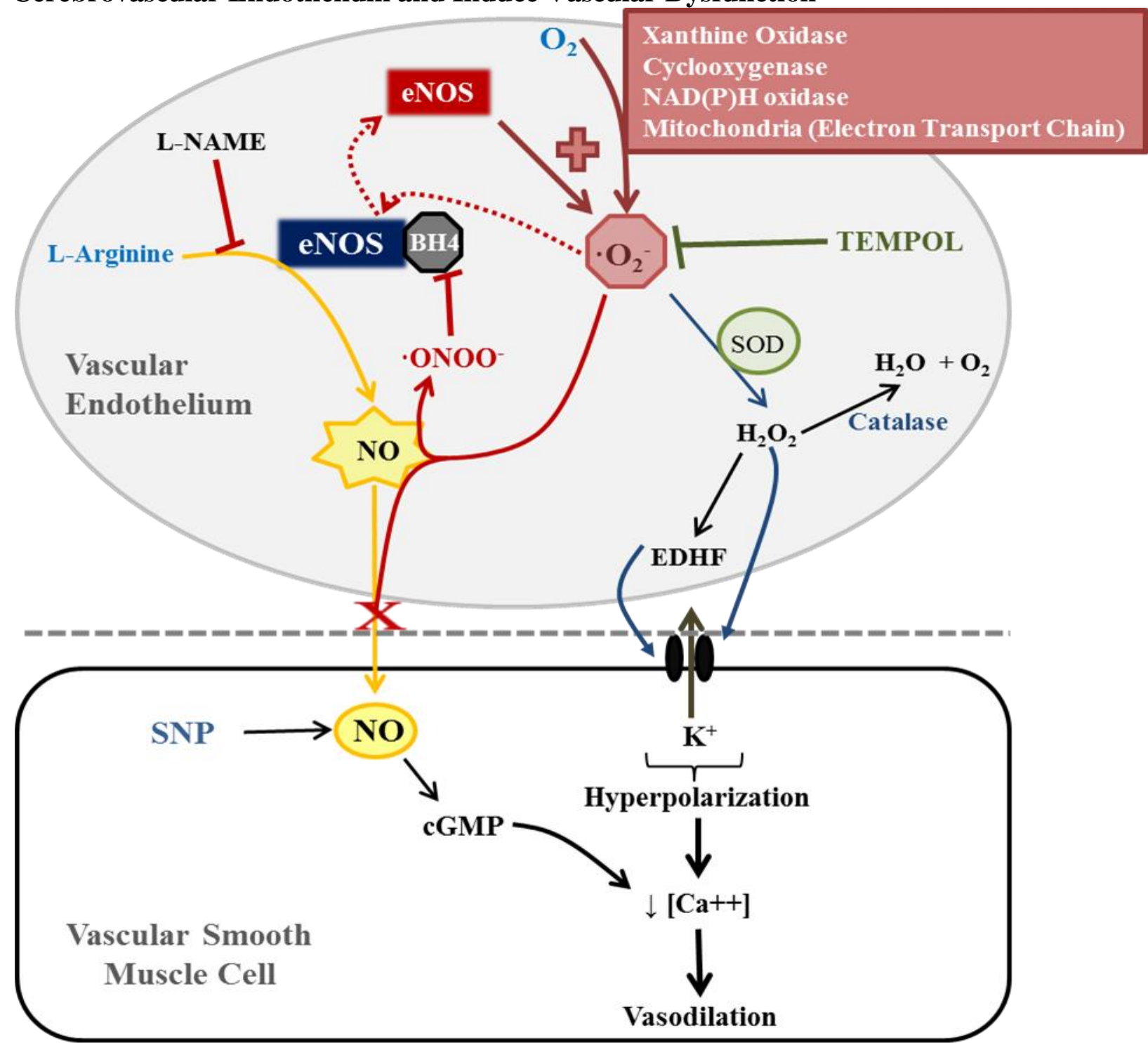

Cerebral production of superoxide is generated from several sources, including $\mathrm{NAD}(\mathrm{P}) \mathrm{H}$ oxidases, especially isoforms Nox2 and Nox4 [160, 161], endothelial and smooth muscle mitochondria [162], xanthine oxidase, uncoupled eNOS, and other less common oxygenase enzymes $[23,107,150,152,163]$. Superoxide $\left(\mathrm{O}_{2} \cdot\right)$ especially has been effectively targeted in 
pre-clinical models, improving NO•-mediated responses in several models, including: elderly animals $[153,154,164]$, hypertensive $[165,166]$, hypercholesterolaemic $[167,168]$, diabetic $[149,157,169]$, and stroke patents $[114,170,171]$. Use of apocynin, a broad inhibitor of $\mathrm{NAD}(\mathrm{P}) \mathrm{H}$ oxidases, was able to restore reactivity of pial vessels in diabetic rats $[152,155,166]$, while another study found that Rosuvastatin treatment improved dilator reactivity of basilar arteries in obese Zucker rats by reducing $\mathrm{NAD}(\mathrm{P}) \mathrm{H}$-produced $\mathrm{O}_{2} \cdot[152,166]$. While $\mathrm{NAD}(\mathrm{P}) \mathrm{H}$ oxidases are widely accepted as a major source of cerebral ROS [169, 172], some debate remains as to whether Nox 2 or Nox4 is the primary source of NAD(P)H oxidase generated ROS [161, $164,165,168,173]$ Nox 4 is highly expressed in endothelial cells of cerebral arteries in rats [160, 173-175], while Nox2 is highly expressed in mice MCAs as well as in circulating leukocytes $[107,161,164,168,174,176]$. Nox2 has been linked to cerebrovascular dysfunction in dietinduced obese mice $[161,165,166,177]$, while Nox4 was recently shown to increase ROS production in cerebral endothelial cells in response to TNF- $\alpha$ [173]. Elevated levels of ROS can also lead to endothelial cell mitochondrial dysfunction, and induce further overproduction of ROS within the mitochondria $[155,178]$. Metabolic syndrome has also itself been associated with increased mitochondrial production of $\operatorname{ROS}[162,169,179]$. Lastly, ROS can lead to uncoupling of eNOS from its cofactor BH4 [180]. Uncoupled eNOS will reduce NO production, despite continued high protein expression of eNOS, and instead generate superoxide.

Chronic inflammation is also strongly linked to vascular dysfunction. TNF- $\alpha$, an inflammatory cytokine strongly linked to obesity, metabolic syndrome, depression, and many other chronic disease states, stimulates production of NF-kB, a critical mediator of inflammatory responses, apoptosis, and expression of growth factors. NF-kB stimulates pro-inflammatory cytokines and adhesion molecules, such as MCP-1 [181] and ICAM-1 [182], and is implicated in 
endothelial dysfunction as well as arterial stiffening and plaque formation. While plaque formation is uncommon in pial and parenchymal arterioles, TNF- $\alpha$ has been linked to activation of Nox4 and subsequent generation of ROS [173]. IL-6, another inflammatory mediator associated with metabolic syndrome, is the primary regulator of C-reactive protein (CRP); increased CRP infiltration of the vasculature, results in inhibition of eNOS and angiotensinstimulated ROS production [183]. IL-6 has also been linked to mediating the endothelial effects of angiotensin II [165]. Studies in the peripheral circulation have shown that reducing inflammation with statins can improve NO bioavailability [184, 185], and a study in the cerebral circulation found that Rosuvastatin can reduce cerebral ROS via mechanisms independent of lipid-lowering effects [150]. The intersection between inflammatory mediators and ROS production have clear implications for endothelial dysfunction in the cerebral circulation and presents an array of potential targets for developing therapeutics to improve cerebral blood flow.

In humans, type 2 diabetes has been linked to impaired reactivity of MCAs to hypercapnia, as assessed by trans-cranial Doppler ultrasound. This effect was linked to increased arterial stiffening [186] and endothelial dysfunction associated with hyperglycemia. Building on studies that demonstrated increased basal cerebral blood flow in healthy older adults, Wong et al have shown that resveratrol supplementation was efficient at improving flow-mediated dilation in brachial arteries of diabetic patients [187], and were further able to demonstrate a similar improvement in MCA vasodilator responsiveness to a hypercapnic stimulus [187]; they then demonstrated improvements in flow-mediated dilation in obese adults, and hypertensive obese adults [188, 189]. Resveratrol, a polyphenol molecule which upregulates SOD2 in vitro, is a controversial antioxidant [190], but these results are in accordance with findings that antioxidant therapies consistently improve vascular reactivity in models of metabolic syndrome. 
Metabolic syndrome and diabetes are also linked to structural and mechanical changes in the cerebrovasculature. In diabetic rats, the basement membrane of cerebral microvessels is thickened, and swelling occurs in the pericytes and astrocytic end feed [120,121, 126, 191]. Increased collagen can build up in the vessel wall, and degeneration of endothelial cells may also develop[45]. Additionally, the BBB may become more permeable, leading to risk of edema and transport of pathogens or harmful molecules into the cerebrum. Several studies have reported an increase in myogenic tone in both T1D [128] and T2D [171, 192] rat models. In the GK rat, increased myogenic tone was associated with elevated levels of peroxynitrite, and animals exhibited impaired functional hyperemia and reduced regulation of CBF $[121,171]$.

While diabetes/metabolic syndrome are associated with rarefaction and decreased angiogenesis in the periphery $[42,184,193,194]$, a recent slate of studies from Dr. Fagan's laboratory reported neovascularization and angiogenesis increasing vessel density in the cortex of non-obese, type 2 diabetic GK rats [120-122, 145]. In contrast, Beauquis et al reported a reduction in microvessel density in the hippocampus of the same rat model [195]. Interestingly, the Fagan studies reported that the new vessels were often tortuous, non-viable, and nonperfused, suggesting that a functional hypo-perfusion could result. A thickening of cerebral vessel walls also is known to occur in hypertension [196, 197], as is rarefaction of human peripheral microvessels [198] and rodent cerebral cortical microvessels [124]. Work from Munzenmaier and Green supports that blockage of AT1 receptor increases cortical microvessel density concomitant to blood pressure changes, but could also be due to reduction of AT1associated ROS production [124]. Clearly, further study is needed to interrogate angiogenesis/rarefaction in the cerebral circulation and what impacts this may hold for cerebrovascular disease. 


\section{Metabolic Syndrome, Diabetes, and Ischemic Stroke}

As detailed above, rodent models of metabolic syndrome, diabetes, and hypertension all develop pathologies of cerebrovascular vascular function and structure, with obvious implications for outcomes of ischemic stroke. Indeed, ischemic stroke studies in diabetic mice report increased infarct size, edema, and risk of hemorrhagic transformation. Pre-treatment of these mice with peroxisome proliferator-activated receptor (PPAR)- $\gamma$ agonists darglitazone, rosiglitazone, and pioglitazone is neuroprotective in stroke, leading to smaller infarcts and better functional outcomes $[45,126]$. These studies support that acute usage of (PPAR)- $\gamma$ agonists may have clinical utility, especially in hyperglycemic patients.

The literature is somewhat more conflicting in rats: in the GK rat, ischemic stroke/reperfusion resulted in smaller infarcts, but increased incidence of hemorrhage and increased neurological deficits, as the infarction was more localized to the striatum, compared to the cortex in controls $[45,121,122,145,171,191]$. In OZR, stroke caused significantly larger infarcts and MCA remodeling compared to control, which could be mitigated by chronically controlling blood pressure prior to the stroke $[199,200]$. One potential explanation may be that neovascularization and angiogenesis is increased in the cortex of GK, but not in deeper brain regions; therefore, the newer, underdeveloped vessels can perfuse the cortex better during stroke, but are more prone to rupture and hemorrhage, while deeper brain regions are under-perfused, leading to infarcts and more severe neurological deficits. If the OZR has reduced vessel density in both the cortex and striatum, then larger infarcts and increased impairment would be the expected outcome. Additionally, GK is non-obese and non-hypetesnive, both of which are risk factors for rarefaction. A recent study by Abdelsaid et al found that peroxynitrite production is 
elevated following ischemia-reperfusion in GK rats, and that treatment with metformin immediately after stroke can reduce nitrative-stress related damages and promote angiogenesis in infarcted regions of the brain in this model [145], suggesting it may be a hyperglycemia-specific pathology.

Ischemic stroke and reperfusion have a litany of pathophysiological effects on pial artery, penetrating arteriole, and parenchymal arteriole function, which are further confounded by preexisting cerebrovascular dysfunction. During stroke and reperfusion, there is a sharp increase in circulating superoxide and peroxynitrite, which can negatively impact myogenic tone in both large and small arteries, as well as reduce NO bioavailability in the cerebral endothelium [36, 37 , $170,172,200]$. Endothelial NO is an important mediator of the protective response to stroke, as NO improves dilation of conduits and collaterals, as well as inhibits platelet aggregation and leukocyte adhesion. Reduced NO bioavailability therefore reduces collateral flow, and leads to increased risk of further blockages in cerebral arterioles by small clots and platelet plugs [2, 117, 201, 202]. In metabolic syndrome, NO bioavailability is already reduced, and levels of oxidative stress are elevated; further ROS and RNS production may lead to a near-total loss of NO bioavailability during ischemia-reperfusion, severely impairing collateral flow and increasing infarct size through poor perfusion and further aggregation of platelets [41, 42, 149, 193]. While hypoxia can lead to dilation of MCAs and other conduits, this response is impaired in metabolic syndrome [203-205], and does not appear to be present in parenchymal vessels. Dilation of parenchymal vessels to $\mathrm{NO}, \mathrm{EDHF}$, or other hyperpolarizing factors is therefore critical during stroke, yet these responses are already impaired in many stroke comorbidities. Many of the pathological effects of ischemic stroke are a result of the inflammatory response and oxidative stress that develops during ischemia/reperfusion [202]. These same mechanisms are already 
dysfunctional in diabetes and metabolic syndrome, which are both prevalent risk factors for ischemic stroke. Further research is certainly needed to investigate how these diseases affect stroke outcomes, and consideration of their complications towards of any new stroke therapy is of critical importance.

\section{Metabolic Syndrome, Vascular Dysfunction, and Neurologic Function}

A recent study reported that the onset of MetSyn in OZR was associated with cognitive impairments in memory testing, likely through hippocampal-dependent pathways [206]. Similar impairments in cognitive function and memory recall have been observed in other rat strains fed high-fat Western diets [207-209]. Rats which underwent mild disruption of cerebral blood flow through a transient ischemia model developed cognitive decline and memory impairments [210], and rats exposed to elevated oxidative stress developed cognitive deficits with direct implications for health of the hippocampus and frontal cortex [211]. Viewed as a whole, these studies provide evidence that the vascular pathologies inherent to MetSyn (and other types CVD) contribute directly to cognitive decline. Similarly, cognitive impairments in aged rats have been directly linked to regional changes in brain glucose availability and metabolism [212]. With a growing body of clinical and epidemiological literature implicating MetSyn as a contributing factor towards cognitive impairment [27], and high-fat diets with cognitive decline later in life [213] and cognitive changes in older adults [214], the OZR-specific structural and physiological vascular impairments that are highlighted in this study may provide a novel pathway for preventing or treating cognitive impairments in at-risk adults. 
Figure 6: Inflammation and ROS from Metabolic Syndrome and Depression Impairs Cerebrovascular Blood Flow with Implications for Ischemic Stroke

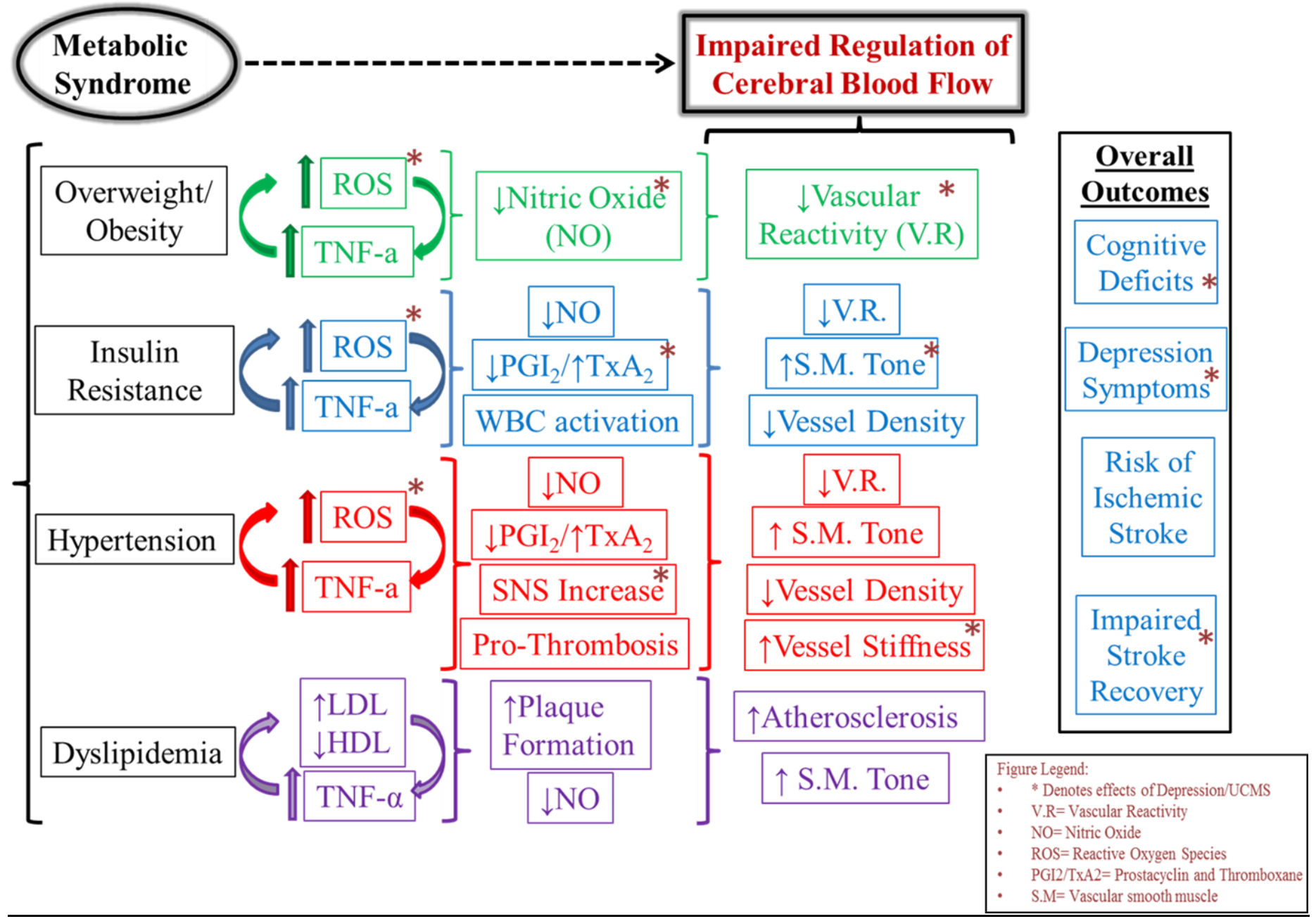

\section{Part 5: Summary and statement of aims for writing the dissertation}

As summarized above, endothelial vascular dysfunction is known to play a critical in the pathophysiology of many disease states including hypertension, diabetes, dyslipidemia, and metabolic syndrome. When coupled with evidence of cerebral rarefaction and/or neovascularization of non-viable vessels, such deficits in regulating cerebral perfusion have clear implications for outcomes in ischemic stroke, and in the brain's long-term ability to match blood supply with increased demand by metabolically active regions of the brain. The literature thus far 
represents a diversity of rodent models, but two themes remain clear: oxidative stress impairs vasodilation and vascular reactivity in the cerebral circulation, and cerebral perfusion and disease outcome are significantly linked.

By utilizing a highly translational rodent model of metabolic syndrome- the obese Zucker rat- this dissertation aims to more fully interrogate the mechanisms through which metabolic syndrome, and its constituent pathologies, contribute to cerebrovascular dysfunction and perfusion impairments. This model will then be used to study how environmental perturbations effect alterations in cerebrovascular function: the effects of chronic stress and depression, a common comorbidity of metabolic syndrome, are evaluated to further define the relationship between depression and vascular disease, and an exercise training regime is implemented to investigate how the anti-oxidant/pro-angiogenic effects of exercise impact cerebrovascular dysfunction from stress/depression in the OZR. Lastly, OZR is presented as a novel model for studying vascular dysfunction and oxidative stress during ischemic stroke. 


\section{LITERATURE CITED}

1. National Health Service. Cerebrovascular Disease. 2015 [cited 20165 May]; Available from: http://www.nhs.uk/conditions/cerebrovascular-disease/pages/definition.aspx

2. Cipolla, M.J., in The Cerebral Circulation. 2009: San Rafael (CA).

3. Good, D.C., Cerebrovascular Disease, in Clinical Methods: The History, Physical, and Laboratory Examinations, H.K. Walker, W.D. Hall, and J.W. Hurst, Editors. 1990: Boston.

4. Banasiak, K.J., Y. Xia, and G.G. Haddad, Mechanisms underlying hypoxia-induced neuronal apoptosis. Prog Neurobiol, 2000. 62(3): p. 215-49.

5. Bickler, P.E. and P.H. Donohoe, Adaptive responses of vertebrate neurons to hypoxia. J Exp Biol, 2002. 205(Pt 23): p. 3579-86.

6. Frizzell, J.P., Acute stroke: pathophysiology, diagnosis, and treatment. AACN Clin Issues, 2005. 16(4): p. 421-40; quiz 597-8.

7. Alhusban, A., et al., Artery reopening is required for the neurorestorative effects of angiotensin modulation after experimental stroke. Exp Transl Stroke Med, 2016. 8: p. 4.

8. Prevention, C.f.D.C.a. Stroke. 2016 [cited 20164 April]; Available from:

http://www.cdc.gov/stroke/index.htm.

9. Ankarcrona, M., et al., Glutamate-Induced Neuronal Death - a Succession of Necrosis or Apoptosis Depending on Mitochondrial-Function. Neuron, 1995. 15(4): p. 961-973.

10. Carmody, R.J., A.J. McGowan, and T.G. Cotter, Reactive oxygen species as mediators of photoreceptor apoptosis in vitro. Experimental Cell Research, 1999. 248(2): p. 520-530.

11. Deb, P., S. Sharma, and K.M. Hassan, Pathophysiologic mechanisms of acute ischemic stroke: An overview with emphasis on therapeutic significance beyond thrombolysis.

Pathophysiology, 2010. 17(3): p. 197-218.

12. American Stroke Association. Stroke Treatments. 2013 [cited 20165 May]; Available from: http://www.strokeassociation.org/STROKEORG/AboutStroke/Treatment/StrokeTreatments_UCM_310892_Article.jsp\#.V0VUMOT3Ohm.

13. Easton, J.D., et al., Definition and evaluation of transient ischemic attack: a scientific statement for healthcare professionals from the American Heart Association/American Stroke Association Stroke Council; Council on Cardiovascular Surgery and Anesthesia; Council on Cardiovascular Radiology and Intervention; Council on Cardiovascular Nursing; and the Interdisciplinary Council on Peripheral Vascular Disease. The American Academy of Neurology 
affirms the value of this statement as an educational tool for neurologists. Stroke, 2009. 40(6): p. 2276-93.

14. Prabhakaran, S., I. Ruff, and R.A. Bernstein, Acute stroke intervention: a systematic review. JAMA, 2015. 313(14): p. 1451-62.

15. Romano, J.G. and R.L. Sacco, Decade in review-stroke: progress in acute ischaemic stroke treatment and prevention. Nat Rev Neurol, 2015. 11(11): p. 619-21.

16. Hebert, R. and C. Brayne, Epidemiology of vascular dementia. Neuroepidemiology, 1995. 14(5): p. 240-57.

17. Roberts, R. and D.S. Knopman, Classification and epidemiology of MCI. Clin Geriatr Med, 2013. 29(4): p. 753-72.

18. Roth, G.A., et al., Methods for Estimating the Global Burden of Cerebrovascular Diseases. Neuroepidemiology, 2015. 45(3): p. 146-51.

19. Chin, J.H. and N. Vora, The global burden of neurologic diseases. Neurology, 2014. 83(4): p. 349-51.

20. Hebert, R., et al., Vascular dementia : incidence and risk factors in the Canadian study of health and aging. Stroke, 2000. 31(7): p. 1487-93.

21. Breitner, J.C., Mild cognitive impairment and progression to dementia: new findings. Neurology, 2014. 82(4): p. e34-5.

22. Parnetti, L., et al., Pathogenetic mechanisms in vascular dementia. Int J Clin Lab Res, 1994. 24(1): p. 15-22.

23. Chrissobolis, S. and F.M. Faraci, The role of oxidative stress and NADPH oxidase in cerebrovascular disease. Trends Mol Med, 2008. 14(11): p. 495-502.

24. Faraci, F.M., Protecting against vascular disease in brain. Am J Physiol Heart Circ Physiol, 2011. 300(5): p. H1566-82.

25. Aguilar, M., et al., Prevalence of the metabolic syndrome in the United States, 2003-2012. JAMA, 2015. 313(19): p. 1973-4.

26. American Heart Association. Metabolic Syndrome. 2014 [cited 20163 March]; Available from: http://www.heart.org/HEARTORG/Conditions/More/MetabolicSyndrome/MetabolicSyndrome_UCM_002080_SubHomePage.jsp.

27. Panza, F., et al., Metabolic syndrome and cognitive impairment: current epidemiology and possible underlying mechanisms. J Alzheimers Dis, 2010. 21(3): p. 691-724. 
28. Roberts, R.O., et al., Metabolic syndrome, inflammation, and nonamnestic mild cognitive impairment in older persons: a population-based study. Alzheimer Dis Assoc Disord, 2010. 24(1): p. 11-8.

29. Solfrizzi, V., et al., Metabolic syndrome and the risk of vascular dementia: the Italian Longitudinal Study on Ageing. J Neurol Neurosurg Psychiatry, 2010. 81(4): p. 433-40.

30. Writing Group, M., et al., Heart Disease and Stroke Statistics-2016 Update: A Report From the American Heart Association. Circulation, 2016. 133(4): p. e38-60.

31. Seshadri, S., et al., The lifetime risk of stroke: estimates from the Framingham Study. Stroke, 2006. 37(2): p. 345-50.

32. Chapman, S.N., et al., Current perspectives on the use of intravenous recombinant tissue plasminogen activator (tPA) for treatment of acute ischemic stroke. Vasc Health Risk Manag, 2014. 10: p. 75-87.

33. Hafez, S., et al., Hyperglycemia, acute ischemic stroke, and thrombolytic therapy. Transl Stroke Res, 2014. 5(4): p. 442-53.

34. Schwamm, L.H., et al., Temporal trends in patient characteristics and treatment with intravenous thrombolysis among acute ischemic stroke patients at Get With The GuidelinesStroke hospitals. Circ Cardiovasc Qual Outcomes, 2013. 6(5): p. 543-9.

35. Dawson, J. and M. Walters, New and emerging treatments for stroke. Br Med Bull, 2006. 7778: p. 87-102.

36. Palomares, S.M. and M.J. Cipolla, Vascular Protection Following Cerebral Ischemia and Reperfusion. J Neurol Neurophysiol, 2011. 2011.

37. Chamorro, A., et al., Neuroprotection in acute stroke: targeting excitotoxicity, oxidative and nitrosative stress, and inflammation. Lancet Neurol, 2016.

38. Brown, W.V., Metabolic syndrome and risk of stroke. Clin Cornerstone, 2004. 6 Suppl 3: p. S30-4.

39. Boden-Albala, B., et al., Metabolic syndrome and ischemic stroke risk: Northern Manhattan Study. Stroke, 2008. 39(1): p. 30-5.

40. Tyndall, A.V., et al., Cardiometabolic risk factors predict cerebrovascular health in older adults: results from the Brain in Motion study. Physiol Rep, 2016. 4(8).

41. Chantler, P.D. and J.C. Frisbee, Arterial function in cardio-metabolic diseases: from the microcirculation to the large conduits. Prog Cardiovasc Dis, 2015. 57(5): p. 489-96. 
42. Frisbee, J.C., Reduced nitric oxide bioavailability contributes to skeletal muscle microvessel rarefaction in the metabolic syndrome. Am J Physiol Regul Integr Comp Physiol, 2005. 289(2): p. R307-R316.

43. Frisbee, J.C. and M.D. Delp, Vascular function in the metabolic syndrome and the effects on skeletal muscle perfusion: lessons from the obese Zucker rat. Essays Biochem, 2006. 42: p. 14561.

44. Frisbee, J.C., et al., Integration of skeletal muscle resistance arteriolar reactivity for perfusion responses in the metabolic syndrome. Am J Physiol Regul Integr Comp Physiol, 2009. 296(6): p. R1771-82.

45. Ergul, A., et al., Cerebrovascular complications of diabetes: focus on stroke. Endocr Metab Immune Disord Drug Targets, 2012. 12(2): p. 148-58.

46. Furukawa, S., et al., Increased oxidative stress in obesity and its impact on metabolic syndrome. J Clin Invest, 2004. 114(12): p. 1752-61.

47. Prevention, C.f.D.C.a. Alzheimer's Disease. 2016 [cited 20165 May]; Available from: http://www.cdc.gov/aging/aginginfo/alzheimers.htm.

48. Seshadri, S. and P.A. Wolf, Lifetime risk of stroke and dementia: current concepts, and estimates from the Framingham Study. Lancet Neurol, 2007. 6(12): p. 1106-14.

49. Seshadri, S., et al., Lifetime risk of dementia and Alzheimer's disease. The impact of mortality on risk estimates in the Framingham Study. Neurology, 1997. 49(6): p. 1498-504.

50. Alzheimer's Association. 2016 Alzheimer's Disease Facts and Figures. 2016 [cited 20164 April]; Available from: http://www.alz.org/facts/.

51. CDC.gov Prevalence of Stroke- United States, 2006-2010. 2012. 379-382.

52. Ervin, R.B., Prevalence of metabolic syndrome among adults 20 years of age and over, by sex, age, race and ethnicity, and body mass index: United States, 2003-2006. Natl Health Stat Report, 2009(13): p. 1-7.

53. Kessler, R.C., et al., The epidemiology of major depressive disorder: results from the National Comorbidity Survey Replication (NCS-R). JAMA, 2003. 289(23): p. 3095-105.

54. Kessler, R.C., et al., Sex and depression in the National Comorbidity Survey. I: Lifetime prevalence, chronicity and recurrence. J Affect Disord, 1993. 29(2-3): p. 85-96.

55. Penninx, B.W., et al., Depression and cardiac mortality: results from a community-based longitudinal study. Arch Gen Psychiatry, 2001. 58(3): p. 221-7. 
56. Whiteford, H.A., et al., Global burden of disease attributable to mental and substance use disorders: findings from the Global Burden of Disease Study 2010. Lancet, 2013. 382(9904): p. 1575-86.

57. Kendler, K.S., et al., A Swedish national twin study of lifetime major depression. Am J Psychiatry, 2006. 163(1): p. 109-14.

58. Kessler, R.C., et al., Lifetime prevalence and age-of-onset distributions of DSM-IV disorders in the National Comorbidity Survey Replication. Arch Gen Psychiatry, 2005. 62(6): p. 593-602. 59. Bayramgurler, D., et al., The effect of etanercept on aortic nitric oxide-dependent vasorelaxation in an unpredictable chronic, mild stress model of depression in rats. Eur J Pharmacol, 2013. 710(1-3): p. 67-72.

60. Chrapko, W.E., et al., Decreased platelet nitric oxide synthase activity and plasma nitric oxide metabolites in major depressive disorder. Biol Psychiatry, 2004. 56(2): p. 129-34. 61. d'Audiffret, A.C., et al., Depressive behavior and vascular dysfunction: a link between clinical depression and vascular disease? J Appl Physiol (1985), 2010. 108(5): p. 1041-51. 62. Isingrini, E., et al., Fluoxetine effect on aortic nitric oxide-dependent vasorelaxation in the unpredictable chronic mild stress model of depression in mice. Psychosom Med, 2012. 74(1): p. 63-72.

63. Isingrini, E., et al., Altered aortic vascular reactivity in the unpredictable chronic mild stress model of depression in mice: UCMS causes relaxation impairment to ACh. Physiol Behav, 2011. 103(5): p. 540-6.

64. Kamper, E.F., et al., Sex differences in oxidant/antioxidant balance under a chronic mild stress regime. Physiol Behav, 2009. 98(1-2): p. 215-22.

65. Plante, G.E., Depression and cardiovascular disease: a reciprocal relationship. Metabolism, 2005. 54(5 Suppl 1): p. 45-8.

66. Stanley, S.C., et al., Protective effect of sex on chronic stress- and depressive behaviorinduced vascular dysfunction in BALB/cJ mice. J Appl Physiol (1985), 2014. 117(9): p. 959-70. 67. Taylor, W.D., H.J. Aizenstein, and G.S. Alexopoulos, The vascular depression hypothesis: mechanisms linking vascular disease with depression. Mol Psychiatry, 2013. 18(9): p. 963-74. 68. Bornstein, S.R., et al., Approaching the shared biology of obesity and depression: the stress axis as the locus of gene-environment interactions. Mol Psychiatry, 2006. 11(10): p. 892-902. 
69. Dimopoulos, N., et al., Elevation of plasma concentration of adhesion molecules in late-life depression. Int J Geriatr Psychiatry, 2006. 21(10): p. 965-71.

70. Chen, H., et al., Relationship of depression, stress and endothelial function in stable angina patients. Physiol Behav, 2013. 118: p. 152-8.

71. Ippoliti, F., N. Canitano, and R. Businaro, Stress and obesity as risk factors in cardiovascular diseases: a neuroimmune perspective. J Neuroimmune Pharmacol, 2013. 8(1): p. 212-26.

72. Luppino, F.S., et al., Overweight, obesity, and depression: a systematic review and metaanalysis of longitudinal studies. Arch Gen Psychiatry, 2010. 67(3): p. 220-9.

73. Pratt, L.A.B., Debra J. Depression in the U.S. Household Population, 2009-2012. 2014 [cited 201615 April]; Available from: http://www.cdc.gov/nchs/data/databriefs/db172.htm. 74. CDC.gov, Current Depression Among Adults --- United States, 2006 and 2008. MMWR 2010. 59(38): p. 1229-1235.

75. Antonijevic, I.A., Depressive disorders -- is it time to endorse different pathophysiologies? Psychoneuroendocrinology, 2006. 31(1): p. 1-15.

76. Barden, N., Implication of the hypothalamic-pituitary-adrenal axis in the physiopathology of depression. J Psychiatry Neurosci, 2004. 29(3): p. 185-93.

77. Hart, J., Jr., et al., Neuroimaging of cognitive dysfunction and depression in aging retired National Football League players: a cross-sectional study. JAMA Neurol, 2013. 70(3): p. 32635.

78. Krishnan, V. and E.J. Nestler, Linking molecules to mood: new insight into the biology of depression. Am J Psychiatry, 2010. 167(11): p. 1305-20.

79. Lambert, G., et al., Reduced brain norepinephrine and dopamine release in treatmentrefractory depressive illness: evidence in support of the catecholamine hypothesis of mood disorders. Arch Gen Psychiatry, 2000. 57(8): p. 787-93.

80. Pizzi, C., et al., Autonomic nervous system, inflammation and preclinical carotid atherosclerosis in depressed subjects with coronary risk factors. Atherosclerosis, 2010. 212(1): p. 292-8.

81. Raison, C.L. and A.H. Miller, When not enough is too much: the role of insufficient glucocorticoid signaling in the pathophysiology of stress-related disorders. Am J Psychiatry, 2003. 160(9): p. 1554-65. 
82. Grippo, A.J., et al., Chronic mild stress induces behavioral and physiological changes, and may alter serotonin $1 A$ receptor function, in male and cycling female rats. Psychopharmacology (Berl), 2005. 179(4): p. 769-80.

83. Haroon, E., C.L. Raison, and A.H. Miller, Psychoneuroimmunology meets neuropsychopharmacology: translational implications of the impact of inflammation on behavior. Neuropsychopharmacology, 2012. 37(1): p. 137-62.

84. Maddock, C. and C.M. Pariante, How does stress affect you? An overview of stress, immunity, depression and disease. Epidemiol Psichiatr Soc, 2001. 10(3): p. 153-62.

85. Pariante, C.M., et al., Antidepressants enhance glucocorticoid receptor function in vitro by modulating the membrane steroid transporters. Br J Pharmacol, 2001. 134(6): p. 1335-43. 86. Pariante, C.M. and A.H. Miller, Glucocorticoid receptors in major depression: relevance to pathophysiology and treatment. Biol Psychiatry, 2001. 49(5): p. 391-404.

87. Sapolsky, R.M., Stress hormones: good and bad. Neurobiol Dis, 2000. 7(5): p. 540-2. 88. Burke, H.M., et al., Depression and cortisol responses to psychological stress: a metaanalysis. Psychoneuroendocrinology, 2005. 30(9): p. 846-56.

89. de Kloet, C.S., et al., Assessment of HPA-axis function in posttraumatic stress disorder: pharmacological and non-pharmacological challenge tests, a review. J Psychiatr Res, 2006. 40(6): p. 550-67.

90. DeRijk, R. and E.R. de Kloet, Corticosteroid receptor genetic polymorphisms and stress responsivity. Endocrine, 2005. 28(3): p. 263-70.

91. Sapolsky, R.M., L.M. Romero, and A.U. Munck, How do glucocorticoids influence stress responses? Integrating permissive, suppressive, stimulatory, and preparative actions. Endocr Rev, 2000. 21(1): p. 55-89.

92. Deroo, B.J., et al., Proteasomal inhibition enhances glucocorticoid receptor transactivation and alters its subnuclear trafficking. Mol Cell Biol, 2002. 22(12): p. 4113-23.

93. Young, E.A., S.C. Ribeiro, and W. Ye, Sex differences in ACTH pulsatility following metyrapone blockade in patients with major depression. Psychoneuroendocrinology, 2007. 32(5): p. 503-7.

94. Rohleder, N., Acute and chronic stress induced changes in sensitivity of peripheral inflammatory pathways to the signals of multiple stress systems --2011 Curt Richter Award Winner. Psychoneuroendocrinology, 2012. 37(3): p. 307-16. 
95. Roy, A. and M.K. Campbell, A unifying framework for depression: bridging the major biological and psychosocial theories through stress. Clin Invest Med, 2013. 36(4): p. E170-90. 96. Bowman, R.E., K.D. Beck, and V.N. Luine, Chronic stress effects on memory: sex differences in performance and monoaminergic activity. Horm Behav, 2003. 43(1): p. 48-59. 97. Francis, G.S., Interaction of the sympathetic nervous system and electrolytes in congestive heart failure. Am J Cardiol, 1990. 65(10): p. 24E-27E; discussion 52E.

98. Francis, G.S., Neurohormonal control of heart failure. Cleve Clin J Med, 2011. 78 Suppl 1: p. S75-9.

99. Francis, G.S., Neuroendocrine activity in congestive heart failure. Am J Cardiol, 1990. 66(11): p. 33D-38D; discussion 38D-39D.

100. Francis, G.S. and J.N. Cohn, The autonomic nervous system in congestive heart failure. Annu Rev Med, 1986. 37: p. 235-47.

101. Francis, G.S., et al., The neurohumoral axis in congestive heart failure. Ann Intern Med, 1984. 101(3): p. 370-7.

102. Francis, G.S. and W.H. Tang, Pathophysiology of congestive heart failure. Rev Cardiovasc Med, 2003. 4 Suppl 2: p. S14-20.

103. Ganguly, P.K., Role of atrial natriuretic peptide in congestive heart failure due to chronic diabetes. Can J Cardiol, 1991. 7(6): p. 275-80.

104. Frisbee, J.C., Vascular adrenergic tone and structural narrowing constrain reactive hyperemia in skeletal muscle of obese Zucker rats. Am J Physiol Heart Circ Physiol, 2006. 290(5): p. H2066-74.

105. Schreihofer, A.M., C.D. Hair, and D.W. Stepp, Reduced plasma volume and mesenteric vascular reactivity in obese Zucker rats. Am J Physiol Regul Integr Comp Physiol, 2005. 288(1): p. R253-61.

106. Esler, M., et al., Sympathetic nervous system and insulin resistance: from obesity to diabetes. Am J Hypertens, 2001. 14(11 Pt 2): p. 304S-309S.

107. Miller, A.A., K. Budzyn, and C.G. Sobey, Vascular dysfunction in cerebrovascular disease: mechanisms and therapeutic intervention. Clin Sci (Lond), 2010. 119(1): p. 1-17.

108. Peterson, E.C., Z. Wang, and G. Britz, Regulation of cerebral blood flow. Int J Vasc Med, 2011. 2011: p. 823525. 
109. Chan, S.L., et al., Pial Collateral Reactivity During Hypertension and Aging:

Understanding the Function of Collaterals for Stroke Therapy. Stroke, 2016. 47(6): p. 1618-25.

110. Black, S. and C. Iadecola, Vascular cognitive impairment: small vessels, big toll:

introduction. Stroke, 2009. 40(3 Suppl): p. S38-9.

111. Iadecola, C., V. Hachinski, and G.A. Rosenberg, Vascular cognitive impairment: introduction. Stroke, 2010. 41(10 Suppl): p. S127-8.

112. Kunz, A. and C. Iadecola, Cerebral vascular dysregulation in the ischemic brain. Handb Clin Neurol, 2009. 92: p. 283-305.

113. Iadecola, C., Vascular and Metabolic Factors in Alzheimer's Disease and Related Dementias: Introduction. Cell Mol Neurobiol, 2016. 36(2): p. 151-4.

114. Freeman, L.R. and J.N. Keller, Oxidative stress and cerebral endothelial cells: regulation of the blood-brain-barrier and antioxidant based interventions. Biochim Biophys Acta, 2012. 1822(5): p. 822-9.

115. Stanimirovic, D. and K. Satoh, Inflammatory mediators of cerebral endothelium: a role in ischemic brain inflammation. Brain Pathol, 2000. 10(1): p. 113-26.

116. Iadecola, C., Neurovascular regulation in the normal brain and in Alzheimer's disease. Nat Rev Neurosci, 2004. 5(5): p. 347-60.

117. Cipolla, M.J. and L.V. Bullinger, Reactivity of brain parenchymal arterioles after ischemia and reperfusion. Microcirculation, 2008. 15(6): p. 495-501.

118. Cipolla, M.J., et al., Increased pressure-induced tone in rat parenchymal arterioles vs. middle cerebral arteries: role of ion channels and calcium sensitivity. J Appl Physiol (1985), 2014. 117(1): p. 53-9.

119. Hotta, H., Neurogenic control of parenchymal arterioles in the cerebral cortex. Prog Brain Res, 2016. 225: p. 3-39.

120. Li, W., et al., Adaptive cerebral neovascularization in a model of type 2 diabetes: relevance to focal cerebral ischemia. Diabetes, 2010. 59(1): p. 228-35.

121. Prakash, R., et al., Cerebral neovascularization and remodeling patterns in two different models of type 2 diabetes. PLoS One, 2013. 8(2): p. e56264.

122. Prakash, R., et al., Enhanced cerebral but not peripheral angiogenesis in the Goto-Kakizaki model of type 2 diabetes involves VEGF and peroxynitrite signaling. Diabetes, 2012. 61(6): p. $1533-42$. 
123. Ainslie, P.N., R.L. Hoiland, and D.M. Bailey, Lessons from the laboratory; integrated regulation of cerebral blood flow during hypoxia. Exp Physiol, 2016.

124. Munzenmaier, D.H. and A.S. Greene, Chronic angiotensin II ATI receptor blockade increases cerebral cortical microvessel density. Am J Physiol Heart Circ Physiol, 2006. 290(2): p. H512-6.

125. Pires, P.W., et al., The effects of hypertension on the cerebral circulation. Am J Physiol Heart Circ Physiol, 2013. 304(12): p. H1598-614.

126. Ergul, A., et al., Hyperglycemia, diabetes and stroke: focus on the cerebrovasculature. Vascul Pharmacol, 2009. 51(1): p. 44-9.

127. Maneen, M.J. and M.J. Cipolla, Peroxynitrite diminishes myogenic tone in cerebral arteries: role of nitrotyrosine and F-actin. Am J Physiol Heart Circ Physiol, 2007. 292(2): p. H1042-50.

128. Zimmermann, P.A., et al., Increased myogenic tone and diminished responsiveness to ATPsensitive K+ channel openers in cerebral arteries from diabetic rats. Circ Res, 1997. 81(6): p. 996-1004.

129. Brayden, J.E. and M.T. Nelson, Regulation of arterial tone by activation of calciumdependent potassium channels. Science, 1992. 256(5056): p. 532-5.

130. Butcher, J.T., et al., Differential impact of dilator stimuli on increased myogenic activation of cerebral and skeletal muscle resistance arterioles in obese zucker rats. Microcirculation, 2013. 20(7): p. 579-89.

131. Cipolla, M.J., et al., Reperfusion decreases myogenic reactivity and alters middle cerebral artery function after focal cerebral ischemia in rats. Stroke, 1997. 28(1): p. 176-80.

132. Coulson, R.J., et al., Effects of ischemia and myogenic activity on active and passive mechanical properties of rat cerebral arteries. Am J Physiol Heart Circ Physiol, 2002. 283(6): p. $\mathrm{H} 2268-75$.

133. Jarajapu, Y.P., et al., Myogenic tone and reactivity of cerebral arteries in type II diabetic BBZDR/Wor rat. Eur J Pharmacol, 2008. 579(1-3): p. 298-307.

134. Filosa, J.A., et al., Local potassium signaling couples neuronal activity to vasodilation in the brain. Nat Neurosci, 2006. 9(11): p. 1397-1403. 
135. Hannah, R.M., et al., Endothelial $S K(\mathrm{Ca})$ and $I K(\mathrm{Ca})$ channels regulate brain parenchymal arteriolar diameter and cortical cerebral blood flow. J Cereb Blood Flow Metab, 2011. 31(5): p. 1175-86.

136. Faraci, F.M., C. Lynch, and K.G. Lamping, Responses of cerebral arterioles to ADP: eNOS-dependent and eNOS-independent mechanisms. Am J Physiol Heart Circ Physiol, 2004. 287(6): p. H2871-6.

137. Straub, S.V., et al., Regulation of intracerebral arteriolar tone by K(v) channels: effects of glucose and PKC. Am J Physiol Cell Physiol, 2009. 297(3): p. C788-96.

138. Butcher, J.T., A.G. Goodwill, and J.C. Frisbee, The ex vivo isolated skeletal microvessel preparation for investigation of vascular reactivity. J Vis Exp, 2012(62).

139. Pechanova, O. and F. Simko, The role of nitric oxide in the maintenance of vasoactive balance. Physiol Res, 2007. 56 Suppl 2: p. S7-S16.

140. Fouyas, I.P., et al., Cerebrovascular responsiveness to NG-nitro-L-arginine methyl ester in spontaneously diabetic rats. Br J Pharmacol, 1996. 118(2): p. 243-8.

141. Bohm, F. and J. Pernow, The importance of endothelin-1 for vascular dysfunction in cardiovascular disease. Cardiovasc Res, 2007. 76(1): p. 8-18.

142. Aleixandre de Artinano, A. and M. Miguel Castro, Experimental rat models to study the metabolic syndrome. Br J Nutr, 2009. 102(9): p. 1246-53.

143. Tofovic, S.P. and E.K. Jackson, Rat models of the metabolic syndrome. Methods Mol Med, 2003. 86: p. 29-46.

144. Portha, B., et al., The GK rat: a prototype for the study of non-overweight type 2 diabetes. Methods Mol Biol, 2012.933: p. 125-59.

145. Abdelsaid, M., et al., Metformin treatment in the period after stroke prevents nitrative stress and restores angiogenic signaling in the brain in diabetes. Diabetes, 2015. 64(5): p. 1804-17. 146. Schwaninger, R.M., H. Sun, and W.G. Mayhan, Impaired nitric oxide synthase-dependent dilatation of cerebral arterioles in type II diabetic rats. Life Sci, 2003. 73(26): p. 3415-25. 147. Mayhan, W.G., Impairment of endothelium-dependent dilatation of cerebral arterioles during diabetes mellitus. Am J Physiol, 1989. 256(3 Pt 2): p. H621-5.

148. Erdos, B., et al., Cerebrovascular dysfunction in Zucker obese rats is mediated by oxidative stress and protein kinase C. Diabetes, 2004. 53(5): p. 1352-9. 
149. Phillips, S.A., F.A. Sylvester, and J.C. Frisbee, Oxidant stress and constrictor reactivity impair cerebral artery dilation in obese Zucker rats. Am J Physiol Regul Integr Comp Physiol, 2005. 288(2): p. R522-30.

150. Erdos, B., et al., Rosuvastatin improves cerebrovascular function in Zucker obese rats by inhibiting NAD $(P) H$ oxidase-dependent superoxide production. Am J Physiol Heart Circ Physiol, 2006. 290(3): p. H1264-70.

151. Mayhan, W.G., et al., Exercise training normalizes impaired NOS-dependent responses of cerebral arterioles in type 1 diabetic rats. Am J Physiol Heart Circ Physiol, 2011. 300(3): p. H1013-20.

152. Miller, A.A., et al., NADPH oxidase activity and function are profoundly greater in cerebral versus systemic arteries. Circ Res, 2005. 97(10): p. 1055-62.

153. Mayhan, W.G., et al., Age-related alterations in reactivity of cerebral arterioles: role of oxidative stress. Microcirculation, 2008. 15(3): p. 225-36.

154. Modrick, M.L., et al., Role of oxidative stress and AT1 receptors in cerebral vascular dysfunction with aging. Am J Physiol Heart Circ Physiol, 2009. 296(6): p. H1914-9.

155. Doughan, A.K., D.G. Harrison, and S.I. Dikalov, Molecular mechanisms of angiotensin IImediated mitochondrial dysfunction: linking mitochondrial oxidative damage and vascular endothelial dysfunction. Circ Res, 2008. 102(4): p. 488-96.

156. Lund, D.D., et al., Role of angiotensin II in endothelial dysfunction induced by lipopolysaccharide in mice. Am J Physiol Heart Circ Physiol, 2007. 293(6): p. H3726-31. 157. Arrick, D.M., et al., Losartan improves impaired nitric oxide synthase-dependent dilatation of cerebral arterioles in type 1 diabetic rats. Brain Res, 2008. 1209: p. 128-35.

158. Trauernicht, A.K., et al., Enalapril prevents impaired nitric oxide synthase-dependent dilatation of cerebral arterioles in diabetic rats. Stroke, 2003. 34(11): p. 2698-703.

159. Ribeiro-Oliveira, A., Jr., et al., The renin-angiotensin system and diabetes: an update. Vasc Health Risk Manag, 2008. 4(4): p. 787-803.

160. Paravicini, T.M., et al., Increased NADPH-oxidase activity and Nox4 expression during chronic hypertension is associated with enhanced cerebral vasodilatation to NADPH in vivo. Stroke, 2004. 35(2): p. 584-9.

161. Lynch, C.M., et al., Nox2-derived superoxide contributes to cerebral vascular dysfunction in diet-induced obesity. Stroke, 2013. 44(11): p. 3195-201. 
162. Freed, J.K. and D.D. Gutterman, Mitochondrial reactive oxygen species and vascular function: less is more. Arterioscler Thromb Vasc Biol, 2013. 33(4): p. 673-5.

163. Schreurs, M.P. and M.J. Cipolla, Cerebrovascular dysfunction and blood-brain barrier permeability induced by oxidized LDL are prevented by apocynin and magnesium sulfate in female rats. J Cardiovasc Pharmacol, 2014. 63(1): p. 33-9.

164. Park, L., et al., Nox2-derived reactive oxygen species mediate neurovascular dysregulation in the aging mouse brain. J Cereb Blood Flow Metab, 2007. 27(12): p. 1908-18.

165. Girouard, H., et al., Cerebrovascular nitrosative stress mediates neurovascular and endothelial dysfunction induced by angiotensin II. Arterioscler Thromb Vasc Biol, 2007. 27(2): p. 303-9.

166. Kazama, K., et al., Angiotensin II impairs neurovascular coupling in neocortex through NADPH oxidase-derived radicals. Circ Res, 2004. 95(10): p. 1019-26.

167. Kitayama, J., et al., Cerebral vascular dysfunction during hypercholesterolemia. Stroke, 2007. 38(7): p. 2136-41.

168. Miller, A.A., et al., Augmented superoxide production by Nox2-containing NADPH oxidase causes cerebral artery dysfunction during hypercholesterolemia. Stroke, 2010. 41(4): p. 784-9. 169. Guzik, T.J., et al., Mechanisms of increased vascular superoxide production in human diabetes mellitus: role of $N A D(P) H$ oxidase and endothelial nitric oxide synthase. Circulation, 2002. 105(14): p. 1656-62.

170. Crack, P.J. and J.M. Taylor, Reactive oxygen species and the modulation of stroke. Free Radic Biol Med, 2005. 38(11): p. 1433-44.

171. Kelly-Cobbs, A.I., et al., Targets of vascular protection in acute ischemic stroke differ in type 2 diabetes. Am J Physiol Heart Circ Physiol, 2013. 304(6): p. H806-15.

172. Olmez, I. and H. Ozyurt, Reactive oxygen species and ischemic cerebrovascular disease. Neurochem Int, 2012. 60(2): p. 208-12.

173. Basuroy, S., et al., Nox4 NADPH oxidase mediates oxidative stress and apoptosis caused by TNF-alpha in cerebral vascular endothelial cells. Am J Physiol Cell Physiol, 2009. 296(3): p. C422-32.

174. Bedard, K. and K.H. Krause, The NOX family of ROS-generating NADPH oxidases: physiology and pathophysiology. Physiol Rev, 2007. 87(1): p. 245-313. 
175. Kuroda, J., et al., Nox4 is a major source of superoxide production in human brain pericytes. J Vasc Res, 2014. 51(6): p. 429-38.

176. Nauseef, W.M., Assembly of the phagocyte NADPH oxidase. Histochem Cell Biol, 2004. 122(4): p. 277-91.

177. Park, J.J., et al., Prognostic value of neutrophil to lymphocyte ratio in patients presenting with ST-elevation myocardial infarction undergoing primary percutaneous coronary intervention. Am J Cardiol, 2013. 111(5): p. 636-42.

178. Csiszar, A., et al., Inflammation and endothelial dysfunction during aging: role of NFkappaB. J Appl Physiol (1985), 2008. 105(4): p. 1333-41.

179. Arenillas, J.F., M.A. Moro, and A. Davalos, The metabolic syndrome and stroke: potential treatment approaches. Stroke, 2007. 38(7): p. 2196-203.

180. Landmesser, U., et al., Oxidation of tetrahydrobiopterin leads to uncoupling of endothelial cell nitric oxide synthase in hypertension. J Clin Invest, 2003. 111(8): p. 1201-9.

181. Knight, S.F., et al., Endothelial dysfunction and the development of renal injury in spontaneously hypertensive rats fed a high-fat diet. Hypertension, 2008. 51(2): p. 352-9.

182. De Martin, R., et al., The transcription factor NF-kappa B and the regulation of vascular cell function. Arterioscler Thromb Vasc Biol, 2000. 20(11): p. E83-8.

183. Singer, G. and D.N. Granger, Inflammatory responses underlying the microvascular dysfunction associated with obesity and insulin resistance. Microcirculation, 2007. 14(4-5): p. 375-87.

184. Goodwill, A.G., et al., Impact of chronic anticholesterol therapy on development of microvascular rarefaction in the metabolic syndrome. Microcirculation, 2009. 16(8): p. 667-84. 185. Pretnar-Oblak, J., et al., Influence of atorvastatin treatment on L-arginine cerebrovascular reactivity and flow-mediated dilatation in patients with lacunar infarctions. Stroke, 2006.

37(10): p. 2540-5.

186. Park, J.S., et al., Cerebral arterial pulsatility and insulin resistance in type 2 diabetic patients. Diabetes Res Clin Pract, 2008. 79(2): p. 237-42.

187. Wong, R.H., et al., Low dose resveratrol improves cerebrovascular function in type 2 diabetes mellitus. Nutr Metab Cardiovasc Dis, 2016. 26(5): p. 393-9.

188. Wong, R.H., et al., Chronic resveratrol consumption improves brachial flow-mediated dilatation in healthy obese adults. J Hypertens, 2013. 31(9): p. 1819-27. 
189. Wong, R.H., et al., Acute resveratrol supplementation improves flow-mediated dilatation in overweight/obese individuals with mildly elevated blood pressure. Nutr Metab Cardiovasc Dis, 2011. 21(11): p. 851-6.

190. Baur, J.A. and D.A. Sinclair, Therapeutic potential of resveratrol: the in vivo evidence. Nat Rev Drug Discov, 2006. 5(6): p. 493-506.

191. Knight, R.A., et al., Acute blood-brain barrier opening in experimentally induced focal cerebral ischemia is preferentially identified by quantitative magnetization transfer imaging. Magn Reson Med, 2005. 54(4): p. 822-32.

192. Abdelsaid, M., M. Coucha, and A. Ergul, Cerebrovasculoprotective effects of azilsartan medoxomil in diabetes. Transl Res, 2014. 164(5): p. 424-32.

193. Frisbee, J.C., et al., Distinct temporal phases of microvascular rarefaction in skeletal muscle of obese Zucker rats. Am J Physiol Heart Circ Physiol, 2014. 307(12): p. H1714-28. 194. Silvestre, J.S., et al., Post-ischaemic neovascularization and inflammation. Cardiovasc Res, 2008. 78(2): p. 242-9.

195. Beauquis, J., et al., Hippocampal neurovascular and hypothalamic-pituitary-adrenal axis alterations in spontaneously type 2 diabetic GK rats. Exp Neurol, 2010. 222(1): p. 125-34. 196. Renna, N.F., N. de Las Heras, and R.M. Miatello, Pathophysiology of vascular remodeling in hypertension. Int J Hypertens, 2013. 2013: p. 808353.

197. Sonoyama, K., et al., Vascular remodeling: implications for small artery function and target organ damage. Ther Adv Cardiovasc Dis, 2007. 1(2): p. 129-37.

198. Henrich, H.A., et al., Capillary rarefaction characteristic of the skeletal muscle of hypertensive patients. Klin Wochenschr, 1988. 66(2): p. 54-60.

199. Osmond, J.M., et al., Obesity increases blood pressure, cerebral vascular remodeling, and severity of stroke in the Zucker rat. Hypertension, 2009. 53(2): p. 381-6.

200. Osmond, J.M., J.D. Mintz, and D.W. Stepp, Preventing increased blood pressure in the obese Zucker rat improves severity of stroke. Am J Physiol Heart Circ Physiol, 2010. 299(1): p. H55-61.

201. Iadecola, C. and J. Anrather, Stroke research at a crossroad: asking the brain for directions. Nat Neurosci, 2011. 14(11): p. 1363-8.

202. Iadecola, C. and J. Anrather, The immunology of stroke: from mechanisms to translation. Nat Med, 2011. 17(7): p. 796-808. 
203. Frisbee, J.C., et al., Altered mechanisms underlying hypoxic dilation of skeletal muscle resistance arteries of hypertensive versus normotensive Dahl rats. Microcirculation, 2001. 8(2): p. 115-27.

204. Goodwill, A.G., M.E. James, and J.C. Frisbee, Increased vascular thromboxane generation impairs dilation of skeletal muscle arterioles of obese Zucker rats with reduced oxygen tension. Am J Physiol Heart Circ Physiol, 2008. 295(4): p. H1522-8.

205. Stapleton, P.A., et al., Altered mechanisms of endothelium-dependent dilation in skeletal muscle arterioles with genetic hypercholesterolemia. Am J Physiol Regul Integr Comp Physiol, 2007. 293(3): p. R1110-9.

206. Winocur, G., et al., Memory impairment in obese Zucker rats: an investigation of cognitive function in an animal model of insulin resistance and obesity. Behav Neurosci, 2005. 119(5): p. 1389-95.

207. Greenwood, C.E. and G. Winocur, Cognitive impairment in rats fed high-fat diets: a specific effect of saturated fatty-acid intake. Behav Neurosci, 1996. 110(3): p. 451-9.

208. Kanoski, S.E., et al., The effects of energy-rich diets on discrimination reversal learning and on BDNF in the hippocampus and prefrontal cortex of the rat. Behav Brain Res, 2007. 182(1): p. 57-66.

209. Jurdak, N., A.H. Lichtenstein, and R.B. Kanarek, Diet-induced obesity and spatial cognition in young male rats. Nutr Neurosci, 2008. 11(2): p. 48-54.

210. Zhang, L., et al., A rat model of mild cognitive impairment associated with vascular factor. Neuropathology, 2011.31(2): p. 112-21.

211. Fukui, K., et al., Cognitive impairment of rats caused by oxidative stress and aging, and its prevention by vitamin E. Ann N Y Acad Sci, 2002. 959: p. 275-84.

212. Gage, F.H., P.A. Kelly, and A. Bjorklund, Regional changes in brain glucose metabolism reflect cognitive impairments in aged rats. J Neurosci, 1984. 4(11): p. 2856-65.

213. Eskelinen, M.H., et al., Fat intake at midlife and cognitive impairment later in life: a population-based CAIDE study. Int J Geriatr Psychiatry, 2008. 23(7): p. 741-7.

214. Morris, M.C., et al., Dietary fat intake and 6-year cognitive change in an older biracial community population. Neurology, 2004. 62(9): p. 1573-9.

215. American Stroke Association. What You Should Know About Cerebral Aneurysms. 2012 [cited 2016; Available from: 
ttp://www.strokeassociation.org/STROKEORG/AboutStroke/TypesofStroke/HemorrhagicBleeds /What-You-Should-Know-About-CerebralAneurysms_UCM_310103_Article.jsp\#.V5uuyHpvkwe.

216. Diaz, O. and L. Rangel-Castilla, Endovascular treatment of intracranial aneurysms. Handb Clin Neurol, 2016. 136: p. 1303-9.

217. Nasr, D.M. and R.D. Brown, Jr., Management of Unruptured Intracranial Aneurysms. Curr Cardiol Rep, 2016. 18(9): p. 86.

218. Kim, T., et al., Incidence and risk factors of intracranial aneurysm: A national cohort study in Korea. Int J Stroke, 2016.

219. Kilic, T. and A. Akakin, Anatomy of cerebral veins and sinuses. Front Neurol Neurosci, 2008. 23: p. 4-15.

220. Uddin, M.A., T.U. Haq, and M.Z. Rafique, Cerebral venous system anatomy. J Pak Med Assoc, 2006. 56(11): p. 516-9.

221. Johansson, B.B., The Venous Blood-Brain Barrier, in The Cerebral Veins: An Experimental and Clinical Update, L.M. Auer and F. Loew, Editors. 1983, Springer Vienna: Vienna. p. 101104.

222. dela Paz, N.G. and P.A. D'Amore, Arterial versus venous endothelial cells. Cell Tissue Res, 2009. 335(1): p. 5-16.

223. Ransohoff, R.M., P. Kivisakk, and G. Kidd, Three or more routes for leukocyte migration into the central nervous system. Nat Rev Immunol, 2003. 3(7): p. 569-81.

224. Stam, J., Thrombosis of the Cerebral Veins and Sinuses. New England Journal of Medicine, 2005. 352(17): p. 1791-1798.

225. Louveau, A., et al., Structural and functional features of central nervous system lymphatic vessels. Nature, 2015. 523(7560): p. 337-41. 


\section{CHAPTER TWO:}

\section{CEREBRAL CORTICAL MICROVASCULAR RAREFACTION IN METABOLIC SYNDROME IS DEPENDENT ON INSULIN RESISTANCE AND LOSS OF NITRIC OXIDE BIOAVAILABILITY}

Paul D. Chantler ${ }^{1,6,7}$, Carl D. Shrader ${ }^{2,6,7}$, Lawrence E. Tabone ${ }^{3,6,7}$, Alexandre C. d'Audiffret ${ }^{4,6,7}$, Steven D. Brooks ${ }^{5,7}$, Kayla W. Branyan ${ }^{1,7}$, Kristen A. Grogg ${ }^{7}$, and Jefferson C. Frisbee ${ }^{5,6,7}$

Division of Exercise Physiology ${ }^{1}$, Department of Family Medicine ${ }^{2}$, Division of Bariatric Surgery $^{3}$, Division of Vascular Surgery ${ }^{4}$, Department of Physiology and Pharmacology ${ }^{5}$, Clinical and Translational Sciences Institute ${ }^{6}$, and Center for Cardiovascular and Respiratory Sciences ${ }^{7}$, West Virginia University Health Sciences Center, Morgantown, WV

Running Head: Metabolic syndrome and cerebrovascular rarefaction

Send Correspondence to:

Jefferson C. Frisbee, Ph.D.

Center for Cardiovascular and Respiratory Sciences

Department of Physiology and Pharmacology

Robert C. Byrd Health Sciences Center

PO Box 9105

West Virginia University School of Medicine

Morgantown, WV 26505

Phone: (304) 293-6527

Fax: (304) 293-5513

Email: jefrisbee@ hsc.wvu.edu

Published originally in Microcirculation, reprinted here with permission of publisher

(C) 2015 John Wiley \& Sons Ltd

Authorship Statement: SD Brooks contributed to this manuscript in terms of idea synthesis, data collection and data analysis, manuscript writing (results), and manuscript review. 


\section{ABSTRACT}

Objective: Chronic presentation of the MS is associated with an increased likelihood for stroke and poor stroke outcomes following occlusive cerebrovascular events. However, the physiological mechanisms contributing to compromised outcomes remain unclear, and the degree of cerebral cortical MVD may represent a central determinant of stroke outcomes.

Methods: This study used the OZR model of MS and clinically relevant, chronic interventions to determine the impact on cerebral cortical microvascular rarefaction via immunohistochemistry with a parallel determination of cerebrovascular function to identify putative mechanistic contributors.

Results: OZR exhibited a progressive rarefaction (to $~ 80 \%$ control MVD) of the cortical microvascular networks vs. lean Zucker rats. Chronic treatment with antihypertensive agents (captopril/hydralazine) had limited effectiveness in blunting rarefaction, although treatments improving glycemic control (metformin/rosiglitazone) were superior, maintaining $94 \%$ control MVD. Chronic treatment with the antioxidant TEMPOL severely blunted rarefaction in OZR, although this ameliorative effect was prevented by concurrent NOS inhibition.

Conclusions: Further analyses revealed that the maintenance of glycemic control and vascular NO bioavailability were stronger predictors of cerebral cortical MVD in OZR than was prevention of hypertension, and this may have implications for chronic treatment of CVD risk under stroke-prone conditions.

Key Words: obesity, capillary density, rodent models of cardiovascular disease risk, perfusion Abbreviations used: CVD, cardiovascular disease; eNOS, endothelial nitric oxide synthase; LNAME, L-NG-nitroarginine methyl ester; MCA, middle cerebral artery; MS, metabolic syndrome; MVD, microvascular density; NO, nitric oxide; OZR, obese Zucker rat; PSS, physiological salt solution. 


\section{INTRODUCTION}

With the increasing prevalence of overweight and obesity in developed economies, and the ensuing elevations to the risk for development of other associated systemic pathologies (e.g., impaired glycemic control, hypertension, atherogenic dyslipidemia), the aggregate risk for developing peripheral vascular disease is continuing at alarmingly high levels (3, 10, 29). Even more concerning is that the incidence and prevalence of these conditions is continuing to rise in our pediatric populations (34), thus resulting in the earlier development of increased risk and the elevated likelihood of poor cardiovascular outcomes at younger ages. These negative outcomes can include episodes of intermittent or chronic ischemia, poor perfusion distribution within afflicted organs or tissues (13), and acute events including myriad thromboembolytic events (e.g., stroke; 7, 25). As such, the effective interrogation and use of appropriate animal models to fully understand the organ-specific vasculopathies that are associated with the development of the metabolic syndrome is critical.

The obese Zucker rat (OZR; fa/fa) represents an animal model for the study of the cardiovascular outcomes of the metabolic syndrome with excellent utility. Similar to that in many afflicted humans, OZR develop metabolic syndrome as a result of a chronic hyperphagia and excess caloric intake based in leptin resistance and impaired satiety (1), and sequentially develop the systemic phenotype listed above to appropriate levels of severity (43).

Given the devastating potential for poor stroke outcomes on both patient quality of life and economic costs that must be borne by society, an accurate understanding of the alterations to the cerebral circulation and perfusion within the environment of the metabolic syndrome is critical. Two recent studies by Stepp and colleagues have provided initial evidence that the severity of poor stroke outcomes in the OZR was elevated as a result of the presence of the 
metabolic syndrome (32) and that this could be blunted somewhat through effective management of hypertension (31). However, these were initial studies and did not provide significant insight into the contributing elements to the poor stroke outcomes.

A critical element underlying tissue/organ viability is microvessel density (MVD), owing to its central importance in terms of maintaining an optimal environment for effective mass transport and exchange. However, recent evidence provided by our laboratory studying skeletal muscle $(12,14,15)$ and by others studying myocardium (42) and the kidney (18) provide compelling evidence that microvessel density is significantly reduced in the OZR manifesting the metabolic syndrome and that this can represent a key contributor to poor tissue/organ function and outcomes. The general purpose of this study is to not only determine the extent to which this rarefaction of the microvascular networks in the cerebral cortex is present in the OZR manifesting the full metabolic syndrome, but also to gain insight into its temporal development and the key contributing mechanisms that warrant further interrogation. Given previous results cited above, the hypothesis tested in this study was that the cerebral microcirculation of OZR undergoes a progressive reduction to microvessel density that tracks the severity of the metabolic syndrome, and that controlling hypertension in the OZR would at least partially alleviate the severity of this rarefaction.

\section{MATERIALS AND METHODS}

Animals: 6 week old male lean Zucker rats and OZR, purchased from Harlan (Indianapolis, IN), were fed standard chow and tap water ad libitum were used for all experiments unless otherwise stated (see below). Rats were housed in animal care facility at the West Virginia University Health Sciences Center to the appropriate age range, and all protocols received prior IACUC approval. At the time of final usage, rats were anesthetized with injections of sodium pentobarbital $(50 \mathrm{mg} / \mathrm{kg}$ 
i.p.), and received tracheal intubation to facilitate maintenance of a patent airway. In all rats a carotid artery and an external jugular vein were cannulated for determination of arterial pressure and for intravenous infusion of additional substances as necessary (e.g., anesthetic, heparin, etc.).

LZR and OZR were used at three distinct age groups, 7-8 weeks, 12-13 weeks and 16-17 weeks of age. These age ranges were chosen to encompass the significant phases of development of the metabolic syndrome in OZR (4). At 7-8 weeks, OZR are obese, and exhibit a moderate degree of insulin resistance

with minimal fasting hyperglycemia or elevated mean arterial pressure. At 12-13 weeks, OZR experience a pronounced insulin resistance and manifest a mild elevation in mean arterial pressure. By 16-17 weeks of age, OZR are severely insulin-resistant, begin to demonstrate moderate fasting hyperglycemia and exhibit a moderate hypertension.

Starting at 7 weeks of age, rats were placed in one of several groups to the time of final usage:

1. Time control (normal food and water ad libitum)

2. Anti-hypertensive treatment with captopril (angiotensin converting enzyme inhibitor; 60 $\mathrm{mg} / \mathrm{kg} /$ day; drinking water; $[14,20])$

3. Anti-hypertensive treatment with hydralazine (systemic vasodilator; $50 \mathrm{mg} / \mathrm{kg} / \mathrm{day}$; drinking water; $[14,20])$

4. Anti-diabetic treatment with metformin (hepatic gluconeogenesis inhibitor; $300 \mathrm{mg} / \mathrm{kg} / \mathrm{day}$; drinking water; $[20,45])$

5. Anti-diabetic treatment with rosiglitazone (insulin sensitizing agent; $10 \mathrm{mg} / \mathrm{kg} / \mathrm{day}$; mixed with food; $[20,37])$ 
6. Inhibition of endothelial nitric oxide synthase (eNOS) with L-NG-Nitroarginine Methyl Ester (LNAME; 100 mg/kg/day; drinking water; [15])

7. Anti-oxidant treatment with TEMPOL $\left(10^{-3} \mathrm{M}\right.$; drinking water [15])

8. Combined inhibition of eNOS (L-NAME) and anti-oxidant (TEMPOL) treatment

Investigation of Isolated Vessels: Following the initial surgery, each rat was decapitated and the brain removed from the skull case and placed in cold physiological salt solution (PSS; $4^{\circ} \mathrm{C}$ ). Subsequently, a middle cerebral artery (MCA) was dissected from its origin at the Circle of Willis, as described previously $(5,8)$. Arteries were placed in a heated chamber $\left(37^{\circ} \mathrm{C}\right)$ that allowed the lumen and exterior of the vessel to be perfused and superfused, respectively, with PSS equilibrated with $21 \% \mathrm{O}_{2}, 5 \% \mathrm{CO}_{2} ; 74 \% \mathrm{~N}_{2}$ from separate reservoirs. Vessels were cannulated at both ends with glass micropipettes and were tied (10-0 nylon suture) to the inflow and outflow pipettes which were connected to a reservoir perfusion system that allowed intralumenal pressure and gas concentrations to be controlled. Any side branches were ligated using a single strand teased from 6-0 suture. Vessel diameter was measured using television microscopy and an on-screen video micrometer. Arteries were extended to their in situ length and were equilibrated at $80 \%$ of the animal's mean arterial pressure ( $\sim 82 \mathrm{mmHg}$ for LZR; 100 $\mathrm{mmHg}$ for OZR). Active tone for pressurized MCA in the present study, calculated as $\left(\Delta D / D_{\max }\right) \cdot 100$, where $\Delta \mathrm{D}$ is the diameter increase from rest in response to $\mathrm{Ca}^{2+}$-free PSS, and $\mathrm{D}_{\max }$ is the maximum diameter measured at the equilibration pressure in $\mathrm{Ca}^{2+}$-free PSS, averaged $31 \pm 3 \%$ in LZR and $32 \pm 4 \%$ in OZR. The reactivity of isolated arteries was assessed in response to increasing concentrations of acetylcholine $\left(10^{-10} \mathrm{M}-10^{-6} \mathrm{M}\right)$. To determine the extent to which vascular nitric oxide bioavailability contributes to responses to acetylcholine in the MCA at the different ages and under the differed conditions of the present study, all vessels were 
treated acutely with L-NAME $\left(10^{-4} \mathrm{M}\right.$; Sigma) following their responses under control conditions to abolish the contribution of nitric oxide synthase.

Measurement of Vascular NO Bioavailability: From each rat, the abdominal aorta was removed and vascular nitric oxide (NO) production was assessed using amperometric sensors (World Precision Instruments, Sarasota, FL). Briefly, aortae were isolated, sectioned longitudinally, pinned in a silastic coated dish and superfused with warmed $\left(37^{\circ} \mathrm{C}\right)$ PSS equilibrated with $95 \%$ $\mathrm{O}_{2}$ and $5 \% \mathrm{CO}_{2}$. An NO sensor (ISO-NOPF 100) was placed in close apposition to the endothelial surface and a baseline level of current was obtained. Subsequently, increasing concentrations of methacholine $\left(10^{-10}-10^{-6} \mathrm{M}\right)$ were added to the bath and the changes in current were determined. To verify that responses represented NO release, these procedures were repeated following pre-treatment of aortic strip with L-NAME $\left(10^{-4} \mathrm{M}\right)$.

Determination of Microvessel Density: Following removal of the MCAs from the Circle of Willis on the base of the brain, the brain was placed within Tissue-Tek OCT compound and frozen. Brains were then sliced into $5 \mu \mathrm{m}$ cross sections and where then stained using the established approach developed by Munzenmaier and Greene (30) using primary anti-CD-31 antibody. Under microscopy, localization of labeled microvessels was performed with a Nikon E600 upright microscope with a 20x objective lens. The microscope was coupled to cooled CCD camera (Micromax; Princeton Instruments Inc, Trenton, NJ). Five nearby $1 \mathrm{~mm}^{2}$ images were taken from each of three sections in the frontal cortex of each brain, and the mean microvessel density within these 15 images was taken to represent cortical MVD in that animal. All acquired images from individual sections were analyzed for number of microvessels using MetaMorph Imaging software (Universal Imaging Co., Downingtown, PA). 
Data and Statistical Analyses: All data are presented as mean \pm SEM. Statistically significant differences in microvessel density measurements, slope coefficients, and measurements of plasma biomarkers were determined using analysis of variance (ANOVA). In all cases, Student-NewmanKeuls post hoc test was used when appropriate and $\mathrm{p}<0.05$ was taken to reflect statistical significance. The mechanical responses of isolated MCA following acetylcholine challenge were fit with the three-parameter logistic equation:

$$
y=\min +\left[\frac{\max -\min }{1+10^{\log E D_{50}-x}}\right]
$$

where $y$ represents the change in arteriolar diameter, "min" and "max" represent the lower and upper bounds, respectively, of the change in arteriolar diameter with increasing acetylcholine concentration, $x$ is the logarithm of the acetylcholine concentration and $\log E D_{50}$ represents the logarithm of the acetylcholine concentration ( $x$ ) at which the response $(y)$ is halfway between the lower and upper bounds.

Vascular NO bioavailability measurements were fit with a linear regression equation $\left(y=\alpha_{0}+\beta_{1} x\right)$; where $y$ represents the NO concentration, $\alpha_{0}$ represents an intercept term, $\beta_{1}$ represents the slope of the relationship, and $x$ represents the log molar concentration of methacholine.

\section{RESULTS}

Data describing the baseline characteristics of LZR and OZR under the conditions of the present study are presented in Table 1 . Throughout the age ranges used in the present study, OZR were heavier than LZR and also demonstrated a progressive worsening of glycemic control and an elevation in mean arterial pressure. Chronic treatment with captopril and hydralazine was effective 
at preventing the development of hypertension and captopril was also marginally effective at improving insulin resistance. Treatment with either metformin or rosiglitazone significantly improved glycemic control, and had modest effects on blunting the development of elevated blood pressure. Chronic antioxidant therapy also had modest effects on reducing hypertension and insulin resistance.

Figure 1 presents the changes in cerebral cortical MVD in LZR and OZR over the age ranges of the current study. While there was no evidence for microvascular rarefaction at 7-8 weeks of age, and a modest, but statistically significant, reduction in MVD by 12-13 weeks, cortical MVD was reduced by approximately $20 \%$ in $16-17$ week old OZR as compared to levels in age-matched LZR.

The effects of chronic anti-hypertensive treatment on the changes in cortical MVD are summarized in Figure 2. While both captopril and hydralazine were comparable in terms of their efficacy in preventing the development of hypertension, only treatment with captopril resulted in a significant improvement to the levels of cerebral MVD in OZR as compared to levels in the untreated control animals.

Figure 3 presents the data describing the effects of chronic treatments to improve glycemic control on cerebral MVD in LZR and OZR. Treatment with either metformin or rosiglitazone resulted in a significant improvement to MVD in OZR as compared to responses in untreated controls. No differences between metformin and rosiglitazone treatment in terms of the impact on rarefaction were evident in OZR.

Data describing the effects of chronic eNOS blockade and/or antioxidant treatment on cerebral cortex MVD in LZR and OZR are summarized in Figure 4. Chronic treatment with LNAME appeared to accelerate the cerebrovascular rarefaction in OZR, but did not result in a 
significant change in the final magnitude of the reduced MVD. In contrast, chronic treatment with TEMPOL significantly reduced the severity of the rarefaction in OZR, such that the only significant reduction to MVD occurred at 16-17 weeks of age. Combined treatment with LNAME and TEMPOL resulted in a similar change in MVD as with L-NAME alone, abolishing the beneficial impact of the chronic TEMPOL treatment.

Figure 5 summarizes the vascular NO bioavailability, assessed using conduit arteries and the amperometric NO sensors following challenge with increasing concentrations of methacholine. At the youngest age range, vascular NO bioavailability was very comparable between LZR and OZR, and the brief imposition ( 1 week) of the pharmacological regimens, with the singular exception of L-NAME, did not have a significant impact of methacholineinduced NO bioavailability (Panel A). At 12-13 weeks of age, vascular NO bioavailability was significantly reduced in OZR as compared to LZR, although this loss was blunted by treatment with captopril, metformin, rosiglitazone or TEMPOL (Panel B). These effects were mirrored in OZR at 16-17 weeks of age, although the reduction to NO bioavailability in untreated OZR was more pronounced, such that the relative degrees of drug treatment-induced improvements was increased (Panel C).

The dilator reactivity of isolated MCA from rats in each group in response to challenge with increasing concentrations of acetylcholine are summarized in Figure 6. At the youngest age group, dilator responses of MCA to acetylcholine were not different between LZR and OZR and the impact of the chronic drug treatment groups, except for L-NAME, was negligible. Acute treatment with L-NAME severely abolished dilator responses to acetylcholine in all groups at the age range (Panel A). At the 12-13 weeks age range, MCA from OZR exhibited a significant reduction in their acetylcholine-induced dilation as compared to LZR, and impaired response 
was largely prevented by treatment with captopril, TEMPOL and the agents targeted at improving glycemic control (Panel B). In the older cohort of animals, the dilator responses of MCA to acetylcholine were severely attenuated in OZR as compared to LZR and all drug treatments, with the exception of chronic L-NAME, resulted in a significant improvement to reactivity, with TEMPOL, metformin and rosiglitazone being most effective (Panel C). In all cases, at all ages, acute administration of L-NAME $\left(10^{-4} \mathrm{M}\right)$ to the vessel nearly abolished reactivity of MCA to acetylcholine (data not shown).

Figure 7 presents the correlation between group aggregate vascular NO bioavailability (from Figure 5) and group aggregate MVD (from Figures 1-4). These data suggest that there is a clear, positive correlation between estimated vascular NO bioavailability and cerebral cortex MVD across the age ranges and conditions of the present study. In LZR and OZR at 7-8 weeks of age (black), there was minimal disparity in either parameter and, as such, the data clump together. With increasing age, the progressive loss in NO bioavailability and MVD in OZR cause the data to distribute, although a clear correlation is still present, under both control conditions and following pharmacological intervention.

\section{DISCUSSION}

With the increasing incidence of the constituent pathologies of the metabolic syndrome, and the fact that this is occurring in younger individuals at a higher rate than ever before, understanding the cardiovascular outcomes of these conditions, their mechanistic bases and the potential for blunting their development or reversing their severity is a critical area for ongoing investigation. The purpose of the present study was to use the OZR model of the metabolic syndrome, which derives its origin in a severe systemic leptin resistance, to determine the extent 
to which cerebral cortical MVD is either maintained or degraded, what the fundamental mechanisms underlying these responses are, and the efficacy of physiologically and clinically relevant interventions might help to improve outcomes.

The primary observation of the present study was that, with the development of the metabolic syndrome, the cerebral cortical MVD of OZR steadily fell as compared to levels in the control LZR. Given the clear results presented in previous studies of the OZR myocardium (42), kidney (18) and skeletal muscle (12), the progressive rarefaction of the cerebral cortical microvascular networks clearly reflects a broad negative cardiovascular outcome that impacts multiple diverse organs and tissues within the metabolic syndrome. However, this did not appear to reflect the classic interpretation of microvascular rarefaction as the long term adaptation to elevated perfusion pressure $(23,40)$, as treatment with captopril or hydralazine, to minimize the development of elevated arterial pressure in OZR, had disparate effects on maintaining MVD despite comparable effectiveness in preventing elevated pressure. As such, while elevated mean arterial pressure within the metabolic syndrome in OZR may represent a significant contributor to altered vascular wall mechanics $(32,39)$ or patterns of vascular reactivity $(13)$, this does not appear to be a major contributor to the evolving microvascular rarefaction.

Comparable to an observation made several years ago in the skeletal muscle of OZR with regards to chronic treatments against the conditions of the metabolic syndrome (14), it was determined that chronic captopril treatment had modest effects at improving glycemic control in OZR in addition to its anti-hypertensive effects, and that this was associated with a blunted severity of cerebral cortical microvascular rarefaction. This observation suggests that controlling the severity of insulin-resistance and impaired glycemic control may be a more important therapeutic target than controlling blood pressure alone for this outcome. As a result, OZR were 
chronically treated with two mechanistically divergent drugs for improving glycemic control, metformin and rosiglitazone, to determine their effectiveness in terms of blunting the progressive loss in cerebral microvessel density. Interestingly, both metformin and rosiglitazone were comparably effective in improving glycemic control and in blunting the loss in MVD. This is an observation that is consistent with those from previous studies in both relevant animal models ( 2 , 6) and in human subjects afflicted with diabetes mellitus $(16,17)$, where superior maintenance of insulin sensitivity and glycemic control where key variables associated with improving MVD (where rarefaction had occurred) and attenuating its loss (where rarefaction was relatively mild).

Our previous investigative efforts into the physiological mechanisms contributing to microvascular rarefaction in skeletal muscle $(12,15)$ and those of others focusing on other tissues/organs $(18,42)$, has repeatedly implicated the balance between systemic vascular oxidant stress and endothelial function (e.g., NO bioavailability, altered arachidonic acid metabolism) as a key contributor to the progression and severity of microvascular rarefaction. The present study uses two distinct measurements to assess vascular NO bioavailability, amperometric sensors for methacholine-induced NO release and the mechanical responses of ex vivo cerebral microvessels in response to an overwhelmingly NO dependent stimulus (acetylcholine, [41]). As shown in figure 5 and 6, vascular NO bioavailability in young OZR (regardless of therapeutic intervention) was very comparable to that determined in LZR, and this was the condition wherein no significant reduction to cortical microvessel density was observed. As NO bioavailability began to fall with increasing severity of the metabolic syndrome, the severity of the rarefaction followed this, to the point where the lowest levels of cortical MVD were identified in the oldest cohort of OZR with no therapeutic intervention. Interventions that were associated with improvements to vascular NO bioavailability (e.g., captopril, metformin, rosiglitazone) were the 
ones most effective at blunting the severity of the cortical microvascular rarefaction in OZR. As a further support to this concept, chronic treatment of OZR with the anti-oxidant TEMPOL resulting in a superior maintenance of vascular NO bioavailability also exhibited a superior maintenance of microvessel density. However, any protective effect of TEMPOL was abolished if NO bioavailability was lost (e.g., combined treatment with L-NAME). Clearly, under the conditions of the present study, and for the cerebral cortical microvasculature, the levels of vascular NO bioavailability are a powerful positive correlate with MVD under the conditions of the metabolic syndrome.

Our recent study of the temporal characteristics of microvascular rarefaction in the skeletal muscle of OZR provided evidence of an early phase of microvessel loss that was NOindependent and appeared to reflect events associated with the inflammatory signaling cascades

on the venular endothelium that were strongly dependent on the production and actions of thromboxane $\mathrm{A}_{2}\left(\mathrm{TxA}_{2}\right)$ and post-capillary venular leukocyte adhesion/rolling events (12). While the results from the current study do not rule out this possibility as a potential contributing factor for the microvessel loss in the cerebral cortex of OZR, there was little evidence for the "early phase" of rarefaction in this tissue that was identified in peripheral skeletal muscle. However, future investigation into the specific initiating mechanisms for cerebral cortical microvessel loss within the metabolic syndrome is clearly warranted.

\section{Clinical and Translational Perspective}

Not only is metabolic syndrome associated with increased cardiovascular disease events and morality (27) but it is an established risk factor for cognitive decline, dementia, and stroke $(24,35,36,44,46)$. Although the mechanisms by which metabolic syndrome confers its risk have not been determined, it has been proposed that the ill effects of metabolic syndrome on 
cerebral function are, in part, due to an oxidative stress imbalance, neuro-inflammation, and impaired vascular reactivity $(19,28,38)$. We suggest that the damaging effects of metabolic syndrome on cerebral integrity are in part dependent on the progressive decline in MVD and vascular reactivity, thereby limiting cerebrovascular reserve capacity. Microvascular rarefaction affects spatial hemodynamics and induces a non-uniform blood flow distribution, and has been implicated in reducing the capillary transport of small solutes and in enhancing the risk of target organ damage $(22,26)$. Such pathological adaptations would damage cerebral auto-regulation and blood flow reserve, favoring the occurrence of cognitive impairment, and ischemic stroke.

Ameliorating or delaying the onset of these cerebral vascular alterations in metabolic syndrome would likely improve clinical outcomes. In OZR, the cerebrovascular changes correlated with the development of hypertension and greater cerebral injury after a stroke (31, 32), which would suggest that blood pressure has a key role in the cerebral damage. While we have shown that chronic blood pressure treatment with a systemic vasodilator (hydralazine) or an angiotensin converting enzyme inhibitor (captopril) were equally effective in preventing the development of hypertension, only captopril blunted the decline in cerebral cortical MVD and vascular reactivity. Further, chronic blood pressure treatment (hydrochlorothiazide) during the progression of metabolic syndrome improved vascular remodeling and prevented stoke injury $(31,32)$. Similarly, in a randomized, non-blinded, multi-center trial that evaluated the effects of intensive blood pressure lowering on cardiovascular risk in diabetic patients, identified that intensive blood pressure control lowered secondary endpoints such as the annual rate of total stroke and nonfatal stroke compared to standard therapy (21). Therefore it is possible that the blunted cerebrovascular impairment with metabolic syndrome with blood pressure control resulted in a more favorable clinical outcome. 
Interestingly, our study showed that glycemic control rather than blood pressure control appeared to be a major contributor to the microvascular remodeling. Whereby chronic glycemic control with metformin (or rosiglitazone) during the development of metabolic syndrome improved cerebral microvessel density and reactivity to a greater extent that blood pressure control. In humans, intensive glycemic control with metformin compared with conventional therapy (diet alone), led to a significantly greater risk reduction of death due to stroke (9). While metformin administered shortly after an ischemic stroke in diabetic rats improved cerebral vascular repair and cognitive function (33). Metformin exerts its anti-hyperglycemic actions by reducing hepatic gluconeogenesis and increasing peripheral glucose uptake, insulin sensitivity, and fatty acid oxidation, but importantly metformin also improves antioxidant capacity (11). Indeed chronic treatment with TEMPOL, an anti-oxidant, also improved cerebral angiogenesis and vascular reactivity in our metabolic syndrome model. Therefore, chronic glycemic control would seem to be an effective strategy that confers vascular protection, and perhaps should be administered in individuals presenting with early signs of metabolic syndrome, rather than given once full-blown metabolic syndrome or diabetes has developed. Future research should directly compare between control glucose vs. blood pressure vs. a combination therapy on preventing poor clinical outcome in the metabolic syndrome model.

Acknowledgements: The authors would like to thank Ms. Milinda James for her expert technical assistance. Additionally, we also acknowledge the support provided through Center for Cardiovascular and Respiratory Sciences and the Clinical and Translational Sciences Institute at the West Virginia University Health Sciences Center. This study was supported by the American Heart Association (IRG 14330015, PRE 16850005, EIA 0740129N), and the National Institutes of Health (U54GM104942; RR 2865AR; P20 RR 016477). 


\section{LITERATURE CITED}

1. Aleixandre de Artiñano A, Miguel Castro M. Experimental rat models to study the metabolic syndrome. Br J Nutr. 2009 Nov;102(9):1246-53.

2. Ashoff A, Qadri F, Eggers R, Jöhren O, Raasch W, Dendorfer A. Pioglitazone prevents capillary rarefaction in streptozotocin-diabetic rats independently of glucose control and vascular endothelial growth factor expression. J Vasc Res. 2012;49(3):260-6.

3. Bastien M, Poirier P, Lemieux I, Després JP. Overview of epidemiology and contribution of obesity to cardiovascular disease. Prog Cardiovasc Dis. 2014 Jan-Feb;56(4):369-81.

4. Bray GA. The Zucker-fatty rat: a review. Fed Proc. 1977 Feb;36(2):148-53.

5. Butcher JT, Goodwill AG, Frisbee JC. The ex vivo isolated skeletal microvessel preparation for investigation of vascular reactivity. J Vis Exp. 2012 Apr 28;(62). pii: 3674.

6. Cipolla MJ, Bishop N, Vinke RS, Godfrey JA. PPAR\{gamma\} activation prevents hypertensive remodeling of cerebral arteries and improves vascular function in female rats. Stroke. 2010 Jun;41(6):1266-70.

7. Di Minno MN, Tufano A, Ageno W, Prandoni P, Di Minno G. Identifying high-risk individuals for cardiovascular disease: similarities between venous and arterial thrombosis in perspective. A 2011 update. Intern Emerg Med. 2012 Feb;7(1):9-13.

8. Durand MJ, Moreno C, Greene AS, Lombard JH. Impaired relaxation of cerebral arteries in the absence of elevated salt intake in normotensive congenic rats carrying the Dahl salt-sensitive renin gene. Am J Physiol Heart Circ Physiol. 2010 Dec;299(6):H1865-74.

9. Effect of intensive blood-glucose control with metformin on complications in overweight patients with type 2 diabetes (UKPDS 34). UK Prospective Diabetes Study (UKPDS) Group. Lancet 352: 854-865, 1998.

10. Falkner B, Cossrow ND. Prevalence of metabolic syndrome and obesity-associated hypertension in the racial ethnic minorities of the United States. Curr Hypertens Rep. 2014 Jul;16(7):449.

11. Faure P, Wiernsperger N, Polge C, Favier A, and Halimi S. Impairment of the antioxidant properties of serum albumin in patients with diabetes: protective effects of metformin. Clin Sci (Lond) 114: 251-256, 2008.

12. Frisbee JC, Goodwill AG, Frisbee SJ, Butcher JT, Brock RW, Olfert IM, DeVallance ER, Chantler PD. Distinct temporal phases of microvascular rarefaction in skeletal muscle of obese Zucker rats. Am J Physiol Heart Circ Physiol. 2014 Dec 15;307(12):H1714-28. 
13. Frisbee JC, Delp MD. Vascular function in the metabolic syndrome and the effects on skeletal muscle perfusion: lessons from the obese Zucker rat. Essays Biochem. 2006;42:145-61.

14. Frisbee JC. Hypertension-independent microvascular rarefaction in the obese Zucker rat model of the metabolic syndrome. Microcirculation. 2005 Jul-Aug;12(5):383-92.

15. Frisbee JC. Reduced nitric oxide bioavailability contributes to skeletal muscle microvessel rarefaction in the metabolic syndrome. Am J Physiol Regul Integr Comp Physiol. 2005 Aug;289(2):R307-R316.

16. Gealekman O, Guseva N, Gurav K, Gusev A, Hartigan C, Thompson M, Malkani S, Corvera S. Effect of rosiglitazone on capillary density and angiogenesis in adipose tissue of normoglycaemic humans in a randomised controlled trial. Diabetologia. 2012 Oct;55(10):27949.

17. Gealekman O, Burkart A, Chouinard M, Nicoloro SM, Straubhaar J, Corvera S. Enhanced angiogenesis in obesity and in response to PPARgamma activators through adipocyte VEGF and ANGPTL4 production. Am J Physiol Endocrinol Metab. 2008 Nov;295(5):E1056-64.

18. Gealekman O, Brodsky SV, Zhang F, Chander PN, Friedli C, Nasjletti A, Goligorsky MS. Endothelial dysfunction as a modifier of angiogenic response in Zucker diabetic fat rat: amelioration with Ebselen. Kidney Int. 2004 Dec;66(6):2337-47.

19. Giannopoulos S, Boden-Albala B, Choi JH, Carrera E, Doyle M, Perez T, and Marshall RS. Metabolic syndrome and cerebral vasomotor reactivity. Eur J Neurol 17: 1457-1462, 2010.

20. Goodman and Gilman's The Pharmacological Basis of Therapeutics, 11th Edition. Eds: LL Brunton, JS LAzo, KL Parker. McGraw-Hill Professional. 2005

21. Group AS, Cushman WC, Evans GW, Byington RP, Goff DC, Jr., Grimm RH, Jr., Cutler JA, Simons-Morton DG, Basile JN, Corson MA, Probstfield JL, Katz L, Peterson KA, Friedewald WT, Buse JB, Bigger JT, Gerstein HC, and Ismail-Beigi F. Effects of intensive blood-pressure control in type 2 diabetes mellitus. N Engl J Med 362: 1575-1585, 2010.

22. Grundy SM, Cleeman JI, Daniels SR, Donato KA, Eckel RH, Franklin BA, Gordon DJ, Krauss RM, Savage PJ, Smith SC, Jr., Spertus JA, and Costa F. Diagnosis and management of the metabolic syndrome: an American Heart Association/National Heart, Lung, and Blood Institute Scientific Statement. Circulation 112: 2735-2752, 2005.

23. Henrich HA, Romen W, Heimgärtner W, Hartung E, Bäumer F. Capillary rarefaction characteristic of the skeletal muscle of hypertensive patients. Klin Wochenschr. 1988 Jan 15;66(2):54-60. 
24. Koren-Morag N, Goldbourt U, and Tanne D. Relation Between the Metabolic Syndrome and Ischemic Stroke or Transient Ischemic Attack: A Prospective Cohort Study in Patients With Atherosclerotic Cardiovascular Disease. Stroke 36: 1366-1371, 2005.

25. Kostapanos MS, Florentin M, Elisaf MS, Mikhailidis DP. Hemostatic factors and the metabolic syndrome. Curr Vasc Pharmacol. 2013 Nov;11(6):880-905.

26. Levy BI, Ambrosio G, Pries AR, and Struijker-Boudier HA. Microcirculation in hypertension: a new target for treatment? Circulation 104: 735-740, 2001.

27. Malik S, Wong ND, Franklin SS, Kamath TV, L'Italien GJ, Pio JR, and Williams GR. Impact of the Metabolic Syndrome on Mortality From Coronary Heart Disease, Cardiovascular Disease, and All Causes in United States Adults. Circulation 110: 1245-1250, 2004.

28. Marshall RS, Rundek T, Sproule DM, Fitzsimmons BF, Schwartz S, and Lazar RM. Monitoring of cerebral vasodilatory capacity with transcranial Doppler carbon dioxide inhalation in patients with severe carotid artery disease. Stroke 34: 945-949, 2003.

29. Milton K, Macniven R, Bauman A. Review of the epidemiological evidence for physical activity and health from low- and middle-income countries. Glob Public Health. 2014;9(4):36981.

30. Munzenmaier DH, Greene AS. Chronic angiotensin II AT1 receptor blockade increases cerebral cortical microvessel density. Am J Physiol Heart Circ Physiol. 2006 Feb;290(2):H5126.

31. Osmond JM, Mintz JD, Stepp DW. Preventing increased blood pressure in the obese Zucker rat improves severity of stroke. Am J Physiol Heart Circ Physiol. 2010 Jul;299(1):H55-61.

32. Osmond JM, Mintz JD, Dalton B, Stepp DW. Obesity increases blood pressure, cerebral vascular remodeling, and severity of stroke in the Zucker rat. Hypertension. 2009 Feb;53(2):3816.

33. Prakash R, Li W, Qu Z, Johnson MA, Fagan SC, and Ergul A. Vascularization Pattern After Ischemic Stroke is Different in Control Versus Diabetic Rats: Relevance to Stroke Recovery. Stroke 44: 2875-2882, 2013.

34. Prendergast C, Gidding SS. Cardiovascular risk in children and adolescents with type 2 diabetes mellitus. Curr Diab Rep. 2014 Feb;14(2):454.

35. Raffaitin C, Gin H, Empana JP, Helmer C, Berr C, Tzourio C, Portet F, Dartigues JF, Alperovitch A, and Barberger-Gateau P. Metabolic syndrome and risk for incident Alzheimer's disease or vascular dementia: the Three-City Study. Diabetes Care 32: 169-174, 2009. 
36. Razay G, Vreugdenhil A, and Wilcock G. The metabolic syndrome and Alzheimer disease. Arch Neurol 64: 93-96, 2007.

37. Sardone LD, Renlund R, Willett TL, Fantus IG, Grynpas MD. Effect of rosiglitazone on bone quality in a rat model of insulin resistance and osteoporosis. Diabetes. 2011 Dec;60(12):3271-8.

38. Silvestrini M, Vernieri F, Pasqualetti P, Matteis M, Passarelli F, Troisi E, and Caltagirone C. Impaired cerebral vasoreactivity and risk of stroke in patients with asymptomatic carotid artery stenosis. JAMA 283: 2122-2127, 2000.

39. Stepp DW, Pollock DM, Frisbee JC. Low-flow vascular remodeling in the metabolic syndrome X. Am J Physiol Heart Circ Physiol. 2004 Mar;286(3):H964-70.

40. Struyker-Boudier HA, le Noble JL, le Noble FA, Messing MW, van Essen H. Hypertension, the microcirculation and serotonin. Clin Physiol Biochem. 1990;8 Suppl 3:28-39.

41. Sylvester FA, Stepp DW, Frisbee JC, Lombard JH. High-salt diet depresses acetylcholine reactivity proximal to NOS activation in cerebral arteries. Am J Physiol Heart Circ Physiol. 2002 Jul;283(1):H353-63.

42. Toblli JE, Cao G, DeRosa G, Di Gennaro F, Forcada P. Angiotensin-converting enzyme inhibition and angiogenesis in myocardium of obese Zucker rats. Am J Hypertens. 2004 Feb;17(2):172-80.

43. Tofovic SP, Jackson EK. Rat models of the metabolic syndrome. Methods Mol Med. 2003;86:29-46.

44. Vanhanen M, Koivisto K, Moilanen L, Helkala EL, Hanninen T, Soininen H, Kervinen K, Kesaniemi YA, Laakso M, and Kuusisto J. Association of metabolic syndrome with Alzheimer disease: a population-based study. Neurology 67: 843-847, 2006.

45. Wessels B, Ciapaite J, van den Broek NM, Nicolay K, Prompers JJ. Metformin impairs mitochondrial function in skeletal muscle of both lean and diabetic rats in a dose-dependent manner. PLoS One. 2014 Jun 20;9(6):e100525.

46. Wild SH, Byrne CD, Tzoulaki I, Lee AJ, Rumley A, Lowe GD, and Fowkes FG. Metabolic syndrome, haemostatic and inflammatory markers, cerebrovascular and peripheral arterial disease: The Edinburgh Artery Study. Atherosclerosis 203: 604-609, 2009. 


\section{FIGURE LEGENDS}

Figure 1. The change in skeletal muscle microvessel density in LZR and OZR between 7-8 and 1617 weeks of age. Data are presented as mean \pm SE, $n=6$ animals in each age group for LZR; $n=7$ animals in each age group for OZR. *; $\mathrm{p}<0.05$ vs. LZR at that age. Please see text for details.

Figure 2. The change in skeletal muscle microvessel density in LZR and OZR between 7-8 and 1617 weeks of age. Data (mean \pm SE) are presented under control conditions (grey) and in response to chronic treatment with either captopril or hydralazine as an anti-hypertensive therapy. $n=6$ animals in each age group for LZR; n=5-6 animals in each age group for OZR. *; $\mathrm{p}<0.05$ vs. LZR at that age. $\uparrow ; p<0.05$ vs. OZR at that age. Please see text for details.

Figure 3. The change in skeletal muscle microvessel density in LZR and OZR between 7-8 and 1617 weeks of age. Data (mean \pm SE) are presented under control conditions (grey) and in response to chronic treatment with either metformin or rosiglitazone as therapy for improving glycemic control. $\mathrm{n}=6$ animals in each age group for LZR; $\mathrm{n}=5-6$ animals in each age group for OZR.*; $\mathrm{p}<0.05$ vs. LZR at that age. $\uparrow ; p<0.05$ vs. OZR at that age. Please see text for details.

Figure 4. The change in skeletal muscle microvessel density in LZR and OZR between 7-8 and 1617 weeks of age. Data (mean \pm SE) are presented under control conditions (grey) and in response to chronic treatment with TEMPOL, L-NAME or both agents together as means for separating oxidant stress from nitric oxide bioavailability. $n=6$ animals in each age group for LZR; $n=5-6$ animals in each age group for OZR. *; $\mathrm{p}<0.05$ vs. LZR at that age. $\uparrow ; \mathrm{p}<0.05$ vs. OZR at that age. Please see text for details. 
Figure 5. Data describing the bioavailability of vascular-produced nitric oxide from ex vivo segments of the abdominal aorta from LZR and OZR at 7-8 (Panel A), 12-13 (Panel B) and 16-17 (Panel C) weeks of age. Data are presented as the slope of the NO level with increasing concentrations of methacholine pooled arteries under control conditions and following the employed chronic interventions to the animal prior to use. Abbreviations: CAP (captopril), HDZ (hydralazine), MET (metformin), RGZ (rosiglitazone), TEM (TEMPOL), LNM (L-NAME), TLN (TEMPOL+L-NAME). Data are presented as mean \pm SE, $n=6$ animals in each age group for LZR; $\mathrm{n}=5-6$ animals in each age group for OZR. *; $\mathrm{p}<0.05$ versus responses in LZR under control conditions. $\uparrow ; \mathrm{p}<0.05$ versus responses in OZR under control conditions. Please see text for details.

Figure 6. Data describing the dilator reactivity of ex vivo middle cerebral arteries from LZR and OZR at 7-8 weeks (Panel A), 12-13 weeks (Panel B) and 16-17 weeks of age (Panel C). Data, presented for the dilator responses of MCA in response to increasing concentrations of acetylcholine, are shown as mean $\pm \mathrm{SE}$, with $\mathrm{n}=$ arteries from 8-10 animals in for OZR; $\mathrm{n}=$ arteries from 6-8 animals for LZR. *; $p<0.05$ in the upper bound vs. LZR. $† ; p<0.05$ in the upper bound) vs. OZR. Please see text for details.

Figure 7. The correlation between vascular nitric oxide bioavailability and skeletal muscle microvessel density for LZR and OZR at 7-10 (black), 12-13 (blue), and 16-17 weeks of age (red), with the line of best fit through the aggregate data. Please see text for details. 
Table 1. Baseline characteristics of animal groups within the present study. Abbreviations: CAP (captopril), HDZ (hydralazine), MET (metformin), RGZ (rosiglitazone), TEM (TEMPOL), LNM (L-NAME), TLN (TEMPOL+L-NAME); * p<0.05 vs. LZR; † p<0.05 vs. OZR.

\begin{tabular}{|c|c|c|c|c|c|c|c|c|c|c|}
\hline & Weeks & LZR & OZR & OZR-CAP & OZR-HDZ & OZR-MET & OZR-RGZ & OZR-TEM & OZR-LNM & OZR-TLN \\
\hline Mass & $7-8$ & $148 \pm 7$ & $340 \pm 8^{*}$ & $338 \pm 9 *$ & $348 \pm 7 *$ & $331 \pm 8^{*}$ & $344 \pm 6^{*}$ & $351 \pm 6^{*}$ & $349 \pm 8^{*}$ & $346 \pm 10^{*}$ \\
\hline \multirow[t]{2}{*}{ (g) } & $12-13$ & $222 \pm 8$ & $458 \pm 11 *$ & $444 \pm 12 *$ & $446 \pm 10 *$ & $455 \pm 6^{*}$ & $448 \pm 8 *$ & $452 \pm 7 *$ & $455 \pm 10^{*}$ & $449 \pm 9 *$ \\
\hline & $16-17$ & $308 \pm 9$ & $688 \pm 14^{*}$ & $680 \pm 10 *$ & $679 \pm 12 *$ & $672 \pm 12 *$ & $681 \pm 7 *$ & $690 \pm 8 *$ & $684 \pm 11 *$ & $679 \pm 12 *$ \\
\hline MAP & $7-8$ & $104 \pm 4$ & $102 \pm 5$ & $98 \pm 4$ & $102 \pm 6$ & $97 \pm 6$ & $100 \pm 5$ & $102 \pm 6$ & $100 \pm 5$ & $99 \pm 6$ \\
\hline \multirow[t]{2}{*}{$(\mathrm{mmHg})$} & $12-13$ & $106 \pm 5$ & $112 \pm 6$ & $99 \pm 5$ & $100 \pm 6$ & $103 \pm 5$ & $105 \pm 4$ & $106 \pm 5$ & $128 \pm 5^{* \dagger}$ & $125 \pm 7 *$ \\
\hline & $16-17$ & $105 \pm 6$ & $134 \pm 7 *$ & $104 \pm 6 \dagger$ & $101 \pm 5 \dagger$ & $128 \pm 7^{*}$ & $120 \pm 5^{*}$ & $114 \pm 6 * \dagger$ & $151 \pm 6^{* \dagger}$ & $144 \pm 7 *$ \\
\hline Glucose & $7-8$ & $84 \pm 5$ & $88 \pm 5$ & $92 \pm 6$ & $91 \pm 6$ & $88 \pm 7$ & $92 \pm 6$ & $92 \pm 4$ & $92 \pm 4$ & $87 \pm 5$ \\
\hline \multirow[t]{2}{*}{ (mg/dl) } & $12-13$ & $92 \pm 5$ & $101 \pm 6$ & $105 \pm 6$ & $104 \pm 7$ & $101 \pm 6$ & $94 \pm 5$ & $99 \pm 5$ & $110 \pm 6^{*}$ & $109 \pm 7 *$ \\
\hline & $16-17$ & $94 \pm 4$ & $128 \pm 7 *$ & $111 \pm 9$ & $125 \pm 7 *$ & $106 \pm 5 \dagger$ & $99 \pm 6 \dagger$ & $119 \pm 6 *$ & $138 \pm 7 *$ & $135 \pm 10^{*}$ \\
\hline Insulin & $7-8$ & $0.9 \pm 0.2$ & $5.8 \pm 0.5^{*}$ & $5.5 \pm 0.4^{*}$ & $5.4 \pm 0.7^{*}$ & $5.4 \pm 0.5^{*}$ & $5.6 \pm 0.5^{*}$ & $5.2 \pm 0.4^{*}$ & $4.9 \pm 0.4^{*}$ & $5.4 \pm 0.3^{*}$ \\
\hline \multirow[t]{2}{*}{ (ng/ml) } & $12-13$ & $1.2 \pm 0.2$ & $6.9 \pm 0.6^{*}$ & $5.7 \pm 0.7^{*}$ & $6.6 \pm 0.5^{*}$ & $5.2 \pm 0.5^{* \dagger}$ & $4.7 \pm 0.4^{* \dagger}$ & $6.4 \pm 0.5^{*}$ & $7.4 \pm 0.5^{*}$ & $7.0 \pm 0.6^{*}$ \\
\hline & $16-17$ & $1.3 \pm 0.2$ & $8.4 \pm 0.5^{*}$ & $6.2 \pm 0.6^{* \dagger}$ & $8.0 \pm 0.7^{*}$ & $4.7 \pm 0.7^{* \dagger}$ & $4.2 \pm 0.5^{* \dagger}$ & $6.8 \pm 0.5^{* \dagger}$ & $9.0 \pm 0.6^{*}$ & $8.5 \pm 0.6^{*}$ \\
\hline N-tyrosine & $7-8$ & $8 \pm 3$ & $17 \pm 3^{*}$ & $16 \pm 5$ & $17 \pm 4$ & $15 \pm 5$ & $16 \pm 5$ & $17 \pm 4$ & $15 \pm 5$ & $17 \pm 4$ \\
\hline \multirow[t]{2}{*}{ (ng/ml) } & $12-13$ & $11 \pm 2$ & $31 \pm 5^{*}$ & $25 \pm 5^{*}$ & $29 \pm 4 *$ & $22 \pm 5$ & $21 \pm 6$ & $20 \pm 5$ & $47 \pm 6^{* \dagger}$ & $25 \pm 4 *$ \\
\hline & $16-17$ & $14 \pm 4$ & $54 \pm 8^{*}$ & $40 \pm 7 *$ & $47 \pm 7 *$ & $26 \pm 4 * \dagger$ & $24 \pm 5 \dagger$ & $22 \pm 6 \dagger$ & $72 \pm 7 * \dagger$ & $32 \pm 5^{*} \dagger$ \\
\hline
\end{tabular}




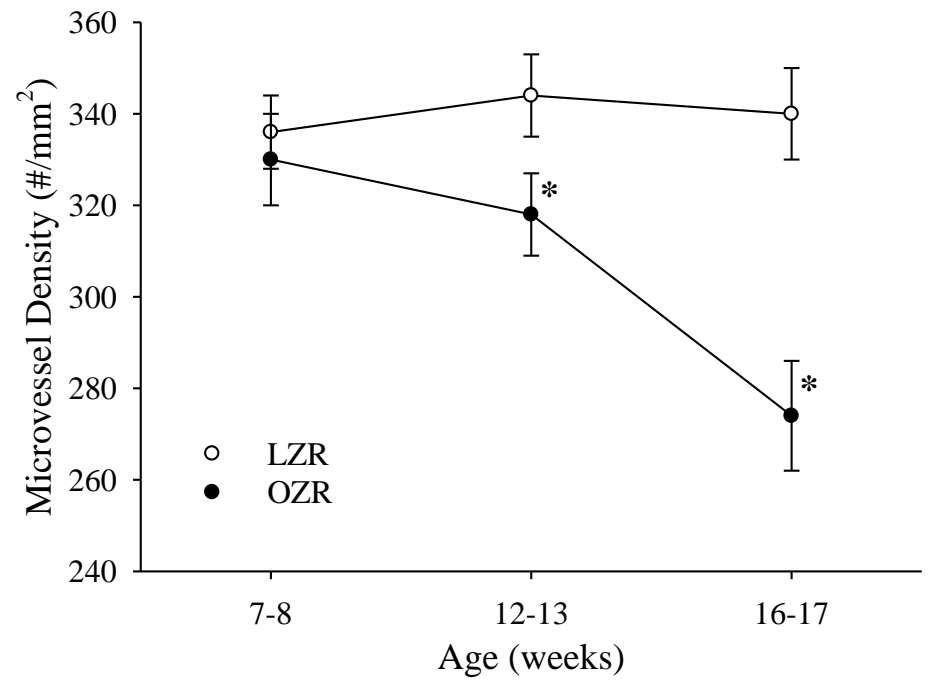

Figure 1.

Chantler et al. 


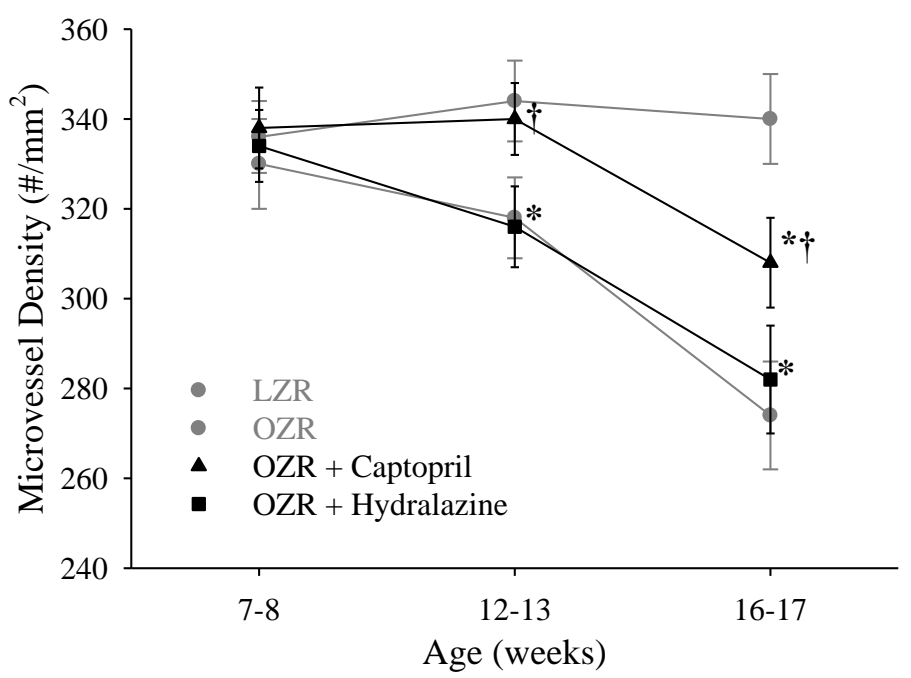

Figure 2.

Chantler et al. 


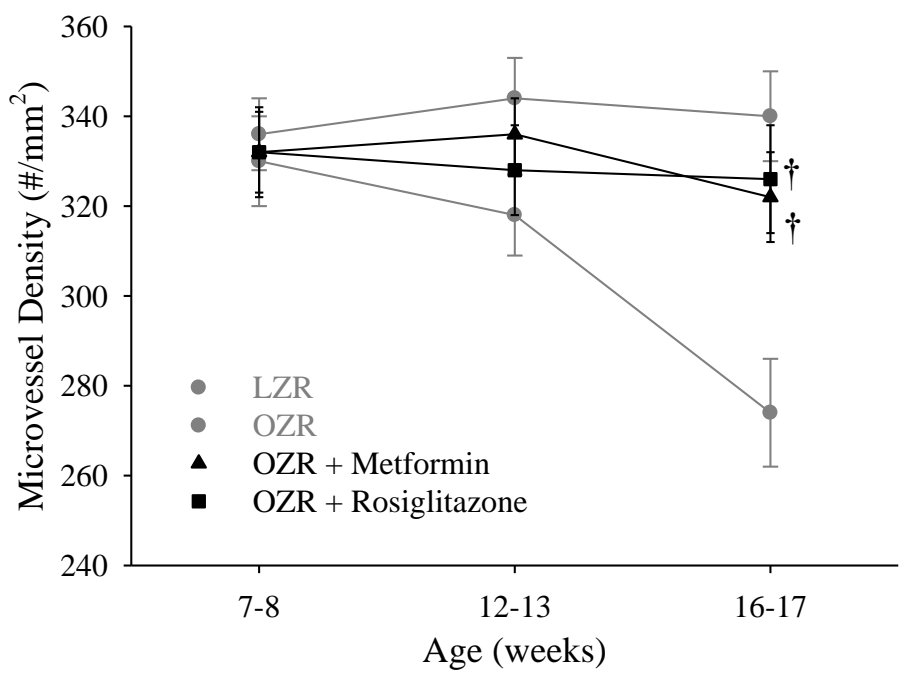

Figure 3.

Chantler et al. 


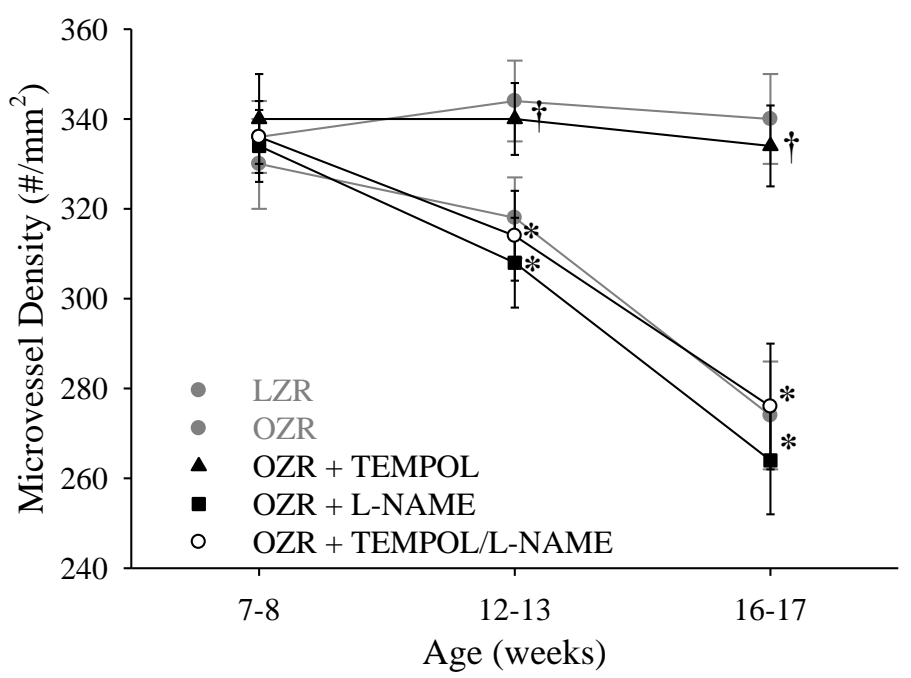

Figure 4.

Chantler et al. 


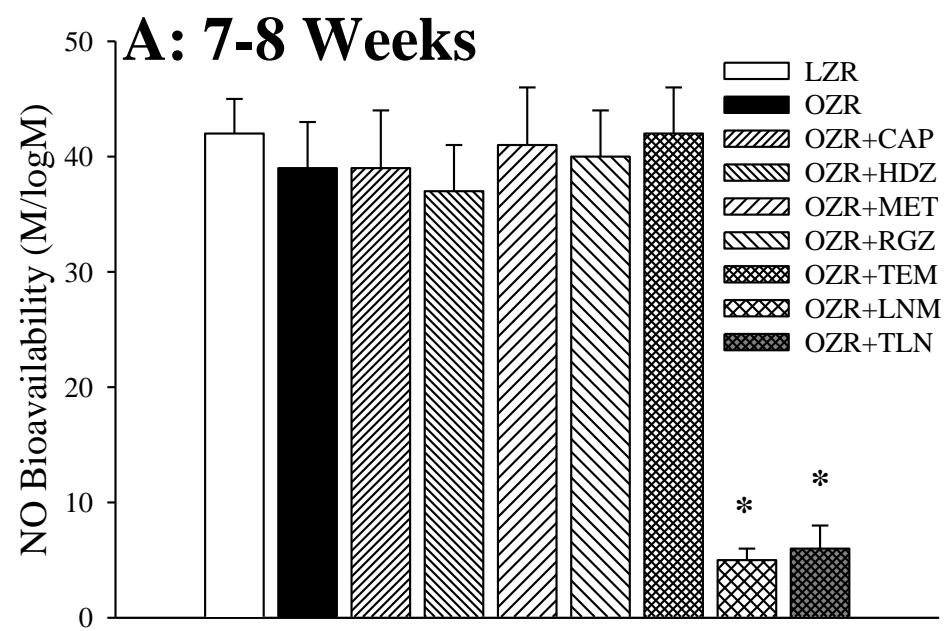

Experiment Group

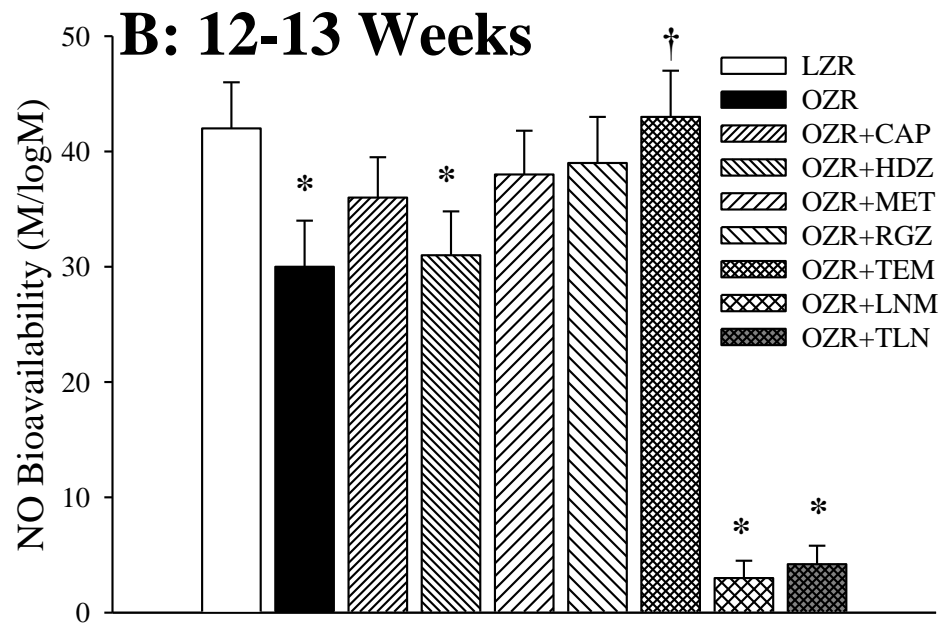

Experiment Group

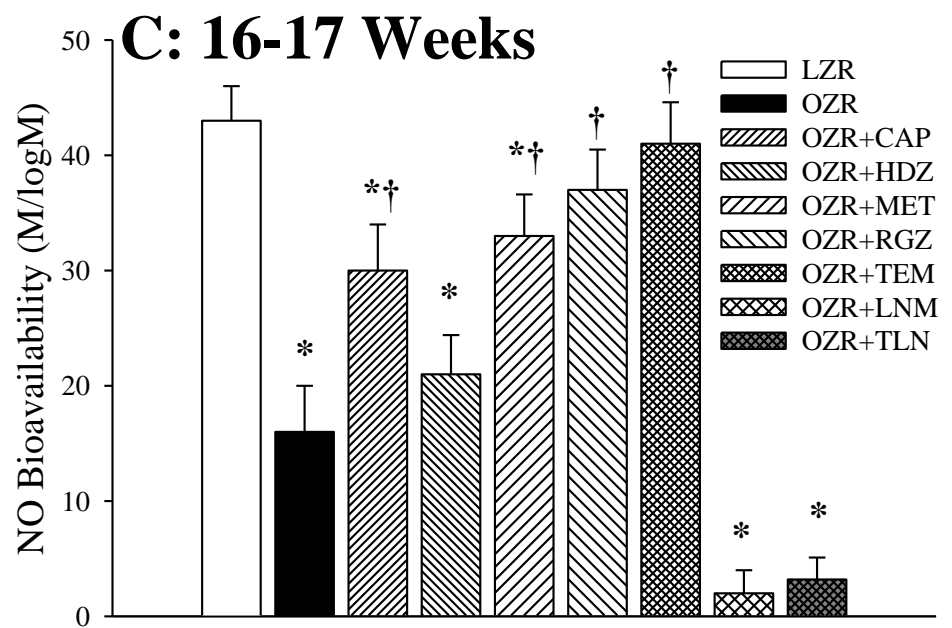

Experiment Group

Figure 5.

Chantler et al. 

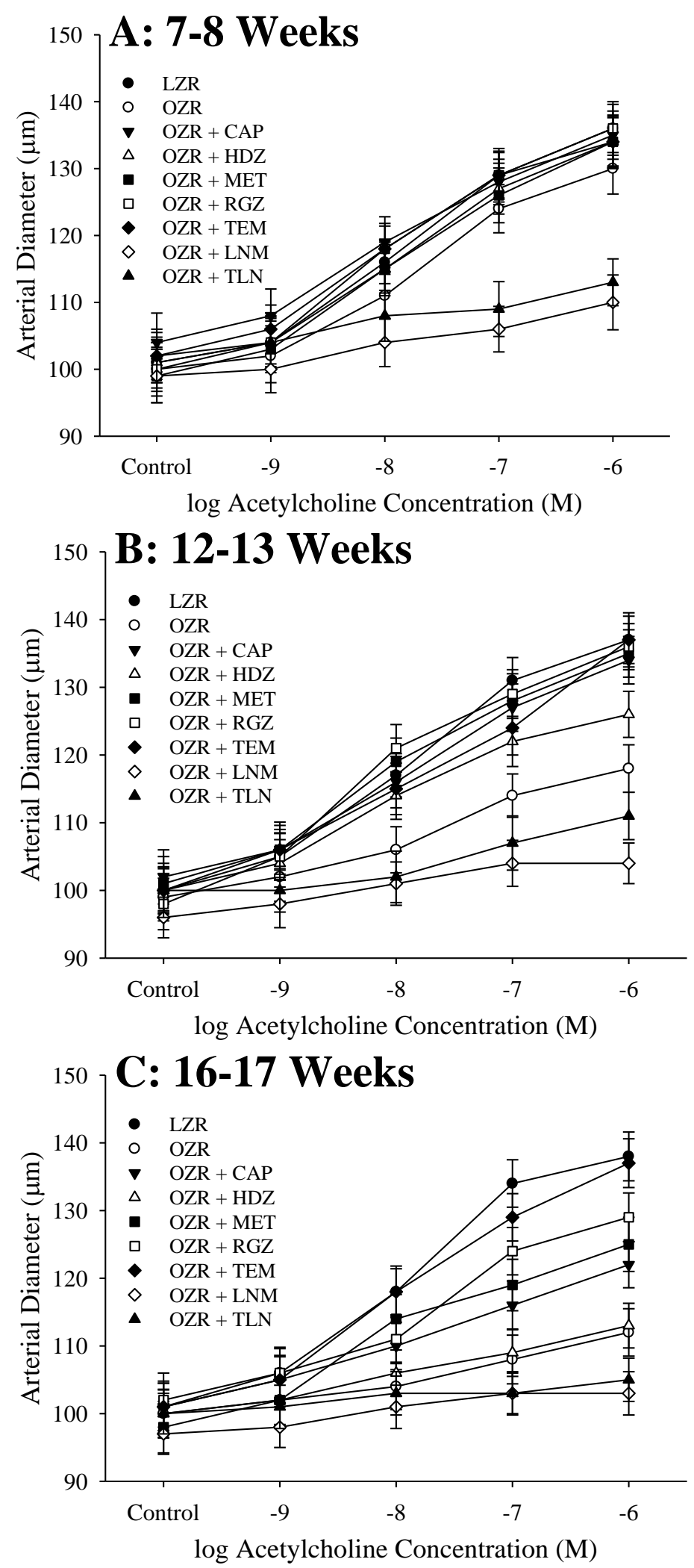

Figure 6.

Chantler et al. 


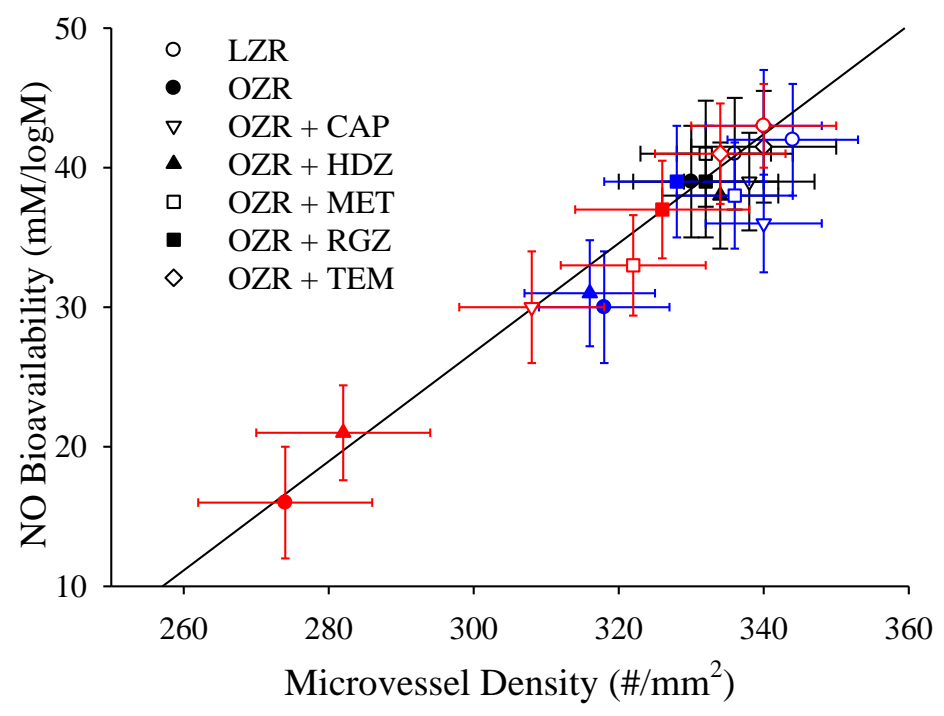

Figure 7.

Chantler et al. 


\section{Chapter 2: Conceptual Framework}

This chapter quantified the progressive decline in microvascular density in the frontal cortex of OZR which results from the development of MetSyn in this model. This study utilized clinically relevant antihypertensives and glycemic control agents to assess the contribution of underlying hypertension and hyperglycemia/insulin resistance on the development of cerebral cortical rarefaction. Microvascular density (MVD) was measured in LZR and OZR at 7-8 weeks (when symptoms of MetSyn first begin to appear), 12-13 weeks, and 16-17 weeks (consider full maturation of MetSyn vascular phenotype). A significant, progressive decline in cortical MVD in OZR was documented at each time point. OZR were then chronically treated from 7 weeks of age with an anti-hypertensive drug or a glycemic control agent, and evaluated for MVD at the same time points. Neither captopril nor hydralazine was able to prevent cerebral cortical rarefaction at 17 weeks, although captopril did delay onset and reduced rarefaction by almost half. In contrast, both metformin and rosiglitazone significantly improved MVD at 17 weeks, preventing the vast majority of rarefaction compared to untreated OZR.

The preliminary conclusion of these results was that management of hyperglycemia/insulin resistance is critical to preventing MetSyn-induced cortical rarefaction. However, treatment with TEMPOL completely prevented rarefaction in OZR, making it the most effective intervention, while treatment with L-NAME/TEMPOL resulted in the same progression of rarefaction as OZR control. These results are likely due to management of oxidant load in the cerebral circulation. A large body of literature has linked both type 1 and type 2 diabetes to cerebrovascular dysfunction, and identified reactive oxygen species (ROS) as the leading mechanisms. Additionally, angiotensin signaling in the brain is directly linked to generation of ROS, so treatment with an ACE inhibitor like captopril would be expected to reduce oxidative stress and thereby improve MVD. That TEMPOL was the most effective, and LNAME eliminated $100 \%$ of its benefit, supports this hypothesis. The measurements of NO 
bioavailability confirm this hypothesis, and a strong, linear relationship is evident between NO bioavailability and cerebral cortical MVD.

This chapter establishes that progression of MetSyn in ORZ leads to loss of NO bioavailability, with direct consequences for cerebral MVD. The next step was to investigate if impairments in cerebrovascular reactivity, and alterations in cerebral vessel wall mechanics, would also develop in OZR as a result of impaired NO bioavailability. The next chapter of this dissertation directly assessed this question using a similar experimental approach as this chapter, but expanding the scope of vascular outcomes. 


\section{CHAPTER 3:}

\section{METABOLIC SYNDROME IMPAIRS REACTIVITY AND WALL MECHANICS OF}

\section{CEREBRAL RESISTANCE ARTERIES IN OBESE ZUCKER RATS}

Steven D. Brooks ${ }^{1,6^{*}}$, Evan DeVallance ${ }^{2,6^{*}}$, Alexandre C. d'Audiffret ${ }^{3,5,6}$, Stephanie J. Frisbee ${ }^{5,6}$,

Lawrence E. Tabone ${ }^{3,5,6}$, Carl D. Shrader ${ }^{4,5,6}$, Jefferson C. Frisbee ${ }^{1,5,6}$ and Paul D. Chantler, $2,5,6$

Department of Physiology and Pharmacology ${ }^{1}$, Division of Exercise Physiology ${ }^{2}$, Department of Surgery $^{3}$, Department of Family Medicine ${ }^{4}$, Clinical and Translational Science Institute ${ }^{5}$, Center for Cardiovascular and Respiratory Sciences ${ }^{6}$; West Virginia University Health Sciences Center, Morgantown, WV

* S.D. Brooks and E. DeVallance contributed equally toward this work.

Running Head: Metabolic syndrome and cerebral vascular function

Send Correspondence to:

Jefferson C. Frisbee, Ph.D.

Department of Physiology and Pharmacology

Center for Cardiovascular and Respiratory Sciences

Robert C. Byrd Health Sciences Center

PO Box 9227

West Virginia University School of Medicine

Morgantown, WV 26505

Phone: (304) 293-6527

Fax: 304-293-5513

Email: jefrisbee@hsc.wvu.edu

Published originally in AJP Heart and Circulatory Physiology

(C) 2015 The American Physiological Society

Publisher requires no permissions for reproduction in dissertation/thesis

Authorship Statement: SD Brooks contributed to this manuscript in terms of original idea, data collection, data analysis, primary synthesis of manuscript, and manuscript review. 


\section{ABSTRACT}

The metabolic syndrome (MetS) is highly prevalent in the North American population, and is associated with increased risk for development of cerebrovascular disease. This study determined the structural and functional changes to the middle cerebral arteries (MCA) during the progression of MetS, and the effects of chronic pharmacological interventions on mitigating vascular alterations in obese Zucker rats (OZR), a translationally-relevant model of MetS. The reactivity and wall mechanics of ex vivo pressurized MCA from lean Zucker rats (LZR) and OZR was determined at 7-8, 12-13 and 16-17 weeks of age under control conditions and following chronic treatment with pharmacological agents targeting specific systemic pathologies. With increasing age, control OZR demonstrated reduced nitric oxide bioavailability, impaired dilator reactivity (acetylcholine), elevated myogenic activation, structural narrowing and wall stiffening versus LZR. Anti-hypertensive therapy starting at 7-8 weeks of age (e.g., captopril or hydralazine) blunted the progression of arterial stiffening compared to OZR controls, while treatments that reduced inflammation and oxidative stress (e.g., atorvastatin, rosiglitazone, captopril) improved NO bioavailability and vascular reactivity compared to OZR controls with mixed impacts on structural remodeling. These data show the functional and structural cerebral adaptions that limit cerebrovascular blood flow in MetS patients, contributing to increased risk of cognitive decline, cerebral hypoperfusion, and ischemic stroke; however, these pathological adaptations could potentially be blunted if treated early in the progression of MetS.

Keywords: Metabolic Syndrome, Cerebral vascular remodeling, arterial stiffness, reactive oxygen species. 


\section{INTRODUCTION}

The metabolic syndrome (MetS), a clustering of metabolic abnormalities such as obesity, impaired glycemic control, atherogenic dyslipidemia, hypertension, with the additional contributing conditions of a pro-oxidant, -thrombotic and -inflammatory state, is prevalent in $\sim 56$ million US adults [1]. Correspondingly, MetS is associated with a 3-fold increased risk of cardiovascular mortality [1], a 50\% increased risk of stroke [2], and is a known risk factor for cognitive decline with aging [3]. It is therefore imperative that translationally relevant models of MetS be effectively interrogated to guide our understanding of the cerebral vascular adaptations that are associated with the development of MetS, its physiological, molecular and genomic mechanistic underpinnings, and the effectiveness of established and novel therapeutic agents in either blunting or ameliorating MetS-associated cerebrovascular dysfunction.

The obese Zucker rat (OZR; $f a / f a)$ represents a translationally relevant animal model for the MetS in humans with high utility for studying negative cerebrovascular outcomes as a result of the constellation of systemic pathologies [4]. We have previously reported that cerebral resistance arteries from OZR develop significant alterations to vascular reactivity [5], and have demonstrated a progressive rarefaction of cerebral cortical microvessels with the progression of MetS. Of relevance, recent data suggest that cerebral infarct size in OZR is greater than in agematched control lean Zucker rat (LZR) [6] following ischemic stroke, and that this disparity in infarct severity with MetS development could be blunted with improved control of the chronic hypertension that develops [7]. While these are compelling initial observations regarding the impact of MetS on outcomes subsequent to ischemic stroke and the potential for risk factor control to lessen stroke severity following an ischemic episode, further information is necessary 
to discern the role for altered cerebrovascular function as a contributing factor for compromised outcomes.

The MetS in OZR is embodied by a few consistent overarching characteristics. Among these are a global reduction in the bioavailability of vascular-derived nitric oxide (and the associated alterations to vascular reactivity that this entails), the genesis of a systemic proinflammatory condition, and a progressive remodeling of the resistance and exchange microvasculature at multiple levels of resolution, including a change in the mechanics of the vascular wall and a reduction in tissue capillarity that have the potential to elevate perfusion resistance and impair processes of mass transport and exchange. However, the temporal development of these adaptations to the cerebral circulation throughout the progression of the MetS is not well understood and our ability to blunt them with relevant interventions remains virtually unknown. As such, the purpose of the present study was to: 1) study OZR at key age ranges throughout the development of the MetS to gain a fuller understanding of the temporal development of cerebrovascular adaptation/dysfunction with the evolution of the systemic pathologies and 2) to determine the extent to which interventions against the major constituent pathologies of the MetS are effective at blunting the development of alterations to vascular reactivity and vascular wall mechanics in the middle cerebral arteries of OZR.

\section{MATERIALS AND METHODS}

Animals: Male LZR and OZR (Harlan) arrived at the West Virginia University Health Sciences Center at 6-7 weeks of age and, after one week of acclimation to the local environment, were placed into a specific protocol cohort for the subsequent 9-10 weeks. Unless otherwise stated, animals were fed standard chow and tap water ad libitum for all experiments. All rats were housed in the animal 
care facility at the WVU HSC, and all protocols received prior IACUC approval. At 7-8 weeks of age, LZR ( $n=6$; time control groups only) and OZR ( $n=6$ for each group, at each age) were randomly placed into one of the following treatments for the subsequent 9-10 weeks:

1. Time control (normal food and water ad libitum).

2. Anti-hypertensive treatment with captopril (angiotensin converting enzyme inhibitor; 60 mg•kg-1 $\bullet$ day $^{-1}$; drinking water) [8].

3. Anti-hypertensive treatment with hydralazine (systemic vasodilator; $50 \mathrm{mg} \bullet \mathrm{kg}^{-1} \bullet \mathrm{day}^{-1}$; drinking water) [8].

4. Anti-diabetic treatment with metformin (hepatic gluconeogenesis inhibitor; $300 \mathrm{mg}^{\circ} \mathrm{kg}^{-}$ ${ }^{1} \bullet$ day $^{-1}$; drinking water) $[8,9]$.

5. Anti-diabetic treatment with rosiglitazone (insulin sensitizing agent; $10 \mathrm{mg} \mathrm{kg}^{-1} \bullet \mathrm{day}^{-1}$; mixed with food) $[8,10]$.

6. Anti-dyslipidemia treatment with atorvastatin (HMG Co-A reductase inhibitor; $25 \mathrm{mg} \mathrm{kg}^{-}$ ${ }^{1} \bullet$ day $^{-1}$; mixed with food) [11].

7. Anti-dyslipidemia treatment with gemfibrozil (peroxisome proliferator-activated receptor

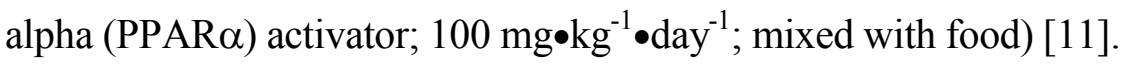

8. Anti-oxidant treatment with TEMPOL $\left(10^{-3} \mathrm{M}\right.$; drinking water) [12].

9. Nitric oxide synthase (NOS) inhibition with L-NG-Nitroarginine Methyl Ester (L-NAME, $10^{-4} \mathrm{M}$; drinking water) [12].

Following completion of the treatment period, each rat was anesthetized with injections of sodium pentobarbital (50 mg• $\mathrm{kg}^{-1}$ i.p.), and received tracheal intubation to facilitate maintenance of a patent airway. In all rats, a carotid artery and an external jugular vein were cannulated for determination of arterial pressure and for infusion of additional substances, respectively, as necessary (e.g., 
anesthetic, heparin, etc.). While under anesthetic, an aliquot of blood was drawn from the jugular vein to be used for the subsequent determination of plasma insulin, tumor necrosis factor- $\alpha$ (TNF- $\alpha$ ) and nitrotyrosine concentrations from each animal using commercially available kits (Millipore, Billerica, MA.

While deeply anesthetized, each rat was decapitated, after which the brain was removed from the skull case and placed in cold physiological salt solution (PSS; $4^{\circ} \mathrm{C}$ ). Subsequently, a middle cerebral artery (MCA) was dissected from its origin at the Circle of Willis. Each MCA was doubly cannulated within in a heated chamber $\left(37^{\circ} \mathrm{C}\right)$ that allowed the lumen and exterior of the vessel to be perfused and superfused, respectively, with PSS from separate reservoirs. The PSS was equilibrated with a $21 \% \mathrm{O}_{2}, 5 \% \mathrm{CO}_{2}$, and $74 \% \mathrm{~N}_{2}$ gas mixture and had the following composition (mM): $119 \mathrm{NaCl}, 4.7 \mathrm{KCl}, 1.17 \mathrm{MgSO}_{4}, 1.6 \mathrm{CaCl}_{2}, 1.18 \mathrm{NaH}_{2} \mathrm{PO}_{4}, 24 \mathrm{NaHCO}_{3}$, 0.026 EDTA, and 5.5 glucose. Any side branches were ligated using a single strand teased from 6-0 suture. Vessel diameter was measured using television microscopy and an on-screen video micrometer.

Measurements of Vascular Reactivity in Isolated MCA: Following cannulation, MCA extended to their in situ length and were equilibrated at $80 \%$ of the animal's mean arterial pressure $(88 \pm 4$ $\mathrm{mmHg}$ for LZR; $109 \pm 5 \mathrm{mmHg}$ for OZR) to approximate in vivo perfusion pressure [13]. Any vessel that did not demonstrate significant active tone at the equilibration pressure was discarded. Active tone at the equilibration pressure was calculated as $\left(\Delta \mathrm{D} / \mathrm{D}_{\max }\right) \bullet 100$, where $\Delta \mathrm{D}$ is the diameter increase from rest in response to $\mathrm{Ca}^{2+}$-free PSS, and $\mathrm{D}_{\max }$ is the maximum diameter measured at the equilibration pressure in $\mathrm{Ca}^{2+}$-free PSS. Active tone for vessels in the present study averaged $35 \pm 2 \%$ in LZR and $32 \pm 3 \%$ in OZR.

Following equilibration, the dilator reactivity of MCA was assessed in response to increasing 
concentrations of acetylcholine $\left(10^{-10} \mathrm{M}-10^{-6} \mathrm{M}\right)$. Vascular responses to acetylcholine (ACh) were also measured following acute incubation (45-60 minutes) with L-NAME $\left(10^{-4} \mathrm{M}\right)$ and TEMPOL $\left(10^{-4} \mathrm{M}\right)$, to assess the contributions of nitric oxide (NO) and oxidative stress, respectively, in modulating vascular reactivity.

Myogenic activation was assessed in MCA over the range of 40-140 $\mathrm{mmHg}$, in $20 \mathrm{mmHg}$ increments. Pressure was changed non-sequentially, and vessels were allowed 10 minutes to equilibrate at each pressure before arterial inner and outer diameters were recorded.

Following the experimental procedures for measuring ex vivo vascular reactivity, the perfusate and superfusate PSS were replaced with $\mathrm{Ca}^{2+}$-free PSS containing the metal ion chelators EDTA $(0.03 \mathrm{mM})$ and EGTA $(2.0 \mathrm{mM})$. Vessels were challenged with $10^{-7} \mathrm{M}$ serotonin until all active tone was lost. Subsequently, intralumenal pressure within the isolated vessel was altered, in $20 \mathrm{mmHg}$ increments, between $0 \mathrm{mmHg}$ and $160 \mathrm{mmHg}$. To ensure that a negative intralumenal pressure was not exerted on the vessel, $5 \mathrm{mmHg}$ was used as the " $0 \mathrm{mmHg}$ " intralumenal pressure point; all other values of intralumenal pressure were multiples of $20 \mathrm{mmHg}$ up to $160 \mathrm{mmHg}$. After 7 minutes at each intralumenal pressure, the inner and outer diameter of the passive MCA was determined.

Measurement of Vascular NO Bioavailability: From each rat, the abdominal aorta was removed and vascular NO production was assessed using amperometric sensors (World Precision Instruments, Sarasota, FL). Briefly, aortae were isolated, sectioned longitudinally, pinned in a silastic coated dish and superfused with warmed $\left(37^{\circ} \mathrm{C}\right)$ PSS equilibrated with $95 \% \mathrm{O}_{2}$ and $5 \%$ $\mathrm{CO}_{2}$. An NO sensor (ISO-NOPF 100) was placed in close apposition to the endothelial surface and a baseline level of current was obtained. Subsequently, increasing concentrations of methacholine $\left(10^{-10}-10^{-6} \mathrm{M}\right)$ were added to the bath and the changes in current were determined. 
To verify that responses represented NO release, these procedures were repeated following pretreatment of the aortic strip with L-NAME $\left(10^{-4} \mathrm{M}\right)$.

Data and Statistical Analyses: Mechanical responses following challenge with logarithmically increasing dosages of acetylcholine were fit with the three-parameter logistic equation:

$$
y=\min +\left[\frac{\max -\min }{1+10^{\log E D_{50}-x}}\right]
$$

where $y$ represents the vessel diameter, "min" and "max" represent the lower (minimum) and upper (maximum) bounds, respectively, of the change in diameter with agonist concentration, $x$ is the logarithm of the agonist concentration, and $\log \mathrm{ED}_{50}$ represents the logarithm of the agonist concentration $(x)$ where the response $(y)$ is halfway between the bounds. For the presentation of results, we have focused on the changes in the upper bounds as a representation of vessel reactivity, as lower bound remained consistent between all groups (defined as the pre-challenge diameter), and we did not determine a consistent or significant change to the $\operatorname{logED} \mathrm{D}_{50}$ values between treatment groups.

The myogenic activation for each experimental group was plotted as mean diameter at each intraluminal pressure and fitted with a linear regression $\left(y=\alpha_{0}+\beta x\right)$, where the slope coefficient $\beta$ represents the degree of myogenic activation ( $\delta$ diameter $/ \delta$ pressure). Increasingly negative values of $\beta$ therefore represent a greater degree of myogenic activation in response to changes in intralumenal pressure. A similar analysis was used to determine the nitric oxide bioavailability in response to increasing concentrations of methacholine, where $\beta$ represents the rate of change of nitric oxide released by the vessels in response to agonist challenge.

All calculations of passive arteriolar wall mechanics (used as indicators of structural alterations to the individual microvessel) are based on those used previously [14], with minor modification. 
Vessel wall thickness was calculated as:

$$
W T=\frac{(O D-I D)}{2}
$$

where WT represents wall thickness $(\mu \mathrm{m})$ and OD and ID represent arteriolar outer and inner diameter, respectively $(\mu \mathrm{m})$.

Incremental arteriolar distensibility $\left(\mathrm{DIST}_{\mathrm{INC}} ; \%\right.$ change in arteriolar diameter/mmHg) was calculated as:

$$
D I S T_{I N C}=\Delta I D /\left(I D \times \Delta P_{I L}\right) \times 100
$$

where $\Delta \mathrm{ID}$ represents the change in internal arteriolar diameter for each incremental change in intralumenal pressure $\left(\Delta \mathrm{P}_{\mathrm{IL}}\right)$.

For the calculation of circumferential stress, intralumenal pressure was converted from $\mathrm{mmHg}$ to $\mathrm{N} / \mathrm{m}^{2}$, where $1 \mathrm{mmHg}=1.334 \times 10^{2} \mathrm{~N} / \mathrm{m}^{2}$. Circumferential stress $(\sigma)$ was then calculated as:

$$
\sigma=\left(P_{I L} \times I D\right) /(2 W T)
$$

Circumferential strain $(\varepsilon)$ was calculated as:

$$
\varepsilon=\left(I D-I D_{5}\right) / I D_{5}
$$

where $\mathrm{ID}_{5}$ represents the internal arteriolar diameter at the lowest intralumenal pressure (i.e., 5 $\mathrm{mmHg}$. The stress versus strain relationship from each vessel was fit $\left(\mathrm{OLS}, \mathrm{r}^{2}>0.85\right)$ with the following exponential equation:

$$
\sigma=\sigma_{5} e^{\beta \varepsilon}
$$

where $\sigma_{5}$ represents circumferential stress at $\mathrm{ID}_{5}$ and $\beta$ is the slope coefficient describing arterial stiffness. Higher levels of $\beta$ are indicative of increasing arterial stiffness (i.e., requiring a greater 
degree of distending pressure to achieve a given level of wall deformation).

All data are presented as mean \pm SE. Differences in passive mechanical characteristics, slope coefficients describing vascular reactivity or NO bioavailability, or descriptive characteristics between LZR and OZR groups were assessed using analysis of variance (ANOVA), with StudentNewman-Keuls-test post-hoc, as appropriate. In all cases, $\mathrm{p}<0.05$ was taken to reflect statistical significance.

\section{RESULTS}

Table 1 presents body mass, mean arterial pressure, and plasma biomarker data for each of the animal groups in the present study at three time points: 7-8 weeks, 12-13 weeks, and 16-17 weeks of age. With increasing age, control OZR developed characteristic symptoms of MetS compared to LZR control, including elevated MAP, hyperglycemia and elevated plasma insulin. Chronic treatment of OZR with specific pharmacological agents improved the expected outcomes for each drug's targeted risk factors. Specifically, treatment with captopril or hydralazine abrogated the development of hypertension, treatment with metformin or rosiglitazone improved glycemic control, treatment with atorvastatin or gemfibrozil blunted the severity of the developing dyslipidemia in OZR, and chronic treatment with TEMPOL blunted the oxidant stress levels in OZR versus LZR. Conversely, chronic NOS inhibition by treatment with L-NAME accelerated the development of hypertension and resulted in an increased level of oxidant stress.

Figure 1 presents mechanical properties for arterial inner diameter (ID) and circumferential stress vs. strain relationships, reactivity data for vasodilation to ACh and myogenic activation for OZR and LZR control at all three age ranges points, as well as arterial NO bioavailability under LNAME treated, TEMPOL treated, and control conditions. By 16-17 weeks, both LZR and OZR 
demonstrated a significant decrease in inner diameter at elevated intraluminal pressure as compared to their values at 7-8 weeks, with OZR also exhibiting a decreased inner diameter by 12-13 weeks of age (Panel A), with a significant left-shifting of the circumferential stress vs. strain relationship at both age groups in OZR as compared to values from the young age group (Panel B). LZR exhibited a robust vasodilation in response to $\mathrm{ACh}$ at each age group, while reactivity to $\mathrm{ACh}$ was steadily reduced with increasing age (Panel C). The slope of myogenic activation did not change between age groups in LZR, while the $\beta$ coefficients became increasingly negative with increasing age in OZR (Panel D). NO bioavailability did not change with age in LZR, while it decreased steadily in OZR at both 12-13 weeks and again at 16-17 weeks (Panel E). Treatment with TEMPOL did not significantly alter NO bioavailability in LZR, but did improve NO bioavailability in OZR at both 12-13 weeks and 16-17 weeks. At all ages, in both LZR and OZR, treatment with L-NAME was able to nearly eliminate NO bioavailability.

The impact of chronic treatment of OZR with the anti-hypertensive agents captopril or hydralazine on arterial wall mechanics, reactivity, and NO bioavailability at 12-13 and 16-17 weeks are presented in Figure 2. Generally, chronic treatment with either agent blunted remodeling of the MCA wall in OZR, blunting changes to the MCA inner diameter (Panel A) and the stress vs strain relationship (Panel B). The effect on vascular reactivity was heterogeneous, as captopril treatment better maintained dilator reactivity to ACh versus OZR control, while the hydralazine-treated cohort showed only minimal improvements (Panel C). Both anti-hypertensive treatments reduced the $\beta$ coefficient of myogenic activation to levels comparable to LZR (Panel D). Captopril significantly improved NO bioavailability at 12-13 weeks and 16-17 weeks, while hydralazine had no effect at 12-13 weeks and only a modest improvement in NO bioavailability at 16-17 weeks. 
Data describing impact of chronic treatment of OZR with the anti-diabetic treatments rosiglitazone or metformin on vessel wall mechanics, vascular reactivity, and NO bioavailability at 12-13 and 16-17 weeks are presented in Figure 3. While neither rosiglitazone nor metformin resulted in significant alterations in MCA inner diameter at 12-13 weeks, both showed small but significant improvements at 16-17 weeks of age (Panel A). Treatment with rosiglitazone reduced the changes to the stress vs strain $\beta$ coefficient primarily at $16-17$ weeks; metformin had only a slight effect at 16-17 weeks (Panel B). Both metformin and rosiglitazone exhibited moderately improved reactivity to ACh at 16-17 weeks (Panel C), and both drugs were also able to improve the $\beta$ coefficient of the myogenic response at 16-17 weeks (Panel D). While both metformin and rosiglitazone significantly improved NO bioavailability at 12-13 weeks, only rosiglitazone remained effective at 16-17 weeks of age (Panel E)

The impact of chronic treatment of OZR with the anti-dyslipidemia treatments atorvastatin or gemfibrozil on arterial wall mechanics, reactivity, and NO bioavailability at 12-13 and 16-17 weeks are presented in Figure 4. As with the glycemic control agents described above, chronic treatment with the dyslipidemia agents did not significantly alter the inner diameter of the MCA from OZR in response to elevated intraluminal pressure compared to LZR control (Panel 4A). However, atorvastatin was able to significantly reduce the stress vs strain $\beta$ coefficient at both 12-13 weeks and 16-17, while gemfibrozil had no effect at the younger age and only a small impact at 1617 weeks (Panel 4B). Similarly, atorvastatin was highly effective at improving reactivity to ACh (Panel 4C) and NO bioavailability (Panel 4E) at both ages, while gemfibrozil had no effect on either metric at any age. Both agents were able to significantly improve the $\beta$ coefficient of the myogenic response at 12-13 weeks and 16-17 weeks (Panel 4D). 
Figure 5 presents the impact of chronic treatment with the anti-oxidant agent TEMPOL, and of chronic treatment with the competitive NOS-inhibitor L-NAME, on arterial wall mechanics, reactivity, and NO bioavailability in OZR at 12-13 and 16-17 weeks. TEMPOL, which targets the increases in oxidant stress that accompany the onset of metabolic syndrome in OZR, reduced the narrowing of the MCA inner diameter to levels matching LZR control (Panel A), and showed the greatest reduction in stress vs strain $\beta$ coefficients of all treatment groups (Panel B). In contrast, chronic treatment with L-NAME exacerbated arterial remodeling, and had no effect on stress vs strain $\beta$ coefficient at either age (Fig. 5 B). Chronic treatment with TEMPOL resulted in the most robust dilation to ACh of any treatment group, while L-NAME nearly abolished all responses to ACh across both strains and all ages (Panel C). While TEMPOL improved the $\beta$ coefficient of the myogenic response of MCA from OZR at both older ages, L-NAME negatively increased the $\beta$ coefficient, as was in fact the only treatment to do so (Panel D). In agreement with the reactivity data, TEMPOL significantly improved NO bioavailability from OZR at both older ages, while LNAME severely reduced NO bioavailability to levels even lower than OZR control (Panel E5E).

The relationship between NO bioavailability, $\beta$ coefficients from the stress vs. strain curves, and the upper bound of ACh-induced vasodilation for each animals at each of the three age ranges are displayed in Figure 6. At 7-8 weeks, OZR has not yet fully developed the metabolic syndrome pathologies, which is reflected by the clustering of data points into one small area (Panel A). By 1213 weeks, OZR have developed MetS and demonstrate increased stress vs strain $\beta$ coefficients along with reduced NO bioavailability and decreased ACh upper bounds (Panel B). In panel B, atorvastatin and TEMPOL-the treatments which best preserved NO bioavailability and ACh upper bound, while mitigating increases in stress vs strain $\beta$ coefficients with increasing severity of MetS. In contrast, gemfibrozil and metformin, which were overall two of the least effective treatments, 
already demonstrated significant movement in the relationship at only 12-13 weeks of age. This trend continues at 16-17 weeks, where the OZR controls are now extremely divergent from LZR, representing significantly increased stress-strain $\beta$ coefficients and poor NO bioavailability/vascular reactivity. Gemfibrozil and metformin have similarly dropped as well, as has hydralazine, suggesting that while hydralazine was able to delay the deleterious effects of MetS at 12-13 weeks, it is no longer able to do so by $16-17$ weeks. Atorvastatin and TEMPOL still remain towards the upper right quadrant, while captopril and rosiglitazone populate the middle quadrants (Fig. 6C).

Figure 7 plots the stress vs. strain $\beta$ coefficients against mean arterial pressures at 16-17 weeks. The four treatments which most effectively controlled hypertension in OZR- TEMPOL, atorvastatin, hydralazine, and captopril- also exhibited $\beta$ coefficients in the stress vs. strain curve that were most similar to that for LZR control MCA. These treatment groups with the lowest $\beta$ coefficients, all had a final MAP at 17 weeks that was not significantly different from LZR control. The other 4 treatments all had $\beta$ coefficients $>2.2$ and had significantly higher MAP compared to LZR control.

\section{DISCUSSION}

In the absence of intervention, OZR experience a progressive decline in vascular structure, mechanics, and reactivity in the MCA, associated with the progressing MetS state. However, changes in mechanical properties (i.e. distensibility) precede that of changes in lumen diameter and wall thickness in MetS. At 7-8 weeks of age, OZR are significantly heavier and have elevated levels of TNF- $\alpha$ and insulin; but show minimal changes to markers of oxidant stress and blood pressure, and minor changes to vascular reactivity. The MCAs of OZR and LZR remain dimensionally similar at 7-8 weeks, but substantial arterial stiffening is already present as 
reflected by the left-shifted stress-strain curve and increased $\beta$ coefficient. At 12-13 weeks of age, inflammation and insulin levels have risen further, while substantial oxidative damage has accumulated, along with increased blood pressure. This is reflected in further mechanical stiffening of the MCA, likely through increase deposition, and crosslinking of collagen, plus fragmentation of elastin shift load to collagen at lower pressures, thereby making the vessel less distensible [15]. Oxidative and inflammatory damage have negatively impacted the endothelium and reduce NO bioavailability, resulting in impaired dilator reactivity, which is alleviated by acute treatment with the antioxidant TEMPOL. Additionally, eutrophic structural remodeling (lumen and outer diameters decrease with little change in wall thickness) of the MCA is present in OZR, and stiffness of the MCA continues to worsen as measured by increased $\beta$ coefficients of the stress-strain relationship. By 16-17 weeks, as inflammation, oxidative stress, and blood pressure have all increased with age and severity of the MetS, the degree of arterial dysfunction, remodeling, and stiffening continue to worsen. It is possible that the progressive loss of vascular wall distensibility may have contributed to the reduction of dilator reactivity between LZR and OZR and across the different interventions. However, the extent to which wall distensibility contributed to impaired reactivity is difficult to assess owing to the concurrent impairment of the mechanisms of dilator reactivity (i.e., loss of NO bioavailability).

The increase in arterial stiffness was closely associated with the onset of hypertension, indicative of the well-established relationship between elevated arterial pressure and vascular remodeling[16]. In the present study, the use of atorvastatin, TEMPOL, captopril, or hydralazine blunted the onset of hypertension, and were most effective at mitigating the increased arterial stiffness. While atorvastatin, tempol, and captopril reduced inflammation and oxidative stress, as well as blood pressure, hydralazine had minimal effects on inflammation and oxidative stress, 
suggesting that efficacy for controlling arterial stiffness may be primarily related to the antihypertensive actions of atorvastatin, TEMPOL or captopril rather than any pleiotropic effect. Of particular relevance, clinical observations in hypertensive human subjects have shown that chronic treatment with ACE inhibitors can reduce the severity of large artery stiffness with prolonged hypertension[17]. However, it should be noted that a limitation of the present study is that we do not have chronic measurements of arterial pressure under the different conditions of the study and only present data from the three age ranges.

While the relationship between the onset of MetS in OZR and the presence of arterial dysfunction has been well established, this study found that four of the interventions (atorvastatin, TEMPOL, captopril, and rosiglitazone) improved NO bioavailability and endothelium-dependent dilation in 16-17 week old OZR. As shown in Table 1, these pharmacological interventions reduces levels of TNF- $\alpha$, MCP-1, and nitrotyrosine, suggesting that treatments conferring both anti-inflammatory and anti-oxidant benefits are most effective at improving NO bioavailability and arterial function in OZR. Atorvastatin for example, has welldefined roles in improving endothelial function, increasing NO bioavailability, reducing MAP, and anti-inflammatory/anti-oxidant effects in addition to its cholesterol-lowering mechanism [18, 19].

In order to assess the effects of impaired glycemic control on arterial remodeling and dysfunction, OZR were treated with metformin or rosiglitazone. Both drugs improved insulin resistance, with minimal effects on arterial pressure. Although metformin had little effect on the remodeling or stiffness of the MCA in OZR, rosiglitazone did blunt both structural narrowing and the loss of MCA elasticity. Additionally, rosiglitazone was more effective at improving dilator responses to $\mathrm{ACh}$ and arterial $\mathrm{NO}$ bioavailability than metformin. The divergence in the 
effectiveness of these anti-diabetic agents for MCA structure and function may lie within the additional effects of rosiglitazone on improving endothelial function, inflammation, remodeling and blood pressure, that do not appear to be as robust with metformin[20, 21]. It is possible that metformin may be more effective at preventing remodeling at later stages of insulin resistance/type II diabetes after prolonged treatment, when it shows greatest cardiovascular risk reduction clinically[22], although this speculation is beyond the scope of the present study.

Dyslipidemia is common in MetS, and statins are the most popular front line pharmacological agents in treatment of this condition. As stated above, atorvastatin has numerous pleotropic effects in the context of cardiovascular disease, such as improving endothelial function via increasing NO bioavailability, and boosting the innate anti-oxidant system [23]. In this study, atorvastatin imparted significant improvements to arterial reactivity, and coupled a decrease in oxidative load with a robust anti-inflammatory effect. These effects corresponded with limited remodeling of the MCA and reduced arterial stiffness compared to OZR control. These observations support those from previous studies demonstrating that atorvastatin treatment improved endothelial function in diabetes and cardiomyopathy [24, 25]. In contrast, gemfibrozil, a peroxisome proliferator-activated receptor agonist with potent triglyceride lowering actions [26], had little effect on arterial stiffness, distensibility, or reactivity, especially at 16-17 weeks of age in OZR. This disparity provides additional support for the concept that the beneficial effects of atorvastatin stem not necessarily from management of cholesterol, but rather from its pleotropic cardiovascular benefits [11, 27]. Similarly, gemfibrozil alone or in combination with niacin did not significantly improve endothelial function in patients with normal or elevated cholesterol levels [28]. 
TEMPOL was included in this study as a positive treatment standard for treating chronic low-level inflammation and oxidative stress associated with MetS [29]. Oxidative stress is known to play an important role in the pathogenesis of vascular dysfunction [30]. Not only does a pro-oxidative stress (ROS) environment impact the NO pathway and its regulation of arterial remodeling, but ROS can independently interact with the components of the perivascular matrix and can drive collagen cross-linking, collagen deposition, and the fracturing of elastin [31]. Previous evidence suggests that eutrophic inward remodeling was dependent on ROS activation of specific matrix metalloproteinases (that degrade and reorganize the extracellular matrix of the vessel wall) [32]. Importantly, TEMPOL was highly effective at lowering nitrotyrosine, TNF- $\alpha$, and MCP-1 concentrations in the OZR (Table 1), in addition to improving arterial reactivity, reducing stiffness, and restoring NO bioavailability. It is important to note that all measurements of NO bioavailability were made using conduit artery segments (aorta), and not harvested MCA. As such, results should be interpreted appropriately.

The observation of increased myogenic properties of ex vivo MCA in the present study is consistent with previous observations and across multiple tissues and organs from OZR and other similar models of MetS [17, 22, 24, 43, 49]. While mechanistic contributors to increased pressure-induced constriction remain to be fully elucidated, it does appear that the progressive dysfunction of the vascular endothelium may act to remove a "buffer" on the robustness of the constrictor response and contribute to the enhanced myogenic properties. It is unclear if this reflects the loss of vascular NO bioavailability or a broader condition involving other mechanisms of endothelial dysfunction and alterations in vascular smooth muscle responses per se. However, on the basis of the results from the interventional procedures in the present study, this appears to be more complicated than a simple "recovery of endothelial function," as 
correlations between improvements in vascular NO bioavailability (Figs. 2E, 3E, 4E, and 5E) were not perfectly associated with normalization of myogenic constriction in treated OZR (Figs. $2 \mathrm{D}, 3 \mathrm{D}, 4 \mathrm{D}$, and $5 \mathrm{D})$. This suggests that there may be alterations in vascular smooth muscle itself and responses to intraluminal pressure with the development of the MetS [23, 37, 44] that are not as readily correctable by improved endothelial function alone.

Translational Relevance: In addition to the work from Stepp's group demonstrating increase stroke infarct severity in OZR [39, 40]. Given that cognitive impairments and vascular dementia have been linked to impairments of blood flow regulation and cerebral perfusion control, the cerebrovascular adaptations occurring in MetS may represent key contributors to multiple cerebral pathologies[33-35]. We have previously reported a decrease in cerebral cortical microvascular density associated with MetS, and suggested that this rarefaction may contribute to either accumulated stroke risk or poor health outcomes in afflicted individuals[36]. In the present study, we have further added to the potential mechanisms by which MetS may contribute to impaired cerebral perfusion and increased risk of ischemic stroke by describing cerebrovascular dysfunction along with reduced cerebral arterial wall distensibility. In combination with microvessel rarefaction, these additional alterations may serve as physiological contributors to significant impairments in cerebral blood flow regulation and perfusion, which have been linked to cognitive decline with aging. Indeed, provocative recent work by Tucsek et al[37], who found that aging in combination with high-fat diet induced obesity in mice led to an exacerbation of cerebrovascular rarefaction and neurovascular uncoupling previously observed in obese mice and associated cognitive impairments, may provide additional insight. MetSassociated cerebrovascular changes can also lead to elevated risk for ischemic stroke in MetS, as well as worsened stroke outcomes in patients suffering from a MetS as a pre-existing condition 
through compromised processes of mass transport and exchange, leading to an increased risk for areas of ischemic damage.

Previous research has demonstrated that MetS in OZR is associated with cognitive impairments in memory testing through hippocampal-dependent pathways [38], and similar impairments in cognitive function and memory recall have been observed in other rat strains fed high-fat Western diets [39] [40, 41]. Furthermore, rats experiencing mild disruption of cerebral blood flow through a transient ischemia model developed cognitive decline and memory impairments ([42]), while rats exposed to elevated oxidative stress developed cognitive deficits as a result of direct implications in the hippocampus and frontal cortex ([43]). Taken together, these findings suggest that the cerebral vasculopathies resulting from MetS and its underlying conditions can contribute to impairments in cerebral blood flow regulation and to an ensuing cognitive decline. Similarly, cognitive impairments in aged rats have been directly linked to regional changes in brain glucose availability and metabolism ([44]). With a growing body of clinical and epidemiological literature implicating MetS and other cardiovascular disease risk factors with the development of cognitive impairment [3][45][46], the structural and functional impairments in the cerebral vasculature highlighted in this study may provide novel insight for preventing or treatment of cognitive impairments in at-risk patients.

Thus, early recognition of MetS and therapeutic intervention targeted at protecting the cerebral vasculature is critical. Although blood pressure control alone was effective in dampening arterial stiffening, in the present study we found atorvastatin to be the most effective treatment for ameliorating cerebrovascular dysfunction. It is noteworthy that there is substantial evidence to support the beneficial effect of statins on limiting infarct size as a result of stroke and improving recovery[47]. It has also been demonstrated that the use of statins during 
hospitalization is associated with improved survival and a better discharge disposition among patients following ischemic stroke [48]. This cumulative evidence would suggest that early treatment with 3-hydroxy-3-methylglutarylcoenzyme A reductase inhibitors to utilize their pleiotropic effects on blood pressure, inflammation, and oxidative stress might be a useful clinical tool for combating MetS-related cerebrovascular disorders.

\section{Acknowledgements:}

The authors would like to thank Ms. Milinda James for her expert technical assistance. Additionally, we also acknowledge the support provided through Center for Cardiovascular and Respiratory Sciences and the Clinical and Translational Sciences Institute at the West Virginia University Health Sciences Center.

\section{Grants}

This study was supported by the American Heart Association (IRG 14330015, PRE 16850005, EIA 0740129N), and the National Institutes of Health (1P20 GM109098, RR 2865AR; P20 RR

016477). Research reported in this publication was supported by the National Institute of General Medical Sciences of the National Institutes of Health under Award Number U54GM104942.

\section{Disclaimer}

The content is solely the responsibility of the authors and does not necessarily represent the official views of the National Institutes of Health. 


\section{LITERATURE CITED:}

1. Grundy, S.M., et al., Diagnosis and management of the metabolic syndrome: An American Heart Association/National Heart, Lung, and Blood Institute scientific statement. Current Opinion in Cardiology, 2006. 21(1): p. 1-6.

2. Boden-Albala, B., et al., Metabolic Syndrome and Ischemic Stroke Risk: Northern Manhattan Study. Stroke, 2008. 39(1): p. 30-35.

3. Panza, F., et al., Metabolic syndrome and cognitive impairment: current epidemiology and possible underlying mechanisms. J Alzheimers Dis, 2010. 21(3): p. 691-724.

4. Aleixandre de Artinano, A. and M. Miguel Castro, Experimental rat models to study the metabolic syndrome. Br J Nutr, 2009. 102(9): p. 1246-53.

5. Butcher, J.T., et al., Differential impact of dilator stimuli on increased myogenic activation of cerebral and skeletal muscle resistance arterioles in obese zucker rats. Microcirculation, 2013. 20(7): p. 579-89.

6. Osmond, J.M., et al., Obesity Increases Blood Pressure, Cerebral Vascular Remodeling, and Severity of Stroke in the Zucker Rat. Hypertension, 2009. 53(2): p. 381-386.

7. Osmond, J.M., J.D. Mintz, and D.W. Stepp, Preventing increased blood pressure in the obese Zucker rat improves severity of stroke. American Journal of Physiology - Heart and Circulatory Physiology, 2010. 299(1): p. H55-H61.

8. Goodman and Gilman's The Pharmacological Basis of Therapeutics. 11th Edition ed. 2005: McGraw-Hill Professional.

9. Wessels, B., et al., Metformin impairs mitochondrial function in skeletal muscle of both lean and diabetic rats in a dose-dependent manner. PLoS One, 2014. 9(6): p. e100525.

10. Sardone, L.D., et al., Effect of rosiglitazone on bone quality in a rat model of insulin resistance and osteoporosis. Diabetes, 2011. 60(12): p. 3271-8.

11. Goodwill, A.G., et al., Impact of chronic anticholesterol therapy on development of microvascular rarefaction in the metabolic syndrome. Microcirculation, 2009. 16(8): p. 667-84. 12. Frisbee, J.C., Reduced nitric oxide bioavailability contributes to skeletal muscle microvessel rarefaction in the metabolic syndrome. Am J Physiol Regul Integr Comp Physiol, 2005. 289(2): p. R307-R316.

13. Lombard, J.H., et al., Electrical and mechanical responses of rat middle cerebral arteries to reduced PO2 and prostacyclin. Am J Physiol, 1999. 276(2 Pt 2): p. H509-16. 
14. Baumbach, G.L. and M.A. Hajdu, Mechanics and composition of cerebral arterioles in renal and spontaneously hypertensive rats. Hypertension, 1993. 21(6 Pt 1): p. 816-26.

15. Lakatta, E.G. and D. Levy, Arterial and cardiac aging: major shareholders in cardiovascular disease enterprises: Part I: aging arteries: a "set up" for vascular disease. Circulation, 2003. 107(1): p. 139-46.

16. Payne, R.A., I.B. Wilkinson, and D.J. Webb, Arterial stiffness and hypertension: emerging concepts. Hypertension, 2010. 55(1): p. 9-14.

17. Mahmud, A. and J. Feely, Reduction in arterial stiffness with angiotensin II antagonist is comparable with and additive to ACE inhibition. Am J Hypertens, 2002. 15(4 Pt 1): p. 321-5.

18. Davignon, J., Beneficial cardiovascular pleiotropic effects of statins. Circulation, 2004. 109(23 Suppl 1): p. III39-43.

19. Kuklinska, A.M., et al., Influence of atorvastatin on blood pressure control in treated hypertensive, normolipemic patients - An open, pilot study. Blood Press, 2010. 19(4): p. 260-6.

20. Kelly, A.S., et al., Rosiglitazone improves endothelial function and inflammation but not asymmetric dimethylarginine or oxidative stress in patients with type 2 diabetes mellitus. Vasc Med, 2007. 12(4): p. 311-8.

21. Kiyici, S., et al., Effect of rosiglitazone, metformin and medical nutrition treatment on arterial stiffness, serum MMP-9 and MCP-1 levels in drug naive type 2 diabetic patients. Diabetes Res Clin Pract, 2009. 86(1): p. 44-50.

22. Lamanna, C., et al., Effect of metformin on cardiovascular events and mortality: a metaanalysis of randomized clinical trials. Diabetes Obes Metab, 2011. 13(3): p. 221-8.

23. Crisby, M., et al., Pravastatin treatment increases collagen content and decreases lipid content, inflammation, metalloproteinases, and cell death in human carotid plaques: implications for plaque stabilization. Circulation, 2001. 103(7): p. 926-33.

24. Economides, P.A., et al., The effects of atorvastatin on endothelial function in diabetic patients and subjects at risk for type 2 diabetes. J Clin Endocrinol Metab, 2004. 89(2): p. 740-7.

25. Liu, M., et al., Atorvastatin improves endothelial function and cardiac performance in patients with dilated cardiomyopathy: the role of inflammation. Cardiovasc Drugs Ther, 2009. 23(5): p. 369-76. 
26. Wagner, A.M., et al., Efficacy of atorvastatin and gemfibrozil, alone and in low dose combination, in the treatment of diabetic dyslipidemia. J Clin Endocrinol Metab, 2003. 88(7): p. 3212-7.

27. Chironi, G., et al., Differential associations of statin and fibrate treatment with carotid arterial remodeling. Am J Hypertens, 2005. 18(11): p. 1476-81.

28. Andrews, T.C., et al., Effect of gemfibrozil +/- niacin +/- cholestyramine on endothelial function in patients with serum low-density lipoprotein cholesterol levels $<160 \mathrm{mg} / \mathrm{dl}$ and highdensity lipoprotein cholesterol levels <40 mg/dl. Am J Cardiol, 1997. 80(7): p. 831-5.

29. Ford, E.S., The metabolic syndrome and $C$-reactive protein, fibrinogen, and leukocyte count: Findings from the Third National Health and Nutrition Examination Survey. Atherosclerosis, 2003. 168(2): p. 351-358.

30. Stocker, R. and J.F. Keaney, Jr., Role of oxidative modifications in atherosclerosis. Physiol Rev, 2004. 84(4): p. 1381-478.

31. Chantler, P.D. and J.C. Frisbee, Arterial function in cardio-metabolic diseases: from the microcirculation to the large conduits. Prog Cardiovasc Dis, 2015. 57(5): p. 489-96.

32. Martinez-Lemus, L.A., et al., Inward remodeling of resistance arteries requires reactive oxygen species-dependent activation of matrix metalloproteinases. Am J Physiol Heart Circ Physiol, 2011. 300(6): p. H2005-15.

33. Raffaitin, C., et al., Metabolic syndrome and risk for incident Alzheimer's disease or vascular dementia: the Three-City Study. Diabetes Care, 2009. 32(1): p. 169-74.

34. Roberts, R.O., et al., Metabolic syndrome, inflammation, and nonamnestic mild cognitive impairment in older persons: a population-based study. Alzheimer Dis Assoc Disord, 2010. 24(1): p. 11-8.

35. Solfrizzi, V., et al., Metabolic syndrome and the risk of vascular dementia: the Italian Longitudinal Study on Ageing. J Neurol Neurosurg Psychiatry, 2010. 81(4): p. 433-40.

36. Chantler, P., Shrader, CD., Tabone, LE., d'Audiffret, AC., Huseynova, K., Brooks, SD., Branyan, KW., Grogg, KA., and Frisbee, JC, Cerebral cortical microvascular rarefaction in metabolic syndrome is dependent on insulin resistance and loss of nitric oxide bioavailability. Microcirulation, 2015. In Press. 
37. Tucsek, Z., et al., Aging exacerbates obesity-induced cerebromicrovascular rarefaction, neurovascular uncoupling, and cognitive decline in mice. J Gerontol A Biol Sci Med Sci, 2014. 69(11): p. 1339-52.

38. Winocur, G., et al., Memory impairment in obese Zucker rats: an investigation of cognitive function in an animal model of insulin resistance and obesity. Behav Neurosci, 2005. 119(5): p. 1389-95.

39. Greenwood, C.E. and G. Winocur, Cognitive impairment in rats fed high-fat diets: a specific effect of saturated fatty-acid intake. Behav Neurosci, 1996. 110(3): p. 451-9.

40. Kanoski, S.E., et al., The effects of energy-rich diets on discrimination reversal learning and on BDNF in the hippocampus and prefrontal cortex of the rat. Behav Brain Res, 2007. 182(1): p. 57-66.

41. Jurdak, N., A.H. Lichtenstein, and R.B. Kanarek, Diet-induced obesity and spatial cognition in young male rats. Nutr Neurosci, 2008. 11(2): p. 48-54.

42. Zhang, L., et al., A rat model of mild cognitive impairment associated with vascular factor. Neuropathology, 2011. 31(2): p. 112-21.

43. Fukui, K., et al., Cognitive impairment of rats caused by oxidative stress and aging, and its prevention by vitamin E. Ann N Y Acad Sci, 2002. 959: p. 275-84.

44. Gage, F.H., P.A. Kelly, and A. Bjorklund, Regional changes in brain glucose metabolism reflect cognitive impairments in aged rats. J Neurosci, 1984. 4(11): p. 2856-65.

45. Eskelinen, M.H., et al., Fat intake at midlife and cognitive impairment later in life: a population-based CAIDE study. Int J Geriatr Psychiatry, 2008. 23(7): p. 741-7.

46. Morris, M.C., et al., Dietary fat intake and 6-year cognitive change in an older biracial community population. Neurology, 2004. 62(9): p. 1573-9.

47. Lu, D., et al., Atorvastatin reduction of intravascular thrombosis, increase in cerebral microvascular patency and integrity, and enhancement of spatial learning in rats subjected to traumatic brain injury. J Neurosurg, 2004. 101(5): p. 813-21.

48. Flint, A.C., et al., Statin use during ischemic stroke hospitalization is strongly associated with improved poststroke survival. Stroke, 2012. 43(1): p. 147-54. 
Table 1. Baseline characteristics of animal groups at 7-8, 12-13 and 16-17 weeks of age. Abbreviations: CAP (captopril), HDZ (hydralazine), MET (metformin), RGZ (rosiglitazone), ATOR (atorvastatin), GEM (gemfibrozil), TEM (TEMPOL), TLM (L-NAME); MAP (mean arterial pressure); TNF- $\alpha$ (tumor necrosis factor); N-tyr (Nitrotyrosine) * $\mathrm{p}<0.05$ vs. LZR; $\uparrow \mathrm{p}<0.05$ vs. OZR.

\begin{tabular}{|c|c|c|c|c|c|c|c|c|c|c|}
\hline & LZR & OZR & $\begin{array}{l}\text { OZR- } \\
\text { CAP }\end{array}$ & $\begin{array}{l}\text { OZR- } \\
\text { HDZ }\end{array}$ & $\begin{array}{l}\text { OZR- } \\
\text { MET }\end{array}$ & OZR-RGZ & $\begin{array}{l}\text { OZR- } \\
\text { ATOR }\end{array}$ & $\begin{array}{l}\text { OZR- } \\
\text { GEM }\end{array}$ & $\begin{array}{l}\text { OZR- } \\
\text { TEM }\end{array}$ & $\begin{array}{l}\text { OZR- } \\
\text { LNM }\end{array}$ \\
\hline Mass (g) & $145 \pm 6$ & $339 \pm 11 *$ & $335 \pm 7 *$ & $344 \pm 9 *$ & $338 \pm 11^{*}$ & $342 \pm 9 *$ & $331 \pm 11^{*}$ & $345 \pm 9 *$ & $350 \pm 10^{*}$ & $338 \pm 11^{*}$ \\
\hline $12-13 W$ & $224 \pm 6$ & $452 \pm 10 *$ & $454 \pm 9 *$ & $450 \pm 10 *$ & $448 \pm 8 *$ & $456 \pm 9 *$ & $453 \pm 11 *$ & $454 \pm 9 *$ & $450 \pm 10^{*}$ & $458 \pm 9 *$ \\
\hline 16-17W & $365 \pm 9$ & $691 \pm 13 *$ & $685 \pm 10^{*}$ & $683 \pm 11 *$ & $692 \pm 13 *$ & $685 \pm 9 *$ & $681 \pm 12 *$ & $685 \pm 12 *$ & $690 \pm 11 *$ & $678 \pm 12 *$ \\
\hline MAP (mmHg) 7-8W & $104 \pm 4$ & $103 \pm 7$ & $102 \pm 6$ & $99 \pm 5$ & $101 \pm 8$ & $103 \pm 5$ & $101 \pm 4$ & $105 \pm 6$ & $104 \pm 6$ & $106 \pm 4$ \\
\hline 12-13W & $105 \pm 5$ & $114 \pm 5$ & $98 \pm 5 \dagger$ & $97 \pm 5 \dagger$ & $113 \pm 4$ & $112 \pm 4$ & $106 \pm 5$ & $109 \pm 6$ & $100 \pm 4 \dagger$ & $131 \pm 6 * \dagger$ \\
\hline 16-17W & $103 \pm 6$ & $135 \pm 5^{*}$ & $107 \pm 6 \dagger$ & $101 \pm 6 \dagger$ & $129 \pm 6^{*}$ & $130 \pm 6^{*}$ & $114 \pm 6 \dagger$ & $119 \pm 5^{* \dagger}$ & $109 \pm 6 \dagger$ & $142 \pm 6^{*}$ \\
\hline Gluc (mg/dl) $7-8 W$ & $84 \pm 5$ & $99 \pm 6$ & $94 \pm 4$ & $94 \pm 5$ & $88 \pm 7$ & $84 \pm 6$ & $90 \pm 5$ & $88 \pm 5$ & $90 \pm 5$ & $94 \pm 6$ \\
\hline 12-13W & $92 \pm 5$ & $121 \pm 5 *$ & $116 \pm 6^{*}$ & $120 \pm 7 *$ & $101 \pm 4 \dagger$ & $94 \pm 6 \dagger$ & $119 \pm 5^{*}$ & $121 \pm 6^{*}$ & $112 \pm 5^{*}$ & $123 \pm 5^{*}$ \\
\hline 16-17W & $94 \pm 4$ & $148 \pm 8 *$ & $141 \pm 9 *$ & $147 \pm 7 *$ & $116 \pm 5^{*} \dagger$ & $114 \pm 5 * \dagger$ & $145 \pm 6^{*}$ & $139 \pm 6^{*}$ & $134 \pm 6^{*}$ & $151 \pm 6^{*}$ \\
\hline Insulin (ng/ml) 7-8W & $0.9 \pm 0.3$ & $5.4 \pm 0.5^{*}$ & $5.2 \pm 0.4^{*}$ & $5.5 \pm 0.5^{*}$ & $5.3 \pm 0.6^{*}$ & $5.1 \pm 0.7 *$ & $5.5 \pm 0.6^{*}$ & $5.4 \pm 0.5^{*}$ & $5.5 \pm 0.6^{*}$ & $5.4 \pm 0.4^{*}$ \\
\hline 12-13W & $1.2 \pm 0.2$ & $6.9 \pm 0.5^{*}$ & $5.5 \pm 0.5^{*}$ & $6.8 \pm 0.6^{*}$ & $5.0 \pm 0.6^{* \dagger}$ & $4.8 \pm 0.5^{* \dagger}$ & $6.3 \pm 0.5^{*}$ & $6.5 \pm 0.6^{*}$ & $5.2 \pm 0.6^{* \dagger}$ & $7.1 \pm 0.4^{*}$ \\
\hline 16-17W & $1.4 \pm 0.4$ & $8.8 \pm 0.6^{*}$ & $6.8 \pm 0.5^{* \dagger}$ & $8.4 \pm 0.6^{*}$ & $5.5 \pm 0.6^{* \dagger}$ & $5.2 \pm 0.4 * \dagger$ & $7.3 \pm 0.6^{* \dagger}$ & $7.7 \pm 0.6^{*}$ & $6.5 \pm 0.5^{* \dagger}$ & $9.4 \pm 0.5^{*}$ \\
\hline Chol (mg/dl) $\quad 7-8 W$ & $76 \pm 6$ & $84 \pm 8$ & $88 \pm 7$ & $94 \pm 11$ & $83 \pm 8$ & $90 \pm 9$ & $79 \pm 5$ & $82 \pm 7$ & $79 \pm 8$ & $84 \pm 8$ \\
\hline 12-13W & $81 \pm 5$ & $104 \pm 6 *$ & $106 \pm 5 *$ & $105 \pm 7 *$ & $110 \pm 9 *$ & $109 \pm 9 *$ & $89 \pm 5 \dagger$ & $86 \pm 5 \dagger$ & $98 \pm 6^{*}$ & $106 \pm 6^{*}$ \\
\hline 16-17W & $86 \pm 6$ & $134 \pm 9 *$ & $130 \pm 6^{*}$ & $134 \pm 11 *$ & $133 \pm 10 *$ & $129 \pm 11 *$ & $96 \pm 5 \dagger$ & $102 \pm 7 \dagger$ & $129 \pm 9 *$ & $138 \pm 11^{*}$ \\
\hline N-tyr (ng/ml) 7-8W & $8 \pm 3$ & $15 \pm 4$ & $13 \pm 4$ & $16 \pm 5$ & $15 \pm 4$ & $17 \pm 5$ & $14 \pm 4$ & $15 \pm 5$ & $13 \pm 4$ & $16 \pm 5$ \\
\hline 12-13W & $11 \pm 3$ & $34 \pm 5^{*}$ & $29 \pm 4 *$ & $33 \pm 5^{*}$ & $24 \pm 4 *$ & $22 \pm 5 * \dagger$ & $15 \pm 4 \dagger$ & $23 \pm 4 * \dagger$ & $13 \pm 4 \dagger$ & $36 \pm 5^{*}$ \\
\hline 16-17W & $15 \pm 4$ & $56 \pm 6^{*}$ & $42 \pm 5^{* \dagger}$ & $49 \pm 7 *$ & $46 \pm 7 *$ & $39 \pm 5 * \dagger$ & $38 \pm 4 * \dagger$ & $46 \pm 7 *$ & $27 \pm 4 * \dagger$ & $66 \pm 8 *$ \\
\hline
\end{tabular}




\begin{tabular}{|c|c|c|c|c|c|c|c|c|c|c|}
\hline TNF- $\alpha(\mathrm{pg} / \mathrm{ml}) 7-8 \mathrm{~W}$ & $1.1 \pm 0.4$ & $3.4 \pm 0.5^{*}$ & $3.2 \pm 0.6^{*}$ & $3.5 \pm 0.5^{*}$ & $2.9 \pm 0.5^{*}$ & $3.1 \pm .4 *$ & $3.2 \pm 0.4^{*}$ & $3.5 \pm 0.5^{*}$ & $2.8 \pm 0.4^{*}$ & $3.3 \pm 0.6^{*}$ \\
\hline $12-13 W$ & $1.8 \pm 0.2$ & $10.4 \pm 1.0^{*}$ & $9.4 \pm 0.8^{*}$ & $9.5 \pm 0.6^{*}$ & $7.2 \pm 0.5^{* \dagger}$ & $8.0 \pm 0.4 * \dagger$ & $6.2 \pm 0.5 * \dagger$ & $7.5 \pm 0.5^{*} \dagger$ & $5.8 \pm 0.5 * \dagger$ & $10.8 \pm 0.6^{*}$ \\
\hline $16-17 \mathrm{~W}$ & $2.6 \pm 0.4$ & $13.1 \pm 1.0^{*}$ & $10.2 \pm 0.6^{*} \dagger$ & $12.5 \pm 0.7 *$ & $9.2 \pm 0.6 * \dagger$ & $8.6 \pm 0.7 * \dagger$ & $7.2 \pm 0.6 * \dagger$ & $9.5 \pm 0.5 * \dagger$ & $5.0 \pm 0.6 * \dagger$ & $14.3 \pm 1.1^{*}$ \\
\hline MCP-1 (pg/ml) 7-8W & $29 \pm 4$ & $35 \pm 6$ & $34 \pm 5$ & $37 \pm 4$ & $30 \pm 5$ & $31 \pm 6$ & $34 \pm 6$ & $33 \pm 5$ & $30 \pm 4$ & $36 \pm 5$ \\
\hline $12-13 W$ & $30 \pm 5$ & $90 \pm 10^{*}$ & $68 \pm 6 * \dagger$ & $87 \pm 5^{*}$ & $74 \pm 6 * \dagger$ & $71 \pm 5 * \dagger$ & $64 \pm 6 * \dagger$ & $73 \pm 5 * \dagger$ & $54 \pm 6 * \dagger$ & $96 \pm 6 * \dagger$ \\
\hline $16-17 \mathrm{~W}$ & $38 \pm 6$ & $104 \pm 8 *$ & $80 \pm 5^{* \dagger}$ & $90 \pm 9 *$ & $84 \pm 8^{*}$ & $71 \pm 7 * \dagger$ & $64 \pm 6 * \dagger$ & $93 \pm 9 *$ & $58 \pm 6 * \dagger$ & $116 \pm 10^{*}$ \\
\hline
\end{tabular}




\section{FIGURE LEGENDS}

Figure 1. Ex vivo middle cerebral artery wall mechanics and reactivity in LZR and OZR at 7-8 weeks, 1213 weeks and 16-17 weeks of age. Data are presented for MCA inner diameter with passive expansion (Panel A) and circumferential stress versus strain relationships (Panel B), dilator responses to increasing concentrations of acetylcholine (Panel C), myogenic activation (Panel D) and vascular nitric oxide bioavailability with increasing concentrations of methacholine (Panel E). All data are presented as mean \pm SE. For Panels $\mathrm{A}-\mathrm{D},{ }^{*} \mathrm{p}<0.05$ versus responses in LZR at 7-8 weeks of age; $\uparrow \mathrm{p}<0.05$ versus responses in LZR at 12-13 weeks of age. $\$ p<0.05$ versus responses in OZR at 7-8 weeks of age; $\# \mathrm{p}<0.05$ versus responses in OZR at 12-13 weeks of age.Please see text for additional details and formulae. For Panel E, * $\mathrm{p}<0.05$ versus responses in LZR at that age; $\uparrow \mathrm{p}<0.05$ versus responses in OZR at that age.

Figure 2. Ex vivo middle cerebral artery wall mechanics and reactivity in LZR and OZR at 7-8 weeks, 1213 weeks and 16-17 weeks of age. Data are presented for responses following treatment with antihypertensive agents: captopril (CAP) or hydralazine (HDZ) starting at 8 weeks of age. Data are presented for MCA maximum inner diameter with passive expansion (Panel A) and the slope ( $\beta$ ) coefficient for circumferential stress versus strain relationships (Panel B), the upper bound of the acetylcholine concentration-response relation (Panel C), the slope ( $\beta$ ) coefficient for myogenic activation (Panel D) and the slope $(\beta)$ coefficient for vascular nitric oxide bioavailability with increasing concentrations of methacholine (Panel E). All data are presented as mean \pm SE. $* \mathrm{p}<0.05$ versus responses in LZR at that age; $\uparrow \mathrm{p}<0.05$ versus responses in OZR at that age; $\$ \mathrm{p}<0.05$ versus responses under that condition at 12 13 weeks of age. Please see text for additional details and formulae.

Figure 3. Ex vivo middle cerebral artery wall mechanics and reactivity in LZR and OZR at 7-8 weeks, 1213 weeks and 16-17 weeks of age. Data are presented for responses following treatment with anti-diabetes agents: rosiglitazone (ROSI) or metformin (MET) starting at 8 weeks of age. Data are presented for MCA 
maximum inner diameter with passive expansion (Panel A) and the slope $(\beta)$ coefficient for circumferential stress versus strain relationships (Panel B), the upper bound of the acetylcholine concentration-response relation (Panel C), the slope $(\beta)$ coefficient for myogenic activation (Panel D) and the slope $(\beta)$ coefficient for vascular nitric oxide bioavailability with increasing concentrations of methacholine (Panel E). All data are presented as mean \pm SE. $* \mathrm{p}<0.05$ versus responses in LZR at that age; $\uparrow \mathrm{p}<0.05$ versus responses in OZR at that age; $\$ p<0.05$ versus responses under that condition at 12-13 weeks of age. Please see text for additional details and formulae.

Figure 4. Ex vivo middle cerebral artery wall mechanics and reactivity in LZR and OZR at 7-8 weeks, 1213 weeks and 16-17 weeks of age. Data are presented for responses following treatment with antidyslipidemia agents: atorvastatin (ATOR) or gemfibrozil (GEM) starting at 8 weeks of age. Data are presented for MCA maximum inner diameter with passive expansion (Panel A) and the slope ( $\beta$ ) coefficient for circumferential stress versus strain relationships (Panel B), the upper bound of the acetylcholine concentration-response relation (Panel C), the slope $(\beta)$ coefficient for myogenic activation (Panel D) and the slope $(\beta)$ coefficient for vascular nitric oxide bioavailability with increasing concentrations of methacholine (Panel E). All data are presented as mean \pm SE. $* \mathrm{p}<0.05$ versus responses in LZR at that age; $\dagger \mathrm{p}<0.05$ versus responses in OZR at that age; $\$ \mathrm{p}<0.05$ versus responses under that condition at $12-13$ weeks of age. Please see text for additional details and formulae.

Figure 5. Ex vivo middle cerebral artery wall mechanics and reactivity in LZR and OZR at 7-8 weeks, 1213 weeks and 16-17 weeks of age. Data are presented for responses following treatment with the antioxidant TEMPOL (TEM) or the nitric oxide synthase inhibitor (NOS) starting at 8 weeks of age. Data are presented for MCA maximum inner diameter with passive expansion (Panel A) and the slope $(\beta)$ coefficient for circumferential stress versus strain relationships (Panel B), the upper bound of the acetylcholine 
concentration-response relation (Panel C), the slope $(\beta)$ coefficient for myogenic activation (Panel D) and the slope $(\beta)$ coefficient for vascular nitric oxide bioavailability with increasing concentrations of methacholine (Panel E). All data are presented as mean \pm SE. $* \mathrm{p}<0.05$ versus responses in LZR at that age; $\dagger \mathrm{p}<0.05$ versus responses in OZR at that age; $\ddagger \mathrm{p}<0.05$ versus responses under that condition at 12-13 weeks of age. Please see text for additional details and formulae.

Figure 6. Relationships between vascular nitric oxide bioavailability, dilator reactivity to acetylcholine and wall mechanics in LZR and OZR at 7-8 weeks (Panel A), 12-13 weeks (Panel B) and 16-17 weeks (Panel C) of age. Data are presented for the results of individual experiments for LZR under control conditions and OZR under control conditions and following treatment with captopril (CAP), hydralazine (HDZ), rosiglitazone (ROSI), metformin (MET), atorvastatin (ATOR), gemfibrozil (GEM) and TEMPOL (TEM). Vascular nitric oxide bioavailability is presented as the slope $(\beta)$ coefficient for vascular nitric oxide concentration with increasing concentrations of methacholine, dilator reactivity is presented as the upper bound of the acetylcholine concentration-response relationship for ex vivo MCA, and wall mechanics are presented as the slope $(\beta)$ coefficient for the stress versus strain relationship. Please see text for additional details and formulae.

Figure 7. Relationships between the slope $(\beta)$ coefficient for circumferential stress versus strain relationships and the mean arterial pressure for LZR and OZR at 16-17 weeks of age. Data are presented as the aggregate for the results from LZR under control conditions and OZR under control conditions and following treatment with captopril (CAP), hydralazine (HDZ), rosiglitazone (ROSI), metformin (MET), atorvastatin (ATOR), gemfibrozil (GEM), TEMPOL (TEM) and L-NAME (LNM). All data are presented as mean $\pm \mathrm{SE}$, and the linear regression equation of best fit is represented as: $y=-0.259+0.021 x ; r^{2}=0.724$. 

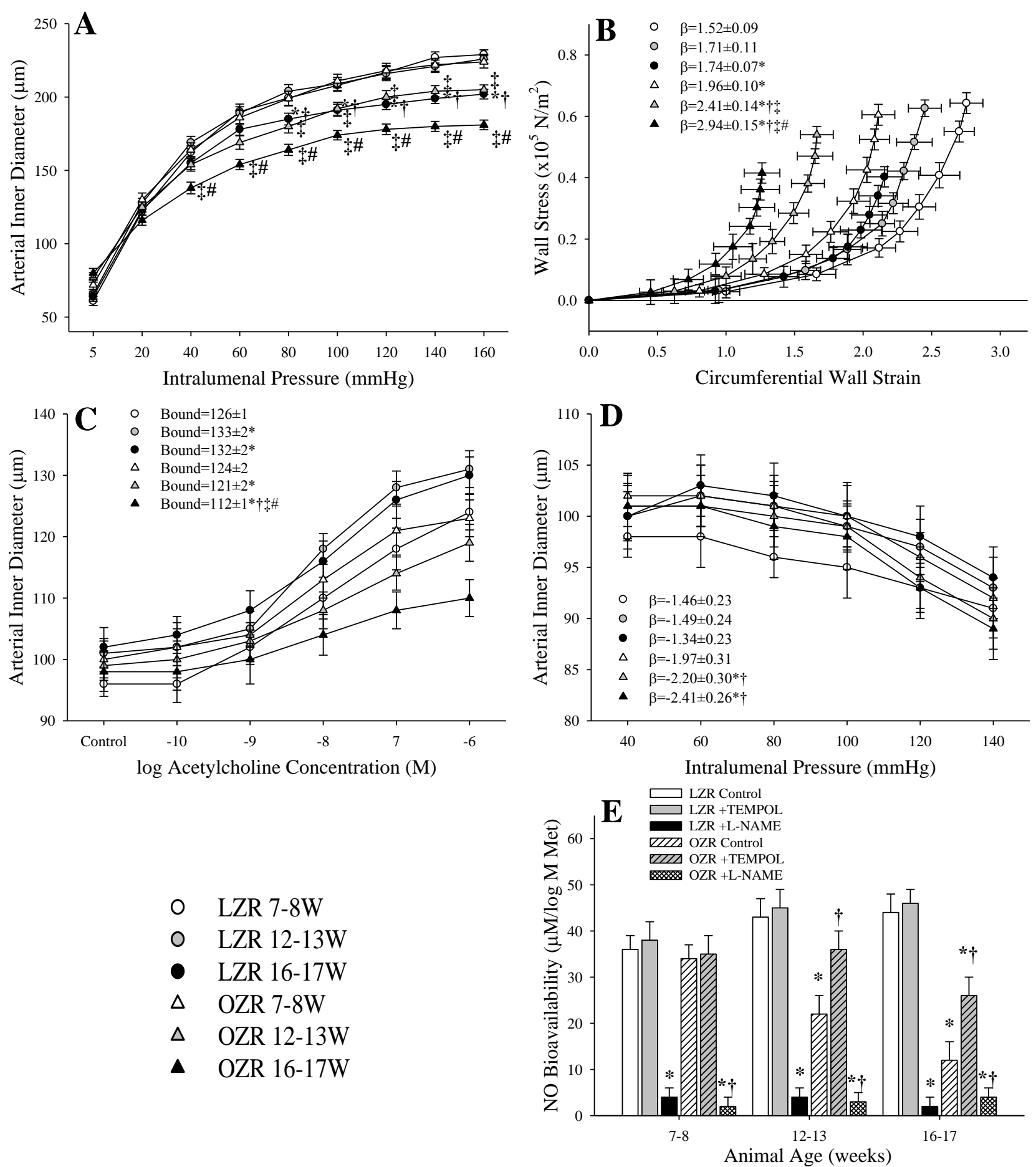

Figure 1. (Control Data)

Brooks et al. 

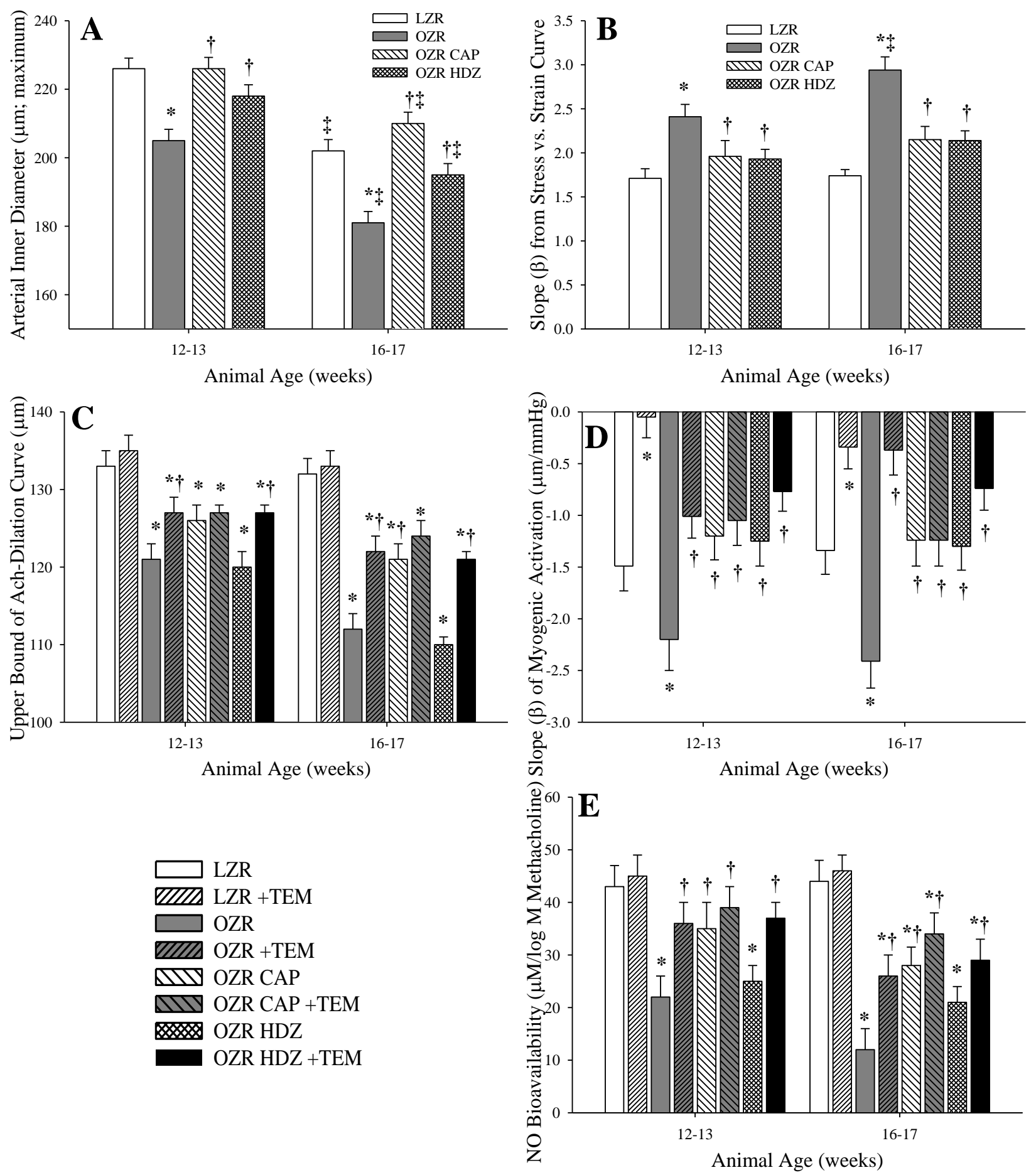

Figure 2. (Anti-hypertensive) Brooks et al. 

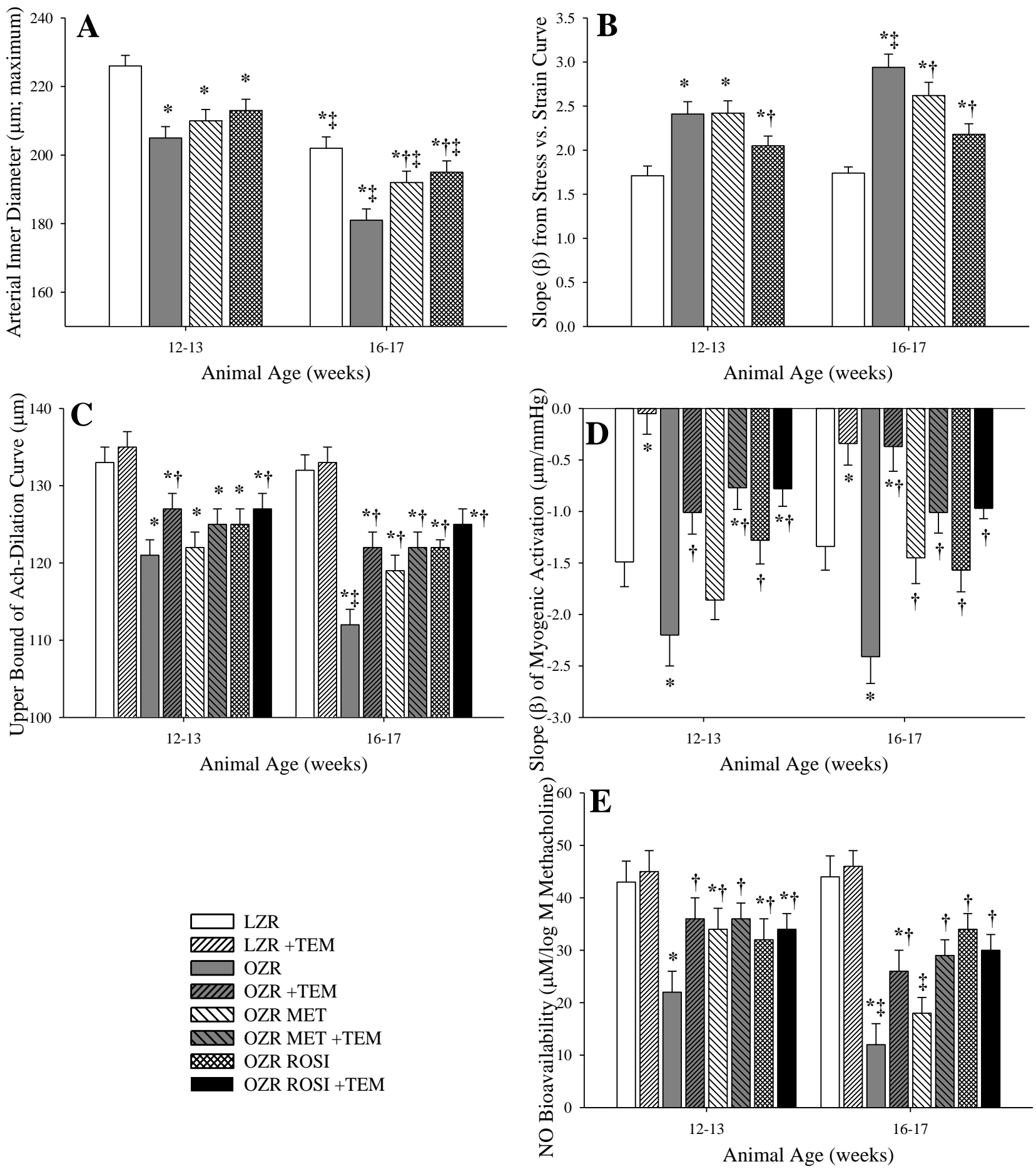

Figure 3. (Anti-Diabetic)

Brooks et al. 

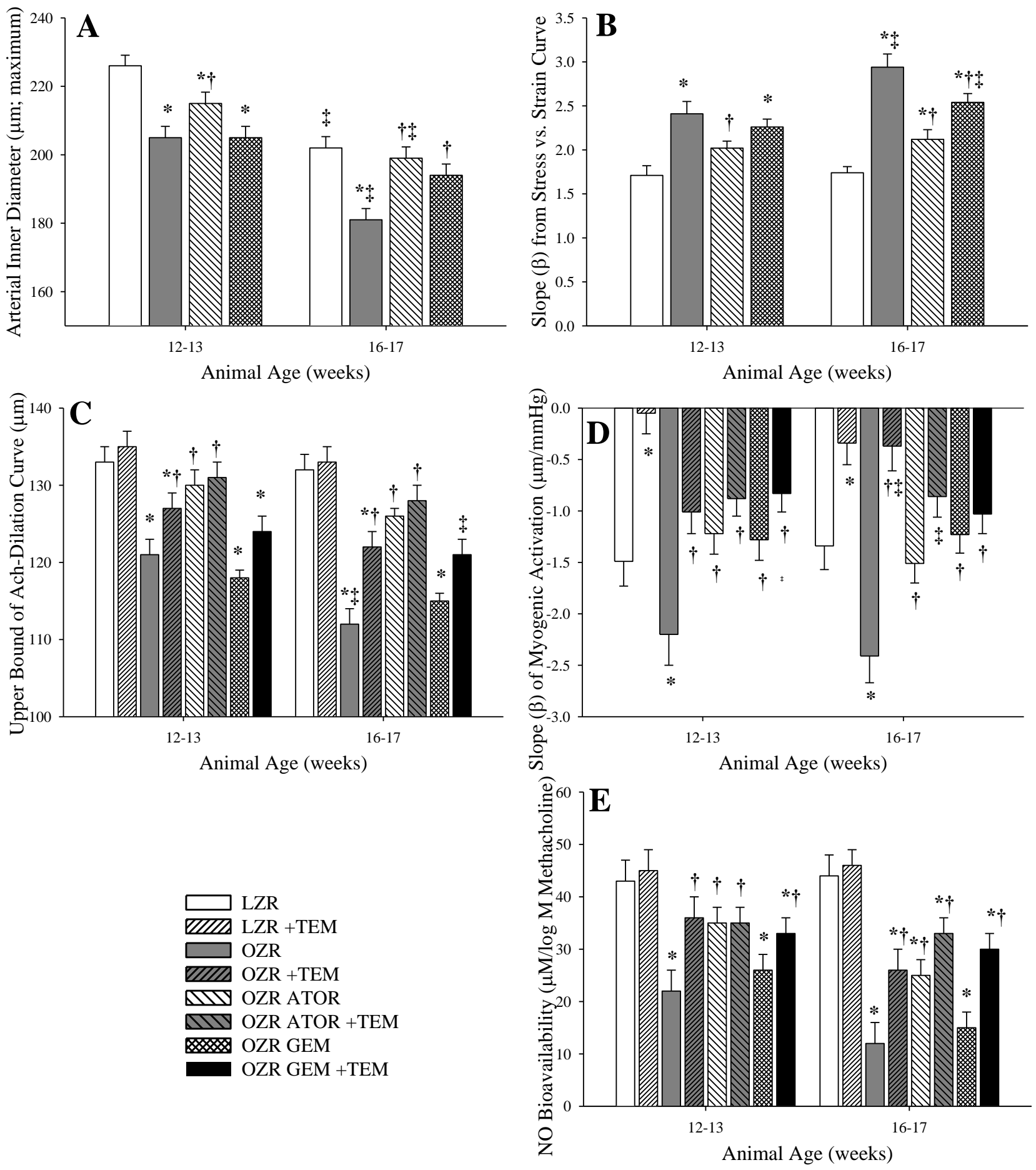

Figure 4. (Anti-Dyslipidemia)

Brooks et al. 

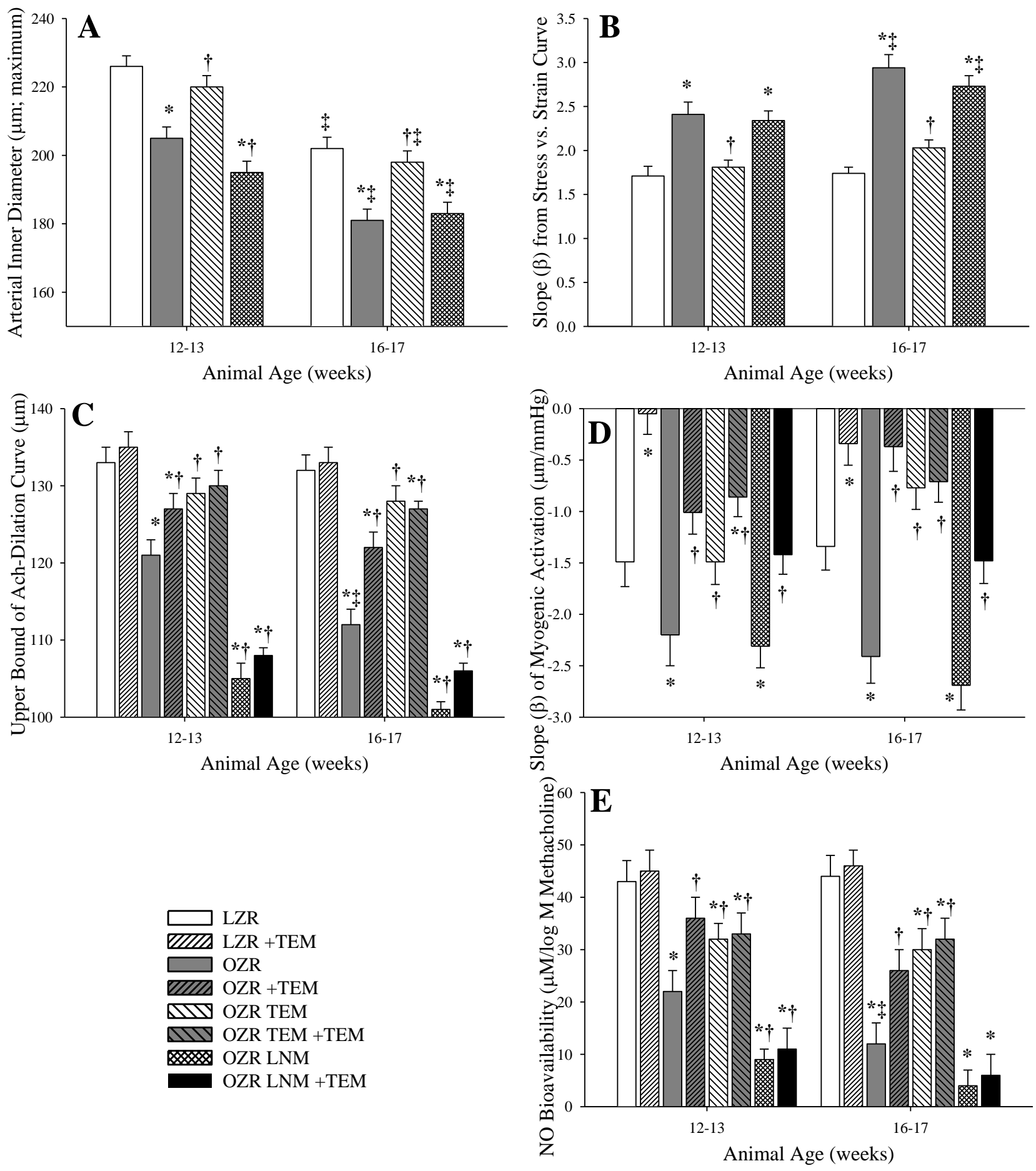

Figure 5. (Anti-Oxidant)

Brooks et al. 

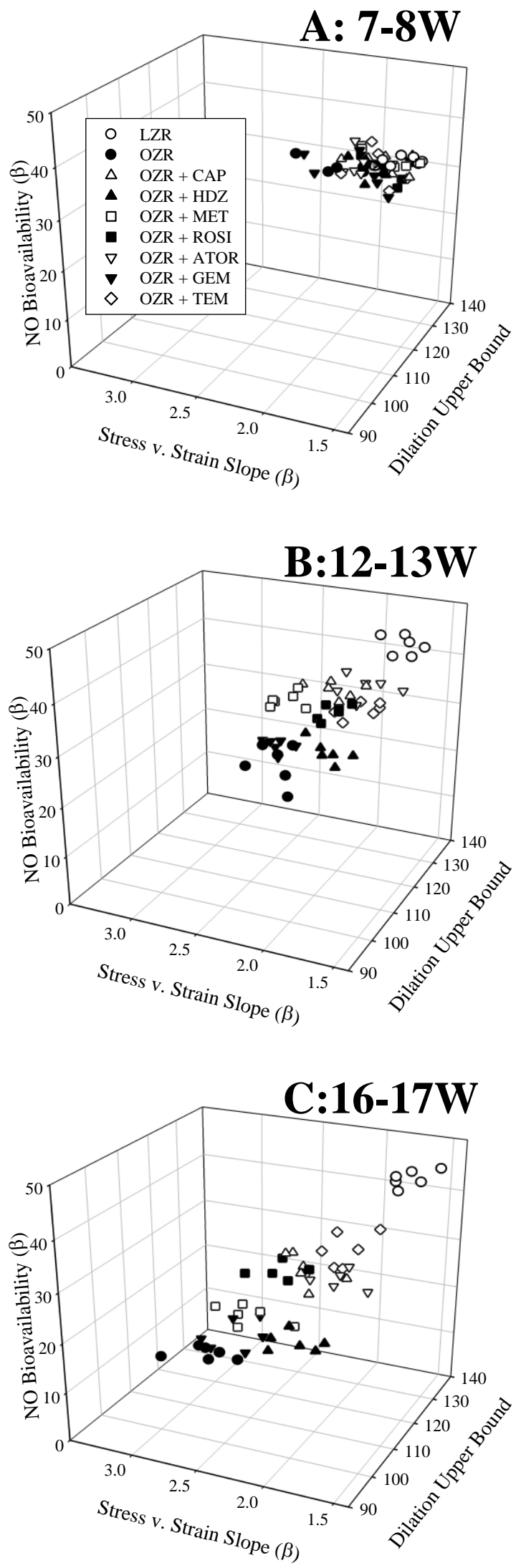

Figure 6. (3D Correlations)

Brooks et al. 


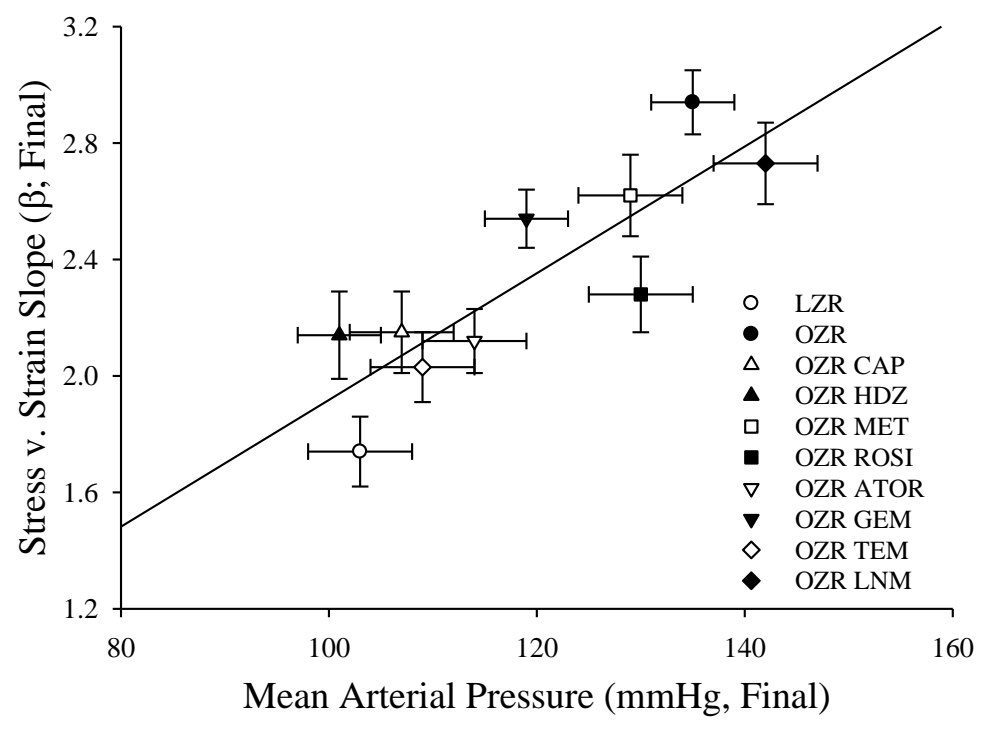

Figure 7. (SS $\beta$ vs. MAP)

Brooks et al. 


\section{Chapter 3: Conceptual Framework}

The most significant finding of this chapter was that interventions which increased NO bioavailability also significantly improved vascular reactivity, reduced inflammatory biomarkers (TNF$\alpha$ and MCP-1), and reduced nitrotyrosine, a plasma biomarker for oxidative stress. Rosiglitazone, atorvastatin, captopril, and TEMPOL were the most effective interventions at achieving these outcomes, with TEMPOL and atorvastatin having the largest benefit. Chronic treatment with L-NAME, conversely, resulted in the most severe manifestation of each of the vascular pathologies. Temporal analysis of the interaction between NO bioavailability, vascular reactivity, and vascular stiffness reveal a progressive and concurrent decrease in each of these metrics from 7 to 17 weeks in OZR; animals from each treatment group fit neatly into the three-dimensional model, with the most efficacious treatments at preserving NO bioavailability trending towards values for LZR, while the least efficacious treatments trended towards values for untreated OZR. As was found in chapter 1, the increase in oxidative stress and inflammation that occurs with the onset of MetSyn in OZR significantly impaired functional and structural components of the cerebral circulation.

he second and third chapters of this dissertation provide a substantial body of evidence that development of MetSyn leads to structural and functional impairments in the middle cerebral arteries, as well as a progressive loss of penetrating and parenchymal arterioles and capillaries within the cortex. The next step was to evaluate how these cerebrovascular impairments, inherent in OZR, would impact the pathophysiology of prevalent cerebrovascular diseases. The next chapter of this dissertation investigates the vascular and neurological outcomes to ischemic stroke in LZR and OZR, and attempts to elucidate distinct mechanisms contributing to both the acute stroke injury as well as the long-term vascular complications from ischemic stroke. 


\section{CHAPTER 4:}

OXIDATIVE STRESS EXACERBAES VASCULAR DYSFUNCTION AND INFARCT SIZE AFTER ISCHEMIC STROKE IN A RODENT MODEL OF METABOLIC SYNDROME

Steven Brooks ${ }^{1,5}$, Shinichi Asano ${ }^{2,5}$, Evan DeVallance ${ }^{2,5}$, Kayla Branyan ${ }^{2,5}$, Heng Hu ${ }^{4}$, Xuefang

$$
\text { Ren }^{4} \text {, Jefferson C. Frisbee }{ }^{1,3,5} \text { and Paul D. Chantler }{ }^{2,3,5}
$$

Department of Physiology and Pharmacology ${ }^{1}$, Division of Exercise Physiology ${ }^{2}$, Clinical and Translational Science Institute ${ }^{3}$, Experimental Stroke Core Facility4, Center for Cardiovascular and Respiratory Sciences ${ }^{5}$; West Virginia University Health Sciences Center, Morgantown, WV

Send Correspondence to:

Jefferson C. Frisbee, Ph.D.

Department of Physiology and Pharmacology

Center for Cardiovascular and Respiratory Sciences

Robert C. Byrd Health Sciences Center

PO Box 9227

West Virginia University School of Medicine

Morgantown, WV 26505

Phone: (304) 293-6527

Fax: 304-293-5513

Email: jefrisbee@hsc.wvu.edu

*This chapter presents data and analysis from a pilot study

Authorship Statement: SD Brooks contributed to this manuscript in terms of original idea, data collection, data analysis, primary synthesis of manuscript, and manuscript review. 


\section{ABSTRACT}

Objective: Metabolic syndrome (MetSyn) may exacerbate ischemic stroke-induced vascular dysfunction and reduce vascularization, leading to larger infarcts and poor long term recovery. This study sought to evaluate vascular and neurological impairments from transient middle cerebral artery occlusion (tMCAO) in obese Zucker rats (OZR), a model of MetSyn, and investigate acute inhibition of $\mathrm{NAD}(\mathrm{P}) \mathrm{H}$ oxidase 2 (NOX2) with gp9ds-tat as a candidate for reducing oxidative injury during stroke.

Methods: 17 weeks old OZR were sacrificed at 24 hours or 15 days following tMCAO. A separate cohort received infusion of gp91ds-tat $(10 \mathrm{mg} / \mathrm{kg}$ body weight) during tMCAO and was sacrificed at 15 days. Reactivity of ipsilateral (IL) and contralateral (CL) middle cerebral arteries (MCA) was assessed by ex vivo pressure myography. Nitric oxide (NO) production was measured by DAF assay and infarct size by TTC straining. Cerebral microvessel density was measured by staining with anti-CD31 antibody

Results: Dilation to acetylcholine of lean Zucker (LZR) MCAs was reduced 50\% compared to sham LZR at 24 hours, and another 20\% in IL MCA at 15 days. In OZR, IL-MCA reactivity was reduced $50 \%$ compared to OZR sham at 24 hours. By 15 days, OZR IL-MCA reactivity was completely ablated, while CL-MCA reactivity had decreased 30\%. NO bioavailability in LZR and OZR at 24 hours was reduced 35\%, and fell 50\% further in OZR at 15 days. Rates of mortality and infarct size were higher for OZR at both time points. Treatment of OZR with gp91ds-tat completely blocked the progressive loss of IL/CL MCA reactivity from 24 hrs-15 days, improved post-stroke cerebral blood flow, and reduced mortality. At 15 days after stroke, both LZR and OZR showed significant rarefaction of microvessels in the ischemic core, but only OZR showed rarefaction of cerebral microvessels in the penumbra. 
Conclusions: MetSyn increases infarct size and mortality, and progressively exacerbated strokeinduced vascular dysfunction in a preclinical model of ischemic stroke. The worsened vascular and neurological outcomes reflect oxidative injury during ischemia/reperfusion, and were successfully prevented via treatment with gp91ds-tat during tMCAO. Inhibition of Nox2 during stroke to control oxidative damage may therefore present a novel therapeutic opportunity for improving ischemic stroke outcomes. 


\section{INTRODUCTION}

Ischemic Stroke is among the leading causes of death and disease burden worldwide. According to the AHA's 2016 Heart Disease and Stroke Statistics update, around 800,000 people will experience a stroke every year in the US; a stroke occurs on average every 40 seconds, and approximately 15 people die every hour from stroke [1-4](AHA). Stroke is one of the leading causes of serious long-term disability and decreased quality of life, and alone is responsible for a disease cost burden of over $\$ 34$ billion in the US annually $[4,5](\mathrm{CDC})$. Despite its prevalence, treatment options for patients are very limited. Currently, there are only two FDA-approved treatments for ischemic stroke[3]. The "gold standard" treatment is intravenous recombinant tissue plasminogen activator (IV tPA), first approved for use in 1996, which works to dissolve the blood clot and restore blood flow to the ischemic area [6, 7]. However, IV tPA usage is restricted by a narrow treatment window ( 0 to 3-4.5 hours after symptom onset), as well as contra-indicated for use in many patients due to elevated hemorrhage risk. As a result, IV tPA is vastly under-utilized, with estimates of usage ranging from only $3 \%-7.0 \%[3,8]$ of stroke patients.

The US Centers for Disease Control names high blood pressure, high cholesterol, heart disease, and diabetes as significant independent risk factors for stroke, and over $40 \%$ of stroke patients are hyperglycemic at time of event $[5,9-14]$. The metabolic syndrome (MetSyn), which encompasses each of these risk factors as constituents, is itself a significant risk factor [10, 1517]. MetSyn is defined clinically as the comorbid presence of three or more risk factors, including: central obesity, hypertriglyceridemia, high cholesterol, hypertension, and hyperglycemia $[15,16]$. Data from the NHANES study reveals that over $35 \%$ of all adults, and over $50 \%$ adults aged 60 or older, are estimated to have the metabolic syndrome [16]. 
The obese Zucker rat (OZR; $f a / f a)$ is a translationally relevant rodent model for MetSyn, with demonstrated history for studying cerebrovascular consequences of MetSyn and its underlying network of systemic pathologies [18-20]. We have previously reported that middle cerebral arteries (MCA) from OZR develop significant alterations in myogenic tone [21], vascular reactivity [22], and vessel wall mechanics [22, 23]. We also recently reported a progressive rarefaction of cerebral cortical microvessels in OZR that was correlated with insulin resistance and nitric oxide (NO) bioavailability [23]. These combined pathologies should theoretically seriously impair blood flow during and after ischemic stroke[24-26]; indeed the only previous studies on stroke in OZR found that ischemic stroke results in increased infarct size, elevation of MCA myogenic tone, and inward vascular remodeling of MCAs at 24 hours following ischemic stroke [27] through hypertension-dependent mechanisms [28]. Additionally, stroke in diabetic mice [24] and rats [29-33] have shown increases in infarct size, edema, and hemorrhage.

The mechanisms through which cerebrovascular dysfunction develops in MetSyn are largely mediated by oxidative stress and inflammation [22, 25, 33-39]. Reactive oxygen species (ROS), which are associated with hypertension, diabetes, and dyslipidemia, react with and reduce the bioavailability of $\mathrm{NO}$, impairing reactivity of pial and parenchymal arterials[40-43]. Inflammation can exacerbate ROS generation, increase neurovascular permeability, and increase neurotoxicity following stroke [44-50],). During stroke, ROS have been shown to cause neuronal death and increase the size of the ischemic core [43, 51-53]. The main sources of ROS in the cerebral circulation are $\mathrm{NAD}(\mathrm{P}) \mathrm{H}$ oxidase enzymes [53-56], especially isoforms Nox2, present in the cerebrovascular endothelium $[50,53,57-60]$ and Nox4 [55, 61-64]. Nox2 is also prevalent in leukocytes [65], specifically neutrophils and macrophages [58], which are elevated during and 
immediately following ischemic stroke $[35,45,66,67]$. The presence of Nox 2 in both the cerebrovascular endothelium, and within the primary immune cells responding acutely to ischemic stroke, implies that it may be a critical source of ROS during ischemia-reperfusion, and may therefore be an ideal therapeutic target.

Maintenance of cerebral blood flow during and after ischemic stroke is essential to prevent neuronal death and limit spread of injury, and following ischemia/reperfusion, increased blood flow and angiogenesis are necessary to promote recovery and rehabilitation [25, 68-71]. As most patients admitted for stroke exhibit one or more of the constituent underlying pathologies of MetSyn [10, 15-17, 72], it is critical that we refine our understanding of how the pre-existing cerebrovascular impairments associated with MetSyn influence vascular and neurological outcomes following ischemic stroke. To address this issue, this study sought to characterize vascular dysfunction and neurological impairments in OZR following 60 minute experimental ischemic stroke, and evaluate the therapeutic benefits of reducing acute oxidative injury during ischemia-reperfusion by inhibiting Nox 2 with the peptide gp91ds-tat during stroke.

Please note: The data and analysis presented within this manuscript reflect a pilot study only, and are not to be evaluated as a completed, fully powered study.

\section{MATERIALS AND METHODS}

Animals: Male LZR and OZR (Harlan) arrived at the West Virginia University Health Sciences Center at 10 weeks of age and were allowed to mature to 17 weeks. Animals were fed standard chow and tap water ad libitum for all experiments. All rats were housed in the animal care facility at the WVU HSC, and all protocols received prior IACUC approval. At 17 weeks of age, LZR ( $\mathrm{n}=4$ for each group) and OZR ( $\mathrm{n}=4$ for each group) underwent either 60 minutes of 
transient middle cerebral artery occlusion (tMCAO) or a sham surgical procedure. Animals were sacrificed at 24 hours post-stroke or 15 days post stroke. A separate cohort of OZR $(n=3)$ received an infusion of gp91ds-tat (10mg/kg body weight) at exactly 30 minutes into 60 minute tMCAO, and were sacrificed at 15 days post-stroke.

Experimental tMCAO: Ischemic stroke was performed using the transient middle cerebral artery occlusion protocol. Briefly, animals were sedated by inhalational isoflurane and maintained on a warming pad. A laser Doppler flowmetry probe (LDF; Moor Instrument) was affixed to the skull over the perfusion territory of the right Middle Cerebral Artery (MCA) to monitor blood flow. The bifurcation of the common carotid artery was isolated, and blood flow temporarily stopped by vessel clamps. The external carotid was dissected, and a 3-0 monofilament was inserted distally. The filament was then extended via internal carotid into the Circle of Willis, and advanced until a minimum $70 \%$ reduction of blood flow in the MCA region was measured by LDF. At 30 minutes into tMCAO, select OZR received either gp91ds-tat (10mg/kg body weight) by IV infusion, or vehicle saline infusion. After 60 minutes of occlusion, the monofilament was removed; restoration of blood flow was confirmed by LDF. The external carotid was then ligated and cauterized, and the surgical opening was sutured shut. 4 hours post tMCAO onset, the animal was evaluated behaviorally for stroke severity

Animals were sacrificed at 24 hours of 15 days post-stroke. Prior to sacrifice, animals were anesthetized with injections of sodium pentobarbital $(50 \mathrm{mg} / \mathrm{kg}$ i.p.). While under anesthesia, animals received tracheal intubation to facilitate maintenance of a patent airway. In all rats, a carotid artery and an external jugular vein were cannulated for determination of arterial pressure and for infusion of additional substances, respectively, as necessary (e.g., anesthetic, 
heparin, etc.). While under anesthetic, an aliquot of blood was drawn from the jugular vein to be used for the subsequent determination of plasma biomarkers.

While deeply anesthetized, each rat was decapitated, after which the brain was removed from the skull and placed in cold physiological salt solution (PSS; $4^{\circ} \mathrm{C}$ ). Both the ipsilateral (IL) and contralateral (CL) middle cerebral arteries (MCA) were dissected from their origin at the Circle of Willis and placed into clean dishes of cold PSS. The brains were then immediately sectioned and stained with triphenyltetrazolium chloride (TTC) to assess the presence and size of any infarction. Each MCA was subsequently doubly cannulated within in a heated chamber $\left(37^{\circ} \mathrm{C}\right)$ that allowed the lumen and exterior of the vessel to be perfused and superfused, respectively, with PSS from separate reservoirs. The PSS was equilibrated with a $21 \% \mathrm{O}_{2}, 5 \%$ $\mathrm{CO}_{2}$, and $74 \% \mathrm{~N}_{2}$ gas mixture and had the following composition $(\mathrm{mM}): 119 \mathrm{NaCl}, 4.7 \mathrm{KCl}$, $1.17 \mathrm{MgSO}_{4}, 1.6 \mathrm{CaCl}_{2}, 1.18 \mathrm{NaH}_{2} \mathrm{PO}_{4}, 24 \mathrm{NaHCO}_{3}, 0.026 \mathrm{EDTA}$, and 5.5 glucose. Any side branches were ligated using a single strand teased from 6-0 suture. Vessel diameter was measured using television microscopy and an on-screen video micrometer.

Measurements of Vascular Reactivity in Isolated MCA: Following cannulation, MCAs were extended to their in situ length and were equilibrated at $80 \%$ of the animal's mean arterial pressure to approximate in vivo perfusion pressure (Lombard paper). Any vessel that did not demonstrate significant active tone at the equilibration pressure was discarded. Active tone at the equilibration pressure was calculated as $\left(\Delta \mathrm{D} / \mathrm{D}_{\max }\right) \cdot 100$, where $\Delta \mathrm{D}$ is the diameter increase from rest in response to $\mathrm{Ca}^{2+}$-free PSS, and $\mathrm{D}_{\max }$ is the maximum diameter measured at the equilibration pressure in $\mathrm{Ca}^{2+-}$ free PSS.

Following equilibration, the dilator reactivity of MCA was assessed in response to increasing concentrations of acetylcholine $\left(10^{-9} \mathrm{M}-10^{-5} \mathrm{M}\right)$. Vascular responses to acetylcholine 
(ACh) were also measured following acute incubation (45-60 minutes) with L-NAME (10-4 M)(results not shown here) and TEMPOL (10-4 M), to assess the contributions of nitric oxide (NO) and oxidative stress, respectively, in modulating vascular reactivity. [21]

Measurement of Vascular NO Bioavailability: From each rat, the abdominal aorta was removed and sectioned into rings, and vascular NO production was assessed using a DAF assay.

Measurement of Cerebral Microvessel Density: Following TTC staining, coronal brain slices (20 um thickness for histology) were flash-frozen in OCT immersed in supercooled isopentane and stored at -80. Samples were prepared with a cryotome (Leica CM3050S; Leica Microsystems) from bregma -1 using a standard Rat Brain Atlas and transferred to charged microscope slides for histological analysis.

Cerebral microvessel and capillary density was evaluated by fluorescence immunohistochemistry using a rat anti-CD31 antibody (BD Biosciences, San Diego, CA) as described preciously [90]. Briefly, brain sections were fixed with $4 \%$ paraformaldehyde, permeabilized with $0.2 \%$ Triton $\mathrm{X}-100$, and blocked with block solution (5\% BSA in PBS) and then incubated with anti-CD31 (1:100) for $1 \mathrm{~h}$ at room temperature in a humidified chamber. Following three washes with PBS, cells were incubated with Alexa Fluor 555-conjugated anti-mouse IgG (1:1000; Invitrogen) for 1 $\mathrm{h}$ at room temperature in a humidified chamber. After a final wash with PBS, cover glass was placed on mounting medium with DAPI (Vector Laboratories, Burlingame, CA) on a slide. Negative control was performed with the same procedure without primary antibody. Images were obtained with an epifluorescence microscope and a SPOT RT camera (Diagnostic Instruments). The microvessels density was analyzed in 6 region of interest (3 in cortex and 3 in striatum) in the MCA territory using ImageJ, as described previously [90]. 
Data and Statistical Analyses: Mechanical responses following challenge with logarithmically increasing dosages of acetylcholine were fit with the three-parameter logistic equation:

$$
y=\min +\left[\frac{\max -\min }{1+10^{\log E D_{50}-x}}\right]
$$

where $y$ represents the vessel diameter, "min" and "max" represent the lower (minimum) and upper (maximum) bounds, respectively, of the change in diameter with agonist concentration, $x$ is the logarithm of the agonist concentration, and $\log \mathrm{ED}_{50}$ represents the logarithm of the agonist concentration $(x)$ where the response $(y)$ is halfway between the bounds. For statistical evaluation of results, we have focused on the changes in the upper bounds as a representation of vessel reactivity, as lower bound remained consistent between all groups (defined as the prechallenge diameter); we did not determine a consistent or significant change to the $\log \mathrm{ED}_{50}$ values between treatment groups. All other statistical analyses utilized Student's t-test or analysis of variance (ANOVA) with Student-Newman-Keuls test post-hoc. Statistical significance is represented by $\mathrm{p}<0.05$.

\section{RESULTS}

Figure 1 presents representative brain slices from each experimental group following staining with TTC. Cortical and striatal areas in the perfusion territory of the right MCA that are stained white in the TTC stain represent the ischemic core of the stroke. The area of neuronal tissue immediately surrounding the ischemic core is characterized as the penumbra. The average total percentage of the right hemisphere stained white during the TTC stain is presented in Figure 1H. LZR showed significantly lower average TTC infarct volume at $24 \mathrm{hrs}$ compared to OZR; at 15 days post stroke, very little or no TTC stained infarct volume was detected in LZR, while 
significant infarcts were measured in OZR. In OZR treated with gp91ds-tat, no TTC infarct volume was measured at 15 days post-stroke.

Figure 2 presents the laser Doppler Flowmetrey (LDF) and mortality data for all animals. To be included in this study as a stroke, cerebral blood flow as measured by LDF must have decreased by at least $70 \%$; the animals from all three groups averaged $80 \%$ reduction of blood flow (Figure 2A). LZR and OZR from both time course groups are reported as one metric, as all LDF recordings occurred immediately following tMCAO. Reperfusion of cerebral blood flow compared to baseline was measured by LDF immediately following withdrawal of the monofilament (Fig 2B). LZR recovered on average 95\% of cerebral blood flow, with a range of $75-130 \%$ of baseline flow, while OZR only recovered on average $\sim 80 \%$ of cerebral blood flow, with a range of $50-90 \%$ of baseline, and two high/two low outlier points. OZR treated with gp91ds-tat, in contrast, all recovered $\sim 95 \%$ of cerebral blood flow, comparable to LZR animals. Trends in recovery of cerebral blood flow collated with TTC infarct volume size $(p=.0038)$ (Figure 2C). Of all groups, only non-treated OZR animals died as a complicated after tMCAO. Survival was $100 \%$ with exception of OZR, which had a mortality rate of $45 \%$ (Figure 2D). Due to variability in time of death, mortality from both the 24 hour and 15 day treatment groups are presented; of note, while data from these animals was included in analysis of cerebral blood flow and infarct size, they were not included in analysis of vascular reactivity to avoid confounding data points with respect to vascular dysfunction developing over time.

Figure 3 presents vasodilator reactivity to ACh in isolated IL and CL MCAs from LZR and OZR at 24 hours and 15 days post stroke. In LZR, at 24 hours post-stroke, dilation to ACh was significantly reduced ( $\sim 50 \%$ reduction to LZR sham MCA) in both IL and CL MCA (Figure 3A). At 15 days post-stroke, reactivity in IL MCA continued to significantly worsen, while 
reactivity in CL-MCA was unchanged compared to reactivity at 24 hours (Figure 3B). Dilator reactivity was reduced about $50 \%$ in OZR sham MCA compared to LZR sham MCA (Figure $3 \mathrm{C})$; this is consistent with our previously published data for vascular dysfunction in 17 week old OZR (Brooks et al). At 24 hours, the CL MCA from OZR was not significantly different from OZR sham MCA, while dilator reactivity in the IL MCA was reduced an additional $50 \%$ compared to CL and sham MCA (Figure 3C). By 15 days post-stroke, OZR CL MCA reactivity had decreased 30\% compared to OZR sham MCA, while dilator reactivity to ACh in IL MCA was completely ablated (Figure 3D).

Figure 4 presents vasodilator reactivity of MCAs from LZR and OZR at 24 hours poststroke, and in gp91ds-tat-treated OZR, following incubation with the reactive oxygen species scavenger TEMPOL. In LZR, reactivity in neither sham MCA, nor IL or CL MCA, significantly changed in the presence of TEMPOL (Figure 4A, 4B). In contrast, sham, IL and CL MCA from OZR all showed significant improvement in dilator reactivity to ACh after treatment with TEMPOL, as both CL-MCA and sham MCA averaged $~ 40 \%$ increase in dilation (Figure 4C). TEMPOL treatment on IL MCA from OZR at 24 hours showed a significant increase as well, returning reactivity to levels comparable to untreated sham MCA (Figure 4D). At 15 days poststroke, IL MCA of OZR, TEMPOL treatment was able to partially rescue dilator reactivity to ACh (Figure 4E); CL MCA of 15 day animals showed no significant difference to TEMPOL treatment (Figure 4F). In OZR treated with gp91ds-tat, reactivity was significantly improved in IL MCA, and trended towards improvement in CL MCA, following incubation with TEMPOL (Figure 4E, 4F).

'Figure 5 presents dilator reactivity of IL and CL MCA from OZRs treated with gp91dstat prior during tMCAO, at 15 days following post-stroke. Dilation to ACh at 15 days in IL MCA 
was significantly improved, increasing from completely non-reactive to levels comparable to 24 hour IL MCA in OZR (Figure 5A). In CL MCA, a small non-significant increase was observed in dilator reactivity compared to 15 day untreated CL MCA (Figure 5B). Changes in maximal dilation were most pronounced at 15 days in IL MCA from OZR treated with gp91ds-tat. This improvement was similar to that observed in other groups following treatment with TEMPOL (Figure 4), suggesting a ROS-mediated mechanism may be partly responsible.

Figure 6 presents NO bioavailability data from isolated aortic ring segments. NO was measured by DAF assay and reported in arbitrary units of fluorescence (AU). At baseline levels of NO production, OZR 15 days post-stroke were the only group that showed a significant decrease. Stimulated production of $\mathrm{NO}$ with methacholine $(\mathrm{MCh})$ showed significant increases in NO production in LZR sham animals only (Figure 6). NO bioavailability remained significantly lower in 15 day post-stroke OZR for all treatments, including baseline and both stimulated MCh treatments, while NO bioavailability in OZR 24 hour post-stroke remained comparable to LZR 24 hour and 15 day post-stroke.

Figure 7 presents cerebral microvessel density measured from the ischemic core and penumbra of LZR and OZR at 15 days post-stroke. Microvessel density was consistent in the contralateral hemispheres between LZR and OZR, and both groups experienced a similar, significant decrease in microvessel density within the ischemic core following stroke (Figure 7). Only in OZR, however, was there also a significant decrease in microvessel density measured in the penumbra at 15 days post stroke. Contralateral brain regions were selected for structural homology with the each brain's designated ischemic core and penumbra areas. 


\section{DISCUSSION}

We present here for the first time detailed descriptions of the progressive nature of cerebrovascular dysfunction in the major resistance arteries of both hemispheres following ischemic stroke in a rodent model of MetSyn, as well as novel findings regarding vasculoprotective and neuroprotective benefits of acute treatment with the selective Nox2 inhibitor gp91ds-tat during ischemic stroke. Cerebrovascular dysfunction in MetSyn impairs regulation of cerebral blood flow, and we demonstrate here that ischemic stroke induces significant impairments in vasodilatory reactivity of MCAs, the major conduits most commonly implicated in ischemic stroke. We further report impairment in MCA vasoreactivity in both LZR and OZR, but found that comorbid presence of MetSyn in OZR increased stroke severity and drove progressive decline of cerebrovascular reactivity following ischemic stroke. Additionally, while oxidative stress has long been considered a major pathological influence on ischemic stroke outcomes, we show that acutely inhibiting Nox2 during the ischemia-reperfusion injury period is sufficient to improve post-stroke vascular reactivity and reduce infarct size in OZR.

Our results are consistent with the theory that ischemic stroke causes severe attenuation of NO bioavailability and cerebrovascular reactivity in the cerebrovasculature following ischemic stroke $[27,28,73-75]$. We show that vasoreactivity is impaired in both the IL and CL hemispheres of LZR and OZR at $24 \mathrm{hrs}$ post-stroke, with reactivity being poorest in IL MCA of OZR. Furthermore, we show a progressive loss of reactivity from 24 hours to 15 days that was most significant in IL MCA of OZR. OZR IL MCA completely lost all vasodilator reactivity to $\mathrm{ACh}$, and indeed became slightly vasoconstrictive to $\mathrm{ACh}$, by 15 days post-stroke. While TEMPOL was able to rescue vasodilatory function at $24 \mathrm{hrs}$, it was ineffective at improving dilation at 15 days in the CL MA of OZR; this suggests that acute oxidative injury during 
ischemic stroke in OXR may lead to more severe impairments in endothelial function. Vasoconstriction of resistance arteries to ACh is consistent with severe injury to the vascular endothelium [76], indicating a progression of endothelial dysfunction stemming from ischemic stroke. While not shown in this paper, we also have data suggesting that myogenic tone and constriction to 5-hydoxytryptamine are increased in MCA of OZR, but not LZR, following ischemic stroke. These data support that pre-existing cerebrovascular dysfunction inherent to MetSyn indeed influences an exacerbation of vascular impairments following ischemic stroke.

Superoxide and other ROS are known to cause vascular and neurological impairments during ischemic stroke. While ROS such as superoxide can directly react with, and neutralize, NO, they can also uncouple eNOS from BH4 and lead to eNOS generation of additional ROS $[38,54,77]$. Previous work from our laboratory has shown a significant, systemic reduction in NO bioavailability in the peripheral and central circulation in MetSyn $[19,22,78-81]$. Here, we show that systemic NO bioavailability is further reduced in LZR and OZR at 15 days following ischemic stroke, indicating that oxidative injury sustained during this event can have long-lasting effects on endothelial dysfunction. Beyond limiting NO bioavailability, ROS can further impair myogenic reactivity $[21,75,82,83]$ and can lead to endothelial cell death and compromise of the cerebral blood brain barrier [7, 61, 84, 85]. Additionally, elevated levels of ROS during stroke are implicated in exacerbating neuronal cell death and oxidative injury, leading to neuronal apoptosis in the ischemic core and penumbra and increasing glutamate-induced excitotoxicity within the brain $[7,29,43,84-86]$. Limiting levels of ROS during stroke has been suggested to be neuroprotective $[68,70,72,87]$ and several studies have sought to identify the most prominent sources of ROS during cerebral ischemia $[53,55,59,60]$. 
Our results suggest that the $\mathrm{NAD}(\mathrm{P}) \mathrm{H}$ oxidase isoform Nox2 is a major source of ROS during ischemic stroke. Nox2 is highly expressed in the cerebrovascular endothelium of both pial and parenchymal vessels [50,57-59], as well as in activated neutrophils and macrophages [58], which are amongst the first immune cells to respond to ischemic injury in the brain [22, 45]. By inhibiting Nox2 during ischemia-reperfusion in OZR with a one-time infusion of the Nox2selective peptide gp91ds-tat, we were able to significantly improve vascular reactivity of the IL MCA at 15 days post-stroke. Of interest, we observed a significantly larger impairment of vasoreactivity in the IL MCA vs CL MCA at 15 days post stroke in OZR, and correspondingly saw improvement only in the IL MCA, and not CL MCA, of OZR treated with gp91ds-tat. One potential explanation for these observations is that oxidative injury is increased in the IL MCA due to the localized incident of ischemia-reperfusion, while oxidative injury in the CL MCA is a reflection of circulating oxidative and inflammatory factors. Hypoxia-induced activity of Nox2 in the cerebrovascular endothelium of IL MCA, and/or activation of Nox2 in the neutrophils and macrophages that migrate to the location of stroke injury, could account for why acute inhibition of Nox2 during stroke was not more protective throughout the entire cerebrovasculature. Accordingly, chronic treatment with gp91ds-tat or with a broad spectrum NAD(P)H oxidase inhibitor such as apocynin prior to, during, or after ischemic stroke may indeed result in improved cerebrovascular reactivity throughout the brain. Additionally, Nox2 inhibition during stroke resulted in a significant reduction of infarct size in OZR at 15 days post-stroke. This finding is consistent with studies showing that antioxidant neuroprotective agents can reduce infarct size in preclinical models of stroke (source) and further suggests that inhibition of Nox2 during the acute stroke injury may help to limit the damage occurring during ischemia and subsequent reperfusion in human patients. Our results suggest that one enzyme, Nox2, could be 
an effective target for improving both cerebrovascular and neurological outcomes during ischemic stroke.

Lastly, we show that ischemic stroke reduces microvascular density in ischemic core 15 days post-stroke in both LZR and OZR. This finding is consistent with previous studies showing impaired neovascularization following stroke in a model of non-obese diabetes [29, 31, 88, 89]. We have previously reported that cortical rarefaction in MetSyn is associated with insulin resistance and loss of NO bioavailability [23]; results of the present study support that loss of NO bioavailability due to oxidative injury could indeed explain vessel rarefaction observed in the ischemic core. The aforementioned study of Fagan et al also found that treatment with metformin is sufficient to restore neovascularization after stroke, suggesting that regulation of plasma glucose may also play an important role [29]. Significantly, we also report rarefaction of cerebral microvessels in the penumbra of OZR, but not LZR, at 15 days following stroke. This novel finding may be the result of increased cerebrovascular dysfunction and oxidative injury during ischemic stroke; poor collateral flow and increased ROS production could lead to expansion of the penumbra and influence a cerebrovascular microenvironment conducive to rarefaction of small vessels in the affected tissue.

\section{Clinical Implications}

Impaired regulation of cerebral blood flow during ischemic stroke is widely recognized as among the most important pathophysiological drivers of neuronal death and stroke severity $[25,26,71]$. While the primary ischemic injury during stroke occurs due to physical blockage of blood flow, neuronal and vascular injuries are greatly exacerbated by oxidative stress during periods of hypoxia. Additionally, several studies have shown that additional injury and neuronal 
death can occur after dissolution of the clot during reperfusion $[44,52,75]$, indicating that treatments could be administered during an active stroke to improve outcomes beyond simple restoration of blood flow. Often, the vascular health of the individual, or indeed the preclinical model, is overlooked when considering therapies for ischemic stroke. Cerebrovascular dysfunction is hallmark of MetSyn and other types of cardiovascular disease, and is itself an important risk factor for developing stroke; therefore, it is important to factor in pre-existing mechanisms of cerebrovascular dysfunction when studying the pathological outcomes of ischemic stroke or evaluating novel therapeutics. To this end, we conducted experimental strokes in OZR, a well-established model of MetSyn and cerebrovascular disease, and would encourage other groups to consider a similar approach.

Reducing oxidative injury during ischemia-reperfusion has been proposed by several groups as an important mechanism for improving outcomes of ischemic stroke. In this study, we evaluated specific targeting of Nox2 during ischemia-reperfusion injury (as opposed to chronic pre- or post- treatment with antioxidants) to assess potential benefits of reducing ROS during the stroke itself. Our initial findings are encouraging, as we demonstrated that this strategy was sufficient to prevent severe cerebrovascular dysfunction in the IL MCA and to reduce infarct size and mortality. While we did not improve reactivity in CL MCA, this may be due to the acute nature of our treatment period; the only injury we prevented was the acute injury from ischemiareperfusion, and not injury due to chronic oxidative and inflammatory conditions that persist from MetSyn and ischemic stroke. Nevertheless, we report here that inhibition of Nox2 during stroke may be a viable therapeutic pathway for improving outcomes to ischemic stroke. Our future aims will include evaluating how chronic reduction of ROS following ischemia- 
reperfusion, either with gp91ds-tat or a more broad antioxidant, improves cerebrovascular reactivity and neurological outcomes.

\section{Limitations of present study:}

This study is limited by the small sample size, due to its nature as a pilot study. Further extrapolation of the results summarized here is therefore discouraged, and this study needs to be completed in full with adequate animal numbers to confer statistical power. Another limitation of this study is the lack of a dose-response experiment to determine ideal dosage of gp91ds-tat; a high dose was selected based on the literature, but tolerance and efficacy of this drug in OZR needs to be more fully established. Lastly, this study reflected only 1 time point for administration of gp91ds-tat, and only two time outcome points; an expanded study looking at the effects of pre-stroke and post-stroke administration, as well as chronic vs acute dosing, is needed for this study. Comparison with a more broad Nox inhibitor, such as apocynin, may be warranted as well.

\section{Acknowledgements:}

The authors would like to thank WVU Experimental Stroke Core and Animal Care facilities for excellent technical support and animal monitoring during these procedures, as well as Kent Lemaster, Ashlee Seldomridge, and Whitney Sheets for technical support. Additionally, we also acknowledge the support provided through Center for Cardiovascular and Respiratory Sciences and the Clinical and Translational Sciences Institute at the West Virginia University Health Sciences Center 


\section{Grants}

This study was supported by the American Heart Association grants IRG 14330015, PRE 16850005, EIA 0740129N and pre-doctoral fellowship AHA 14PRE 20380386. Research reported in this publication was supported by the National Institute of General Medical Sciences of the National Institutes of Health under Award Number U54GM104942.

\section{Disclaimer}

The content is solely the responsibility of the authors and does not necessarily represent the official views of the National Institutes of Health. 


\section{LITERATURE CITED}

1. Frizzell, J.P., Acute stroke: pathophysiology, diagnosis, and treatment. AACN Clin Issues, 2005. 16(4): p. 421-40; quiz 597-8.

2. Lloyd-Jones, D., et al., Executive summary: heart disease and stroke statistics--2010 update: a report from the American Heart Association. Circulation, 2010. 121(7): p. 948-54.

3. American Stroke Association. Stroke Treatments. 2013 [cited 20165 May]; Available from: http://www.strokeassociation.org/STROKEORG/AboutStroke/Treatment/Stroke-

Treatments_UCM_310892_Article.jsp\#.V0VUMOT3Ohm.

4. Writing Group, M., et al., Heart Disease and Stroke Statistics-2016 Update: A Report From the American Heart Association. Circulation, 2016. 133(4): p. e38-60.

5. Seshadri, S., et al., The lifetime risk of stroke: estimates from the Framingham Study. Stroke, 2006. 37(2): p. 345-50.

6. Chapman, S.N., et al., Current perspectives on the use of intravenous recombinant tissue plasminogen activator (tPA) for treatment of acute ischemic stroke. Vasc Health Risk Manag, 2014. 10: p. 75-87.

7. Deb, P., S. Sharma, and K.M. Hassan, Pathophysiologic mechanisms of acute ischemic stroke: An overview with emphasis on therapeutic significance beyond thrombolysis. Pathophysiology, 2010. 17(3): p. 197-218.

8. Schwamm, L.H., et al., Temporal trends in patient characteristics and treatment with intravenous thrombolysis among acute ischemic stroke patients at Get With The GuidelinesStroke hospitals. Circ Cardiovasc Qual Outcomes, 2013. 6(5): p. 543-9.

9. CDC.gov Prevalence of Stroke- United States, 2006-2010. 2012. 379-382.

10. Brown, W.V., Metabolic syndrome and risk of stroke. Clin Cornerstone, 2004. 6 Suppl 3: p. S30-4.

11. Chin, J.H. and N. Vora, The global burden of neurologic diseases. Neurology, 2014. 83(4): p. $349-51$.

12. Good, D.C., Cerebrovascular Disease, in Clinical Methods: The History, Physical, and Laboratory Examinations, H.K. Walker, W.D. Hall, and J.W. Hurst, Editors. 1990: Boston.

13. National Health Service. Cerebrovascular Disease. 2015 [cited 20165 May]; Available from: http://www.nhs.uk/conditions/cerebrovascular-disease/pages/definition.aspx. 
14. Prevention, C.f.D.C.a. Stroke. 2016 [cited 20164 April]; Available from: http://www.cdc.gov/stroke/index.htm.

15. American Heart Association. Metabolic Syndrome. 2014 [cited $20163 \mathrm{March}$ ]; Available from: http://www.heart.org/HEARTORG/Conditions/More/MetabolicSyndrome/MetabolicSyndrome_UCM_002080_SubHomePage.jsp.

16. Aguilar, M., et al., Prevalence of the metabolic syndrome in the United States, 2003-2012. JAMA, 2015. 313(19): p. 1973-4.

17. Boden-Albala, B., et al., Metabolic syndrome and ischemic stroke risk: Northern Manhattan Study. Stroke, 2008. 39(1): p. 30-5.

18. Aleixandre de Artinano, A. and M. Miguel Castro, Experimental rat models to study the metabolic syndrome. Br J Nutr, 2009. 102(9): p. 1246-53.

19. Frisbee, J.C. and M.D. Delp, Vascular function in the metabolic syndrome and the effects on skeletal muscle perfusion: lessons from the obese Zucker rat. Essays Biochem, 2006. 42: p. 14561.

20. Tofovic, S.P. and E.K. Jackson, Rat models of the metabolic syndrome. Methods Mol Med, 2003. 86: p. 29-46.

21. Butcher, J.T., et al., Differential impact of dilator stimuli on increased myogenic activation of cerebral and skeletal muscle resistance arterioles in obese zucker rats. Microcirculation, 2013. 20(7): p. 579-89.

22. Brooks, S.D., et al., Metabolic syndrome impairs reactivity and wall mechanics of cerebral resistance arteries in obese Zucker rats. Am J Physiol Heart Circ Physiol, 2015. 309(11): p. H1846-59.

23. Chantler, P.D., et al., Cerebral Cortical Microvascular Rarefaction in Metabolic Syndrome is Dependent on Insulin Resistance and Loss of Nitric Oxide Bioavailability. Microcirculation, 2015. 22(6): p. 435-45.

24. Ergul, A., et al., Cerebrovascular complications of diabetes: focus on stroke. Endocr Metab Immune Disord Drug Targets, 2012. 12(2): p. 148-58.

25. Faraci, F.M., Protecting against vascular disease in brain. Am J Physiol Heart Circ Physiol, 2011. 300(5): p. H1566-82.

26. Miller, A.A., K. Budzyn, and C.G. Sobey, Vascular dysfunction in cerebrovascular disease: mechanisms and therapeutic intervention. Clin Sci (Lond), 2010. 119(1): p. 1-17. 
27. Osmond, J.M., et al., Obesity increases blood pressure, cerebral vascular remodeling, and severity of stroke in the Zucker rat. Hypertension, 2009. 53(2): p. 381-6.

28. Osmond, J.M., J.D. Mintz, and D.W. Stepp, Preventing increased blood pressure in the obese Zucker rat improves severity of stroke. Am J Physiol Heart Circ Physiol, 2010. 299(1): p. H55-61.

29. Abdelsaid, M., et al., Metformin treatment in the period after stroke prevents nitrative stress and restores angiogenic signaling in the brain in diabetes. Diabetes, 2015. 64(5): p. 1804-17.

30. Knight, R.A., et al., Acute blood-brain barrier opening in experimentally induced focal cerebral ischemia is preferentially identified by quantitative magnetization transfer imaging. Magn Reson Med, 2005. 54(4): p. 822-32.

31. Prakash, R., et al., Cerebral neovascularization and remodeling patterns in two different models of type 2 diabetes. PLoS One, 2013. 8(2): p. e56264.

32. Cipolla, M.J. and J.A. Godfrey, Effect of hyperglycemia on brain penetrating arterioles and cerebral blood flow before and after ischemia/reperfusion. Transl Stroke Res, 2010. 1(2): p. 12734.

33. Schreurs, M.P. and M.J. Cipolla, Cerebrovascular dysfunction and blood-brain barrier permeability induced by oxidized LDL are prevented by apocynin and magnesium sulfate in female rats. J Cardiovasc Pharmacol, 2014. 63(1): p. 33-9.

34. Erdos, B., et al., Cerebrovascular dysfunction in Zucker obese rats is mediated by oxidative stress and protein kinase C. Diabetes, 2004. 53(5): p. 1352-9.

35. Iadecola, C., Neurovascular regulation in the normal brain and in Alzheimer's disease. Nat Rev Neurosci, 2004. 5(5): p. 347-60.

36. Iadecola, C. and R.L. Davisson, Hypertension and cerebrovascular dysfunction. Cell Metab, 2008. 7(6): p. 476-84.

37. Mayhan, W.G., Impairment of endothelium-dependent dilatation of cerebral arterioles during diabetes mellitus. Am J Physiol, 1989. 256(3 Pt 2): p. H621-5.

38. Schwaninger, R.M., H. Sun, and W.G. Mayhan, Impaired nitric oxide synthase-dependent dilatation of cerebral arterioles in type II diabetic rats. Life Sci, 2003. 73(26): p. 3415-25.

39. Trauernicht, A.K., et al., Enalapril prevents impaired nitric oxide synthase-dependent dilatation of cerebral arterioles in diabetic rats. Stroke, 2003. 34(11): p. 2698-703. 
40. Kitayama, J., et al., Cerebral vascular dysfunction during hypercholesterolemia. Stroke, 2007. 38(7): p. 2136-41.

41. Maneen, M.J. and M.J. Cipolla, Peroxynitrite diminishes myogenic tone in cerebral arteries: role of nitrotyrosine and F-actin. Am J Physiol Heart Circ Physiol, 2007. 292(2): p. H1042-50.

42. Mayhan, W.G., et al., Age-related alterations in reactivity of cerebral arterioles: role of oxidative stress. Microcirculation, 2008. 15(3): p. 225-36.

43. Olmez, I. and H. Ozyurt, Reactive oxygen species and ischemic cerebrovascular disease. Neurochem Int, 2012. 60(2): p. 208-12.

44. Chamorro, A., et al., Neuroprotection in acute stroke: targeting excitotoxicity, oxidative and nitrosative stress, and inflammation. Lancet Neurol, 2016.

45. Iadecola, C. and J. Anrather, The immunology of stroke: from mechanisms to translation. Nat Med, 2011. 17(7): p. 796-808.

46. Smith, C.J., et al., The immune system in stroke: clinical challenges and their translation to experimental research. J Neuroimmune Pharmacol, 2013. 8(4): p. 867-87.

47. Stanimirovic, D. and K. Satoh, Inflammatory mediators of cerebral endothelium: a role in ischemic brain inflammation. Brain Pathol, 2000. 10(1): p. 113-26.

48. Modrick, M.L., et al., Role of oxidative stress and AT1 receptors in cerebral vascular dysfunction with aging. Am J Physiol Heart Circ Physiol, 2009. 296(6): p. H1914-9.

49. Arrick, D.M., et al., Losartan improves impaired nitric oxide synthase-dependent dilatation of cerebral arterioles in type 1 diabetic rats. Brain Res, 2008. 1209: p. 128-35.

50. Lynch, C.M., et al., Nox2-derived superoxide contributes to cerebral vascular dysfunction in diet-induced obesity. Stroke, 2013. 44(11): p. 3195-201.

51. Crack, P.J. and J.M. Taylor, Reactive oxygen species and the modulation of stroke. Free Radic Biol Med, 2005. 38(11): p. 1433-44.

52. Gursoy-Ozdemir, Y., A. Can, and T. Dalkara, Reperfusion-induced oxidative/nitrative injury to neurovascular unit after focal cerebral ischemia. Stroke, 2004. 35(6): p. 1449-53.

53. Chrissobolis, S. and F.M. Faraci, The role of oxidative stress and NADPH oxidase in cerebrovascular disease. Trends Mol Med, 2008. 14(11): p. 495-502.

54. Mayhan, W.G., et al., Inhibition of $N A D(P) H$ oxidase alleviates impaired NOS-dependent responses of pial arterioles in type 1 diabetes mellitus. Microcirculation, 2006. 13(7): p. 567-75. 
55. Miller, A.A., et al., NADPH oxidase activity and function are profoundly greater in cerebral versus systemic arteries. Circ Res, 2005. 97(10): p. 1055-62.

56. Guzik, T.J., et al., Mechanisms of increased vascular superoxide production in human diabetes mellitus: role of $N A D(P) H$ oxidase and endothelial nitric oxide synthase. Circulation, 2002. 105(14): p. 1656-62.

57. Schroder, K., et al., NADPH oxidase Nox2 is required for hypoxia-induced mobilization of endothelial progenitor cells. Circ Res, 2009. 105(6): p. 537-44.

58. Bedard, K. and K.H. Krause, The NOX family of ROS-generating NADPH oxidases: physiology and pathophysiology. Physiol Rev, 2007. 87(1): p. 245-313.

59. Miller, A.A., et al., Augmented superoxide production by Nox2-containing NADPH oxidase causes cerebral artery dysfunction during hypercholesterolemia. Stroke, 2010. 41(4): p. 784-9.

60. Park, L., et al., Nox2-derived reactive oxygen species mediate neurovascular dysregulation in the aging mouse brain. J Cereb Blood Flow Metab, 2007. 27(12): p. 1908-18.

61. Basuroy, S., et al., Nox4 NADPH oxidase mediates oxidative stress and apoptosis caused by TNF-alpha in cerebral vascular endothelial cells. Am J Physiol Cell Physiol, 2009. 296(3): p. C422-32.

62. Kuroda, J., et al., Nox4 is a major source of superoxide production in human brain pericytes. J Vasc Res, 2014. 51(6): p. 429-38.

63. Paravicini, T.M., et al., Increased NADPH-oxidase activity and Nox4 expression during chronic hypertension is associated with enhanced cerebral vasodilatation to NADPH in vivo. Stroke, 2004. 35(2): p. 584-9.

64. Basuroy, S., et al., Nox4 NADPH oxidase-derived reactive oxygen species, via endogenous carbon monoxide, promote survival of brain endothelial cells during TNF-alpha-induced apoptosis. Am J Physiol Cell Physiol, 2011. 300(2): p. C256-65.

65. Nauseef, W.M., Assembly of the phagocyte NADPH oxidase. Histochem Cell Biol, 2004. 122(4): p. 277-91.

66. Brooks, S.D., et al., Admission neutrophil-lymphocyte ratio predicts 90 day outcome after endovascular stroke therapy. J Neurointerv Surg, 2013.

67. Bayramgurler, D., et al., The effect of etanercept on aortic nitric oxide-dependent vasorelaxation in an unpredictable chronic, mild stress model of depression in rats. Eur $\mathbf{J}$ Pharmacol, 2013. 710(1-3): p. 67-72. 
68. Elewa, H.F., et al., Early atorvastatin reduces hemorrhage after acute cerebral ischemia in diabetic rats. J Pharmacol Exp Ther, 2009. 330(2): p. 532-40.

69. Elewa, H.F., et al., Blood pressure lowering after experimental cerebral ischemia provides neurovascular protection. J Hypertens, 2007. 25(4): p. 855-9.

70. Fagan, S.C., et al., Targets for vascular protection after acute ischemic stroke. Stroke, 2004. 35(9): p. 2220-5.

71. Palomares, S.M. and M.J. Cipolla, Vascular Protection Following Cerebral Ischemia and Reperfusion. J Neurol Neurophysiol, 2011. 2011.

72. Arenillas, J.F., M.A. Moro, and A. Davalos, The metabolic syndrome and stroke: potential treatment approaches. Stroke, 2007. 38(7): p. 2196-203.

73. Chan, S.L., et al., Pial Collateral Reactivity During Hypertension and Aging: Understanding the Function of Collaterals for Stroke Therapy. Stroke, 2016. 47(6): p. 1618-25.

74. Cipolla, M.J. and L.V. Bullinger, Reactivity of brain parenchymal arterioles after ischemia and reperfusion. Microcirculation, 2008. 15(6): p. 495-501.

75. Cipolla, M.J., et al., Reperfusion decreases myogenic reactivity and alters middle cerebral artery function after focal cerebral ischemia in rats. Stroke, 1997. 28(1): p. 176-80.

76. Ludmer, P.L., et al., Paradoxical vasoconstriction induced by acetylcholine in atherosclerotic coronary arteries. N Engl J Med, 1986. 315(17): p. 1046-51.

77. Landmesser, U., et al., Oxidation of tetrahydrobiopterin leads to uncoupling of endothelial cell nitric oxide synthase in hypertension. J Clin Invest, 2003. 111(8): p. 1201-9.

78. Frisbee, J.C., Reduced nitric oxide bioavailability contributes to skeletal muscle microvessel rarefaction in the metabolic syndrome. Am J Physiol Regul Integr Comp Physiol, 2005. 289(2): p. R307-R316.

79. Goodwill, A.G., M.E. James, and J.C. Frisbee, Increased vascular thromboxane generation impairs dilation of skeletal muscle arterioles of obese Zucker rats with reduced oxygen tension. Am J Physiol Heart Circ Physiol, 2008. 295(4): p. H1522-8.

80. Phillips, S.A., F.A. Sylvester, and J.C. Frisbee, Oxidant stress and constrictor reactivity impair cerebral artery dilation in obese Zucker rats. Am J Physiol Regul Integr Comp Physiol, 2005. 288(2): p. R522-30. 
81. Stapleton, P.A., et al., Altered mechanisms of endothelium-dependent dilation in skeletal muscle arterioles with genetic hypercholesterolemia. Am J Physiol Regul Integr Comp Physiol, 2007. 293(3): p. R1110-9.

82. Coulson, R.J., et al., Effects of ischemia and myogenic activity on active and passive mechanical properties of rat cerebral arteries. Am J Physiol Heart Circ Physiol, 2002. 283(6): p. H2268-75.

83. Zimmermann, P.A., et al., Increased myogenic tone and diminished responsiveness to ATPsensitive K+ channel openers in cerebral arteries from diabetic rats. Circ Res, 1997. 81(6): p. 996-1004.

84. Ankarcrona, M., et al., Glutamate-Induced Neuronal Death - a Succession of Necrosis or Apoptosis Depending on Mitochondrial-Function. Neuron, 1995. 15(4): p. 961-973.

85. Banasiak, K.J., Y. Xia, and G.G. Haddad, Mechanisms underlying hypoxia-induced neuronal apoptosis. Prog Neurobiol, 2000. 62(3): p. 215-49.

86. Carmody, R.J., A.J. McGowan, and T.G. Cotter, Reactive oxygen species as mediators of photoreceptor apoptosis in vitro. Experimental Cell Research, 1999. 248(2): p. 520-530.

87. Abdelsaid, M., M. Coucha, and A. Ergul, Cerebrovasculoprotective effects of azilsartan medoxomil in diabetes. Transl Res, 2014. 164(5): p. 424-32.

88. Ergul, A., et al., Hyperglycemia, diabetes and stroke: focus on the cerebrovasculature. Vascul Pharmacol, 2009. 51(1): p. 44-9.

89. Li, W., et al., Adaptive cerebral neovascularization in a model of type 2 diabetes: relevance to focal cerebral ischemia. Diabetes, 2010. 59(1): p. 228-35.

90. Zechariah, A., et al., Vascular endothelial growth factor promotes pericyte coverage of brain capillaries, improves cerebral blood flow during subsequent focal cerebral ischemia, and preserves the metabolic penumbra. Stroke, 2013. 44(6): p. 1690-7. 


\section{FIGURE LEGENDS}

Figure 1. TTC stain of coronal brain slices to measure infarct area at $24 \mathrm{hrs}$ or 15 days poststroke. Representative slices are shown for LZR- Sham (A), tMCAO 24 hrs (B), and tMCAO 15 days (E). As well as for OZR- Sham (D), tMCAO 24 hrs (C), and tMCAO 15 days (F), and OZR treated with gp91ds-tat $(\mathrm{G})$. Panel $(\mathrm{H})$ shows a graphical representation of the average infarct size per group. Given small sample size, statistical data not shown.

Figure 2. Trans-cranical Laser Doppler Flowmetrey (LDF) measurements of blood flow during the tMCAO (A), and following reperfusion (B). The $\%$ of flow recovered was plotted against infarct area to determine correlation (C). Mortality rates are presented in panel (D). In panel D, the lines for LZR Sham, OZR Sham, LZR MCAO, and OZR MCAO all overlap at 100\%.

Figure 3. Ex vivo IL and CL middle cerebral artery reactivity in LZR and OZR following ischemic stroke. Panel (A): Dilation to acetylcholine for IL and CL MCA of LZR. 24 hours post stroke. Panel (B): Dilation to acetylcholine for IL and CL MCA of LZR. 15 days post stroke. Panel (C): Dilation to acetylcholine for IL and CL MCA of OZR. 24 hours post stroke. Panel (D): Dilation to acetylcholine for IL and CL MCA of OZR. 15 days post stroke. All data are

presented as mean \pm SE. $* p<0.05$ versus responses in LZR control at 17 weeks; $\uparrow \mathrm{p}<0.05$ versus responses in OZR control at 17 weeks. Please see text for more details.

Figure 4. Ex vivo middle cerebral artery reactivity in LZR and OZR following ischemic stroke. Vessels were incubated with TEMPOL, rinsed thoroughly, and then incubated with L-NAME, 
and assessed for reactivity to acetylcholine. Panel (A): Dilation to acetylcholine for LZR IL MCA at 24 hours post stroke. Panel (B): Dilation to acetylcholine for LZR CL MCA at 24 hours post stroke. Panel (C): Dilation to acetylcholine for OZR IL MCA at 24 hours post stroke. Panel (D): Dilation to acetylcholine for OZR CL MCA at 24 hours post stroke. Panel (E): Dilation to acetylcholine for OZR IL MCA, treated with gp91ds-tat, at 24 hours post stroke. Panel (F): Dilation to acetylcholine for OZR CL MCA, treated with gp91ds-tat, at 24 hours post stroke. All data are presented as mean $\pm \mathrm{SE}$. $* \mathrm{p}<0.05$ versus responses in LZR control at 17 weeks; $\uparrow$ $\mathrm{p}<0.05$ versus responses in OZR control at 17 weeks. Please see text for more details.

Figure 5. Ex vivo middle cerebral artery reactivity for IL and CL MCA of OZR treated with gp91ds-tat during tMCAO. Panel (A): Dilation to acetylcholine, comparing IL MCA of treated and untreated OZR at 15 days post stroke. Panel (B): Dilation to acetylcholine, comparing CL MCA of treated and untreated OZR at 15 days post stroke. Panel (C): Bar graph of maximum dilation to acetylcholine $\left[10^{-5}\right] \mathrm{M}$ for all time and treatment groups. All data are presented as mean \pm SE. $* \mathrm{p}<0.05$ versus responses in LZR control at 17 weeks; $\uparrow \mathrm{p}<0.05$ versus responses in OZR control at 17 weeks. Please see text for more details.

Figure 6. Arbitrary fluorescence units representing nitric oxide produced from aortic ring segments of LZR and OZR, at 24 hrs or 15 days post stroke. NO was measured at unstimulated baseline, and after stimulation with Methacholine $\left[10^{-5}\right] \mathrm{M}$ and $\left[10^{-4}\right] \mathrm{M}$. Fluorescence was measured by DAF immunoassay. Due to small sample size, statistical analysis is not shown. 
Figure 7. Cortical and striatal measurements of microvessel density in the cortex and striatum of the IL and CL MCA perfusion territories, taken 15 days post stroke. Microvessel density was measured by staining arterioles and capillaries with anti-CD31 antibody. While a strong trend is exhibited in this data, due to small sample size, statistical data is not shown. 

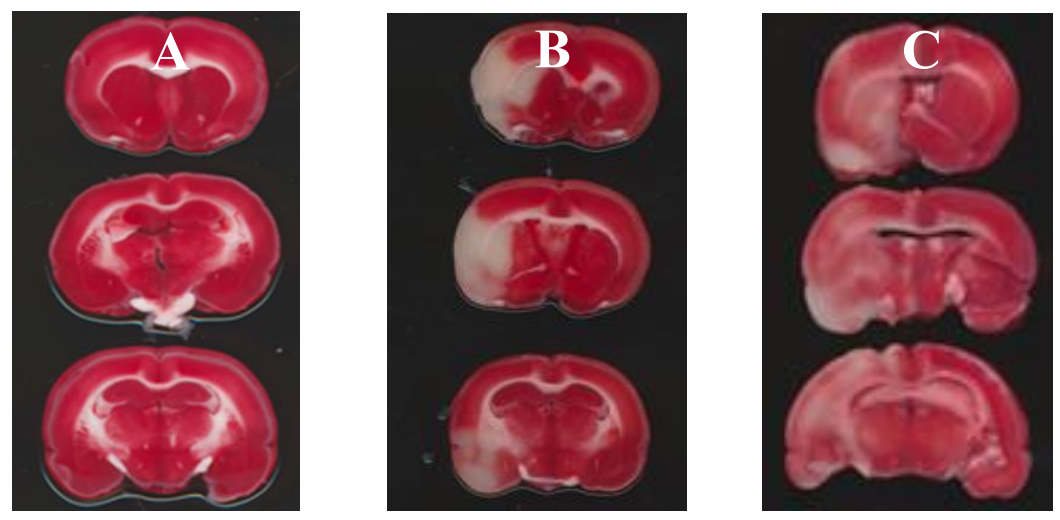

LZR-Sham

LZR-tMCAO

OZR-tMCAO

24 Hours

24 Hours
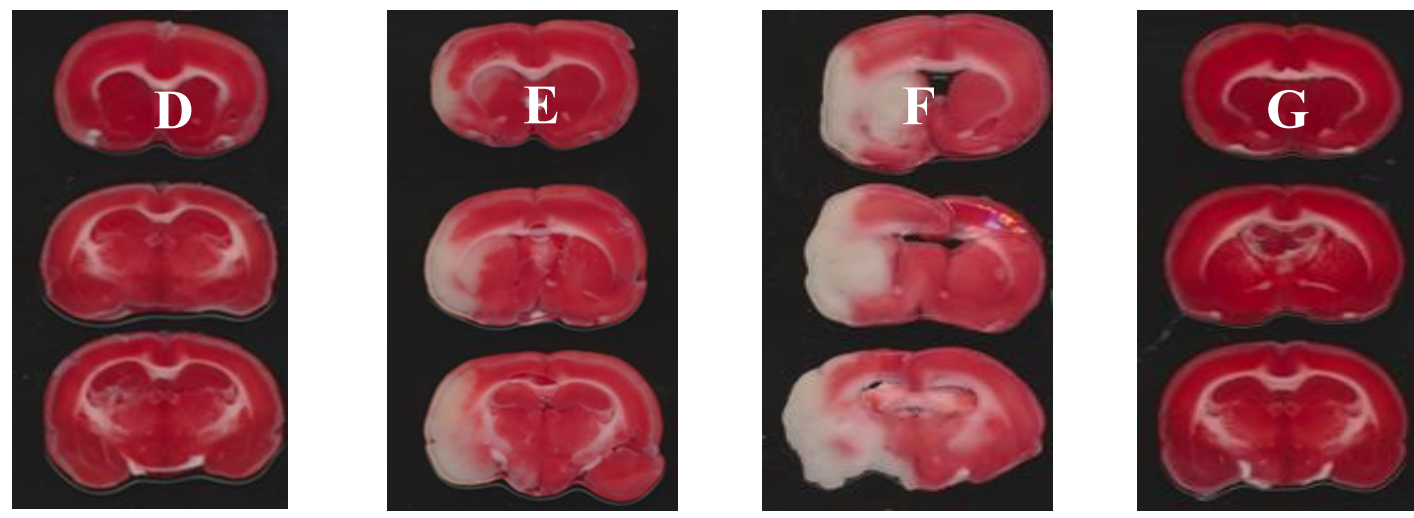

OZR-Sham

LZR-tMCAO

15 Days
OZR-tMCAO
15 Days
OZR-tMCAO

+gp91ds-tat

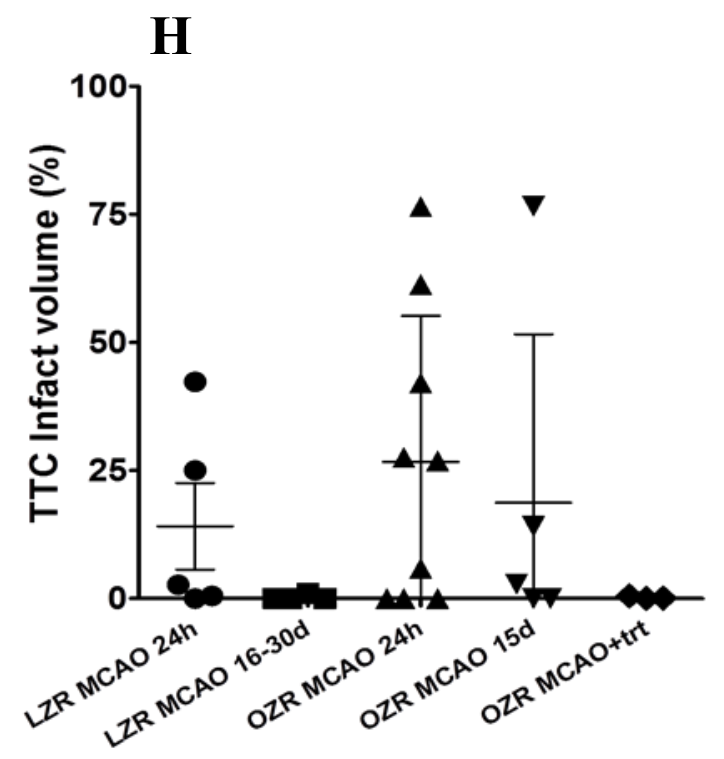

Figure 1 (TTC Staining)

Brooks et al 

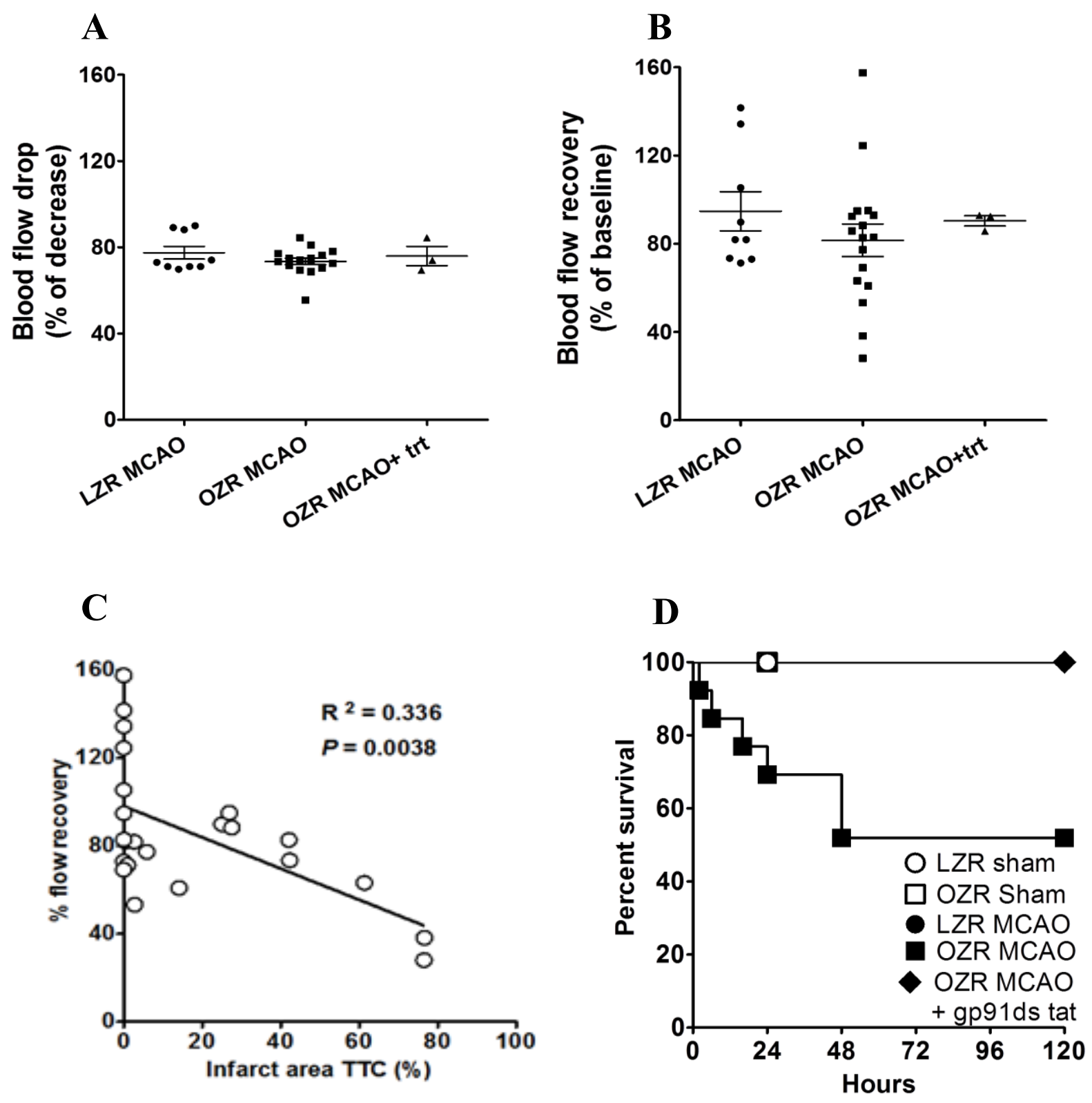

Figure 2 (Cerebral Blood Flow and Mortality)

Brooks et al 

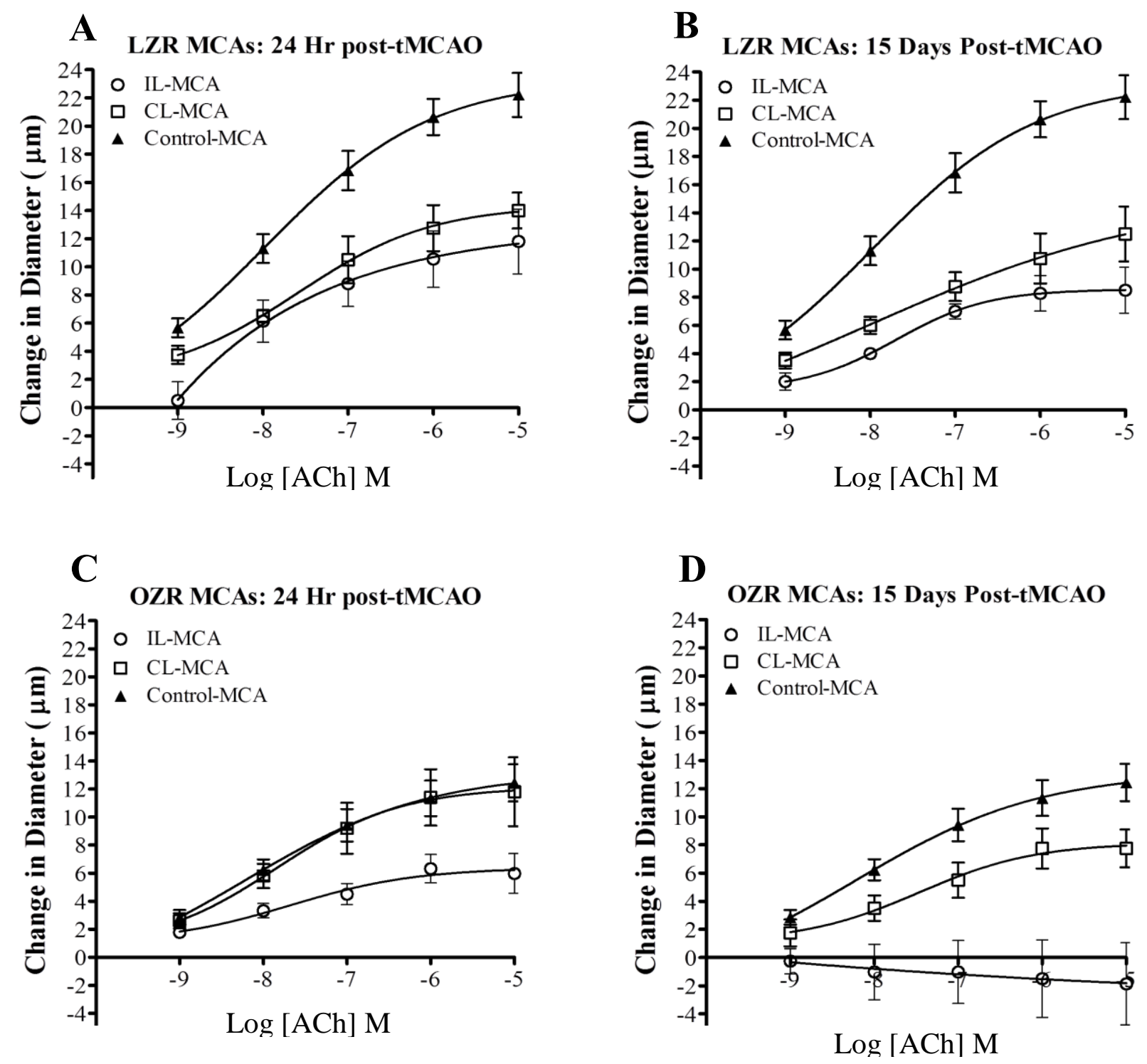

Figure 3 (MCA Dilator Reactivity)

Brooks et al 
A

IL-MCA: LZR 24 Hours Post-tMCAO

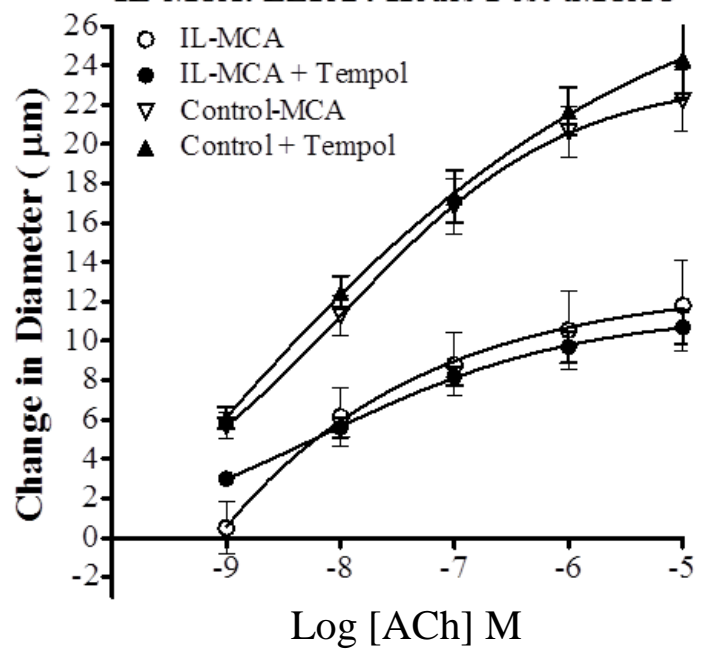

C

\section{IL-MCA: OZR 24 Hours Post-tMCAO}

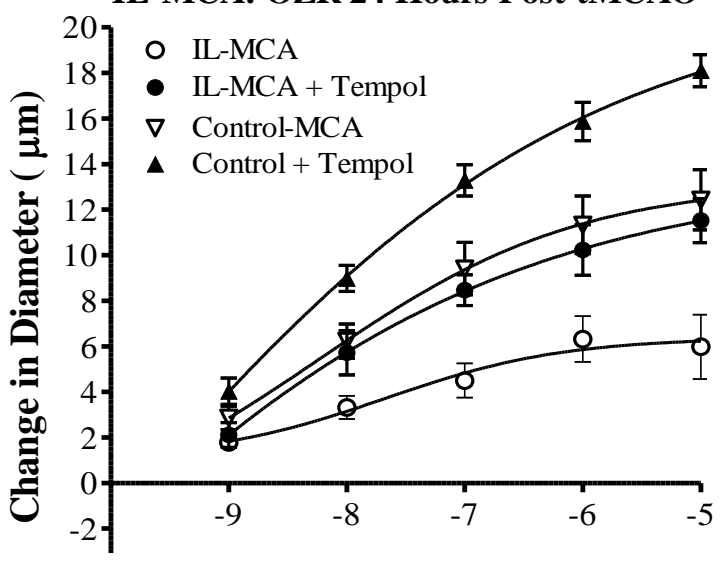

$\log [\mathrm{ACh}] \mathrm{M}$

E

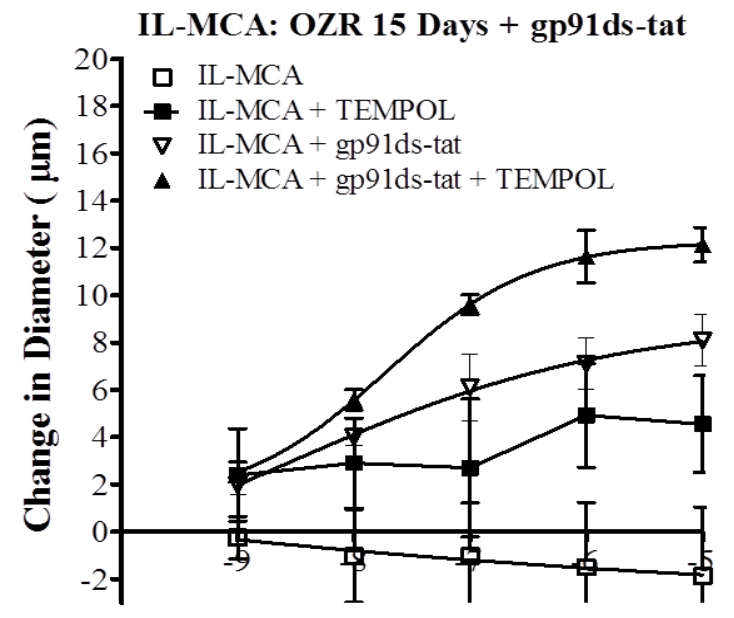

\section{B}

CL-MCA: LZR 24 Hours Post-tMCAO

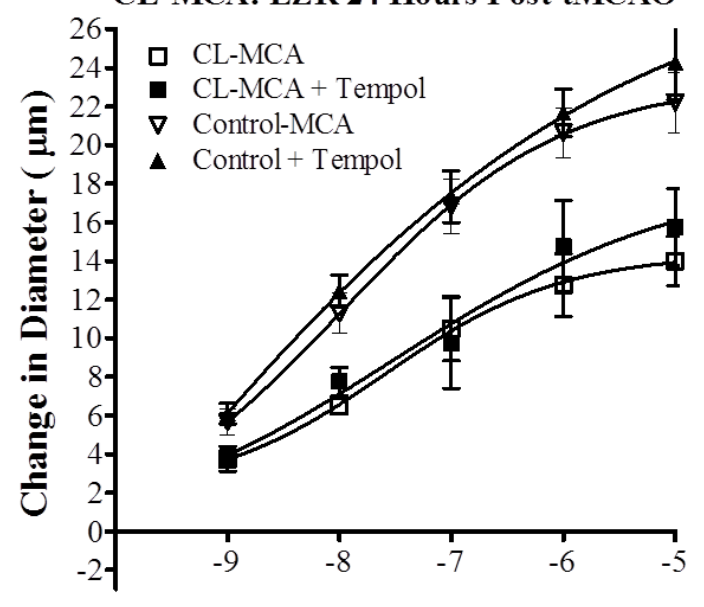

D

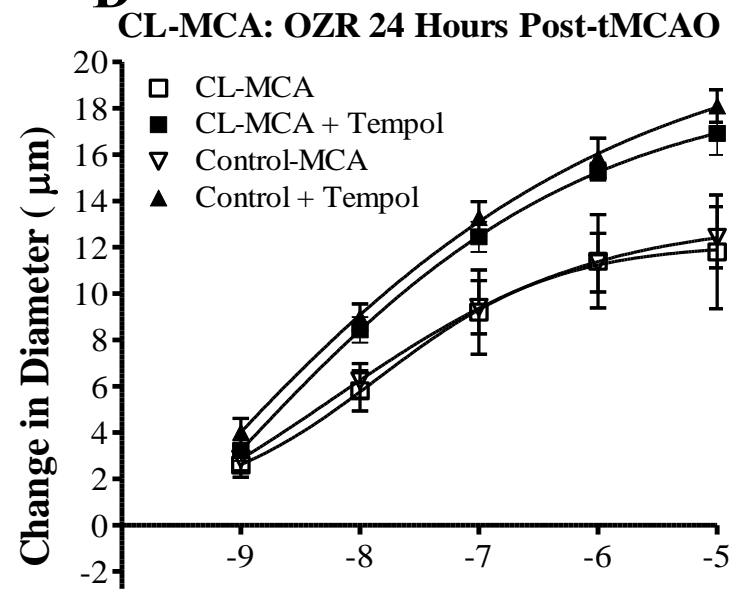

$\log [\mathrm{ACh}] \mathrm{M}$

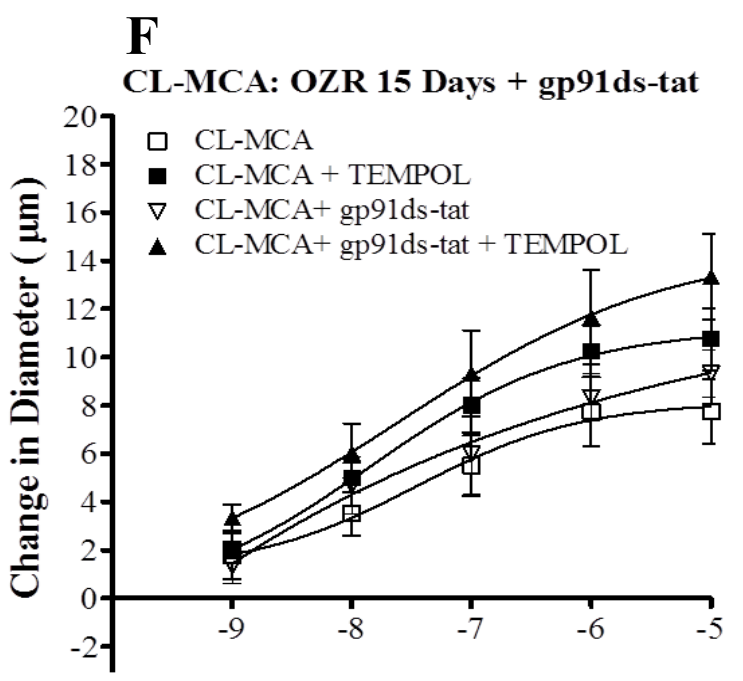

Figure 4 (Effect of TEMPOL on MCA Dilation)

Brooks et al 

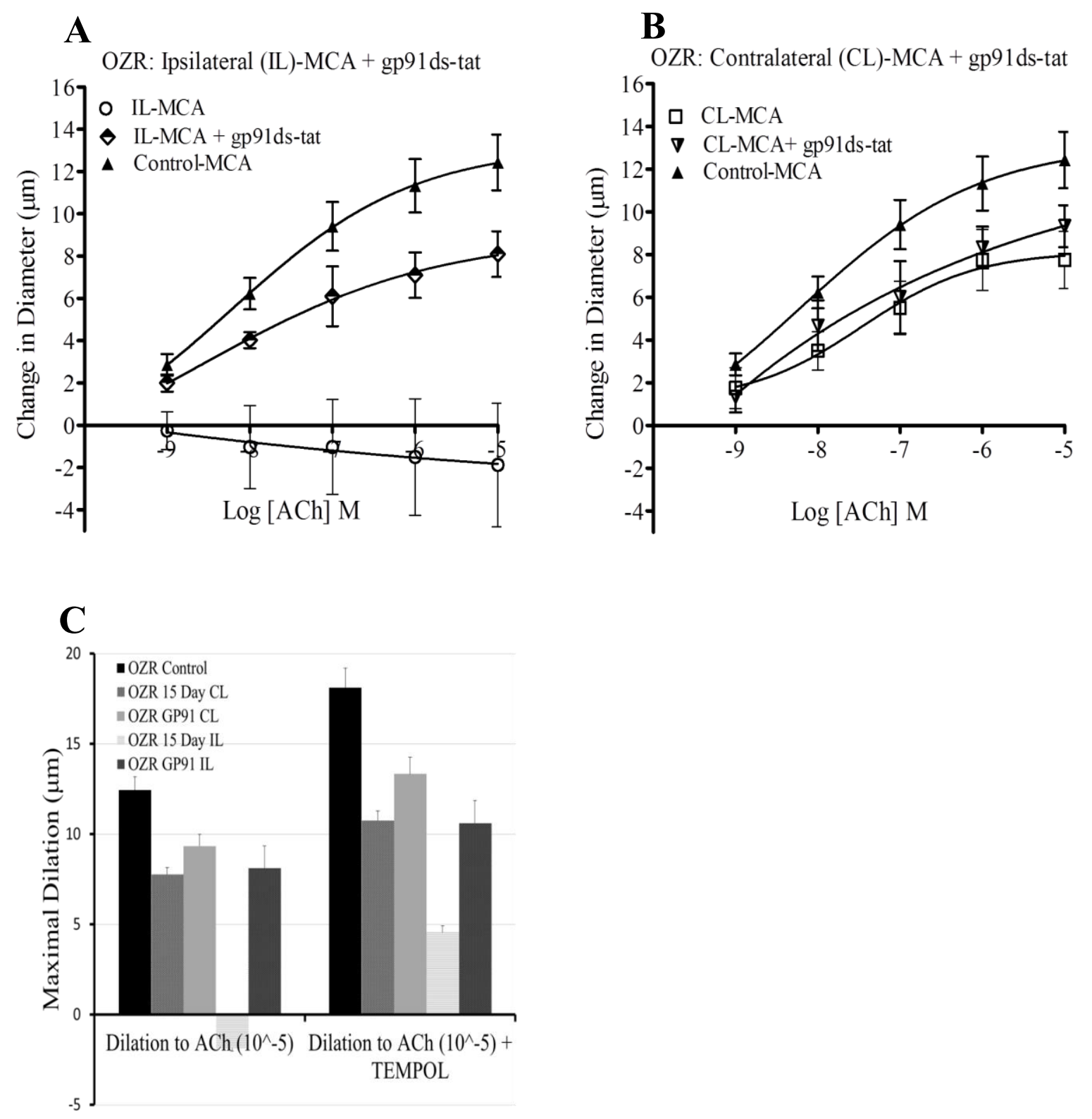

Figure 5 (MCA from gp91ds-tat treated OZR)

\section{Brooks et al}




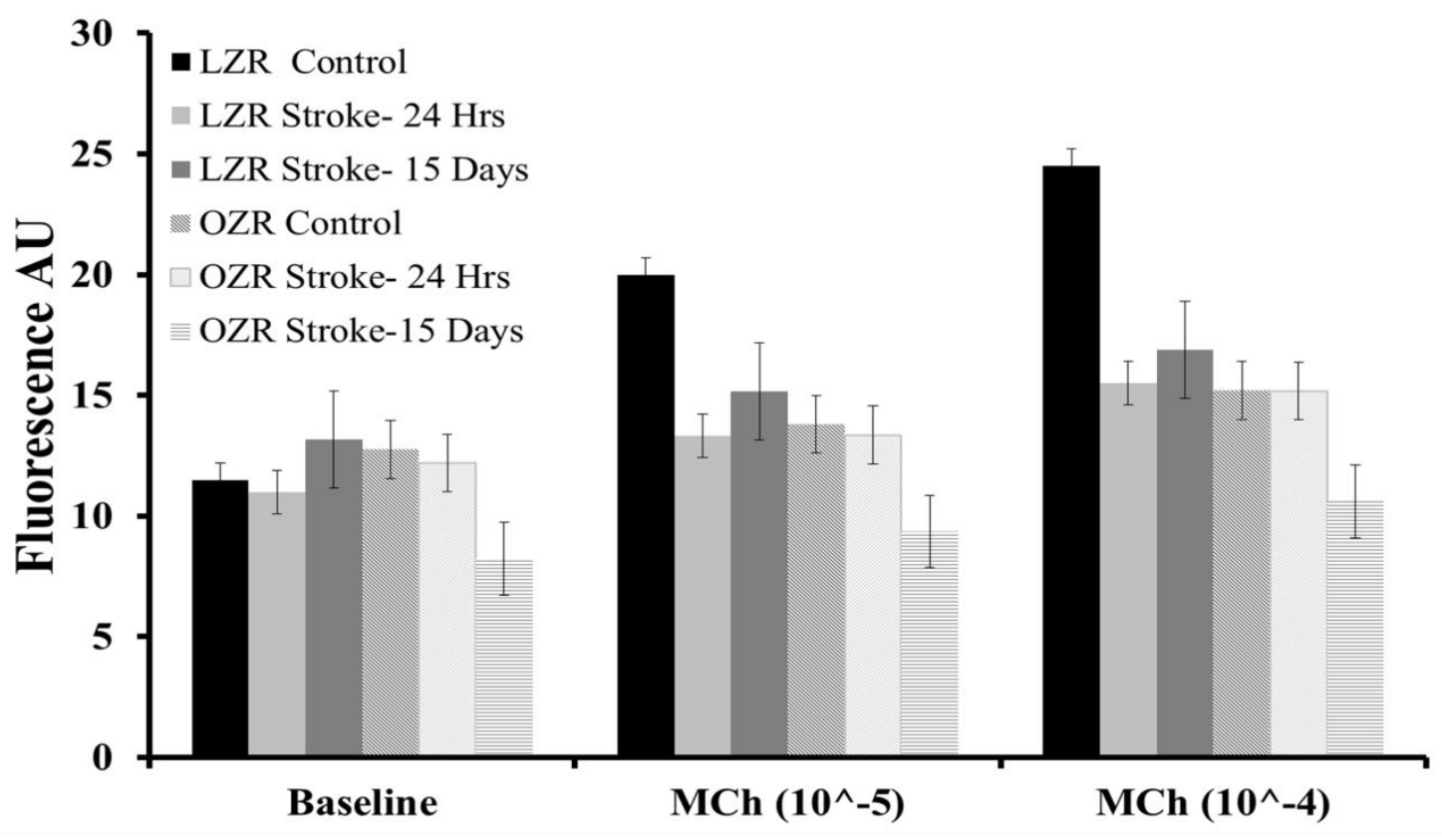

Figure 6 (NO Bioavailability from Aortic Rings)

Brooks et al 


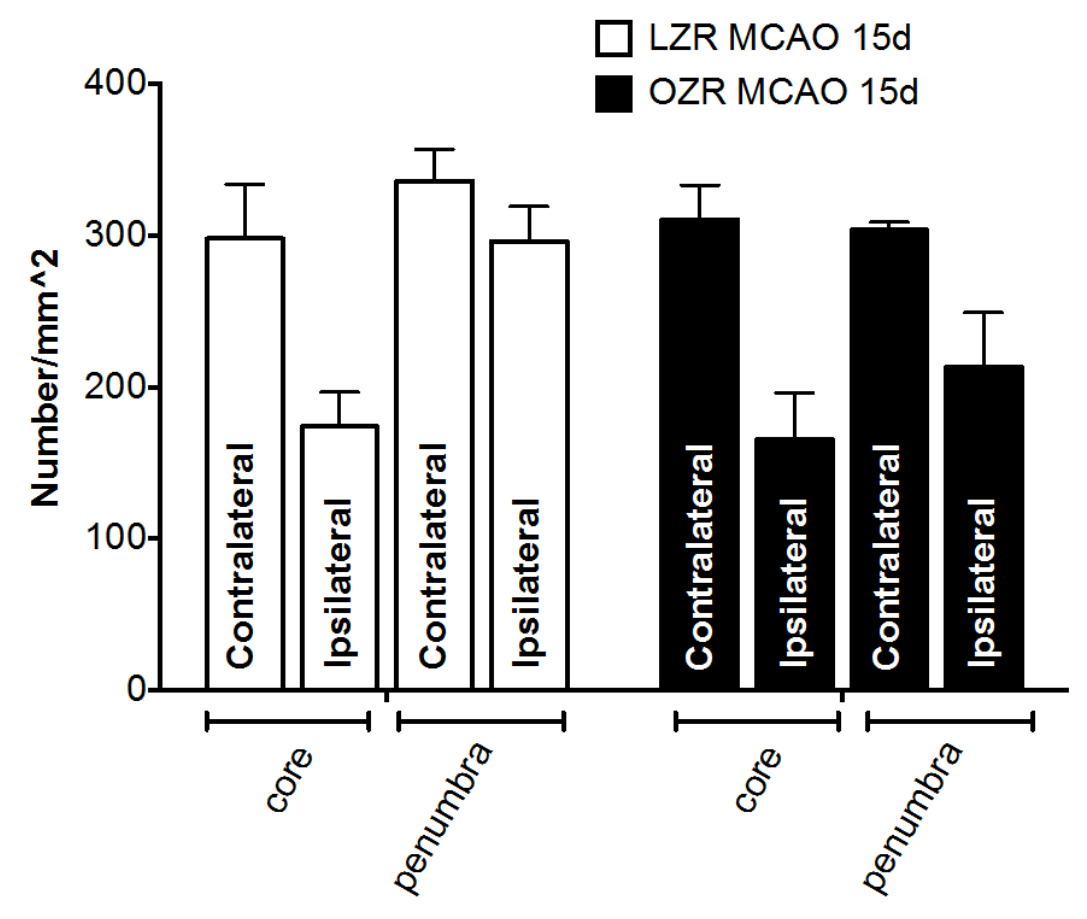

Figure 7 (Cerebral Microvessel Density) Brooks et al 


\section{Chapter 4: Conceptual Framework}

Chapter 4 established that ischemic stroke in rats with pre-existing MetSyn increased infarct size and mortality rates, impaired recovery of cerebral blood flow following reperfusion, and caused a progressive exacerbation of stroke-induced vascular dysfunction in both hemispheres of the brain. At two weeks post-stroke, reactivity in OZR IL MCA was completely eliminated, and vessels instead constricted to acetylcholine. Acute treatment with TEMPOL in OZR IL MCA prevented vasoconstriction, but did not improve dilation. These findings indicated that a specific injury, likely occurring during ischemia-reperfusion, was causing damage only to the OZR IL MCA. Administering the NAD(P)H oxidase 2 (Nox2) inhibitory peptide gp91ds-tat during ischemic stroke assessed whether oxidative stress generated during ischemia-reperfusion injury could be the cause. Treatment with gp91ds-tat was able to improve OZR IL MCA reactivity at 15 days post stroke to match OZR CL MCA, but had no effect on OZR CL MCA function. Nox 2 inhibition also improved post-stroke cerebral blood flow, and eliminated infarction in the stroke territory. The conclusion of this part of the study was that acute inhibition of Nox2, which is highly expressed on leukocytes that respond to ischemic injury, could prevent oxidative damage to both the IL MCA and the ischemic core of the stroke during and immediately following an ischemic event.

This chapter provided additional evidence that oxidative stress and ROS contribute to the loss of NO bioavailability that defines cerebrovascular dysfunction in MetSyn. The next chapter of this dissertation sought to establish whether chronic stress/depression, which is known to impair peripheral vascular reactivity, would further exacerbate these pathologies in OZR. Additionally, it investigates whether exercise training would prevent the onset of stress/depression-induced vasculopathy in both LZR and OZR. 
CHAPTER 5:

EXERCISE TRAINING PROTECTS AGAINST CHRONIC STRESS/DEPRESSIVE

BEHAVIOR INDUCED CERERBROVASCULAR DYSFUNCTION AND CORTICAL RAREFACTION IN LEAN AND OBESE ZUCKER RATS

Steven Brooks ${ }^{1,4}$, Shinichi Asano ${ }^{2,4}$, Kayla Branyan ${ }^{2,5}$, Evan DeVallance ${ }^{2,4}$, Roy Skinner ${ }^{2,4}$, Randall Bryner $^{2,4}$, I. Mark Olfert ${ }^{2,4}$, Paul D. Chantler ${ }^{2,3,4}$, and Jefferson C. Frisbee ${ }^{1,3,4}$

Department of Physiology and Pharmacology ${ }^{1}$, Division of Exercise Physiology ${ }^{2}$, Clinical and Translational Science Institute ${ }^{3}$, Center for Cardiovascular and Respiratory Sciences ${ }^{4}$; West Virginia University Health Sciences Center, Morgantown, WV

Send Correspondence to:

Jefferson C. Frisbee, Ph.D.

Department of Physiology and Pharmacology

Center for Cardiovascular and Respiratory Sciences

Robert C. Byrd Health Sciences Center

PO Box 9227

West Virginia University School of Medicine

Morgantown, WV 26505

Phone: (304) 293-6527

Fax: 304-293-5513

Email: jefrisbee@hsc.wvu.edu

Authorship Statement: SD Brooks contributed to this manuscript in terms of original idea, data collection, data analysis, primary synthesis of manuscript, and manuscript review. 


\section{ABSTRACT}

Objective: Chronic stress/depression is a major risk factor for cardiovascular disease (CVD) and impaired vascular function. The purpose of this study was twofold: first, to elucidate the functional and structural cerebrovascular pathologies induced by chronic stress/depression in a comorbid model of $\mathrm{CVD}$ /metabolic syndrome; second, to determine the efficacy of exercise training as a prophylactic method for abrogating the onset of stress-induced vasculopathy in LZR and OZR, and interrogate the mechanisms through which these benefits are conferred.

Methods: Eight weeks of an unpredictable chronic mild stress (UCMS) protocol was imposed on 9 week old male lean and obese Zucker rats (LZR, OZR) to induce depressive behaviors. Animals also underwent 8 weeks of daily treadmill exercise concurrent to the UCMS protocol. After sacrifice, brains were removed and the middle cerebral artery (MCA) was isolated and cannulated in an ex vivo microvessel preparation. Vascular reactivity was assessed to dilator and constrictor agonists, and again following acute incubation with TEMPOL or L-NAME. Changes in cerebral microvascular density were measured by fluorescence immunohistochemistry using a rat anti-CD31 antibody.

Results: Vascular reactivity was impaired in MCAs from both UCMS-LZR and OZR compared to non-stressed control. OZR-UCMS demonstrated near-total loss of reactivity to ACh, which was rescued marginally by TEMPOL. Exercise training was able to completely reverse the effects of UCMS in both LZR and OZR. Cerebral microvessel density did not vary significantly except in the cortex of UCMS OZR; this deficit was not present in UCMS Exercise OZR, however. Interestingly, both UCMS LZR and OZR showed a divergence in vascular benefit between good and poor responders to exercise. 
Conclusions: Chronic stress induces severe cerebral vasculopathy and rarefaction in rats with comorbid metabolic syndrome, but exercise training can protect the cerebrovasculature from the detrimental effects of chronic stress/depression. Our findings suggest NO bioavailability and levels of oxidative stress may be key factors in these pathologies. Patients suffering from chronic stress/depression and with other CVD comorbidities may experience severe vascular impairments and warrant further study. 


\section{INTRODUCTION}

Over 350 million people worldwide suffer from depression or a depressive disorder, establishing depression as a leading cause of disease burden [1, 2]. The National Institute of Mental Health (NIMH) reports that 15\% of U.S. adults suffer from depression [1-3], accounting for $\$ 150$ billion in direct costs and an estimated $\$ 193$ billion from lost earnings/lowered productivity. While the causes of depression are multifactorial, chronic psychological stress is recognized as a major factor contributing to the development of depressive episodes [4-10]. Glucocorticoid hormones, such as cortisol, become systemically elevated during periods of chronic stress and cause increased inflammatory signaling, impairments in metabolic processes [10-13], leading to dysregulation of the sympathetic-adrenalmedullary (SAM) and hypothalamicpituitary-adrenal (HPA) axes [8-10, 14-16]. Disruption of the normal homeostatic functions mediated by the HPA axis leads to insulin resistance, cortisol insensitivity, hyperphagia contributing to visceral fat, and systemic inflammation $[9,17-19]$.

Depression is an established independent risk factor for cardiovascular disease (CVD) [17, 20-23]. Patients suffering from depressive episodes, and no prior history of CVD, exhibit impaired flow-mediated dilation and endothelium-dependent dilation, as well as decreased plasma nitric oxide (NO) and endothelial NO synthase (eNOS) activity [4, 20, 24, 25]. Obesity, a major CVD risk factor, is also directly associated with depression [20]. Over a third of all US adults meet the criteria for the metabolic syndrome (MetSyn) [26, 27], and due to the nationwide prevalence of both MetSyn and depression-35\% and 15\% incidence respectively [26, 27]-many patients experience disease comorbidity. Dysregulation of the SAM and HPA axes have broad systemic effects that both directly and indirectly link the pathological progression of MetSyn and depression [14, 28-30], with implications for vascular function. The relationship between 
depression and CVD is compelling, indicating a critical need to evaluate the physiological interactions in a combined model.

The unpredictable chronic mild stress (UCMS) protocol is used to generate stressinduced depressive symptoms following exposure to unpredictable mild exogenous stressors [10, 11]. The UCMS model is considered translationally relevant, as long-term application induces symptoms similar to clinical depression [31-35]. Using the UCMS model, we [17, 32, 36] and others $[18,37,38]$, have demonstrated onset of symptoms, increases in systemic inflammation, and development of vascular dysfunction in large and small arteries. Degree of depressive symptom severity is significantly correlated with impairments to endothelium-dependent vasomotor responses in the peripheral and cerebral vasculatures $[17,32,36]$, characterized by increases in inflammatory markers and ROS, and a reduction in NO and PGI2 bioavailability.

The impact of chronic stress and depression on aortic ring reactivity is mediated through altered endothelial dysfunction, characterized by low nitric oxide [39] and prostacyclin bioavailability $[17,32,36]$. Similar mechanisms, including impaired endothelium-dependent reactivity and reduced NO bioavailability, as well as a pro-inflammatory $[6,18,40]$ and prothrombotic [41] circulatory microenvironment, has been observed in mice models of depression $[6,39,42]$, as well as in depressed patients $[24,25]$. These mechanisms of impaired vascular function themselves represent an independent predictor of risk for the development of CVD [25, 43].

We have also observed similar mechanisms of peripheral and cerebral vascular dysfunction in studies of the obese Zucker rat (OZR), an established model of MetSyn and its underlying network of systemic pathologies [44-46]. The OZR has a mutation that causes leptin insensitivity, leading to rapid onset of MetSyn from hyperphagia [44, 46]. We have previously 
reported that significant alterations in the regulation of blood flow in microvascular networks in the periphery $[45,47-55]$, and recently reported that middle cerebral arteries (MCA) in OZR develop significant alterations in myogenic tone [56], vascular reactivity [57], and vessel wall mechanics $[57,58]$ due to their underlying MetSyn. We also recently reported a progressive rarefaction of cerebral cortical microvessels in frontal cortex of OZR that was correlated with insulin resistance and nitric oxide (NO) bioavailability [58]. By imposing UCMS on OZR, this study aims to interrogate the functional and structural cerebrovascular impairments that develop due to comorbid depression and MetSyn.

Exercise training is an effective therapy for improving depressive symptoms and preventing recurrent episodes in patients suffering from depression [59]. Exercise training has also been shown to improve MetSyn symptoms on OZR, as well as increase microvascular perfusion and reduce vascular dysfunction [60,61]. Exercise has anti-oxidant benefits, and is known to increase NO bioavailability in both rats $[60,62]$ and humans $[59,63]$. The secondary aim of this study is to identify the prophylactic benefits of exercise training on cerebrovascular function in lean and obese Zucker rats undergoing the UCMS protocol, and elucidate the mechanisms through which protection is occurring.

\section{MATERIALS AND METHODS}

Animals: Male LZR and OZR (Harlan Laboratories) were fed standard chow and drinking water ad libitum and were housed in the animal care facility at the WVU HSC. All protocols received prior IACUC approval, and animals undergoing exercise training and/or chronic stress treatment were handled and inspected daily, with regular supervision of the veterinary support staff. At nine weeks of age, LZR and OZR were divided into four groups: age-matched control, UCMS 
(below), treadmill exercise, or UCMS with treadmill exercise. After eight weeks duration under either control, UCMS, exercise, or UCMS/Exercise conditions, rats were anesthetized with injections of sodium pentobarbital (50 mg・kg-1 i.p.) and a carotid artery was cannulated for determination of mean arterial pressure. Venous blood aliquots were collected by venipuncture for biochemical evaluation of plasma biomarkers of treatment outcomes and metabolic health status of the rats.

Unpredictable Chronic Mild Stress Protocol: All rats were singly housed; control animals were housed in a separate "quiet room" in close proximity to the room used for UCMS treatments. Exercise training was performed in an adjacent room to minimize animal transport. In UCMS groups, rats were randomly exposed to the following mild environmental stressors on multiple occasions throughout each 24 hour period over the course of 8 weeks:

1. Damp bedding $-10 \mathrm{oz}$. of water was added to each standard cage for the next 3 hours

2. Water - all bedding was removed and $\sim 0.5$ inches of water was added to empty cage for the next three hours. Water temperature was $\sim 30^{\circ} \mathrm{C}$ and the room temperature was $\sim 24^{\circ} \mathrm{C}$

3. Each cage was tilted to 45 degrees with or without bedding for 3 hours

4. Social stress - each rat was switched into a cage of a neighboring rat for 3 hours

5. No bedding lasting for 3 hours or, on two occasions each week, overnight.

6. Succession of light/dark cycles, lasting 30 minutes throughout a 24 hour period

7. Exposure to predator smells (e.g., cat fur) and/or sounds (e.g., cat growling) for 8 hours

8. After eight weeks, all rats were subjected to a series of behavioral tests to evaluate the outcomes of the UCMS procedures. 
Coat Status: This evaluation was done throughout the duration of the UCMS protocol. Each week, every rat was weighed and inspected for grooming habits. The total cumulative score was computed by giving an individual score of 0 (clean) or 1 (dirty) to eight body parts.

Exercise Training Protocol: LZR/OZR underwent 8 weeks of treadmill running, either concurrent with UCMS or as a standalone treatment. Animals ran 5 days/week on a three-lane motor driven treadmill set at a $4 \%$ grade. For the first 3 weeks, animals ran $20 \mathrm{~min} / \mathrm{day}$, increasing by $5 \mathrm{~min} /$ day until sustainable duration of 60 minutes daily was achieved. The following $15 \mathrm{~min}$. exercise intensity cycles were used: $24 \mathrm{~m} / \mathrm{min}$ for 10 minutes, $26 \mathrm{~m} / \mathrm{min}$ for 3 $\min$, and $28 \mathrm{~m} / \mathrm{min}$ for $2 \mathrm{~min}$. Mild electrical stimulation was used to encourage running; the maximum current was no more than .300 milliamps. Work calculations were performed based on animal body weight, and speeds/durations were adjusted to normalize work output between lean and obese animals accordingly.

Euthanasia and Collection of Middle Cerebral Arteries: While deeply anesthetized, each rat was decapitated, after which the brain was removed from the skull and placed in cold physiological salt solution (PSS; $4^{\circ} \mathrm{C}$ ). Both middle cerebral arteries (MCA) were dissected from their origin at the Circle of Willis and placed into an isolated microvessel chamber filled with PSS. Each MCA was subsequently doubly cannulated within in this heated chamber $\left(37^{\circ} \mathrm{C}\right)$ that allowed the lumen and exterior of the vessel to be perfused and superfused, respectively, with PSS from separate reservoirs. The PSS was equilibrated with a $21 \% \mathrm{O} 2,5 \% \mathrm{CO} 2$, and $74 \% \mathrm{~N} 2$ gas mixture and had the following composition (mM): $119 \mathrm{NaCl}, 4.7 \mathrm{KCl}, 1.17 \mathrm{MgSO} 4,1.6$ $\mathrm{CaCl} 2,1.18 \mathrm{NaH} 2 \mathrm{PO} 4,24 \mathrm{NaHCO} 3,0.026$ EDTA, and 5.5 glucose. Any side branches were ligated using a single strand teased from 6-0 suture. Vessel diameter was measured using television microscopy and an on-screen video micrometer. 
Measurements of Vascular Reactivity in Isolated MCA: Following cannulation, MCAs were extended to their in situ length and were equilibrated at $80 \%$ of the animal's mean arterial pressure to approximate in vivo perfusion pressure (Lombard paper). Any vessel that did not demonstrate significant active tone at the equilibration pressure was discarded. Active tone at the equilibration pressure was calculated as $(\Delta \mathrm{D} / \mathrm{Dmax}) \bullet 100$, where $\Delta \mathrm{D}$ is the diameter increase from rest in response to $\mathrm{Ca}^{2+-}$ free PSS, and Dmax is the maximum diameter measured at the equilibration pressure in $\mathrm{Ca}^{2+-}$ free PSS.

Following equilibration, the dilator reactivity of MCA was assessed in response to increasing concentrations of acetylcholine (ACh, $10^{-9} \mathrm{M}-10^{-5} \mathrm{M}$ ), sodium nitroprusside (SNP $10^{-}$ ${ }^{9} \mathrm{M}-10^{-5} \mathrm{M}$ ), and serotonin (5-hydroxytryptamine, 5-HT $10^{-10} \mathrm{M}-10^{-7} \mathrm{M}$ ). Vascular responses to acetylcholine (ACh) were also measured following acute incubation (45-60 minutes) with LNAME $\left(10^{-4} \mathrm{M}\right)$ and TEMPOL $\left(10^{-4} \mathrm{M}\right)$, to assess the contributions of nitric oxide (NO) and oxidative stress, respectively, in modulating vascular reactivity. (Cite Butcher JOVE). Passive vessel inner and outer diameters were measured at the end of experiments while vessel was immersed in $\mathrm{Ca}^{2+-}$ free PSS. Wall thickness was calculated as the difference in inner and outer passive vessel diameters.

Plasma Clinical Biomarker Analyses: Levels of common clinical plasma biomarkers (e.g. insulin, glucose, triglycerides, blood urea nitrogen (BUN)/creatinine, etc) were measured by the clinical laboratory service at Ruby Memorial Hospital (Morgantown, WV). Blood was drawn intravenously from anesthetized rats into lithium-heparin coated blood tubes and transported immediately to the laboratory for analysis.

Immunohistochemistry and Western blot: After rats were euthanized and surface vessels removed, brains were quickly saved in OCT and flash-frozen in super-cooled isopentane. 
Samples were stored at -80 until processing. Coronal brain slices (20 um thickness for histology) and additional brain slices (100um thickness for protein and RNA analysis) were prepared with a cryotome (Leica CM3050S; Leica Microsystems) from bregma -1 using a standard Rat Brain Atlas and transferred to charged microscope slides for histological analysis.

Cerebral microvessel and capillary density was evaluated by fluorescence immunohistochemistry using a rat anti-CD31 antibody (BD Biosciences, San Diego, CA) as described preciously (23632977). Briefly, brain sections were fixed with 4\% paraformaldehyde, permeabilized with $0.2 \%$ Triton X-100, and blocked with block solution (5\% BSA in PBS) and then incubated with anti-CD31 $(1: 100)$ for $1 \mathrm{~h}$ at room temperature in a humidified chamber. Following three washes with PBS, cells were incubated with Alexa Fluor 555-conjugated antimouse $\operatorname{IgG}(1: 1000$; Invitrogen) for $1 \mathrm{~h}$ at room temperature in a humidified chamber. After a final wash with PBS, cover glass was placed on mounting medium with DAPI (Vector Laboratories, Burlingame, CA) on a slide. Negative control was performed with the same procedure without primary antibody. Images were obtained with an epifluorescence microscope and a SPOT RT camera (Diagnostic Instruments). The microvessels density was analyzed in 6 region of interest ( 3 in cortex and 3 in striatum) in the MCA territory using ImageJ, as described previously (23632977).

Semi-quantitative immunodetection was performed as detailed previously. Briefly, brain tissues were homogenized in RIPA buffer (Thermo Fisher Scientific, Wiltham, MA) supplemented with $1 \mathrm{mM}$ DTT, protease and phosphatase inhibitor cocktails (Thermo Fisher Scientific, Wiltham, MA). Lysates were sonicated on ice and centrifuged at $10,000 \mathrm{xg}$ at $4{ }^{\circ} \mathrm{C}$ for $10 \mathrm{~min}$. The supernatant was removed and protein concentration was determined by BCA Protein assay (Thermo Fisher Scientific, Wiltham, MA). Samples were diluted to $1 \mathrm{mg}$ ml-1 in 
Laemmli sample buffer and boiled at $70{ }^{\circ} \mathrm{C}$ for $15 \mathrm{~min}$. Equivalent amounts of protein were separated by sodium dodecyl sulfate polyacrylamide gel electrophoresis (SDS-PAGE), followed by electroblotting onto nitrocellulose membranes. Membranes were blocked with 5\% milk in PBST for $1 \mathrm{~h}$ at room temperature, washed 3 times in phosphate buffered saline containing $0.1 \%$ Tween (PBST), and incubated overnight at $4{ }^{\circ} \mathrm{C}$ in primary antibodies against $\mathrm{CD} 31$ antibody (BD Biosciences, San Diego, CA), VEGF (1:250 SC-507), and $\square$ actin (1:2000 CST8457) Rabbit. After washing 3 times in TBST, membranes were incubated with anti-species secondary antibody conjugated with HRP and HRP-linked anti-biotin secondary antibodies for a biotinlyated ladder (1:5000; Cell Signaling Technology) for $1 \mathrm{~h}$ at room temperature and then washed again 3 times with TBST. An enhanced chemiluminescence detection system (ECL Advance, Amersham Biosciences) was used to detect the antibodies and the lanes were analyzed using a G:Box system (Syngene). The relative expression of each protein was quantified with ImageJ.

Data and Statistical Analyses: Mechanical responses following challenge with logarithmically increasing dosages of ACh, SNP, and 5-HT were fit with the three-parameter logistic equation:

$$
y=\min +\left[\frac{\max -\min }{1+10^{\log E D_{50}-x}}\right]
$$

Where $y$ represents the vessel diameter, "min" and "max" represent the lower (minimum) and upper (maximum) bounds, respectively, of the change in diameter with agonist concentration, $x$ is the logarithm of the agonist concentration, and $\operatorname{logED} 50$ represents the logarithm of the agonist concentration $(x)$ where the response $(y)$ is halfway between the bounds. For statistical evaluation of results, we have focused on the changes in the upper bounds as a representation of vessel reactivity, as lower bound remained consistent between all groups (defined as the pre- 
challenge diameter); we did not determine a consistent or significant change to the logED50 values between treatment groups. All other statistical analyses utilized Student's t-test or analysis of variance (ANOVA) with Student-Newman-Keuls test post-hoc. Statistical significance is represented by $\mathrm{p}<0.05$.

\section{RESULTS}

Clinical biomarkers from all animal groups were measured from plasma. As expected, OZR control demonstrated elevated plasma glucose, insulin, triglycerides, and cholesterol (especially LDL), as well as elevated alkaline phosphatase, blood urea nitrogen (BUN) and BUN/creatinine ratio, AST, and ALT, when compared to LZR control. LZR UCMS developed higher levels of plasma glucose, triglycerides, and AST, ALT compared to LZR control; however, these biomarker levels were brought back to control levels in the exercise treated LZR UCMS cohort. In OZR UCMS, plasma glucose, insulin, triglycerides, and cholesterol, as well as BUN, BUN creatinine ration, alkaline phosphatase, ALT, and AST, all remained significantly elevated compared to LZR control, but were not different from OZR control. Exercise treated OZR UCMS had reduced cholesterol (including LDL) and improved insulin levels compared to OZR control and OZR UCMS, while the other biomarkers remained unchanged. In exercise treated non-stressed OZR, insulin was also lowered, as were cholesterol, LDL cholesterol, and alkaline phosphatase, while glucose, BUN/creatinine, triglycerides, and AST, ALT remained unchanged. (plasma results not shown due to unavailability of graph).

Vascular reactivity was measured first in MCA from UCMS and control LZR and OZR to assess the effect of chronic stress on cerebrovascular reactivity. Figure 1 presents endothelium-dependent vasodilation of ex vivo MCA to escalating doses of ACh. LZR control 
dilated most robustly at all doses compared to LZR UCMS, OZR control, and OZR UCMS (Figure 1A). Maximum dilation (response to ACh $\left[10^{-5}\right] \mathrm{M}$ ) was reduced by $\sim 30 \%$ in LZR UCMS compared to LZR control, $\sim 45 \%$ in OZR control, and more than $65 \%$ in OZR UCMS (Figure 1B). MCA incubation was TEMPOL was able to almost completely rescue vasodilation to ACh in LZR UCMS, while incubation with L-NAME eliminated $\sim 80 \%$ of vasodilation (Figure 1C). In OZR UCMS, TEMPOL incubation restored MCA dilation to that of OZR control, while L-NAME incubation completely eliminated all reactivity to ACh in OZR UCMS MCA (Figure 1D).

Figure 2 presents maximum dilation to the highest dose of ACh $\left[10^{-5}\right] \mathrm{M}$ or SNP $\left[10^{-5}\right] \mathrm{M}$, assessing endothelium-dependent and endothelium-independent vasomotor responses respectively in control and UCMS treated LZR and OZR. In LZR control, incubation with TEMPOL did not improve vasodilation to ACh, and dilation to SNP was not significantly different from ACh (Figure 2A). Incubation with L-NAME significantly reduced maximum vasodilation to ACh. In LZR UCMS, max dilation to ACh was reduced $~ 30 \%$ compared to LZR control, while dilation to SNP was not different from LZR control, indicating the difference in vasoreactivity is largely attributable to endothelium-dependent mechanisms (Figure 2B). Incubation with TEMPOL significantly improved maximum dilation to ACh, while L-NAME reduced dilation consistent with its effect in LZR control MCA (Figure 2B). In OZR control, maximum dilation to ACh was reduced $\sim 45 \%$ compared to LZR control, and dilation to SNP was reduced as well, by $\sim 15 \%$ (Figure $2 \mathrm{C}$ ). Treatment with TEMPOL improved dilation to ACh by nearly $50 \%$, increasing max dilation to $75 \%$ of LZR control; treatment with L-NAME nearly ablated all vasodilation to ACh (Figure 2C). In OZR UCMS, max dilation to ACh was decreased $\sim 70 \%$ or $\sim 45 \%$ to LZR control/OZR control respectively, and dilation to SNP was reduced a 
further $\sim 10 \%$ compared to OZR control (Figure 2D). Incubation with TEMPOL improved dilator response to ACh by 75\%, restoring lost reactivity between OZR and OZR UCMS, while incubation with L-NAME totally elimination vasodilation to $\mathrm{ACh}$, instead allowing vasoconstriction in some vessels (Figure 2D).

Exercise training was then imposed on control and UCMS LZR and OZR over 8 weeks to assess the effects of exercise on behavioral and vascular outcomes of UCMS, MetSyn, and comorbid UCMS/MetSyn. Figure 3 presents the effects of exercise training on MCA vasoreactivity in LZR control, LZR UCMS, OZR control, and OZR UCMS. Dilation to escalating doses of ACh was not different between LZR control and LZR exercise, and dilation in LZR UCMS Exercise was restored to LZR control levels (Figure 3A). In contrast, dilation to ACh in OZR exercise was significantly improved compared to OZR control, recovering nearly 90\% of dilation compared to LZR control (Figure 3B). Exercise training in OZR UCMS also reversed the decline in dilation seen in OZR UCMS, improving dilation over OZR control by a further $\sim 15 \%$ (Figure 3B). Maximum dilation to SNP $\left[10^{-5}\right] \mathrm{M}$ did not significantly differ between any of the LZR treatment groups (Figure 3C), while dilation to SNP $\left[10^{-5}\right] \mathrm{M}$ did improve significantly in OZR exercise, returning it to levels comparable to LZR control (Figure 3D). Exercise training did not improve maximum dilation to SNP in OZR UCMS, however.

To account for whether structural changes in the vessels may account for differences in maximal dilation, MCAs were allowed to equilibrate in a $\mathrm{Ca} 2+$ free PSS solution resulting in passive dilation. These results are presented in Figure 4. Passive inner MCA diameter did not change for LZR Exercise or LZR UCMS Exercise compared to LZR control; interestingly, a significant increase in inner diameter was found in LZR UCMS (Figure 4A). However, this was also reflected as a comparable significant increase in passive MCA outer diameter, indicating 
that this cohort of animals may have just genetically had slightly larger MCAs (Data not shown). Passive inner diameters of OZR and OZR UCMS were both significantly reduced compared to LZR control, while inner diameters of OZR Exercise and OZR UCMS Exercise were not significantly different from LZR control (Figure 4B). The measured wall thickness for all LZR groups (Figure 4C) and all OZR groups (Figure 4D) was not significantly different, meaning that significant differences in passive inner MCA diameter were attributable to eutrophic inward (or outward) remodeling, as opposed to hypertrophic remodeling.

To further interrogate the mechanisms governing endothelium-dependent vasodilation in exercise treated animals, isolated MCA were incubated with either TEMPOL or L-NAME. Maximum dilation to $\mathrm{ACh}$ in exercise-trained OZR is presented in Figure 5. As stated previously, MCAs from OZR Exercise showed a significantly increased maximum dilation to ACh $\left[10^{-5}\right] \mathrm{M}$ compared to OZR control. Incubation with TEMPOL further increased dilation, recovering it to levels equivalent of LZR control. Incubation with L-NAME reduced dilation by $\sim 60 \%$, which while significant, was less that the reduction measured in LZR control to the same dose of L-NAME. Figure 6 presents maximum dilation in exercise trained LZR UCMS and OZR UCMS. Incubation with TEMPOL did not have a significant effect on dilation to ACh in LZR UCMS Exercise, while the effect of L-NAME was the same as LZR Control (Figure 6A). In OZR UCMS Exercise, TEMPOL also did not improve dilation to ACh, in contrast to OZR Exercise and OZR UCMS (Figure 6B). Similar to OZR UCMS, L-NAME completely ablated all dilation to $\mathrm{ACh}$, and allowed vasoconstriction in some MCAs.

Analysis of vasodilator responses in MCA of OZR Exercise, OZR UCMS Exercise, and LZR UCMS Exercise groups revealed larger Standard Errors than any other treatment condition. Subsequent analysis revealed that some OZR and UCMS MCAs were 'poor' responders while 
others were 'good' responders to $\mathrm{ACh}$; evaluation of the training log revealed that these animals were also 'good' or 'poor' performers on the treadmill, often requiring different levels of encouragement to run. Analysis of each of these three groups was therefore further sub-divided into 'good' responders $(n=4$ OZR Exercise, $n=4$ OZR UCMS Exercise, $n=4$ LZR UCMS Exercise) and 'poor' responders ( $\mathrm{n}=3$ OZR Exercise, $n=3$ OZR UCMS Exercise, $n=4$ LZR UCMS Exercise), which were presented in Figure 7. For OZR exercise, 'good' responders showed maximum dilation responses to ACh on par with LZR control, while 'poor' responders showed a much reduced, but still significant, benefit from exercise (Figure 7A). In OZR UCMS Exercise, 'good' responders showed a significant improvement in vasodilation, while 'poor' responders did not significantly improve compared to OZR UCMS (Figure 7B). In LZR UCMS Exercise, this trend continued, as 'good' responders demonstrated improved dilation to levels comparable to LZR control, while 'poor' responders were not significantly different from LZR UCMS (Figure 7C). Importantly, animals in the LZR exercise group did not develop divergent dilatory responses, and training logs indicated universally 'good' performance during training.

Vasoconstriction of MCAs from control, UCMS, and UCMS Exercise LZR and OZR are presented in Figure 8. Vasoconstriction to 5-HT was similar between LZR control and LZR exercise. LZR UCMS constricted significantly more at the higher doses of 5-HT than LZR control, which was recovered back to control responses in LZR UCMS Exercise (Figure 8A). OZR control and OZR UCMS both showed significant, and comparable, increases in constriction compared to LZR control, but exercise training was not able to significantly improve constriction in OZR UCMS as it was in LZR UCMS (Figure 8B). Exercise was, however, able to restore constriction in OZR to levels comparable to LZR control. Maximum constrictor responses to 5- 
HT $\left[10^{-7}\right] \mathrm{M}$ are presented for LZR (Figure 8C) and OZR (Figure 8D) to allow visualization of responses between LZR and OZR.

Figure 9 presents the results of fluorescence immunohistochemistry staining for anti-CD31 to measure microvascular density in the cortex and striatum of MCA perfusion territories, and semi-quantitative immunodetection by western blot of these brain regions for CD-31 and VEGF. CD-31+ vessel density in the cortex was not significantly different between LZR control, LZR UCMS, and LZR UCMS Exercise, nor between LZR Control, OZR Control, and OZR Exercise (Figure 9A). However, there is a sharp decline in microvessel density in OZR UCMS, which is recovered fully in OZR UCMS Exercise (Figure 9A). No differences in microvessel density in the striatum achieved significance between any groups, but OZR UCMS trended in that direction (Figure 9B). Protein levels of CD31 were also significantly decreased in OZR UCMS (Figure 9C), but no other groups, while protein levels of VEGF trended towards increases in both LZR UCMS Exercise and OZR UCMS Exercise compared to LZR UCMS and OZR UCMS respectively (Figure 9D).

\section{DISCUSSION}

We present here novel findings that show cerebrovascular dysfunction in the MCAs of LZR and OZR following eight weeks of UCMS. To our knowledge, this is the first such report of stress/depression-induced cerebrovascular dysfunction in rats. The comorbid presence of chronic stress and MetSyn resulted in a severe reduction to endothelium-dependent vasodilation of MCAs, as well as impaired dilation to an exogenous donor of NO, significant eutrophic inward remodeling, and a decrease in cortical microvessel density in the MCA perfusion territory. We also report that exercise training, which recovers vascular function in OZR controls to levels 
comparable with LZR, is able to completely reverse the onset of vascular pathologies from UCMS in LZR, and block vascular impairment present in control OZR from progressing in the presence of UCMS.

These impairments, and subsequent improvements following exercise training, are likely a function of limited NO bioavailability. This is supported through our observation of a decrease in direct production of NO from conduit vessels, and improved MCA reactivity following incubation with TEMPOL/ total loss of reactivity following incubation with L-NAME. Nitric oxide has been extensively described in the literature as being the most influential vasodilatory mediator produced in the cerebrovascular endothelium [64-71], and impairments in NO bioavailability have been linked with cerebrovascular dysfunction in diabetes, metabolic syndrome, hypertension, and stroke [72-76]. Reduced NO bioavailability has also been linked with rarefaction in peripheral tissues [49], as well as with progressive increase in vascular dysfunction of skeletal muscle arterioles $[50,51,77]$. Oxidant stress, which is exacerbated by chronic stress, is well established as a leading cause of vascular dysfunction, and these results support that it may indeed drive loss of NO bioavailability and the progression of cerebral vasculopathy reporter here.

Chronic stress, which causes dysregulation of the SAM and HPA axes, has been linked with a litany of metabolic $[21,39,78]$ and vascular consequences. We previously reported loss of NO bioavailability and increases in biomarkers of oxidant stress in a murine model of UCMS $[17,36]$, which correlated with impaired endothelium-dependent mechanisms of vasodilation. Our results in the present study confirm similar mechanisms of dysfunction in the cerebrovasculature of LZR. Baseline impairments in reactivity of MCA from OZR are also consistent with our previously published work, with further impairment resulting from the 
imposition of UCMS in OZR. Of concern, we did not see a decrease in microvessel density in control OZR, as we have previously reported [58]; this may be a result of studying a different region of interest in the brain- namely, the MCA perfusion territory (this study) as opposed to the frontal cortex. The MCA perfusion territory is the largest and most well-perfused area of the cortex, and is well-supplied with collateral flow. If the basal level of collateral flow is sufficient to prevent rarefaction in this region even in OZR, then the sharp decline in microvessel density seen in OZR UCMS is of great concern, as this region is the most commonly implicated in ischemic stroke, and impaired perfusion/reduction of collaterals would worsen stroke outcomes.

The eutrophic inward remodeling reported here is also consistent with advanced vascular dysfunction seen in OZR [57]. We previously reported that chronic anti-oxidant therapy was able to prevent such structural alterations, lending further support for the argument that loss of NO bioavailability and increased oxidant stress are driving the pathological changes in the cerebrovascular circulation of OZR. Vessel diameter, functional hyperemic responses, and vascular density all play a role in maintaining adequate blood flow to each region of the brain, and must be able to response accordingly to accommodate increases in flow during periods of higher neural activity. Impairments in any/all of these processes may have significant impacts on neuronal metabolism and activity.

Exercise training has been extensively researched as a treatment for depression and major depressive disorders [59]. Our results show that exercise training was able to improve cerebrovascular outcomes in terms of vascular reactivity, remodeling, and microvessel density, in both OZR and LZR following 8 weeks of UCMS. In humans, exercise has been found to be as effective as SSRIs at improving depressive symptoms [59] and more effective than several common drugs at preventing recurrent episodes of depression. Large meta-analyses have 
determined that exercise programs of any kind- aerobic, weight training, walking, etc- were all equally effective as treatments as long as it was 9 weeks or longer [59]. Clinical studies have also shown that exercise is equally effective for men and women, as well as newly diagnosed or longterm diagnosed patients. The mechanisms through which exercise treats depression are currently an area of debate. One theory is that improved bioavailability of mono-amine neurotransmitters, including NE, dopamine, and serotonin, can lead to improved neurological function in the affected areas of the brain [40, 79-81]. Alternatively, a reduction in oxidant stress, and increase in overall body metabolism have been identified as ways exercise combats depression [59, 62, $68,82]$.

Recent studies into the etiology of depression have linked molecular alterations in hippocampal neurons with increases in oxidant stress [6, 83-85]. Two recent studies have shown that culturing hippocampal neurons from depressed animals with TEMPOL can restore rates of proliferation, and direct anti-oxidant treatment of the hippocampus in mice has been shown to be anti-depressant. As reactive oxygen species (ROS) can cross the blood brain barrier, peripheral sources of ROS may therefore contribute to the oxidative microenvironment driving loss of neuronal proliferation and impaired neuronal function in the hippocampus. By this theory, exercise training may be anti-depressant by its anti-oxidative effects, in addition to its effects on mono-amine neurotransmitter production. This would provide evidence to the theory that systemic oxidative stress and inflammation, both common consequences of MetSyn and most types of CVD, contributes directly to the development of depression. Development of physiological neuronal dysfunction may therefore share a common pathological etiology with the vascular dysfunction, as both develop due to systemic oxidative stress. Furthermore, impairment in delivery of blood to the hippocampus could cause hypoxia, inducing oxidative dysregulation 
within neurons and contributing to the pathology. Further work is certainly warranted to study this link between CVD, vascular dysfunction, and the molecular origins of depression in the hippocampus.

\section{Clinical Implications}

Impaired regulation of cerebral blood flow has significant implications for cognitive function, as well as for risk and outcomes in disease such as mild cognitive impairment, vascular dementia, and ischemic stroke. Mild cognitive impairments and vascular dementia have been previously linked to poor regulation of cerebral perfusion. The cerebrovascular adaptations occurring from MetSyn and UCMS may therefore represent key contributors to multiple cerebral pathologies [70, 71, 86, 87]. We have previously reported a decrease in cerebral cortical microvascular density associated with MetS, and report here that this rarefaction may increase in the presence of chronic stress. In this study, we report that limited NO bioavailability and oxidant stress, stemming from both MetSyn and UCMS, contribute to impaired cerebral perfusion by reducing endothelial-dependent dilation, inward remodeling of MCA, and reducing density of microvessels. These mechanisms serve as physiological contributors to impairments in cerebral blood flow regulation and perfusion, which have been linked to cognitive decline with aging. Indeed, recent work by Tucsek et al [88-91] found that combining aging with high-fat diet induced obesity in mice led to an exacerbation of cerebrovascular rarefaction and neurovascular

uncoupling which was directly associated with cognitive impairments. MetSyn- associated cerebrovascular disease leads to elevated risk for ischemic stroke risk for patients with MetSyn.

Early recognition of stress/depression-associated vascular impairments, especially in patients who concurrently suffer from MetSyn, may provide a valuable clinical time window to treat patients to protect their cerebral vasculature. Prophylactically treating such patients with 
drugs that confer pleiotropic benefits- such as atorvastatin $[52,57,92]$ - may limit the deleterious impact of chronic stress on neurological and cerebrovascular health in patients with MetSyn, potentially conferring the anti-depressant and vasculo-protective benefits of exercise in a patient popular who may be unwilling or unable to exercise daily.

\section{Acknowledgements:}

The authors would like to thank WVU Department of Exercise Physiology and Dr. Randall Bryner for arranging extensive undergraduate student support for the stress protocol and exercise training protocol used in this paper. The authors would also like to thank the WVU Animal Care facilities for excellent technical support and animal monitoring during these procedures, as well as Kent Lemaster, Ashlee Seldomridge, and Whitney Sheets for technical support. Additionally, we also acknowledge the support provided through Center for Cardiovascular and Respiratory Sciences and the Clinical and Translational Sciences Institute at the West Virginia University Health Sciences Center.

\section{Grants}

This study was supported by the American Heart Association grants IRG 14330015, PRE 16850005, EIA 0740129N and pre-doctoral fellowship AHA 14PRE 20380386. Research reported in this publication was supported by the National Institute of General Medical Sciences of the National Institutes of Health under Award Number U54GM104942.

\section{Disclaimer}

The content is solely the responsibility of the authors and does not necessarily represent the official views of the National Institutes of Health. 


\section{LITERATURE CITED:}

1. CDC.gov, Current Depression Among Adults --- United States, 2006 and 2008. MMWR 2010. 59(38): p. 1229-1235.

2. NIMH. Depression. 2013 [cited 2103.

3. Kessler, R.C., et al., Sex and depression in the National Comorbidity Survey. I: Lifetime prevalence, chronicity and recurrence. J Affect Disord, 1993. 29(2-3): p. 85-96.

4. Chen, H., et al., Relationship of depression, stress and endothelial function in stable angina patients. Physiol Behav, 2013. 118: p. 152-8.

5. Lett, H.S., et al., Depression as a risk factor for coronary artery disease: evidence, mechanisms, and treatment. Psychosom Med, 2004. 66(3): p. 305-15.

6. Lucca, G., et al., Effects of chronic mild stress on the oxidative parameters in the rat brain. Neurochem Int, 2009. 54(5-6): p. 358-62.

7. Young, E.A., et al., Alteration in the hypothalamic-pituitary-ovarian axis in depressed women. Arch Gen Psychiatry, 2000. 57(12): p. 1157-62.

8. Barden, N., Implication of the hypothalamic-pituitary-adrenal axis in the physiopathology of depression. J Psychiatry Neurosci, 2004. 29(3): p. 185-93.

9. de Kloet, C.S., et al., Assessment of HPA-axis function in posttraumatic stress disorder: pharmacological and non-pharmacological challenge tests, a review. J Psychiatr Res, 2006. 40(6): p. 550-67.

10. Pariante, C.M. and A.H. Miller, Glucocorticoid receptors in major depression: relevance to pathophysiology and treatment. Biol Psychiatry, 2001. 49(5): p. 391-404.

11. Burke, H.M., et al., Depression and cortisol responses to psychological stress: a metaanalysis. Psychoneuroendocrinology, 2005. 30(9): p. 846-56.

12. DeRijk, R. and E.R. de Kloet, Corticosteroid receptor genetic polymorphisms and stress responsivity. Endocrine, 2005. 28(3): p. 263-70.

13. Raison, C.L. and A.H. Miller, When not enough is too much: the role of insufficient glucocorticoid signaling in the pathophysiology of stress-related disorders. Am J Psychiatry, 2003. 160(9): p. 1554-65.

14. Antonijevic, I.A., Depressive disorders -- is it time to endorse different pathophysiologies? Psychoneuroendocrinology, 2006. 31(1): p. 1-15. 
15. Parker, K.J., A.F. Schatzberg, and D.M. Lyons, Neuroendocrine aspects of hypercortisolism in major depression. Horm Behav, 2003. 43(1): p. 60-6.

16. Young, E.A., S.C. Ribeiro, and W. Ye, Sex differences in ACTH pulsatility following metyrapone blockade in patients with major depression. Psychoneuroendocrinology, 2007. 32(5): p. 503-7.

17. d'Audiffret, A.C., et al., Depressive behavior and vascular dysfunction: a link between clinical depression and vascular disease? J Appl Physiol (1985), 2010. 108(5): p. 1041-51.

18. Munhoz, C.D., et al., Stress-induced neuroinflammation: mechanisms and new pharmacological targets. Braz J Med Biol Res, 2008. 41(12): p. 1037-46.

19. Rohleder, N., Acute and chronic stress induced changes in sensitivity of peripheral inflammatory pathways to the signals of multiple stress systems --2011 Curt Richter Award Winner. Psychoneuroendocrinology, 2012. 37(3): p. 307-16.

20. Bornstein, S.R., et al., Approaching the shared biology of obesity and depression: the stress axis as the locus of gene-environment interactions. Mol Psychiatry, 2006. 11(10): p. 892-902.

21. Ippoliti, F., N. Canitano, and R. Businaro, Stress and obesity as risk factors in cardiovascular diseases: a neuroimmune perspective. J Neuroimmune Pharmacol, 2013. 8(1): p. 212-26.

22. Maddock, C. and C.M. Pariante, How does stress affect you? An overview of stress, immunity, depression and disease. Epidemiol Psichiatr Soc, 2001. 10(3): p. 153-62.

23. Plante, G.E., Depression and cardiovascular disease: a reciprocal relationship. Metabolism, 2005. 54(5 Suppl 1): p. 45-8.

24. Chrapko, W.E., et al., Decreased platelet nitric oxide synthase activity and plasma nitric oxide metabolites in major depressive disorder. Biol Psychiatry, 2004. 56(2): p. 129-34.

25. Dimopoulos, N., et al., Elevation of plasma concentration of adhesion molecules in late-life depression. Int J Geriatr Psychiatry, 2006. 21(10): p. 965-71.

26. Aguilar, M., et al., Prevalence of the metabolic syndrome in the United States, 2003-2012. JAMA, 2015. 313(19): p. 1973-4.

27. American Heart Association. Metabolic Syndrome. 2014 [cited 20163 March]; Available from: http://www.heart.org/HEARTORG/Conditions/More/MetabolicSyndrome/MetabolicSyndrome_UCM_002080_SubHomePage.jsp. 
28. Francis, G.S., Interaction of the sympathetic nervous system and electrolytes in congestive heart failure. Am J Cardiol, 1990. 65(10): p. 24E-27E; discussion 52E.

29. Francis, G.S., Neurohormonal control of heart failure. Cleve Clin J Med, 2011. 78 Suppl 1: p. S75-9.

30. Francis, G.S. and J.N. Cohn, The autonomic nervous system in congestive heart failure. Annu Rev Med, 1986. 37: p. 235-47.

31. Grippo, A.J., et al., Chronic mild stress induces behavioral and physiological changes, and may alter serotonin $1 A$ receptor function, in male and cycling female rats. Psychopharmacology (Berl), 2005. 179(4): p. 769-80.

32. Isingrini, E., et al., Altered aortic vascular reactivity in the unpredictable chronic mild stress model of depression in mice: UCMS causes relaxation impairment to ACh. Physiol Behav, 2011. 103(5): p. 540-6.

33. Mineur, Y.S., C. Belzung, and W.E. Crusio, Effects of unpredictable chronic mild stress on anxiety and depression-like behavior in mice. Behav Brain Res, 2006. 175(1): p. 43-50.

34. Porsolt, R.D., Animal models of depression: utility for transgenic research. Rev Neurosci, 2000. 11(1): p. 53-8.

35. Willner, P., Validity, reliability and utility of the chronic mild stress model of depression: a 10-year review and evaluation. Psychopharmacology (Berl), 1997. 134(4): p. 319-29.

36. Stanley, S.C., et al., Protective effect of sex on chronic stress- and depressive behaviorinduced vascular dysfunction in BALB/cJ mice. J Appl Physiol (1985), 2014. 117(9): p. 959-70.

37. Bayramgurler, D., et al., The effect of etanercept on aortic nitric oxide-dependent vasorelaxation in an unpredictable chronic, mild stress model of depression in rats. Eur J Pharmacol, 2013. 710(1-3): p. 67-72.

38. Kamper, E.F., et al., Sex differences in oxidant/antioxidant balance under a chronic mild stress regime. Physiol Behav, 2009. 98(1-2): p. 215-22.

39. Peng, Y.L., et al., Inducible nitric oxide synthase is involved in the modulation of depressive behaviors induced by unpredictable chronic mild stress. J Neuroinflammation, 2012. 9: p. 75.

40. Haroon, E., C.L. Raison, and A.H. Miller, Psychoneuroimmunology meets neuropsychopharmacology: translational implications of the impact of inflammation on behavior. Neuropsychopharmacology, 2012. 37(1): p. 137-62. 
41. Pinto, V.L., et al., Low plasma levels of L-arginine, impaired intraplatelet nitric oxide and platelet hyperaggregability: implications for cardiovascular disease in depressive patients. $\mathrm{J}$ Affect Disord, 2012. 140(2): p. 187-92.

42. Isingrini, E., et al., Fluoxetine effect on aortic nitric oxide-dependent vasorelaxation in the unpredictable chronic mild stress model of depression in mice. Psychosom Med, 2012. 74(1): p. 63-72.

43. Rajagopalan, S., et al., Abnormal brachial artery flow-mediated vasodilation in young adults with major depression. Am J Cardiol, 2001. 88(2): p. 196-8, A7.

44. Aleixandre de Artinano, A. and M. Miguel Castro, Experimental rat models to study the metabolic syndrome. Br J Nutr, 2009. 102(9): p. 1246-53.

45. Frisbee, J.C. and M.D. Delp, Vascular function in the metabolic syndrome and the effects on skeletal muscle perfusion: lessons from the obese Zucker rat. Essays Biochem, 2006. 42: p. 14561.

46. Tofovic, S.P. and E.K. Jackson, Rat models of the metabolic syndrome. Methods Mol Med, 2003. 86: p. 29-46.

47. Butcher, J.T., A.G. Goodwill, and J.C. Frisbee, The ex vivo isolated skeletal microvessel preparation for investigation of vascular reactivity. J Vis Exp, 2012(62).

48. Chantler, P.D. and J.C. Frisbee, Arterial function in cardio-metabolic diseases: from the microcirculation to the large conduits. Prog Cardiovasc Dis, 2015. 57(5): p. 489-96.

49. Frisbee, J.C., Reduced nitric oxide bioavailability contributes to skeletal muscle microvessel rarefaction in the metabolic syndrome. Am J Physiol Regul Integr Comp Physiol, 2005. 289(2): p. R307-R316.

50. Frisbee, J.C., et al., Integration of skeletal muscle resistance arteriolar reactivity for perfusion responses in the metabolic syndrome. Am J Physiol Regul Integr Comp Physiol, 2009. 296(6): p. R1771-82.

51. Frisbee, J.C., et al., Altered mechanisms underlying hypoxic dilation of skeletal muscle resistance arteries of hypertensive versus normotensive Dahl rats. Microcirculation, 2001. 8(2): p. 115-27.

52. Goodwill, A.G., et al., Impact of chronic anticholesterol therapy on development of microvascular rarefaction in the metabolic syndrome. Microcirculation, 2009. 16(8): p. 667-84. 
53. Goodwill, A.G., M.E. James, and J.C. Frisbee, Increased vascular thromboxane generation impairs dilation of skeletal muscle arterioles of obese Zucker rats with reduced oxygen tension. Am J Physiol Heart Circ Physiol, 2008. 295(4): p. H1522-8.

54. Phillips, S.A., F.A. Sylvester, and J.C. Frisbee, Oxidant stress and constrictor reactivity impair cerebral artery dilation in obese Zucker rats. Am J Physiol Regul Integr Comp Physiol, 2005. 288(2): p. R522-30.

55. Stapleton, P.A., et al., Altered mechanisms of endothelium-dependent dilation in skeletal muscle arterioles with genetic hypercholesterolemia. Am J Physiol Regul Integr Comp Physiol, 2007. 293(3): p. R1110-9.

56. Butcher, J.T., et al., Differential impact of dilator stimuli on increased myogenic activation of cerebral and skeletal muscle resistance arterioles in obese zucker rats. Microcirculation, 2013. 20(7): p. 579-89.

57. Brooks, S.D., et al., Metabolic syndrome impairs reactivity and wall mechanics of cerebral resistance arteries in obese Zucker rats. Am J Physiol Heart Circ Physiol, 2015. 309(11): p. H1846-59.

58. Chantler, P.D., et al., Cerebral Cortical Microvascular Rarefaction in Metabolic Syndrome is Dependent on Insulin Resistance and Loss of Nitric Oxide Bioavailability. Microcirculation, 2015. 22(6): p. 435-45.

59. Craft, L.L. and F.M. Perna, The Benefits of Exercise for the Clinically Depressed. Prim Care Companion J Clin Psychiatry, 2004. 6(3): p. 104-111.

60. Frisbee, J.C., et al., Exercise training blunts microvascular rarefaction in the metabolic syndrome. Am J Physiol Heart Circ Physiol, 2006. 291(5): p. H2483-92.

61. Peterson, J.M., et al., Mitochondrial apoptotic signaling is elevated in cardiac but not skeletal muscle in the obese Zucker rat and is reduced with aerobic exercise. J Appl Physiol (1985), 2008. 105(6): p. 1934-43.

62. Graham, D.A. and J.W. Rush, Exercise training improves aortic endothelium-dependent vasorelaxation and determinants of nitric oxide bioavailability in spontaneously hypertensive rats. J Appl Physiol (1985), 2004. 96(6): p. 2088-96.

63. Rush, J.W., S.G. Denniss, and D.A. Graham, Vascular nitric oxide and oxidative stress: determinants of endothelial adaptations to cardiovascular disease and to physical activity. Can $\mathrm{J}$ Appl Physiol, 2005. 30(4): p. 442-74. 
64. Chan, S.L., et al., Pial Collateral Reactivity During Hypertension and Aging: Understanding the Function of Collaterals for Stroke Therapy. Stroke, 2016. 47(6): p. 1618-25.

65. Doughan, A.K., D.G. Harrison, and S.I. Dikalov, Molecular mechanisms of angiotensin IImediated mitochondrial dysfunction: linking mitochondrial oxidative damage and vascular endothelial dysfunction. Circ Res, 2008. 102(4): p. 488-96.

66. Faraci, F.M., C. Lynch, and K.G. Lamping, Responses of cerebral arterioles to ADP: eNOSdependent and eNOS-independent mechanisms. Am J Physiol Heart Circ Physiol, 2004. 287(6): p. H2871-6.

67. Fouyas, I.P., et al., Cerebrovascular responsiveness to NG-nitro-L-arginine methyl ester in spontaneously diabetic rats. Br J Pharmacol, 1996. 118(2): p. 243-8.

68. Mayhan, W.G., et al., Exercise training normalizes impaired NOS-dependent responses of cerebral arterioles in type 1 diabetic rats. Am J Physiol Heart Circ Physiol, 2011. 300(3): p. H1013-20.

69. Mayhan, W.G., et al., Inhibition of $N A D(P) H$ oxidase alleviates impaired NOS-dependent responses of pial arterioles in type 1 diabetes mellitus. Microcirculation, 2006. 13(7): p. 567-75. 70. Miller, A.A., K. Budzyn, and C.G. Sobey, Vascular dysfunction in cerebrovascular disease: mechanisms and therapeutic intervention. Clin Sci (Lond), 2010. 119(1): p. 1-17.

71. Olmez, I. and H. Ozyurt, Reactive oxygen species and ischemic cerebrovascular disease. Neurochem Int, 2012. 60(2): p. 208-12.

72. Arrick, D.M., et al., Losartan improves impaired nitric oxide synthase-dependent dilatation of cerebral arterioles in type 1 diabetic rats. Brain Res, 2008. 1209: p. 128-35.

73. Banasiak, K.J., Y. Xia, and G.G. Haddad, Mechanisms underlying hypoxia-induced neuronal apoptosis. Prog Neurobiol, 2000. 62(3): p. 215-49.

74. Faraci, F.M., Protecting against vascular disease in brain. Am J Physiol Heart Circ Physiol, 2011. 300(5): p. H1566-82.

75. Iadecola, C., Neurovascular regulation in the normal brain and in Alzheimer's disease. Nat Rev Neurosci, 2004. 5(5): p. 347-60.

76. Iadecola, C., The pathobiology of vascular dementia. Neuron, 2013. 80(4): p. 844-66.

77. Frisbee, J.C., et al., Distinct temporal phases of microvascular rarefaction in skeletal muscle of obese Zucker rats. Am J Physiol Heart Circ Physiol, 2014. 307(12): p. H1714-28. 
78. Girouard, H., et al., Cerebrovascular nitrosative stress mediates neurovascular and endothelial dysfunction induced by angiotensin II. Arterioscler Thromb Vasc Biol, 2007. 27(2): p. 303-9.

79. Bowman, R.E., K.D. Beck, and V.N. Luine, Chronic stress effects on memory: sex differences in performance and monoaminergic activity. Horm Behav, 2003. 43(1): p. 48-59.

80. Lambert, G., et al., Reduced brain norepinephrine and dopamine release in treatmentrefractory depressive illness: evidence in support of the catecholamine hypothesis of mood disorders. Arch Gen Psychiatry, 2000. 57(8): p. 787-93.

81. Roy, A. and M.K. Campbell, A unifying framework for depression: bridging the major biological and psychosocial theories through stress. Clin Invest Med, 2013. 36(4): p. E170-90. 82. Zhang, Y., et al., Early Exercise Protects the Blood-Brain Barrier from Ischemic Brain Injury via the Regulation of MMP-9 and Occludin in Rats. Int J Mol Sci, 2013. 14(6): p. 11096112.

83. Beauquis, J., et al., Hippocampal neurovascular and hypothalamic-pituitary-adrenal axis alterations in spontaneously type 2 diabetic GK rats. Exp Neurol, 2010. 222(1): p. 125-34.

84. Dalla, C., et al., Sex differences in the effects of two stress paradigms on dopaminergic neurotransmission. Physiol Behav, 2008. 93(3): p. 595-605.

85. Winocur, G., et al., Memory impairment in obese Zucker rats: an investigation of cognitive function in an animal model of insulin resistance and obesity. Behav Neurosci, 2005. 119(5): p. 1389-95.

86. Chrissobolis, S. and F.M. Faraci, The role of oxidative stress and NADPH oxidase in cerebrovascular disease. Trends Mol Med, 2008. 14(11): p. 495-502.

87. Ergul, A., et al., Cerebrovascular complications of diabetes: focus on stroke. Endocr Metab Immune Disord Drug Targets, 2012. 12(2): p. 148-58.

88. Bedard, K. and K.H. Krause, The NOX family of ROS-generating NADPH oxidases: physiology and pathophysiology. Physiol Rev, 2007. 87(1): p. 245-313.

89. Springo, Z., et al., Aging Exacerbates Pressure-Induced Mitochondrial Oxidative Stress in Mouse Cerebral Arteries. J Gerontol A Biol Sci Med Sci, 2015. 70(11): p. 1355-9.

90. Tucsek, Z., et al., Obesity in aging exacerbates blood-brain barrier disruption, neuroinflammation, and oxidative stress in the mouse hippocampus: effects on expression of 
genes involved in beta-amyloid generation and Alzheimer's disease. J Gerontol A Biol Sci Med Sci, 2014. 69(10): p. 1212-26.

91. Tucsek, Z., et al., Aging exacerbates obesity-induced cerebromicrovascular rarefaction, neurovascular uncoupling, and cognitive decline in mice. J Gerontol A Biol Sci Med Sci, 2014. 69(11): p. 1339-52.

92. Pretnar-Oblak, J., et al., Influence of atorvastatin treatment on L-arginine cerebrovascular reactivity and flow-mediated dilatation in patients with lacunar infarctions. Stroke, 2006. 37(10): p. 2540-5. 


\section{FIGURE LEGENDS}

Figure 1: MCA vasodilation to acetylcholine in LZR and OZR following 8 weeks of chronic stress. Dose-response dilation to ACh for LZR control, LZR UCMS, OZR control, and OZR UCMS (Panel A). Maximum dilation to ACh to each group (Panel B). Dilation to ACh for LZR control and LZR UCMS after acute inbubation of vessels with TEMPOL or LNAME (Panel C). Dilation to ACh for OZR control and OZR UCMS after acute inbubation of vessels with TEMPOL or LNAME (Panel D). All data are presented as mean \pm SE. * $\mathrm{p}<0.05$ versus responses in LZR control at 17 weeks; $\uparrow \mathrm{p}<0.05$ versus responses in OZR control at 17 weeks. Please see text for more details and formulae.

Figure 2: Maximum dilation of MCA to ACh and SNP in LZR and OZR following 8 weeks of chronic stress. Maximum dilatory capacticity is shown for MCA to ACh, SNP, ACh after incubation with TEMPOL, and ACh after incubation with L-NAME for LZR control (Panel A), LZR UCMS (Panel B), OZR control (Panel C), and OZR UCMS (Panel D). All data are presented as mean $\pm \mathrm{SE}$. $* \mathrm{p}<0.05$ versus responses in LZR control at 17 weeks; $\uparrow \mathrm{p}<0.05$ versus responses in OZR control at 17 weeks. Please see text for more details and formulae.

Figure 3: Effects of 8 weeks of exercise training on vasodilatory responses to ACh and SNP in LZR and OZR. Dose-response dilation to ACh for LZR control, LZR UCMS, LZR Exercise, and LZR UCMS+Exercise (Panel A). Dose-response dilation to ACh for OZR control, OZR UCMS, OZR Exercise, and OZR UCMS+Exercise (Panel B). Maximal dilation to SNP for LZR control, LZR UCMS, LZR Exercise, and LZR UCMS+Exercise (Panel C). Maximal dilation to SNP for OZR control, OZR UCMS, OZR Exercise, and OZR UCMS+Exercise (Panel D). All data are

presented as mean $\pm \mathrm{SE} . * \mathrm{p}<0.05$ versus responses in $\mathrm{LZR}$ control at 17 weeks; $\uparrow \mathrm{p}<0.05$ versus responses in OZR control at 17 weeks. Please see text for more details and formulae. 
Figure 4: Effects of 8 weeks of exercise training on eutrophic remodeling of MCA in LZR and OZR. Passive inner diameter as measured after incubation of vessel in a $\mathrm{Ca}^{++}$-free physiological salt solution for LZR control, LZR UCMS, LZR Exercise, and LZR UCMS+Exercise (Panel A). Passive inner diameter as measured after incubation of vessel in a $\mathrm{Ca}^{++}$-free physiological salt solution for OZR control, OZR UCMS, OZR Exercise, and OZR UCMS+Exercise (Panel B). Vessel wall thickness as measured after incubation of vessel in a $\mathrm{Ca}^{++}$-free physiological salt solution for LZR control, LZR UCMS, LZR Exercise, and LZR UCMS+Exercise (Panel C). Vessel wall thickness as measured after incubation of vessel in a $\mathrm{Ca}^{++}$-free physiological salt solution for OZR control, OZR UCMS, OZR Exercise, and OZR UCMS+Exercise (Panel D). All data are presented as mean $\pm \mathrm{SE} . * \mathrm{p}<0.05$ versus responses in LZR control at 17 weeks; $\dagger$ $\mathrm{p}<0.05$ versus responses in OZR control at 17 weeks. Please see text for more details and formulae.

Figure 5: Maximal Dilation of MCA to ACh from OZR control and OZR after 8 weeks of exercise training. Dilation also shown for vessel after incubation in TEMPOL or L-NAME. All data are presented as mean $\pm \mathrm{SE}$. $* \mathrm{p}<0.05$ versus responses in OZR control at 17 weeks. Please see text for more details and formulae.

Figure 6: Maximum Dilation of MCAs in Exercise-trained LZR and OZR after 8 weeks of chronic stress. Maximal dilation to ACh, SNP, and ACh in the presence of TEMPOL or LNAME for LZR UCMS Exercise (Panel A). Maximal dilation to ACh, SNP, and ACh in the presence of TEMPOL or L-NAME for OZR UCMS Exercise (Panel B). All data are presented as mean \pm SE. $* p<0.05$ versus responses in LZR control at 17 weeks; $\uparrow \mathrm{p}<0.05$ versus responses in OZR control at 17 weeks. Please see text for more details and formulae 
Figure 7: Maximal dilation of MCA to ACh in exercise trained LZR and OZR after 8 weeks of UCMS based on exercise response. Maximum dilatory capacity is shown for MCA to ACh for OZR control, OZR exercise, OZR exercise Good Responders, and OZR exercise Poor Responders (Panel A). Maximum dilatory capacity is shown for MCA to ACh for OZR control, OZR UCMS, OZR UCMS + Exercise, OZR UCMS + Exercise Good Responders, and OZR UCMS + Exercise Poor Responders (Panel B).Maximum dilatory capacity is shown for MCA to ACh for LZR control, LZR UCMS, LZR UCMS + Exercise, LZR UCMS + Exercise Good Responders, and LZR UCMS + Exercise Poor Responders (Panel C). All data are presented as mean \pm SE. $* \mathrm{p}<0.05$ versus responses in LZR control at 17 weeks; $\uparrow \mathrm{p}<0.05$ versus responses in OZR control at 17 weeks. Please see text for more details and formulae

Figure 8: Vasoconstriction to 5-HT in LZR and OZR after 8 weeks of exercise training and UCMS. Dose-response constriction to 5-HT for LZR control, LZR UCMS, LZR Exercise, and LZR UCMS+Exercise (Panel A). Dose-response constriction to 5-HT for OZR control, OZR UCMS, OZR Exercise, and OZR UCMS+Exercise (Panel B). Maximal constriction to 5-HT for LZR control, LZR UCMS, LZR Exercise, and LZR UCMS+Exercise (Panel C). Maximal constriction to 5-HT for OZR control, OZR UCMS, OZR Exercise, and OZR UCMS+Exercise (Panel D). All data are presented as mean \pm SE. $* \mathrm{p}<0.05$ versus responses in LZR control at 17 weeks; $\uparrow \mathrm{p}<0.05$ versus responses in OZR control at 17 weeks. Please see text for more details and formulae

Figure 9: Cerebral microvessel density and protein expression of CD-31 and VEGF in the cortex and striatum of LZR and OZR following 8 weeks of exercise and UCMS. Semi-quantitative analysis of microvessel density by immunostain for anti-CD-31 in the cortex of LZR and OZR control, LZR and OZR UCMS, LZR and OZR Exercise, and LZR and OZR UCMS + Exercise 
(Panel A). Semi-quantitative analysis of microvessel density by immunostain for anti-CD-31 in the striatum of LZR and OZR control, LZR and OZR UCMS, LZR and OZR Exercise, and LZR and OZR UCMS + Exercise (Panel B). Western blot quantification of CD-31 protein expression in the brains of LZR and OZR control, LZR and OZR UCMS, LZR and OZR Exercise, and LZR and OZR UCMS + Exercise (Panel C). Western blot quantification of VEGF protein expression in the brains of LZR and OZR control, LZR and OZR UCMS, LZR and OZR Exercise, and LZR and OZR UCMS + Exercise (Panel C). All data are presented as mean \pm SE. $* \mathrm{p}<0.05$ versus responses in LZR control at 17 weeks; $\uparrow \mathrm{p}<0.05$ versus responses in OZR control at 17 weeks. Please see text for more details and formulae

Supplemental Figures: Data shown for average mean arterial pressure at 17 weeks of age as measured by flow transducer placed into cannulated carotid artery (Supplemental Figure 1). Data shown for average weekly food intake by group over 8 weeks (Supplemental Figure 2). Data shown for average amount of work performed by each during exercise training (Supplemental Figure 3). Data shown for average weekly body mass for each group of the 8 week time course of these experiments (Supplemental Figure 4). 


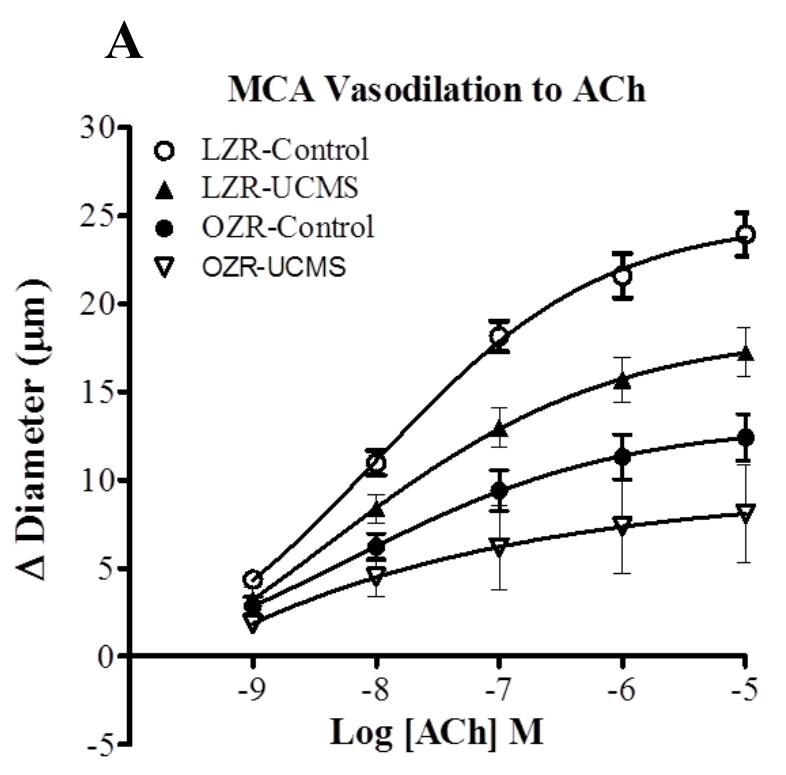

B

Maximum Dilation to $\mathrm{ACh}\left[10^{-5}\right] \mathrm{M}$

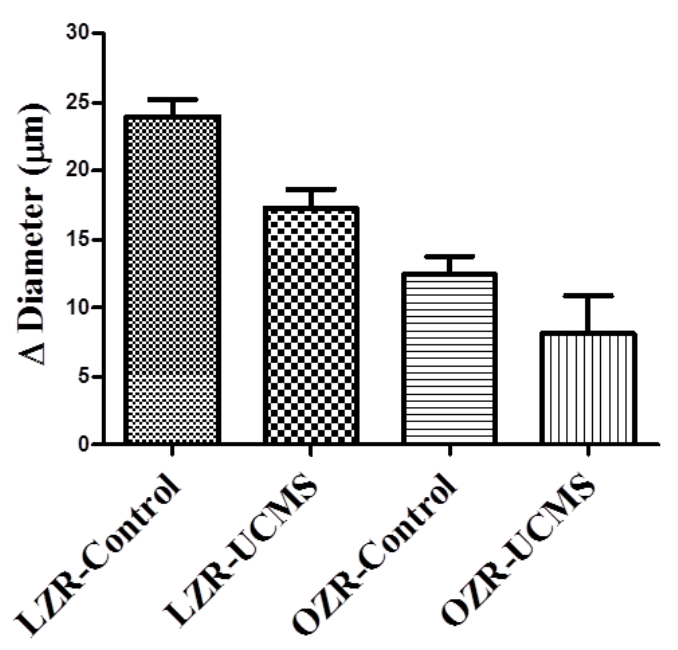

C
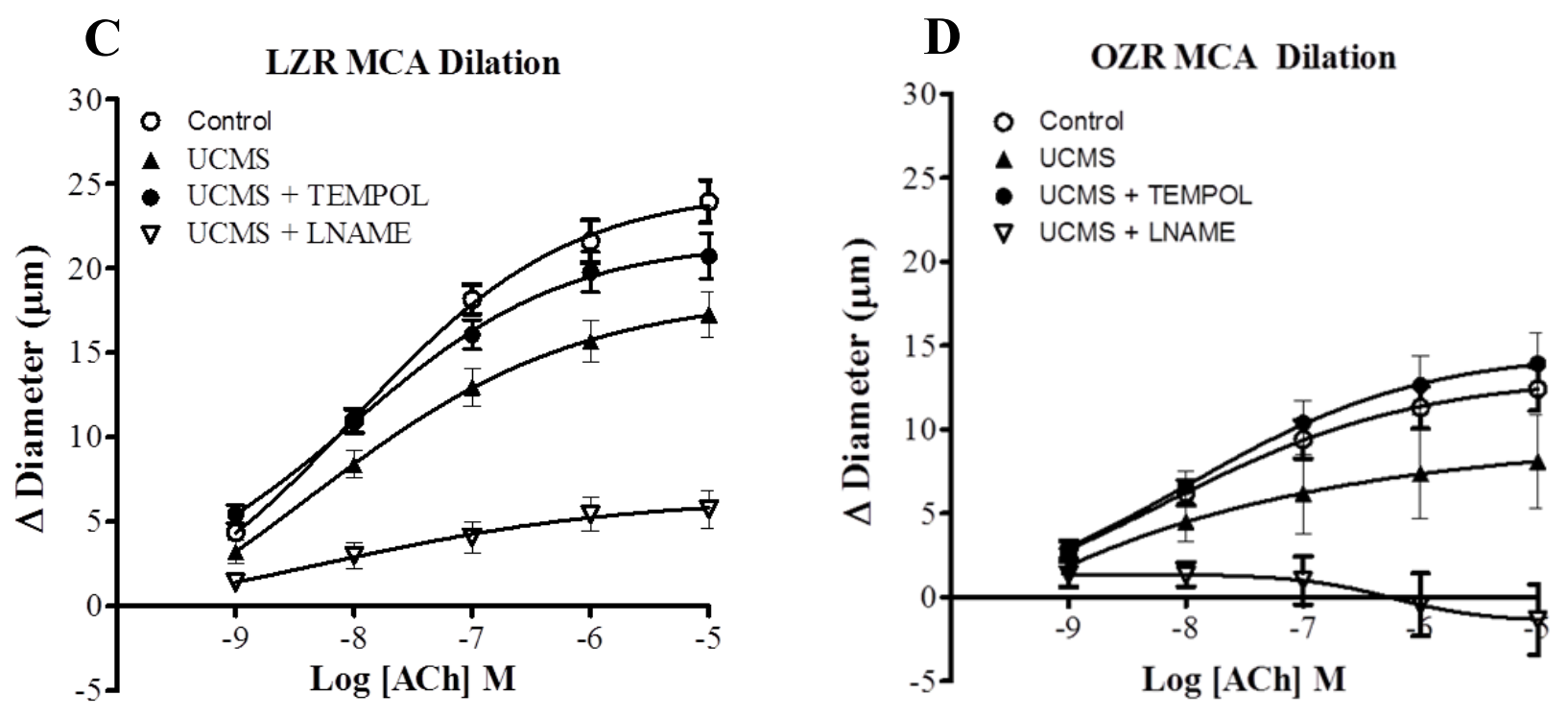

Figure 1 (MCA Dilation in LZR and OZR after 8 Weeks of UCMS) Brooks et al 
A

LZR Control Max Dilation

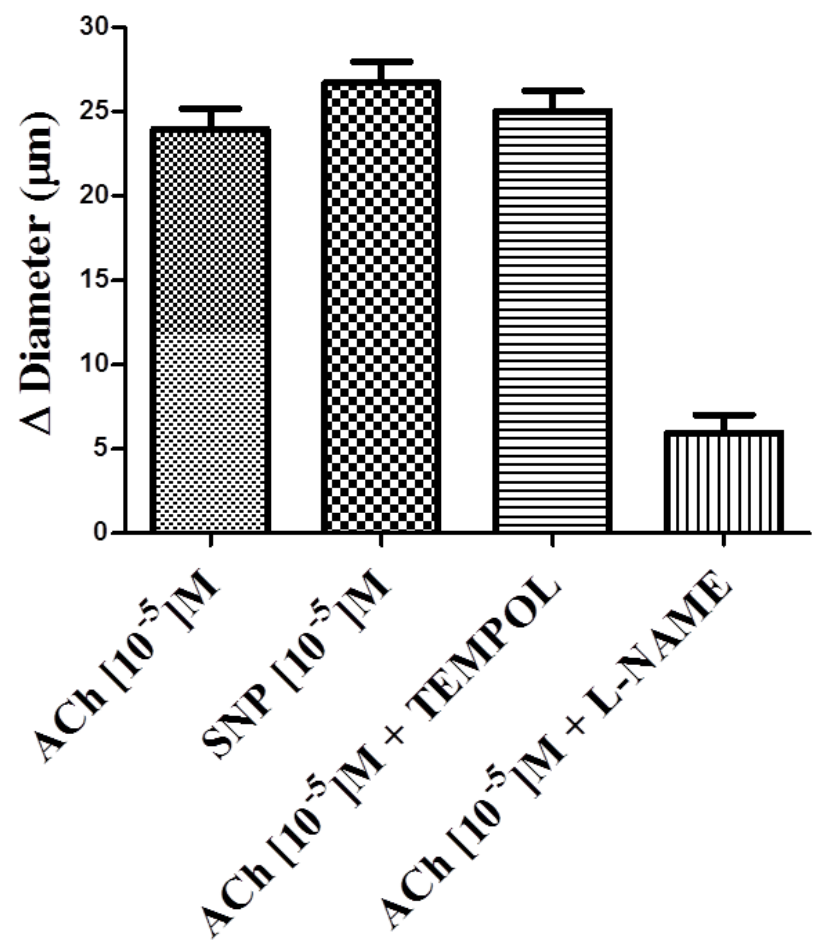

C

OZR Control Max Dilation
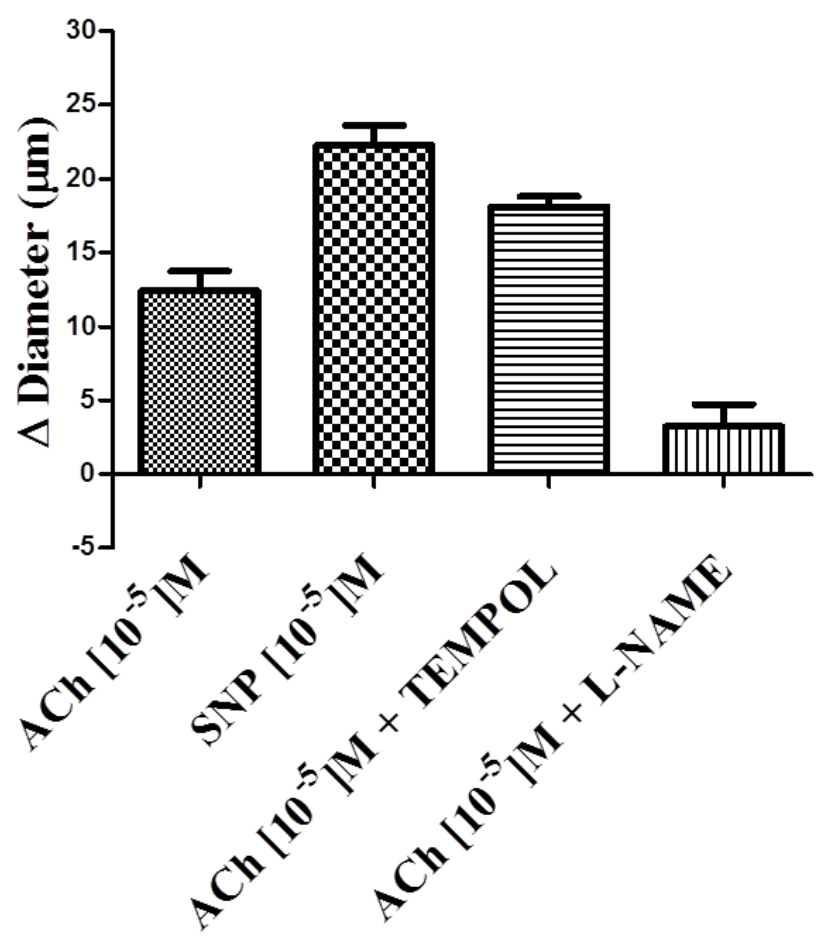

B

LZR UCMS Max Dilation
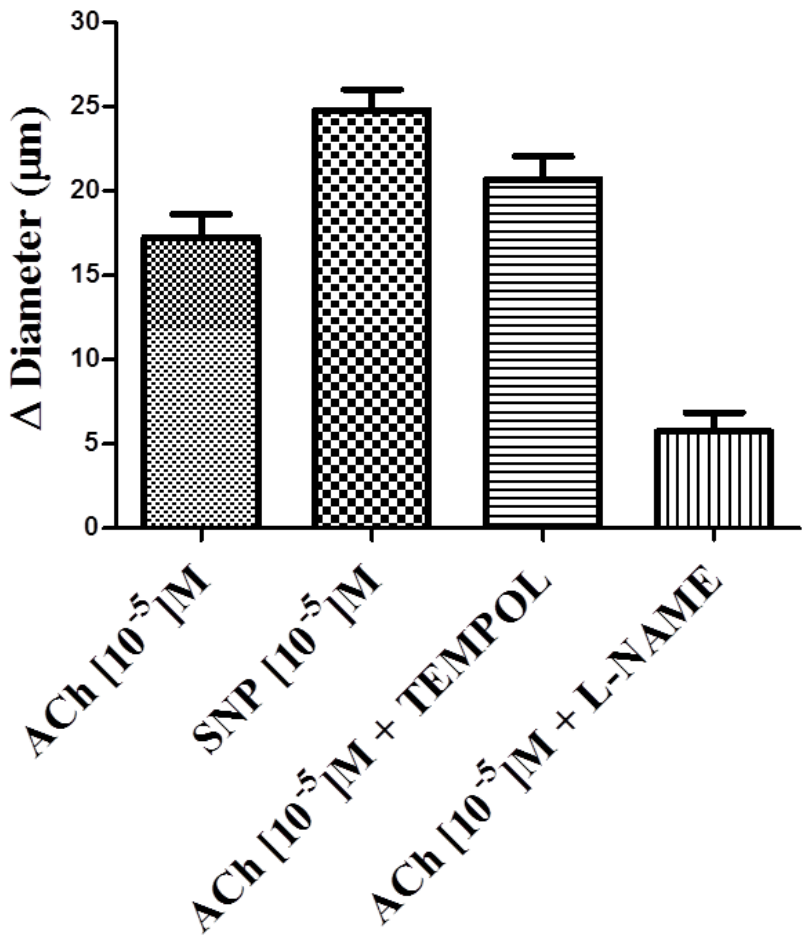

D

OZR UCMS Max Dilation

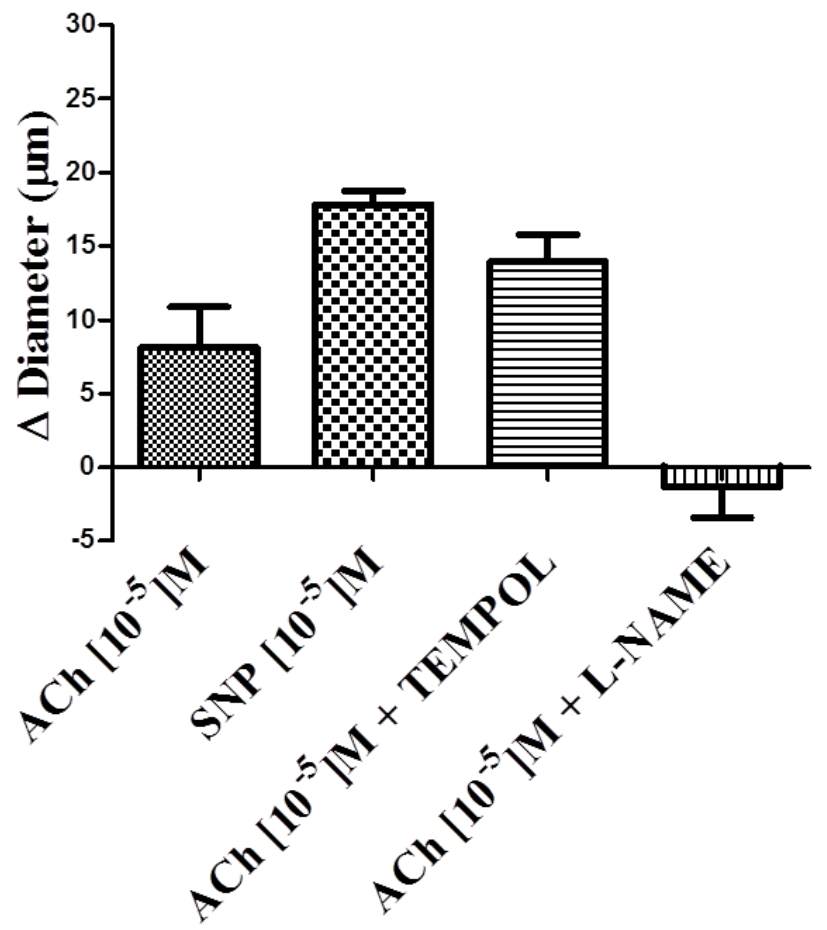

Figure 2 (Maximum Dilation of MCA)

Brooks et al 

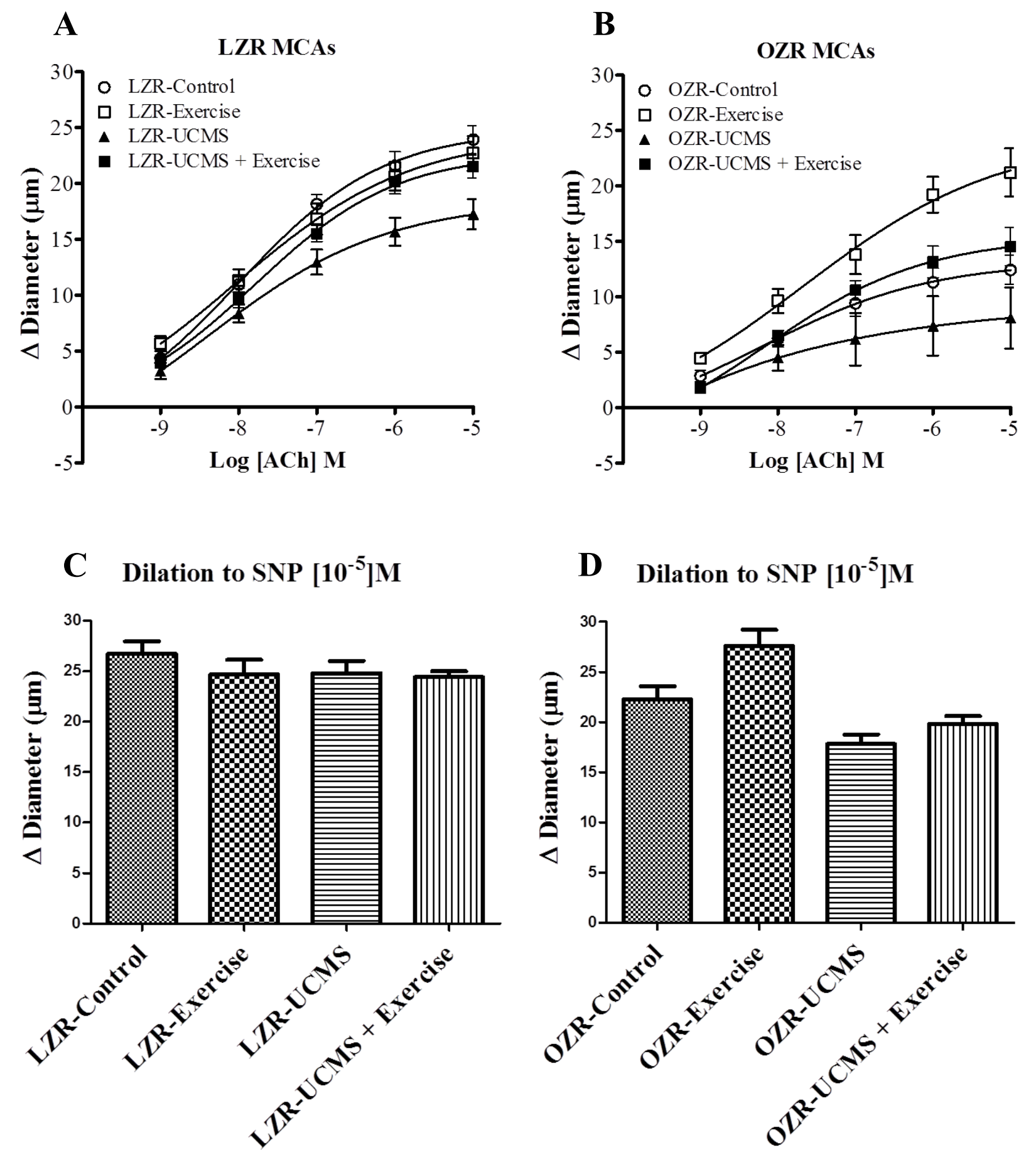

Figure 3 (Effect of Exercise on Dilation of MCA following UCMS) Brooks et al 
A

LZR Passive Inner Diameter
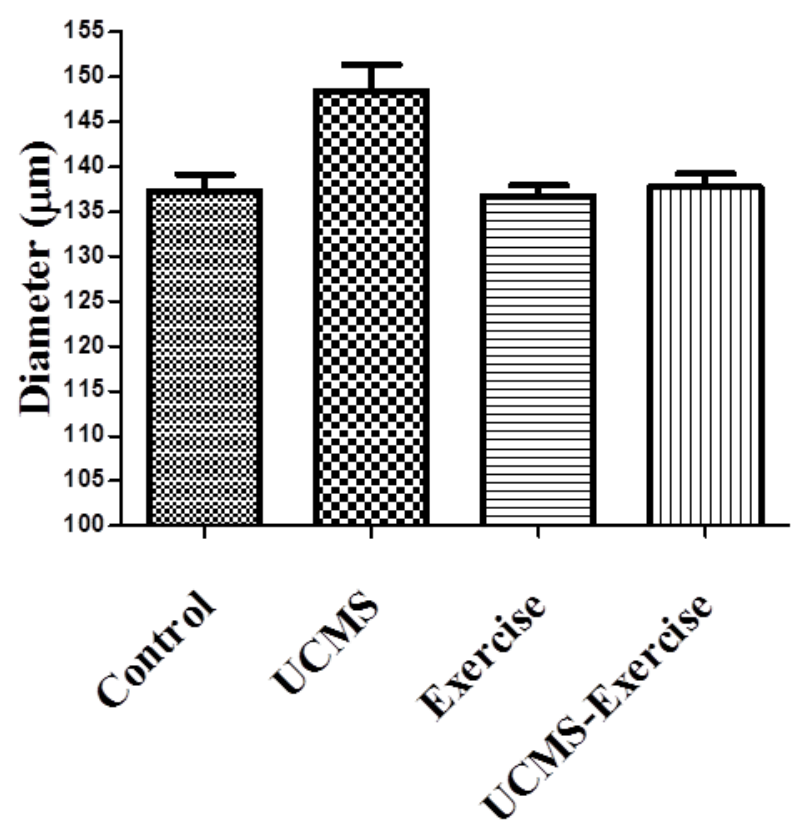

C

LZR Wall Thickness
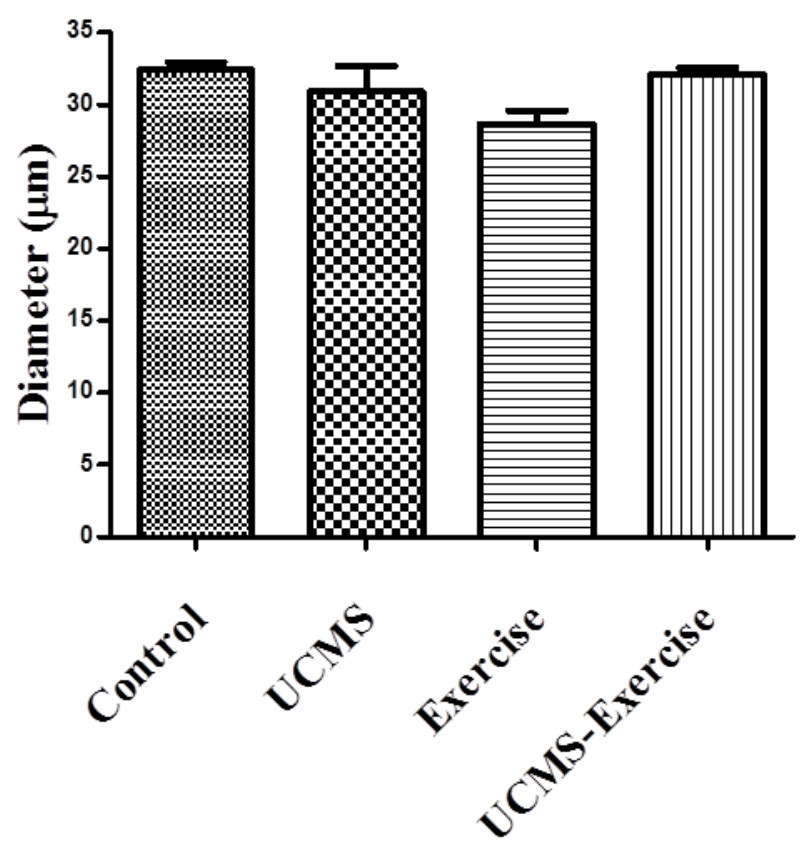

B

OZR Passive Inner Diameter

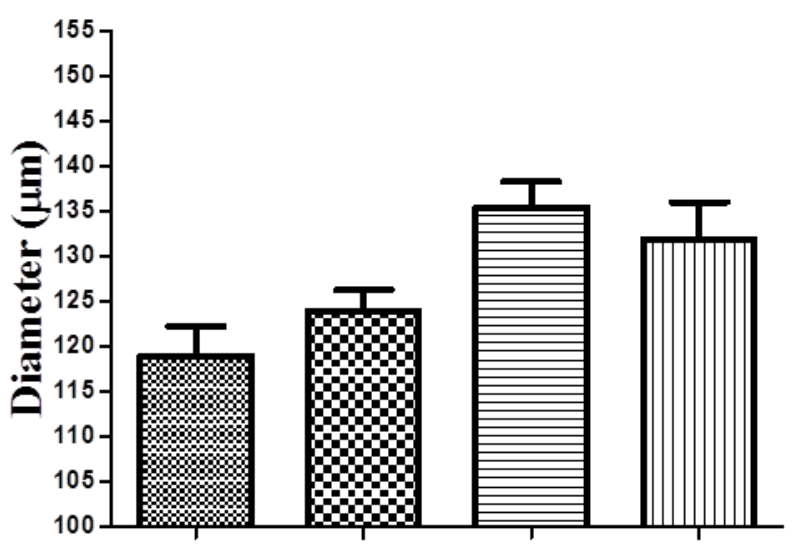

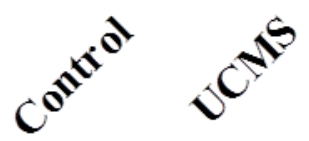

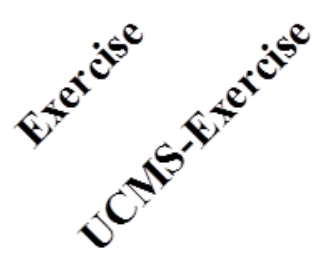

D

OZR Wall Thickness
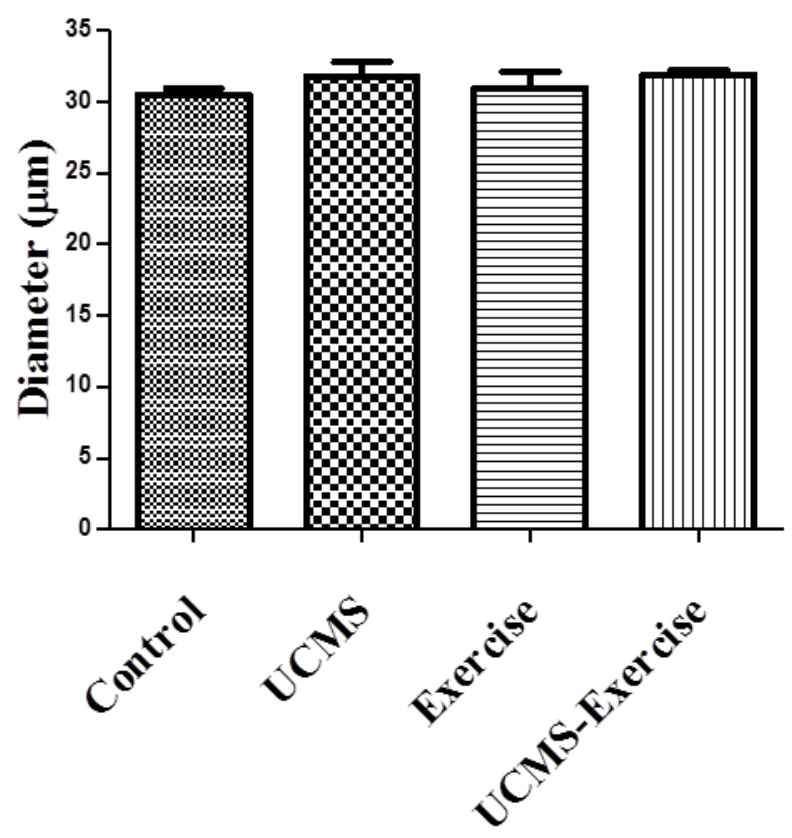

Figure 4 (Effect of Exercise on Eutrophic Remodeling of MCA)

Brooks et al 


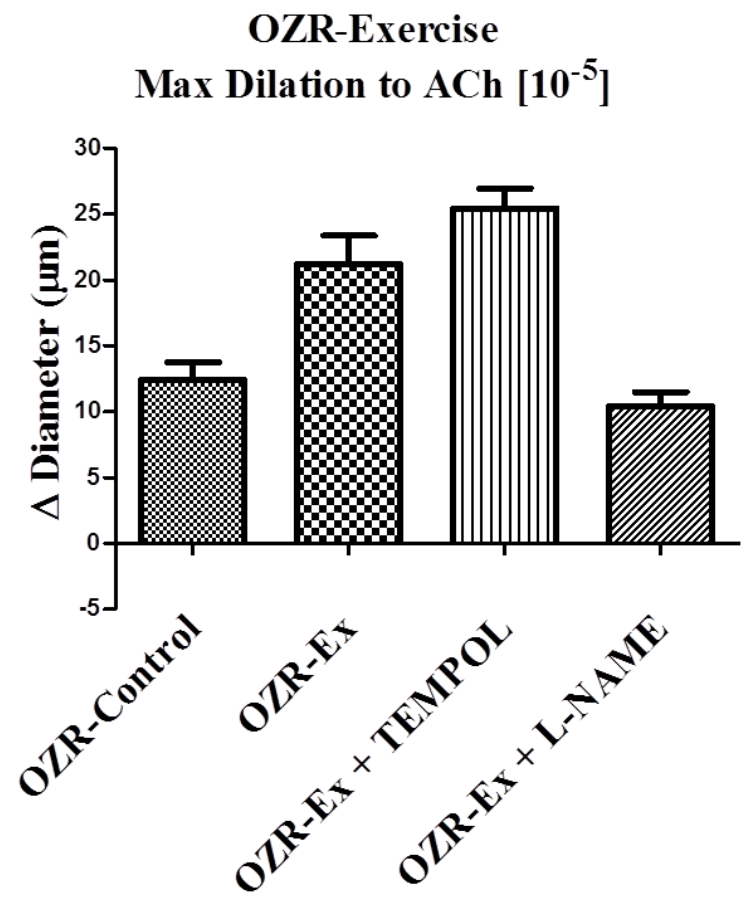

Figure 5 (Maximum Dilation of MCA in Exercise-trained OZR) Brooks et al 

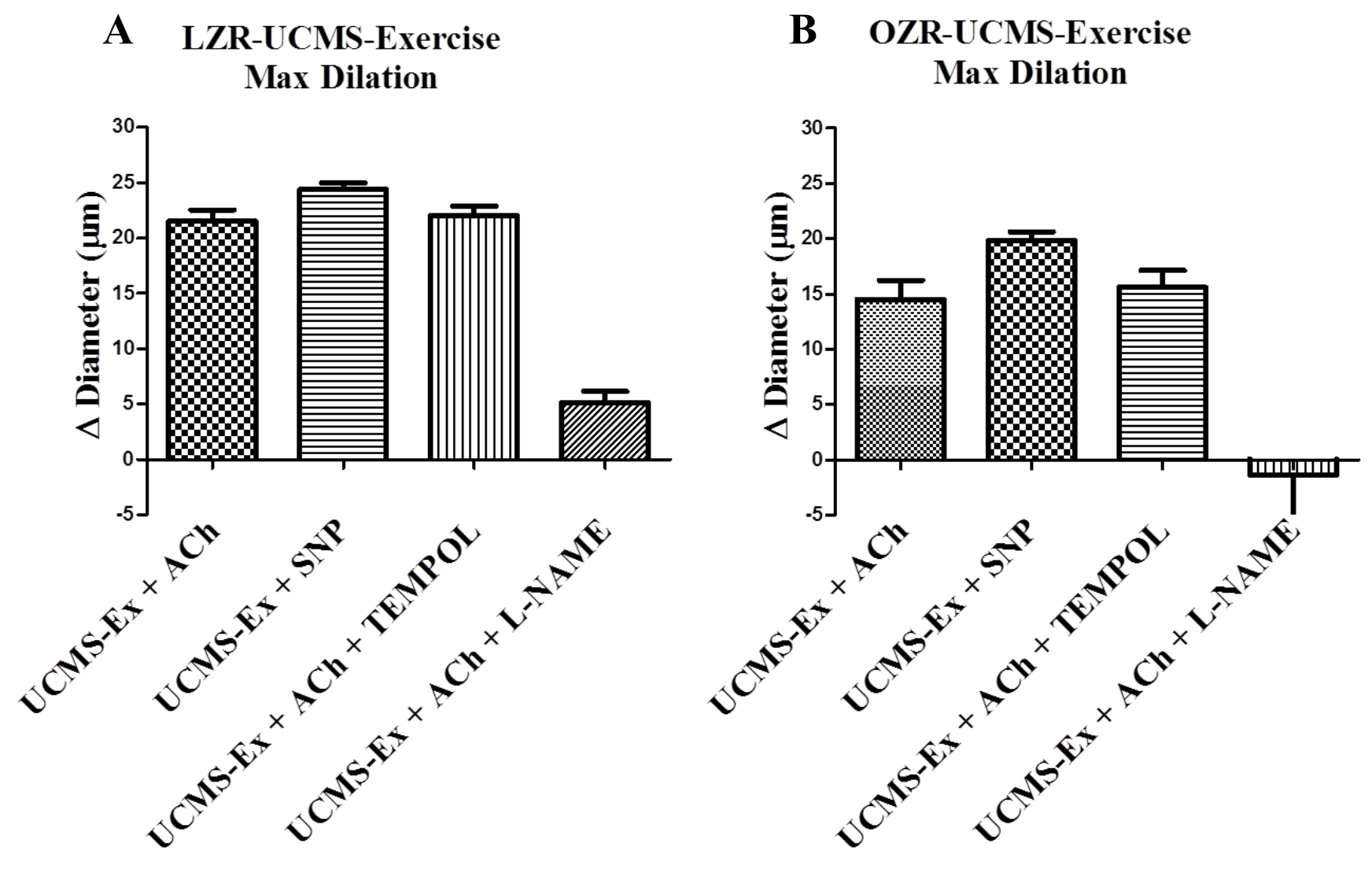

Figure 6 (Maximum Dilation of MCAs in Exercise-trained UCMS Animals) Brooks et al 
A OZR: Good vs Poor

Response to Exercise

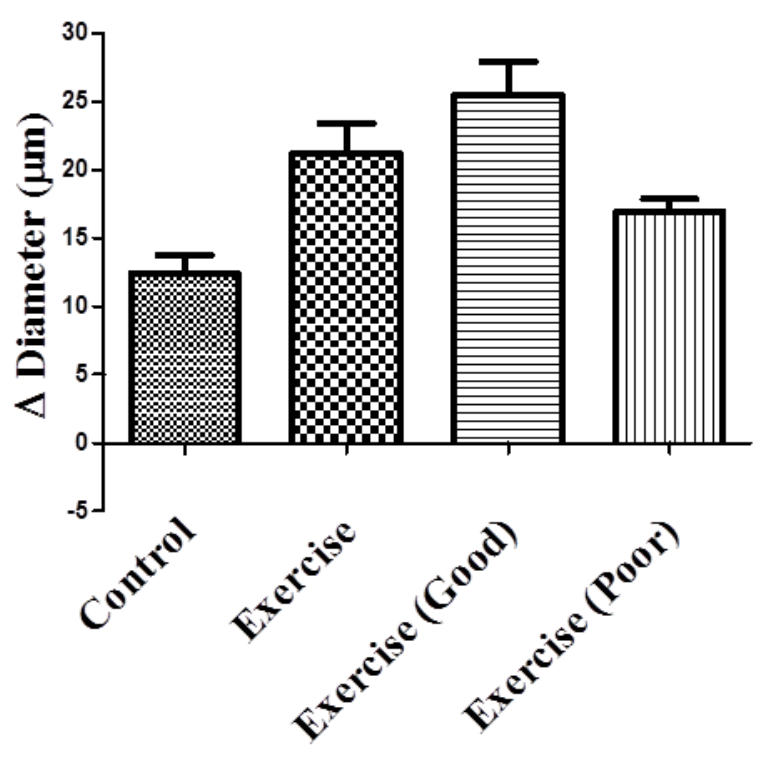

B OZR UCMS: Good vs Poor

Response to Exercise

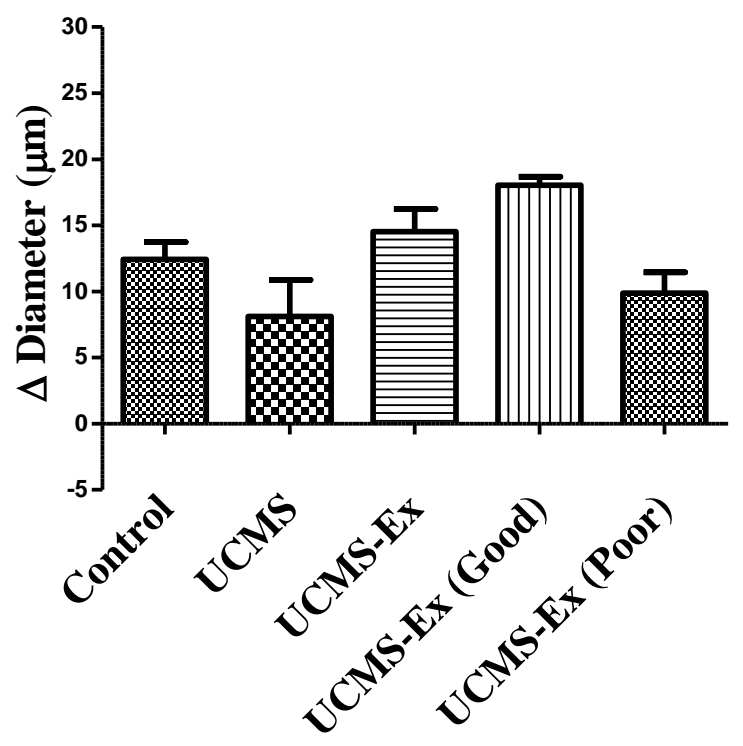

C LZR UCMS: Good vs Poor

Response to Exercise

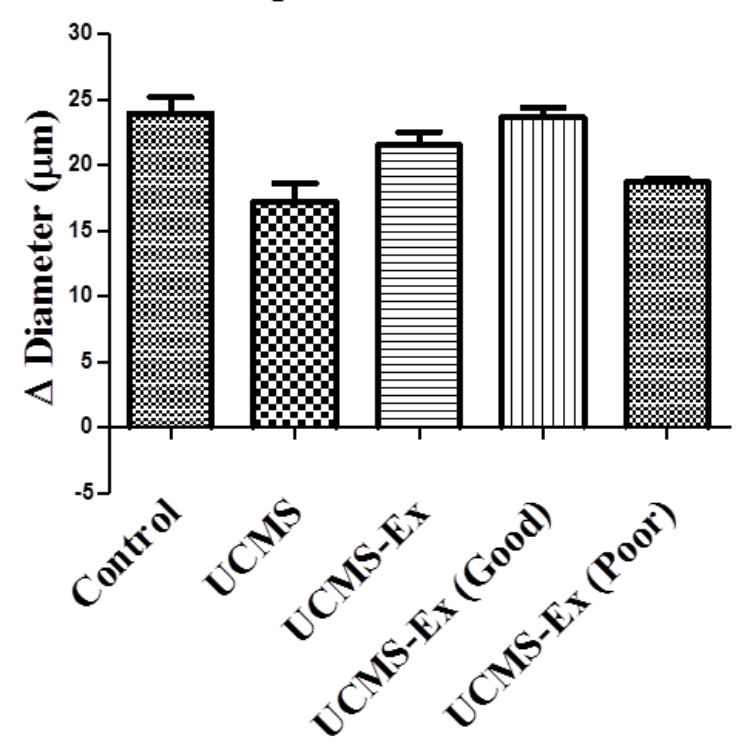

Figure 7 (Differential Responses of Good vs Poor Responders to Exercise Training) Brooks et al 

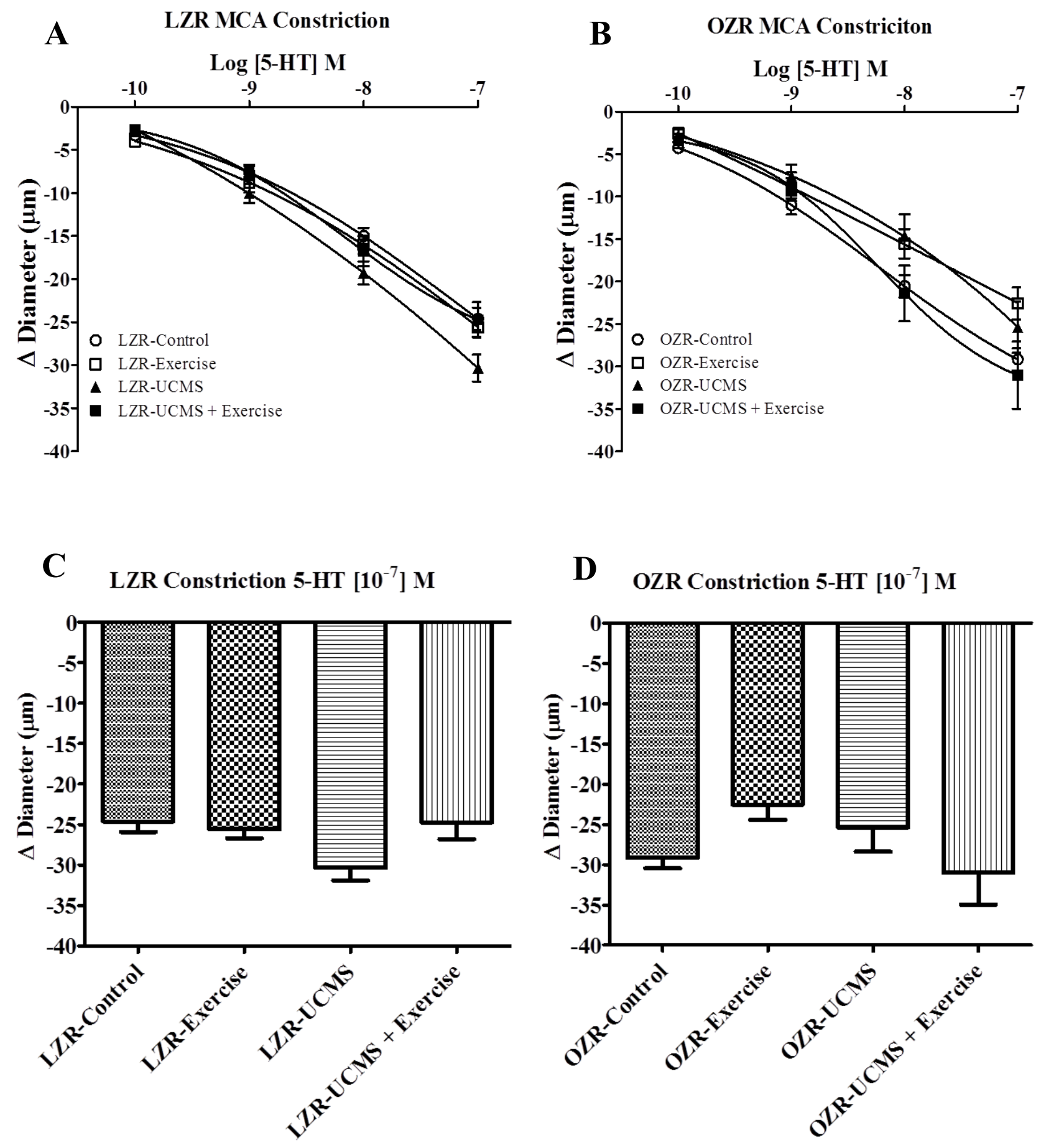

Figure 8 (MCA Constriction Responses Following UMCS) Brooks et al 
A
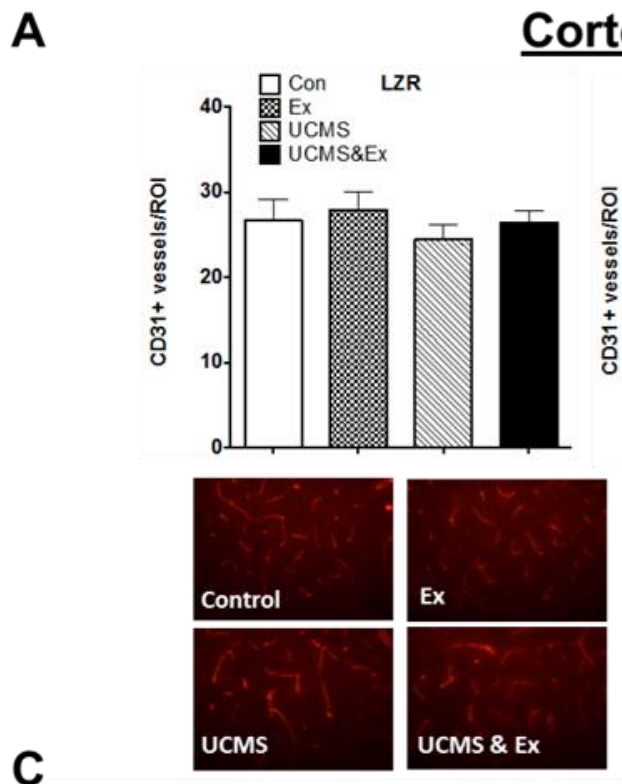

C

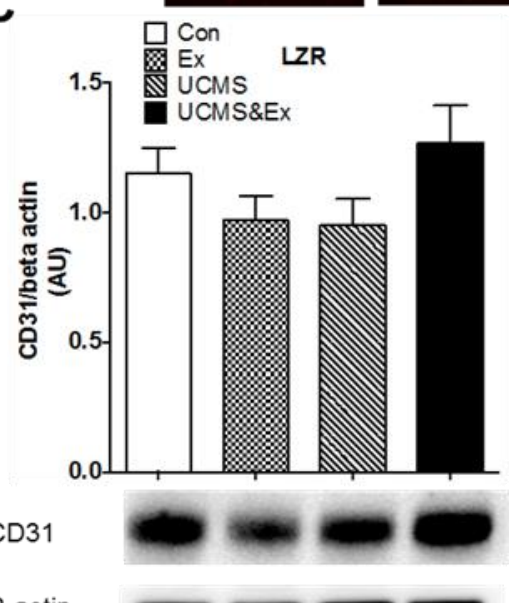

Cortex
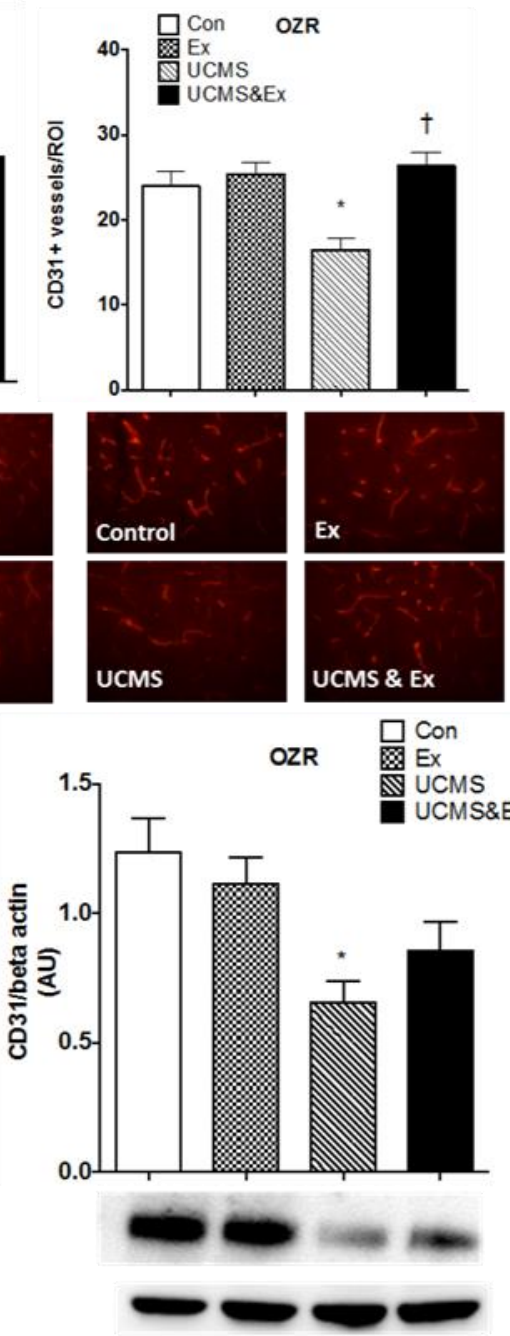

B

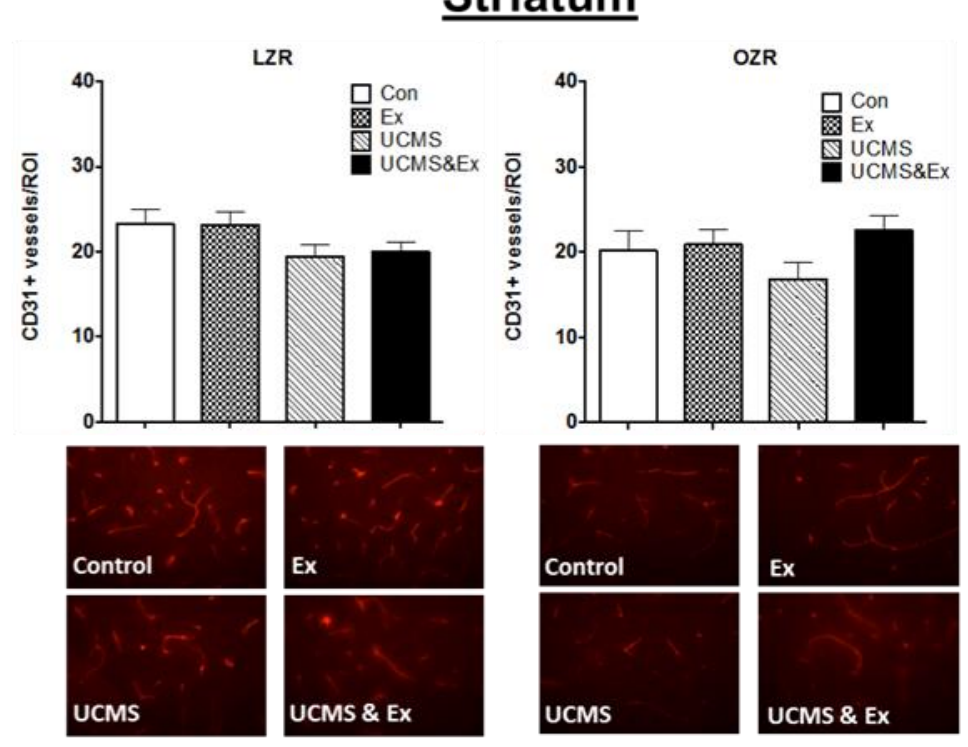

D

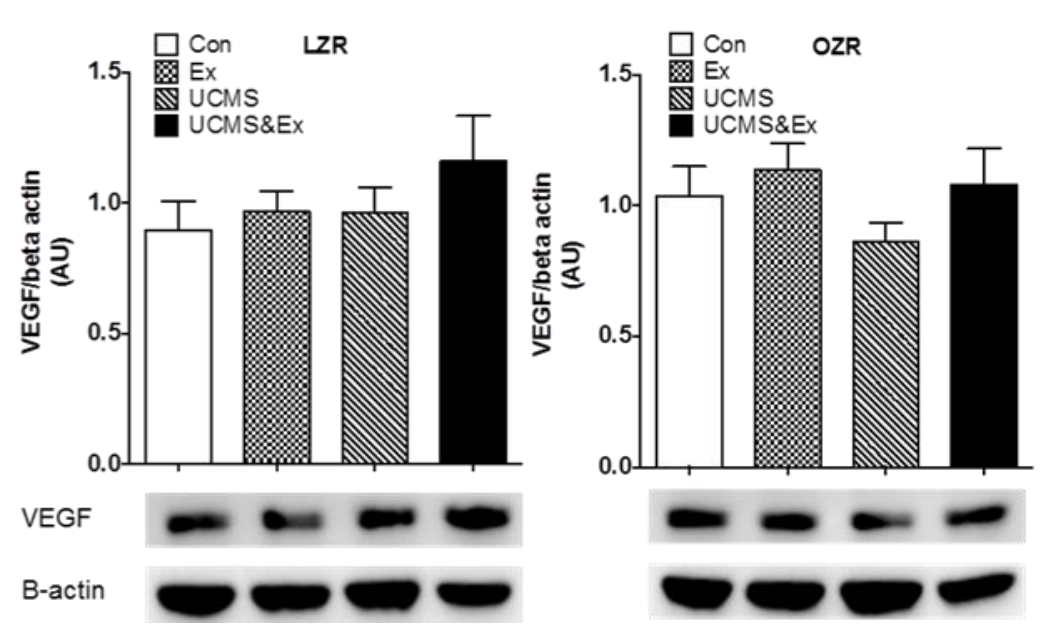

Figure 9 (Cerebrovascular Microvessel Density)

Brooks et al 


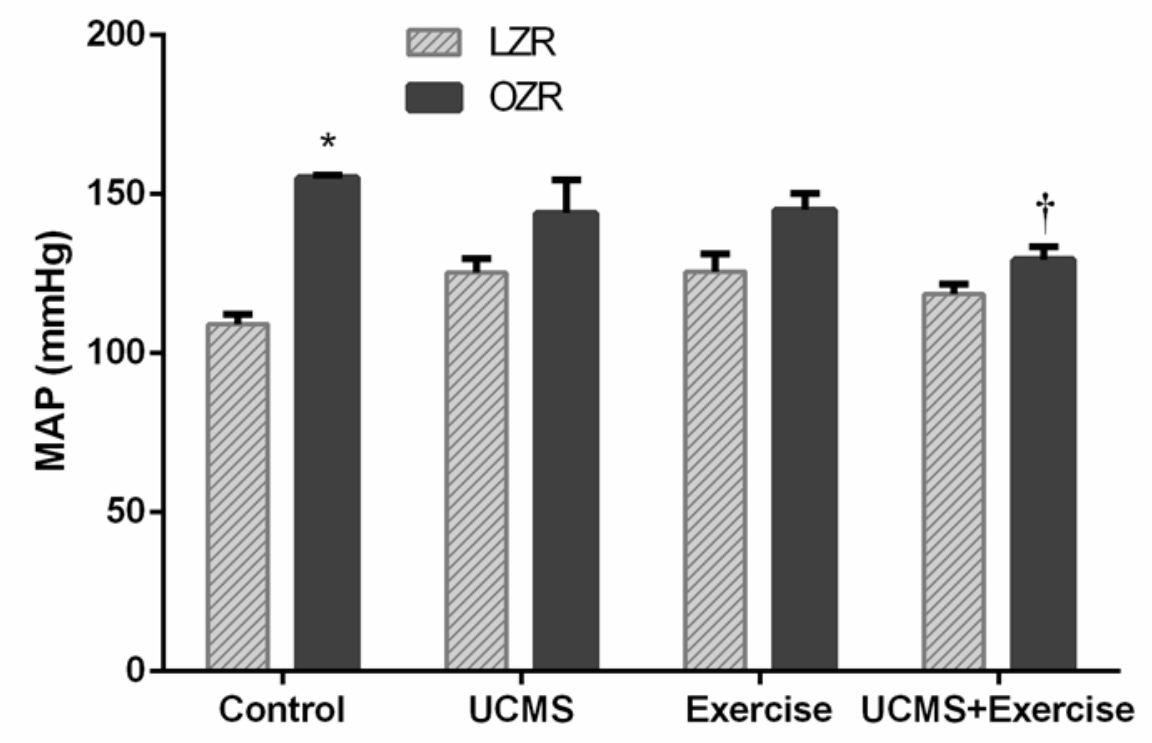

Supplemental Figure 1 (Mean Arterial Pressures at 17 Weeks) Brooks et al 


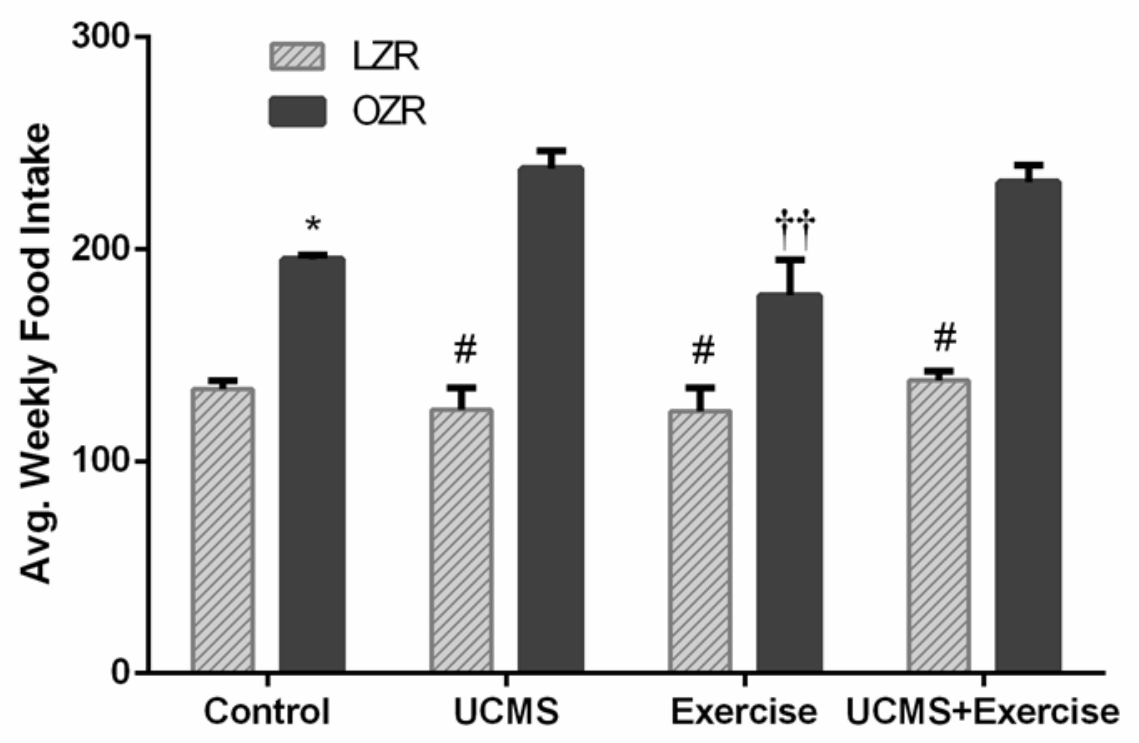

Supplemental Figure 2 (Average Weekly Food Intake)

Brooks et al 


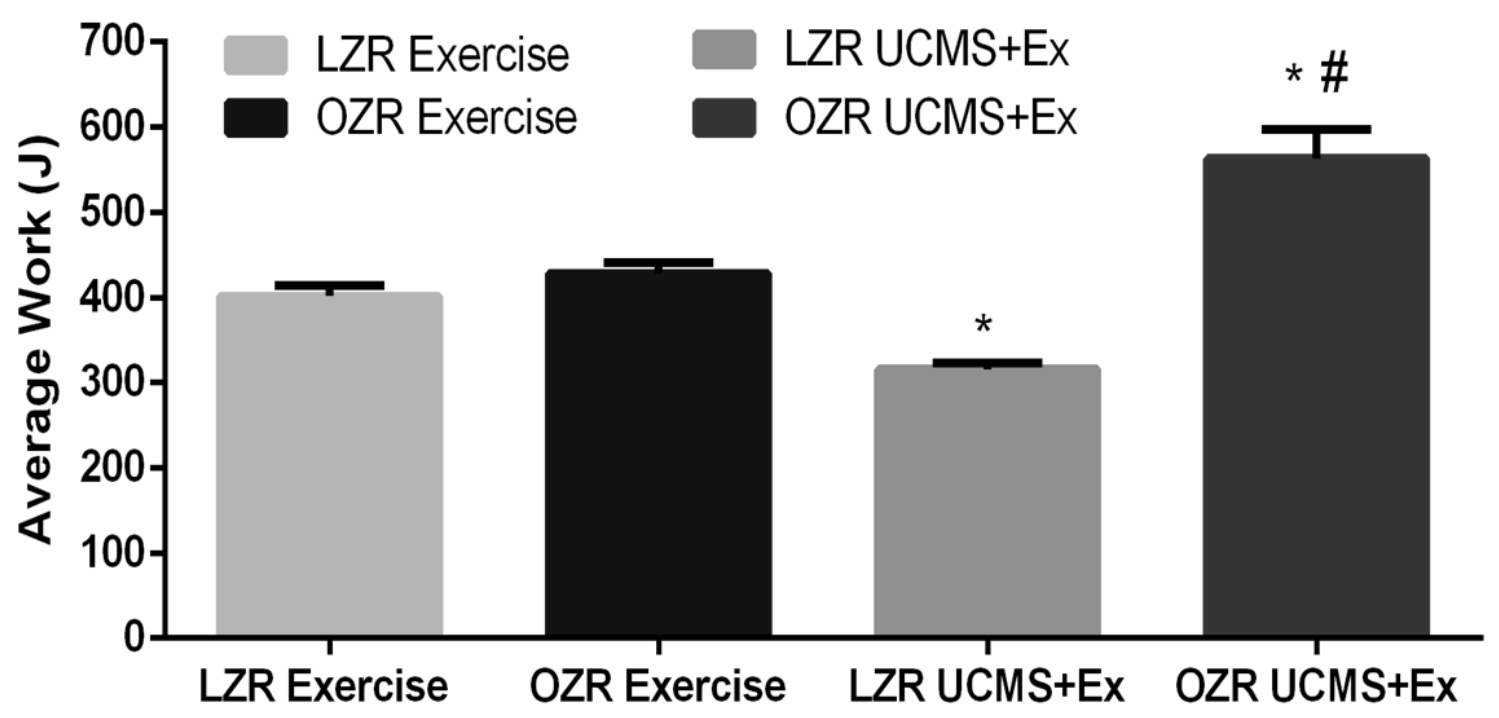

Supplemental Figure 3 (Average Work Performed During Exercise) Brooks et al 


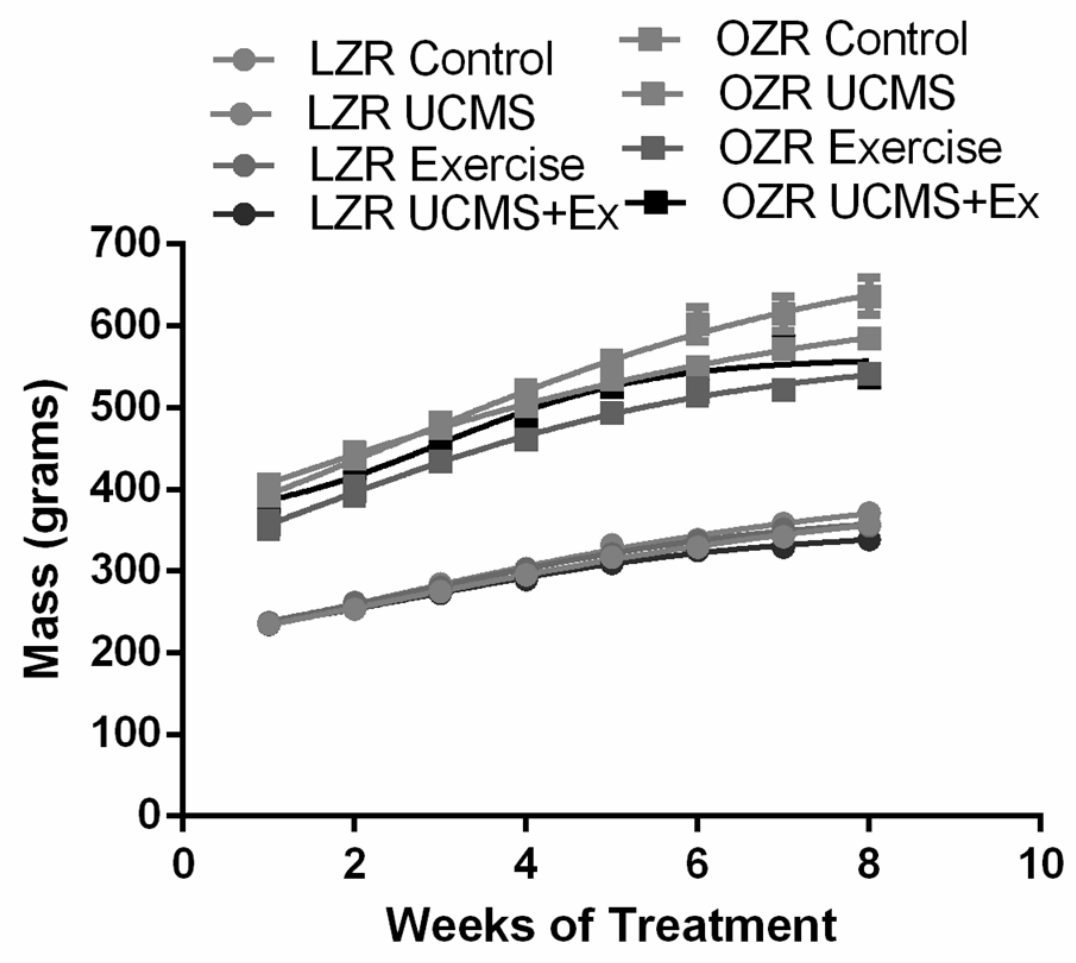

Supplemental Figure 4 (Change in Body Mass over 9 weeks of UCMS) Brooks et al 


\section{Chapter 5: Conceptual Framework}

This chapter investigated the effects of imposing the unpredictable chronic mild stress (UCMS) protocol on LZR and OZR for 8 weeks to induce depression and vascular dysfunction. A separate cohort of LZR and OZR received exercise training alone, or exercise training concurrent to UCMS, for the 8 week period. The main finding of this chapter is that UCMS induces vascular dysfunction in MCA of LZR and OZR, as well as eutrophic remodeling and cortical rarefaction in UCMS treated OZR. Acute incubation of vessels with TEMPOL and L-NAME revealed that these impairments largely stemmed from alterations in NO bioavailability, with impairments being substantially greater in OZR than LZR. Exercise training was able to improve each of these symptoms in both LZR and OZR. Interestingly, exercise trained animals from the control OZR and UCMS OZR groups, as well as the UCMS LZR groups, could be characterized as 'good' or 'poor' responders to exercise. 'Good' responding OZR showed a dramatic improvement in MCA reactivity, restoring reactivity to LZR control, while 'poor' responders showed a much smaller, albeit significant, improvement over OZR control and OZR UCMS. A similar dichotomy was seen for 'good' and 'poor' responders in LZR. These findings suggest that the anti-depressive and vasculoprotective benefits of exercise training may be limited by individual factors.

While the finding that exercise improves depressive symptoms and vascular health is not itself novel, this study was the first to conclusively link chronic stress/depression with cerebrovascular dysfunction, in lean as well as MetSyn animals, and the exercise component helps establish the mechanisms through which impairments occur and subsequently be treated. In sum, this chapter provides a final examination of functional impairments that result when comorbid metabolic syndrome is incorporated into a disease model. Considering the extremely high prevalence of MetSyn in the US, and its link to stroke, myocardial infarction, atherosclerosis, dementia, and Alzheimer's, it becomes necessary to question how the comorbid presence of MetSyn directly impacts the pathophysiology of these diseases. Incorporating MetSyn into preclinical models of these diseases may serve to enhance the translational utility of these models and improve the clinical relatability of their findings. 


\section{CHAPTER 6: DISSERTATION DISCUSSION}

The purpose of this dissertation was to define and interrogate the mechanisms of cerebrovascular dysfunction and remodeling that develop due to the underlying constituent pathologies of the metabolic syndrome (MetSyn), and to evaluate the functional consequences of these impairments on outcomes in ischemic stroke and stress-induced depression. Metabolic syndrome is one of the most costly and prevalent conditions in the United States, and its underlying pathological conditions- hypertension, hyperglycemia, insulin insensitivity, central obesity, and dyslipidemia- each directly contribute to mechanisms of vascular dysfunction and vascular remodeling. The obese Zucker rat (OZR) is a well-established model for studying the vascular complications of MetSyn, especially with respect to the peripheral circulation. Prior to these chapters written for this dissertation, however, very little was known about how MetSyn in the OZR specifically impacted the cerebrovascular circulation, and how these impairments would influence the physiologic responses to perturbations like ischemic stroke or stress-induced depression. Ischemic stroke in particular is highly correlated with MetSyn, yet few studies into the vascular responses to ischemic stroke have incorporated traditional stroke risk factors like MetSyn in their study design. After defining and investigating the mechanisms of cerebrovascular dysfunction in the OZR, this dissertation focuses specifically on how these impairments change or exacerbate pathophysiological outcomes in cerebrovascular disease.

The second chapter of this dissertation focused on quantifying the progressive decline in microvascular density in the frontal cortex of OZR, and utilized clinically relevant antihypertensives and glycemic control agents to assess the contribution of underlying hypertension and hyperglycemia/insulin resistance on the development of cerebral cortical rarefaction. Previous research from our laboratory and others had established that microvascular rarefaction 
occurs in the microcirculation of skeletal muscle, myocardium, and kidney. This study began by measuring microvascular density (MVD) in LZR and OZR at 7-8 weeks (when symptoms of MetSyn first begin to appear), 12-13 weeks, and 16-17 weeks (considered the age of full maturation of MetSyn), and documented a significant, progressive decline in cortical MVD in OZR. OZR were then chronically treated from 7 weeks of age with either an anti-hypertensive drug (captopril, an ACE inhibitor; hydralazine, a systemic vasodilator), or a glycemic control agent (metformin, hepatic gluconeogenesis inhibitor; rosiglitazone, insulin sensitizing agent), and evaluated for MVD at the same time points. A separate cohort of OZR was treated with TEMPOL to evaluate the effect of chronic anti-oxidant therapy on rarefaction, and/or L-NAME, an endothelial nitric oxide synthase (eNOS) inhibitor. These treatments were selected to improve NO bioavailability (TEMPOL), greatly reduce/inhibit NO bioavailability (L-NAME), and evaluate potential non-NO related benefits of anti-oxidant therapy (TEMPOL+L-NAME).

Neither captopril nor hydralazine was able to prevent cerebral cortical rarefaction at 17 weeks, although captopril did delay onset and reduced rarefaction by almost half. In contrast, both metformin and rosiglitazone significantly improved MVD at 17 weeks, preventing the vast majority of rarefaction. The preliminary conclusion suggested by results is that management of hyperglycemia and insulin resistance is critical to preventing cortical rarefaction. Treatment with TEMPOL, however, completely prevented rarefaction in OZR at all time points, rendering it as the most effective intervention. Treatment with L-NAME, both with and without TEMPOL, resulted in the same temporal progression of rarefaction observed in OZR control, suggesting that TEMPOL's therapeutic benefit lays solely in its ability to increase NO bioavailability. Mechanistically, these results suggest that management of oxidant load in the cerebral circulation is the most important factor towards mitigating cortical rarefaction. A large body of literature has 
linked both type 1 and type 2 diabetes to cerebrovascular dysfunction, and identified reactive oxygen species (ROS) such as superoxide as causative agents. Additionally, angiotensin receptor signaling in the brain is directly linked to endothelial generation of ROS, so systemic treatment with an ACE inhibitor like captopril should induce some reduction in oxidative stress. That TEMPOL was the most effective, and L-NAME eliminated $100 \%$ of its benefit, supports this hypothesis. The measurements of NO bioavailability further confirm this hypothesis, and a strong, linear relationship is evident between NO bioavailability and cerebral cortical MVD.

The second chapter of this dissertation establishes clearly that progression of MetSyn in ORZ lead to loss of NO bioavailability, with direct implications for MVD. It was therefore hypothesized that impairments in cerebrovascular reactivity, and alterations in cerebral vessel wall mechanics, would also develop in OZR. To test this hypothesis, the third chapter of this dissertation investigated the structural and functional changes in middle cerebral arteries (MCA) of OZR at 17 weeks, and utilized chronic treatment with pharmacological agonists targeted at each of the constituent pathologies of MetSyn (anti-hypertensive, anti-diabetic, antidyslipidemic) to investigate how each of these cardiovascular phenotypes individually impact the functional and structural alterations in the vasculature of the brain. Similar to Chapter 2, chronic TEMPOL treatment and L-NAME treatment were utilized to directly assess what effect antioxidant therapy and elimination of NO bioavailability have on the progression of each functional and structural outcome.

By 17 weeks of age, MCA of OZR had developed severe impairment to endotheliumdependent vasodilation, increased myogenic activation, significantly reduced NO bioavailability, eutrophic inward remodeling, and increased mechanical stiffening. Plasma levels of biomarkers for inflammation and oxidative stress were also elevated significantly. Stiffening of MCAs 
occurred earliest, and seemed to precede the other structural and functional deficits, and acute incubation of isolated MCA with TEMPOL rescues some, but not all, dilatory capability and NO bioavailability. Management of hypertension in OZR was most significantly related to changes in arterial stiffening. The two anti-hypertensives, hydralazine and captopril, effectively mitigated increase in arterial stiffness, as did atorvastatin and TEMPOL, which each independently caused a reduction in blood pressure. In this study, the anti-diabetic agent rosiglitazone helped reduce inward remodeling, and improved NO bioavailability and vasodilation to acetylcholine. Rosiglitazone was more effective than metformin at improving NO bioavailability, and was also more effective at improving vascular reactivity and reducing arterial stiffness. The effects of the anti-dyslipidemia drugs were highly divergent; while both atorvastatin and gemfibrozil effectively controlled plasma cholesterol levels, atorvastatin also conferred significant improvements in NO bioavailability, vascular reactivity, and vascular stiffness, while gemfibrozil did not. Atorvastatin is known to have anti-inflammatory and anti-oxidative pleiotropic actions, providing a potential explanation for the difference from gemfibrozil.

The most significant finding of this chapter was that interventions which increased NO bioavailability also significantly improved vascular reactivity, reduced inflammatory biomarkers (TNFa, MCP-1), and reduced nitrotyrosine, a plasma biomarker for oxidative stress. Rosiglitazone, atorvastatin, captopril, and TEMPOL were the most effective interventions at achieving these outcomes, with TEMPOL and atorvastatin having the largest benefit. Chronic treatment with L-NAME, conversely, resulted in the most severe manifestation of each vascular pathology. Temporal analysis of the interaction between NO bioavailability, vascular reactivity, and vascular stiffness reveal a progressive and concurrent decrease in each of these metrics from 7 to 17 weeks in OZR; animals from each treatment group fit neatly into the three-dimensional 
model, with the most efficacious treatments at preserving NO bioavailability trending towards values for LZR, while the least efficacious treatments trended towards values for untreated OZR. As was found in chapter 2 , the increase in oxidative stress and inflammation that occurs with the onset of MetSyn in OZR significantly impaired functional and structural components of the cerebral circulation.

The second and third chapters of this dissertation provide a substantial body of evidence that development of MetSyn leads to structural and functional impairments in the middle cerebral arteries, as well as a progressive loss of penetrating and parenchymal arterioles and capillaries within the cortex. The next step was to evaluate how these cerebrovascular impairments inherent in OZR would impact the pathophysiology of prevalent cerebrovascular diseases. Chapter 4 of this dissertation investigated the vascular and neurological outcomes to ischemic stroke in LZR and OZR, and attempted to elucidate distinct mechanisms that contribute to both the acute stroke injury as well as the long-term vascular complications from ischemic stroke.

Chapter 4 established that ischemic stroke in rats with pre-existing MetSyn increased infarct size and mortality rates, impaired recovery of cerebral blood flow following reperfusion, and caused a progressive exacerbation of stroke-induced vascular dysfunction in both hemispheres of the brain. While vascular reactivity was impaired in the ipsilateral (IL) MCA (stroke side MCA) of both LZR and OZR at 24 hours post-stroke, the dysfunction was greater in OZR. Two weeks post-stroke, reactivity remained constant in LZR IL MCA and contralateral (CL) MCA, and was improved in both by acute treatment with TEMPOL; in OZR IL MCA however, reactivity was completely eliminated, and vessels instead constricted to acetylcholine. Acute treatment with TEMPOL in OZR IL MCA prevented vasoconstriction, but did not 
improve dilation, as was seen in OZR CL MCA. These findings indicated that a specific injury, likely occurring during ischemia-reperfusion, was damaging the OZR IL MCA far worse than LZR IL or CL MCA, or OZR MCA. It was hypothesized that oxidative injury specific to the site of ischemia-reperfusion was the cause of this impairment. This hypothesis was tested by administering the $\mathrm{NAD}(\mathrm{P}) \mathrm{H}$ oxidase 2 (Nox2) inhibitory peptide gp91ds-tat during ischemic stroke in OZR. Treatment with gp91ds-tat was able to improve OZR IL MCA reactivity at 15 days post stroke to match OZR CL MCA, but had no effect on OZR CL MCA function. Nox2 inhibition also improved post-stroke cerebral blood flow, and eliminated infarction in the stroke territory. The conclusion of this experiment was that acute inhibition of Nox2, a highly expressed enzyme in leukocytes that respond to ischemic injury, could prevent oxidative damage to both the IL MCA and the ischemic core of the stroke during and immediately following an ischemic event.

Chapter 4 provided additional evidence that oxidative stress and ROS were contributing to the loss of NO bioavailability that defines cerebrovascular dysfunction in MetSyn. The fifth chapter of this dissertation sought to establish whether chronic stress/depression, which is known to impair peripheral vascular reactivity, would further exacerbate these pathologies. Additionally, chapter 5 investigated whether exercise training would prevent the onset of stress/depression induced vasculopathy in both LZR and OZR, and establish whether the antioxidant, anti-depressant effects of exercise would be sufficient to treat stress-induced depression in OZR.

The experiments in Chapter 5 involved imposing the unpredictable chronic mild stress (UCMS) protocol, a translationally-relevant model of environmental stressors that reproducibly induces depressive symptoms in rodents- on LZR and OZR for 8 weeks to induce depression and 
vascular dysfunction. Concurrently, a cohort of LZR and OZR received exercise training, or exercise training with UCMS, for the 8 week period. The main finding of this chapter is that UCMS induces vascular dysfunction in MCA of LZR and OZR, as well as eutrophic remodeling and cortical rarefaction in UCMS treated OZR. Acute incubation of vessels with TEMPOL and L-NAME revealed that these impairments largely stemmed from alterations in NO bioavailability, with impairments being substantially greater in OZR than LZR. Exercise training was able to improve each of these symptoms in both LZR and OZR. Interestingly, exercise trained animals from control OZR and UCMS OZR groups, as well as the UCMS LZR groups, could be characterized as 'good' or 'poor' responders to exercise training. 'Good' responding OZR showed a dramatic improvement in MCA reactivity, restoring reactivity to the same as LZR control, while 'poor' responders showed a much smaller, albeit significant, improvement over OZR control and OZR UCMS. A similar dichotomy was seen for the 'good' and 'poor' responders in the LZR. These findings suggest that the anti-depressive and vasculoprotective benefits of exercise training may be limited in many individuals. While the finding that exercise improves depressive symptoms and vascular health is not itself novel, this study was the first to conclusively link chronic stress/depression with cerebrovascular dysfunction, in lean as well as MetSyn animals, and the exercise component helps to establish the causative mechanisms through which these impairments occur and could subsequently be treated.

Each chapter of this dissertation used TEMPOL as a scavenger of ROS to reduce levels of superoxide and improve NO bioavailability, either acutely on ex vivo vessels, chronically through dietary administration, or both. TEMPOL is a mimetic of the enzyme superoxide dismutase (SOD) and therefore will work to directly reduce superoxide levels; SOD itself however turns superoxide into oxygen or hydrogen peroxide, which itself is a vasoactive 
metabolite that can contribute to vasodilation or potentially impact basal tone. In our experiments, acute incubation of vessels with TEMPOL did not result in any change in resting vessel diameter or tone. TEMPOL also had a negligible effect on reactivity in control LZR, exercise LZR, and exercise OZR, indicating that conditions of healthy NO bioavailability were significantly more important that the role of any SOD-produced peroxide at baseline or during vasodilation. However, a specific significant role of hydrogen peroxide cannot be completely ruled out by the methods presented in this dissertation, and is therefore a limitation of each study. Future studies could control for this by specifically treating with catalase to directly evaluate the role hydrogen peroxide plays in each of the pathways and conditions discussed here.

In conclusion, MetSyn in the OZR is associated with structural and functional impairments in the cerebrovasculature. These MetSyn-induced impairments are significantly driven by loss of NO bioavailability, and highly correlated with an increase in oxidative stress and inflammation. As a consequence of poor perfusion and control of cerebral blood flow, animals with MetSyn demonstrate significantly worse vascular and neurological outcomes to ischemic stroke, and are more severely affected by vascular impairments from chronic stress/depression. Treating underlying pathologies of MetSyn, such as hypertension, diabetes, and dyslipidemia, has limited efficacy at improving cerebrovascular function. Instead, therapies which focus on improving NO bioavailability by reducing inflammation and production of ROS are the most effective option for reducing cerebrovascular dysfunction in MetSyn.

There are many clinical implications of these findings. When considering the extremely high prevalence of MetSyn in the US and its link to diseases such as stroke, myocardial infarction, atherosclerosis, dementia, and Alzheimer's disease, it becomes necessary to question how the comorbid presence of MetSyn directly impacts the pathophysiology of these diseases. 
Incorporating MetSyn into preclinical models of these diseases may serve to enhance the translational utility of these models and improve the clinical relatability of their findings.

This dissertation provides preclinical evidence that targeting acute production of ROS during ischemic stroke may substantially improve vascular reactivity and reduce oxidative injury in the ischemic core, leading to better outcomes for stroke patients. Evidence is provided for efficacy of exercise as an anti-depressant and anti-oxidant therapy; however, mechanisms are also identified through which more targeted therapies can be designed, perhaps to capture 'exercise in pill form'. More broadly, this dissertation provides a wide body of evidence that severe, significant cerebrovascular dysfunction develops due to the underlying pathologies of the metabolic syndrome.

Clinically, cognitive function is not often considered when diagnosing and treating MetSyn and other forms of CVD; however, these impairments may be significant contributors to the pathological development of vascular cognitive impairments, vascular dementia, and loss of collateral reactivity that worsens outcomes in ischemic stroke. It is well-established that the damages and deficits from dementia and mild cognitive impairments are permanent, and cannot be reversed; the same goes for neuronal death in stroke. It is therefore of critical importance that cerebrovascular health is considered, and potentially treated, well in advance of the manifestation of cerebrovascular disease.

By reducing oxidant stress and improving NO bioavailability in the brain, healthy regulation of blood flow can be ensured for functional hyperemic responses generated by neurons, or for promoting collateral flow during cerebral ischemic events. While evaluating efficacy of therapeutics to achieve such benefits was not the primary aim of this dissertation, the results presented here show that drugs such as captopril, atorvastatin, and TEMPOL are effective 
at mitigated cerebrovascular dysfunction from MetSyn. Additionally, exercise was highly effective in both regular OZR and UCMS-treated OZR. Of course, TEMPOL is not viable for application in humans, and the compliance issues with prescribing exercise are well documented; nevertheless, the mechanisms through which these treatments confer benefits to the cerebrovasculature are defined within this dissertation, and future studies to identify more ideal compounds for human use would be warranted. The therapeutics studied here would therefore serve as a good starting place for the evaluation or development of novel compounds for treating cerebrovascular dysfunction.

There are several future directions this work could take. Firstly, further evaluation and characterization of gp91ds-tat as an acute inhibitor of Nox 2 during ischemic stroke is warranted and necessary to evaluate the clinical potential of this compound and this pathway for treating stroke in human patients. The preliminary data of the pilot study presented within this dissertation are promising, and offer a novel approach for limiting oxidative damage that occurs in both the cerebral vasculature and within the infarcted tissue during and after ischemic stroke. Dose-escalation should be conducted, as well as single vs multi-time point administration of gp91ds-tat. Additionally, other Nox family inhibitors should be evaluated for their therapeutic potential against gp91ds-tat specifically. Another immediate future direction for the work in this study is to identify and characterize the distinct pathways through which microvascular rarefaction occurs in the cerebrum. While it is likely that oxidative stress and reduced NO bioavailability are causal factors in this rarefaction, the pathways through which vessels are broken down and through which angiogenesis is regulated need to be characterized in order to better understand these processes and consider therapeutic approaches for mitigating or reversing cerebral rarefaction in patients. 
Long-term, this work has interesting implications for the study of MCI and dementia. While preclinical models are not necessarily adequate for studying age-related cognitive disorders, efforts could be undertaken to characterize vascular density in the brains of patients with age and in clinical conditions such as MCI and dementia. Imaging studies to determine overall basal metabolic activity of neurons could be performed to establish any relationships between blood flow perfusion and neuronal activity, and correlations sought after between impaired cerebral blood flow and the advancement of cognitive deficits and dementias. Additionally, long term augmentation of NO bioavailability should be studied in patients taking daily doses of drugs such as atorvastatin or captopril to determine whether they have lower risk of MCI or dementia independently of the direct beneficial effects of the drug on cholesterol or blood pressure, respectively. Such observational studies could offer insight as to the direct translational relevance of the mechanisms of cerebrovascular disease presented herein, and offer further justification for clinical trials to establish which means may be most effective at preserving cerebrovascular health and function in humans. 


\title{
An Unpredictable Chronic Mild Stress Protocol for Instigating Depressive Symptoms, Behavioral Changes and Negative Health Outcomes in Rodents
}

\author{
AUTHORS: \\ Frisbee, Jefferson C \\ Department of Physiology and Pharmacology \\ Center for Cardiovascular and Respiratory Sciences \\ West Virginia University Health Sciences Center \\ Morgantown, WV USA \\ jefrisbee@hsc.wvu.edu \\ Brooks, Steven D \\ Department of Physiology and Pharmacology \\ Center for Cardiovascular and Respiratory Sciences \\ West Virginia University Health Sciences Center \\ Morgantown, WV USA \\ Sbrooks8@mix.wvu.edu \\ Stanley, Shyla C \\ Department of Physiology and Pharmacology \\ Center for Cardiovascular and Respiratory Sciences \\ West Virginia University Health Sciences Center \\ Morgantown, WV USA \\ sstanle6@mix.wvu.edu
}

Alexandre C. d'Audiffret

Division of Vascular and Endovascular Surgery

Center for Cardiovascular and Respiratory Sciences

West Virginia University Health Sciences Center

Morgantown, WV USA

adaudiffret@hsc.wvu.edu

\section{CORRESPONDING AUTHOR:}

Frisbee, Jefferson C

Department of Physiology and Pharmacology

Center for Cardiovascular and Respiratory Sciences

West Virginia University Health Sciences Center

Morgantown, WV USA

jefrisbee@hsc.wvu.edu

Video (C) 2015 Journal of Visualized Experiments. Authors retain $@$ of written article.

KEYWORDS: Models of Clinical Depression, Models of Chronic Stress, cortisol, anhedonia, learned helplessness, behavioral impairments 


\section{SHORT ABSTRACT:}

The unpredictable chronic mild stress (UCMS) protocol is a validated method for studying behavioral and physiological changes associated with chronic stress and depressive symptoms. Eight weeks of imposition of the UCMS protocol induces behavioral changes and poor health outcomes in rodents of either gender.

\section{LONG ABSTRACT:}

Chronic, unresolved stress is a major risk factor for the development of clinical depression. While many preclinical models of stress-induced depression have been reported, the unpredictable chronic mild stress (UCMS) protocol is an established translationally-relevant model for inducing behavioral symptoms commonly associated with clinical depression, such as anhedonia, altered grooming behavior, and learned helplessness in rodents. The UCMS protocol also induces physiological (e.g., hypercortisolemia, hypertension) and neurological (e.g., anhedonia, learned helplessness) changes that are clinically associated with depression. Importantly, UCMS-induced depressive symptoms can be ameliorated through chronic, but not acute, treatment with common SSRIs. As such, the UCMS protocol offers many advantages over acute stress protocols or protocols that utilize more extreme stressors. Our protocol involves randomized, daily exposures to 7 distinct stressors: damp bedding, removal of bedding, cage tilt, alteration of light/dark cycles, social stresses, shallow water bath, and predator sounds/smells. By subjecting rodents 3-4 hours daily to these mild stressors for 8 weeks, we demonstrate both significant behavioral changes and poor health outcomes to the cardiovascular system. This approach allows for in-depth interrogation of the neurological, behavioral, and physiological alterations associated with chronic stress-induced depression, as well as for testing of new potential therapeutic agents or intervention strategies. 


\section{INTRODUCTION:}

Depressive mental illness is a complex neurological disorder that is currently recognized as a leading cause of disability and disease burden worldwide. The NIMH reports that approximately $12 \%$ of Americans suffer from clinical depression, with twice as many women affected versus men ${ }^{1}$. In the US alone, depression accounts for billions of dollars direct healthcare costs and an estimated $\$ 193$ billion more in indirect costs (lowered earnings and lost productivity $)^{2}$. Symptoms of depression include anhedonia, changes in weight and sleep cycles, decreased physical activity and personal hygiene, feelings of hopelessness or guilt, and/or reoccurring thoughts of death or suicide. During the last decade, epidemiologic and clinical studies have indicated that depression is an independent risk factor for cardiovascular disease (CVD) morbidity and mortality ${ }^{3}$, and is predictive of more severe prognosis of cardiovascular pathologies, including atherosclerosis, hypertension, myocardial infarction, and coronary artery disease, regardless of prior history of overt $\mathrm{CVD}^{4}$. Despite the increasing prevalence and adverse public health impact of depression, the etiology and related pathophysiology of this disease is poorly understood and the heterogeneity of the disorder due to various factors (e.g. genetic, biological, and environmental components) has made clinical diagnosis difficult to define.

Evidence indicates that irresolvable psychological stress is a major contributing factor for developing depressive illnesses and may also be a potent pathogenic factor linking depression and CVD, in part due to disruption and dysregulation of the hypothalamic-pituitary-adrenal (HPA) axis ${ }^{5,6}$. Dysfunction of the HPA axis is a major mechanism that has been linked to the behavioral and physiological changes observed in depression and the development of several

CVD risk factors, including dyslipidemia, obesity, and diabetes ${ }^{7}$. Multiple preclinical models of depression have been developed in attempt to replicate the mechanism of altered HPA axis 
activity characteristic of clinical depression; such models provide a validated means for investigating the behavioral, neurological, and physiological changes associated with chronic and acute stress in animals. The validity of an animal model of disease is based on the relevance of the etiology and progression of the model design and its ability to recapitulate anatomical, neurophysiological, and behavioral features observed in human disease. In addition, preclinical responses to treatments (such as SSRIs) should yield similar results to those observed in clinical settings.

Several animal models of stress-induced depression are currently utilized in research, such as learned helplessness, early life stress, and social defeat stress. However, each of these models has inherent disadvantages that lessen their translational efficacy ${ }^{8}$. Within the last decades, the Unpredictable Chronic Mild Stress (UCMS) protocol has emerged as one of the most translationally-relevant models for studying the pathophysiology of depression in rodents ${ }^{9}$. This model is based on the fundamental concept that chronic exposure to stressors disrupts stress response systems and ultimately leads to the development of depressive disorders. During the UCMS protocol, animals are exposed to a randomized series of mild environmental and social stressors on a daily basis. A crucial factor that enhances the relevance of this model to human situations lies in the high degree of unpredictability and uncontrollability of the stressors, as well as the time at which they are introduced. Additionally, the UCMS protocol uses only mild stressors, instead of relying on early life or aggressive physical stimuli. Over a period of UCMS exposure, depressive behaviors develop and are comparable to clinical symptoms, including decreased responsiveness to rewards (anhedonia), changes in physical activity and investigative behavior (helplessness and despair), deterioration of the coat state and altered sexual activity ${ }^{10}$. Almost all demonstrable symptoms of depression have been reported using this model, and 
studies have demonstrated that these behaviors persist for several weeks following the cessation of stress. In addition, these UCMS-induced depressive behaviors can gradually be revered by chronic, but not acute, treatment with particular antidepressants, suggesting similar neurological effects of therapeutic improvement that closely mirrors the clinical action and variable efficacy

of these agents in humans ${ }^{11,14-18}$. Here, we report a detailed description of the UCMS protocol and describe typical behavioral and vascular outcomes in mice.

\section{PROTOCOL:}

All procedures described below have been reviewed and approved by the Institutional Animal Care and Use Committee at the West Virginia University Health Sciences Center.

\section{Selection of animal model.}

NOTE: Most commonly used species in the UCMS model include Sprague Dawley, and Wistar rats and the BALB/cJ mouse; other murine models have shown limited efficacy (DBA/2, C57BL/6). However, careful consideration should be given to the particular mouse or rat strain of choice, as the genetic background and susceptibility to environmental stressors vary widely among species and have been shown to be critical factors affecting the outcomes of the study. ${ }^{11}$, 14, 15,19

1.1) The protocol takes at least 7-9 weeks to fully induce depressive symptoms, so animals of the appropriate age must be utilized (e.g. if the desired endpoint is to be evaluated in a 17-week old rat, then the rats must start the UCMS protocol at $\sim 9$ weeks of age). 
1.2) Single-house animals undergoing UCMS in IACUC-approved cages and facilities, with food and standard chow available ad libitum (unless otherwise required by specific protocol).

1.3) Take special care to ensure controls are housed in a quiet room with minimal activity to reduce effects of any possible unknown environmental stressors.

\section{Unpredictable Chronic Mild Stress Protocol.}

NOTE: Animals undergoing UCMS are transported daily ( $>5$ days per week) to a clean room used for UCMS manipulations. Animals are exposed to 1 or 2 of the stressors listed below each day. Stressors are performed on a randomized schedule, and each stress is administered for a minimum of 3-4 hours (see Table 1).

2.1) Dampen the bedding by pouring 10-20 oz. of clean water into each standard cage. Adjust the amount of water accordingly to the size of the cage in order to fully dampen the bedding but not to cause pooling of water. Reside the animals in damp cage for 3-4 hours.

2.2) Remove bedding from each cage for 3-4 hours, after which animals are transitioned into another stressor requiring an empty cage (shallow bath, cage tilt) or placed into a clean cage with fresh bedding. 
2.3) Tilt cages to approximately 45 degrees (without bedding) for 3-4 hours. Cages can be tilted with a sturdy object that will remain in place as the animal moves around in the cage.

2.4) Alter normal 12 hour/12 hour light/dark cycles into successions of 30 minute periods lasting for 8 hours. After, return animals to the normal cycle.

2.5) Transfer each animal from its home cage to that of the cage of a neighboring animal that has been removed for 3 hours.

2.6) Remove all bedding from each cage and add water to a depth of $\sim 0.25$ inches for mice or $\sim 0.5$ inches for rats for approximately 4 hours. Use water that is warmer than room temperature $\left(\sim 30^{\circ} \mathrm{C}\right)$ to minimize hypothermia potential. Briefly dry animals with a soft towel at the conclusion of the water exposure prior to placement into clean cages.

2.7) Expose animals to predator smells/sounds by adding randomly distributed sample tufts of fur or urine $(10-20 \mathrm{ml})$ from a natural predator species into the cage for 3-4 hours. Alternatively, play growling or predatory noises from natural predator species in close proximity to cages for 3 hours.

2.8) At the end of each daily stress period, place all animals into clean cages and return them to the housing facility. 


\section{Animal monitoring/grooming scores.}

NOTE: Each animal is monitored daily for development of lesions or wounds, and weekly for changes in weight and an overall grooming assessment.

3.1) Inspect each animal daily for the development of wounds or sores that may require veterinary treatment. Animals that develop mild injuries may continue in the UCMS protocol on the consent of the veterinarian.

3.2.1) Two investigators trained in evaluating coat grooming inspect each animal and assign a weekly coat score on a scale of $0-1$. This score is determined by assigning individual scores (0- clean, 1-dirty) to each of 8 body regions- head, neck, dorsal coat, ventral coat, tail, forelimbs, hind limbs, and genital region. The same investigators are to perform this task throughout the UCMS protocol to maintain consistency in assigning coat scores.

3.2.2) Any animal that experiences a sharp reduction in body weight (i.e., >15\%) should be reported to the veterinary staff and must be removed from the protocol.

\section{Behavioral testing and outcome measurements.}

NOTE: Several methods of assessment can be used to quantify behavioral changes from the UCMS protocol in rodents.

\section{1) Cumulative Coat Grooming Score.}

4.1)1. As described above, a weekly coat grooming score is assigned for each animal. 


\section{2) Sucrose Splash Test:}

4.2)1. Place the animal in a clean cage lined with an cotton pad or towel to prevent bedding from adhering to its coat. Spray a $10 \%$ sucrose solution onto the head and dorsal coat of each animal and record total grooming behavior for 5 minutes.

4.2)2. Grooming activity (defined as cleaning of the fur by licking or scratching) is measured by latency (idle time between the first spray and initiation of grooming) and frequency (number of times grooming a particular body part).

\section{3) Sucrose Preference Test:}

4.3)1. Habituate animals to drink $1 \%$ sucrose solution (w/v) 72 hours before the test and then provide exposure to two bottles (1\% sucrose solution versus tap water in different bottles). After habituation, provide animals with ad libitum access to sucrose solution and tap water for 3 hours. After $3 \mathrm{~h}$ record the consumption volumes of sucrose solution and tap water and calculate sucrose preference as: [(sucrose consumption)/(water consumption + sucrose consumption) $] \times 100$.

\section{4) Tail Suspension Test (Mice Only):}

4.4)1. Suspend mice by the tail from a stable rod ( $20 \mathrm{~cm}$ from the floor) using adhesive tape (2 cm from tip of tail). Record immobility time over 5 minutes. Mice are considered immobile only when they hang passively and completely motionless. 


\section{5) The Forced swim Test:}

4.5)1. Place animals in a container ( $15-20 \mathrm{~cm}$ diameter for mice; $\sim 55-60 \mathrm{~cm}$ diameter for rats) filled with water at a temperature of $26 \pm 0.5^{\circ} \mathrm{C}$. Record the latency to the first bout of mobility and the time spent immobile during the 5-min testing period. At the end of a test, place the wet animal in a holding cage on a heating pad $\left(30-35^{\circ} \mathrm{C}\right)$ with normal bedding covered by an absorbent paper towel until dry.

\section{6) Elevated Plus Maze:}

4.6)1. Allow each animal to explore the maze freely for $5 \mathrm{~min}$. Record total entries into each arm, and total time spent in each arm, according to a priori criteria $^{20}$.

\section{7) Locomotor Activity:}

4.7)1. Record activity levels, defined as overall movement, fine movements, rearing movements, and inactivity, for both acute (30 minute) and/or chronic (12 hour) time periods. Do this using the home cage or a novel environment, depending on the individual environment (although consistency should be maintained) in real time by an observer or post-hoc by replaying and analyzing camera footage.

\section{Expansion/Variation of UCMS protocol:}

5.1. Administer appropriate therapeutic agents or challenges to study their ability to either blunt the development of UCMS-induced outcomes (if initiated early) or to reverse severity of established UCMS-induced outcomes (if initiated late). This is dependent on the individual laboratory or protocol ${ }^{11}$. 


\section{Final outcome assessments:}

6.1. Following the conclusion of UCMS protocols and behavioral testing, utilize animals in subsequent experiments in a manner that addresses the specific hypotheses, aims or goals of the individual laboratory.

NOTE: As examples, this can include cardiovascular, inflammatory or endocrine responses to chronic stress. Alternatively, therapeutic interventions can be introduced at this point to evaluate their effectiveness and mechanistic underpinnings.

\section{REPRESENTATIVE RESULTS:}

An example of the schedule for one week following the UCMS procedure is shown in Table 1. Each week, stressors were randomized to prevent acclamation and to ensure unpredictability of the stress challenge each day.

Measures of Depressive like Behaviors: Following 8 weeks of UCMS, there were significant alterations to both behavioral and physiological outcomes in chronically stressed rodents relative to their non-stressed control strains. Development of depressive like symptoms following UCMS was assessment via coat score, sucrose splash test, and tail suspension (mice only; references 12 and 13). A progressive decline in coat grooming scores was observed in all UCMS animals (Figure 1, Panel A), and this marked degradation in the physical state of the coat suggests decreased grooming activity. This behavior may parallel the lack of motivation to or loss of interest in performing everyday tasks, such as the maintenance of minimal personal care. 
UCMS animals also demonstrated an increased latency to groom and reduced total grooming time in the sucrose splash test (Figure 1, Panels B and C). Increased time to initiate grooming behavior along with decreased time spent grooming is associated with reduced motivation and a decline in self stimulatory behavior, or reduced sensitivity to pleasure. This is representative of the core symptom of depression, anhedonia. In addition, there was a significant increase in the immobility time during the tail suspension test in mice. As stated previously, this behavior is a measure of behavioral despair with greater immobility representing a more severe depressive like symptom.

Measures of Physiological Changes: MAP can be significantly elevated as a result of the UCMS protocol, but this is not consistently observed (Table 1). A significant reduction in the dilator response to methacholine was observed in the aortic rings of UCMS BALB/cJ mice compared to controls (Figure 2). This increased shift towards blunted dilator responses is observed in clinical cases of increased risk for development of cardiovascular disease.

UCMS mice manifested increased plasma levels of cortisol, a marker of chronic physiological stress, and nitrotyrosine, a marker of chronic oxidative stress (Table 2).

Additionally, UCMS mice showed significantly elevated levels of the pro-inflammatory markers TNF- $\alpha$ and MCP-1. Together, these results suggest that stressed animals develop a pathophysiological disease profile following 8 weeks of UCMS (Table 2). 
Divergence with Sex: Recent evidence has suggested that there is a substantial quantitative divergence in the responses to UCMS between male and female mice (13). Specifically, data suggest that female mice, exposed to the identical UCMS protocol as age-matched males, develop more severe behavioral responses to the protocol than do males (Table 3 and Figure 3), with elevated levels of cortisol, nitrotyrosine and markers of inflammation.

However, despite this divergence between sexes following the UCMS protocol, the vasculopathy that develops as a result appears to be blunted in the female sex as compared to the males, strongly suggesting a superior maintenance of endothelial function, despite the compromised environment (Figure 4).

For Figures 1 and 3, results were obtained using the techniques described above, with the application of ANOVA and t-tests as appropriate. For Figures 2 and 4, results were obtained using concentration-response relationships in ex vivo aortic ring preparations. Curves were fit with a three parameter logistic equation, with ANOVA and t-tests as appropriate for the lower bounds of the curves. Please see references ${ }^{12,13}$ for details.

\section{FIGURE LEGENDS:}

Figure 1: Depressive Symptoms Following Eight Weeks of UCMS in Mice. Data are presented for coat status (Panel A), the latency (Panel B) and frequency (Panel C) of facial grooming following a $10 \%$ sucrose solution spray, and the total period of immobility during the tail suspension test (Panel D) for control and UCMS mice. * $\mathrm{p}<0.05$ versus control. Please see reference $^{12}$ for details. 
Figure 2. Vasodilator Responses to Methacholine from Mice Following Eight Weeks of UCMS. Data are presented for mice under control conditions (control lower bound $=10.3 \pm 2.4 \%$ ) and following eight weeks of the imposed UCMS protocol (UCMS lower bound=33.6 $\pm 4.4 \%$ ). * $\mathrm{p}<0.05$ versus responses in untreated vascular rings from control mice. Re-printed from reference ${ }^{12}$ with permission.

Figure 3. Differences in Depressive Symptoms Following Eight Weeks of UCMS in Male and Female Mice. Data are presented for coat status (A), the latency (B) and frequency (C) of facial grooming following a $10 \%$ sucrose solution spray, and the total period of immobility during the tail suspension test (D) for control and UCMS mice. ${ }^{*} \mathrm{p}<0.05$ versus Control in the sex; $\dagger$ $\mathrm{p}<0.05$ versus UCMS-Male. Re-printed from reference ${ }^{13}$ with permission.

Figure 4. Differences in Vasodilator Responses from Male and Female Mice Following Eight Weeks of UCMS. Dilator responses of aortic rings to increasing concentrations of methacholine (upper panel, A) and sodium nitroprusside (lower panel, B) from mice under control conditions and after eight weeks of UCMS. ${ }^{*} \mathrm{p}<0.05$ versus Control in the sex; $\uparrow \mathrm{p}<0.05$ versus UCMS-Male. Re-printed from reference ${ }^{13}$ with permission.

Table 1: Two sample weeks for imposition of unpredictable chronic mild stress (UCMS) protocol.

\begin{tabular}{|l|l|l|l|l|l|l|l|}
\hline & Sunday & Monday & Tuesday & Wednesday & Thursday & Friday & Saturday \\
\hline Stressor 1 & Alter & Social & No & Damp & Alter & Predator & Social \\
& Light/Dark & Stress & bedding & Bedding & light/dark & sounds & Stress \\
\hline
\end{tabular}




\begin{tabular}{|l|l|l|l|l|l|l|l|}
\hline Stressor 2 & $\begin{array}{l}\text { Alter } \\
\text { Light/Dark }\end{array}$ & Cage Tilt & $\begin{array}{l}\text { Shallow } \\
\text { Bath }\end{array}$ & Cage Tilt & $\begin{array}{l}\text { Social } \\
\text { Stress }\end{array}$ & $\begin{array}{l}\text { Shallow } \\
\text { Bath }\end{array}$ & \\
\hline
\end{tabular}

Table 2. Baseline characteristics between mouse groups under control conditions and following 8 weeks of UCMS. All mice are aged 17-18 weeks. ${ }^{*} \mathrm{p}<0.05$ versus control. Modified from reference 12 .

\begin{tabular}{|c|c|c|c|c|}
\hline & $\begin{array}{l}\text { Males } \\
(n=14)\end{array}$ & $\begin{array}{c}\text { Females } \\
\qquad(\mathbf{n}=9)\end{array}$ & $\begin{array}{l}\text { UCMS-Males } \\
\qquad(\mathrm{n}=12)\end{array}$ & $\begin{array}{l}\text { UCMS-Females } \\
\qquad(\mathrm{n}=12)\end{array}$ \\
\hline Mass (g) & $29 \pm 2$ & $28 \pm 3$ & $30 \pm 3$ & $29 \pm 4$ \\
\hline $\mathrm{MAP}(\mathrm{mmHg})$ & $87 \pm 4$ & $91 \pm 4$ & $94 \pm 5$ & $90 \pm 5$ \\
\hline Insulin $_{\text {plasmm }}(\mathrm{ng} / \mathrm{ml})$ & $1.1 \pm 0.3$ & $1.2 \pm 0.4$ & $4.1 \pm 0.7^{*}$ & $4.5 \pm 0.5^{*}$ \\
\hline Glucoseblood (mg/dl) & $82 \pm 7$ & $80 \pm 8$ & $94 \pm 8$ & $101 \pm 10$ \\
\hline Cholesterol $_{\text {plasma }}(\mathrm{mg} / \mathrm{dl})$ & $71 \pm 7$ & $68 \pm 8$ & $78 \pm 6$ & $77 \pm 10$ \\
\hline Triglycerides $_{\text {plasm }}(\mathrm{mg} / \mathrm{dl})$ & $94 \pm 6$ & $101 \pm 8$ & $109 \pm 8$ & $116 \pm 12$ \\
\hline Nitrotyrosine $_{\text {plasma }}(\mathrm{ng} / \mathrm{ml})$ & $12 \pm 3$ & $11 \pm 4$ & $29 \pm 5^{*}$ & $36 \pm 6^{*}$ \\
\hline Cortisol plasma $_{(\mathrm{pg} / \mathrm{ml})}$ & $12 \pm 3$ & $14 \pm 4$ & $29 \pm 5^{*}$ & $44 \pm 4^{*}$ \\
\hline TNF- $\alpha_{\text {plasma }}(\mathrm{pg} / \mathrm{ml})$ & $2.3 \pm 0.3$ & $2.0 \pm 0.2$ & $4.1 \pm 0.4^{*}$ & $6.4 \pm 0.4 * \dagger$ \\
\hline MCP-1 $1_{\text {plasma }}(\mathrm{pg} / \mathrm{ml})$ & $2.8 \pm 0.3$ & $3.3 \pm 0.5$ & $10.2 \pm 1.0^{*}$ & $14.8 \pm 1.3^{*} \dagger$ \\
\hline
\end{tabular}

\section{DISCUSSION:}

Extensive clinical and epidemiological evidence has indicated that chronic stress is one of the most potent precipitating factors for depression. Repeated exposure to stressors may potentiate individual vulnerability to depression and other neuropsychiatric disorders as a 
consequence of the psychological and physical demands within the body that accumulate over time. The use of validated preclinical animal models offers a valuable translational tool for studying depression and comorbid diseases. Firstly, an ideal animal model offers an opportunity to understand molecular, genetic and epigenetic factors that may lead to depression. By using such models, the underlying mechanisms and the causal relationship between genetic or environmental alterations and depression can be investigated, potentially allowing a more thorough insight into the pathophysiology of depression. Secondly, validated, improved models of depression are an indispensable tool for identifying and evaluating novel therapeutics and treatments, an essential first step towards developing improved diagnostic and treatment options.

For many years, the UCMS model has been validated for use in psychological and behavioral studies as a translationally relevant rodent model for chronic stress, human depression and depressive symptoms, as it does an excellent job at reproducing clinical symptoms of human depression, including anhedonia and learned helplessness ${ }^{21}$. In addition, the temporal development of the depressive symptoms is also relevant for human disease conditions, as animals experiencing UCMS develop depressive symptoms over time in response to stress, which is considered to be more translationally relevant. The representative results reported here showcase the ability of this model to generate a symptomatic profile of depression, including both behavioral and physiological changes, in both a rat and mouse model. In addition, the severity of behavioral and physiological outcomes shows sex-specific differences that could potentially be used to study the pathological effects of stress on men and women ${ }^{21}$.

It should be pointed out that the outcome of stress exposure is influenced by several variables under experimental conditions, including the nature of the stress (physical/systemic vs. 
cognitive/psychological), the severity of the stress, and exposure parameters. Different techniques can be used to measure depression-like behaviors and physiological outcomes and

vary results among investigators ${ }^{21}$. For example, evaluation of the end points of behavioral tests may be variable depending on the use of recording methods as well as inconsistent methodology and bias amongst individual investigators. The particular strain/species used is also critical, as they have been shown to possess differences in susceptibility to UCMS. Further, other issues, such as the degree of control an animal has over stress exposure and the predictability of the stressor have been demonstrated to be important to the outcomes of the UCMS model, as greater unpredictability can reduce the probability of adaptive processes occurring upon repeated stress exposure and promote the appearance of stress effects on physiological and behavioral functions $^{21}$.

\section{ACKNOWLEDGMENTS:}

This study was supported by the American Heart Association (IRG 14330015, PRE 16850005, PRE 20380386, EIA 0740129N), and the National Institutes of Health (RR 2865AR; P20 RR 016477).

\section{DISCLOSURES:}

The authors have nothing to disclose. 


\section{REFERENCES}

1. NIMH Statistical Pages (Depression; Prevalence).

http://www.nimh.nih.gov/health/statistics/prevalence/major-depression-among-adults.shtml (Accessed 12/26/2014)

2. NIMH Statistical pages (Depression; Costs).

http://www.nimh.nih.gov/health/statistics/cost/index.shtml (Accessed 12/26/2014)

3. Lett, H. S., et al. Depression as a risk factor for coronary artery disease: evidence, mechanisms, and treatment. Psychosom Med, 66(3), 305-315, doi: 10.1097/01.psy.0000126207.43307.c0 (2004).

4. Plante, G. E. Depression and cardiovascular disease: a reciprocal relationship. Metabolism, 54(5 Suppl 1), 45-48, doi:10.1016/j.metabol.2005.01.013 (2005).

5. Pizzi, C., Manzoli, L., Mancini, S., Bedetti, G., Fontana, F., \& Costa, G. M. Autonomic nervous system, inflammation and preclinical carotid atherosclerosis in depressed subjects with coronary risk factors. Atherosclerosis, 212(1), 292-298, doi:

10.1016/j.atherosclerosis.2010.04.038. (2010).

6. Barden, N. Implication of the hypothalamic-pituitary-adrenal axis in the physiopathology of depression. J Psychiatry Neurosci, 29(3), 185-193, (2004).

7. Bowman, R. E., Beck, K. D., \& Luine, V. N. Chronic stress effects on memory: sex differences in performance and monoaminergic activity. Horm Behav, 43(1), 48-59, doi:10.1016/S0018-506X(02)00022-3 (2003).

8. Baune B.T., et al. The relationship between subtypes of depression and cardiovascular disease: a systematic review of biological models. Transl Psychiatry. 2:e92 doi: 10.1038/tp.2012.18. (2012).

9. O'Leary O.F., Cryan J.F. Towards translational rodent models of depression. Cell Tissue Res. 354(1):141-53, (2013). doi: 10.1007/s00441-013-1587-9.

10. Mineur, Y.S., Belzung, C., Crusio, W.E. Effects of unpredictable chronic mild stress on anxiety and depression-like behavior in mice. Behav Brain Res. 175(1):43-50, doi:10.1016/j.bbr.2006.07.029 (2006).

11. Yalcin. I., Belzung, C., Surget, A. Mouse strain differences in the unpredictable chronic mild stress: a four-antidepressant survey. Behav Brain Res. 193(1):140-3, (2008). doi: 10.1016/j.bbr.2008.04.021. 
12. d'Audiffret, A.C., Frisbee, S.J., Stapleton, P.A., Goodwill, A.G., Isingrini, E., Frisbee, J.C. Depressive behavior and vascular dysfunction: a link between clinical depression and vascular disease? J Appl Physiol, 108(5):1041-51, doi: 10.1152/japplphysiol.01440.2009. (2010).

13. Stanley, S.C., Brooks, S.D., Butcher, J.T., d'Audiffret, A.C., Frisbee, S.J., Frisbee, J.C. Protective effect of sex on chronic stress- and depressive behavior-induced vascular dysfunction in BALB/cJ mice. J Appl Physiol, 117(9):959-70, doi:

10.1152/japplphysiol.00537.2014 (2014).

14. Ibarguen-Vargas, Y., Surget, A., Touma, C., Palme, R., Belzung, C. Multifaceted strainspecific effects in a mouse model of depression and of antidepressant reversal.

Psychoneuroendocrinology. 33(10):1357-68, doi: 10.1016/j.psyneuen.2008.07.010 (2008).

15. Dalla, C., Pitychoutis, P.M., Kokras, N., Papadopoulou-Daifoti, Z. Sex differences in animal models of depression and antidepressant response. Basic Clin Pharmacol Toxicol. 106(3):226-33, doi: 10.1111/j.1742-7843.2009.00516.x (2010).

16. Mutlu, O., Gumuslu, E., Ulak, G., Celikyurt, I.K., Kokturk, S., Kır, H.M., Akar, F., Erden, F. Effects of fluoxetine, tianeptine and olanzapine on unpredictable chronic mild stress-induced depression-like behavior in mice. Life Sci. 91(25-26):1252-62, doi: 10.1016/j.lfs.2012.09.023 (2012).

17. Gumuslu, E., Mutlu, O., Sunnetci, D., Ulak, G., Celikyurt, I.K., Cine, N., Akar, F. The effects of tianeptine, olanzapine and fluoxetine on the cognitive behaviors of unpredictable chronic mild stress-exposed mice. Drug Res (Stuttg). 63(10):532-9, doi: 10.1055/s-00331347237 (2013).

18. Isingrini, E., Belzung, C., Freslon, J.L., Machet, M.C., Camus, V. Fluoxetine effect on aortic nitric oxide-dependent vasorelaxation in the unpredictable chronic mild stress model of depression in mice. Psychosom Med. 74(1):63-72, doi: 10.1097/PSY.0b013e31823a43e0 (2012).

19. Ripoll, N., David, D.J., Dailly, E., Hascoët, M., Bourin, M. Antidepressant-like effects in various mice strains in the tail suspension test. Behav Brain Res. 143(2):193-200, doi:10.1016/S0166-4328(03)00034-2 (2003).

20. Komada, M., Takao, K., Miyakawa, T. Elevated plus maze for mice. J Vis Exp. (22). pii: 1088, doi: 10.3791/1088 (2008). 
21. Golbidi, S., Frisbee, J.C., Laher, I. Chronic stress impacts the cardiovascular system: animal models and clinical outcomes. Am. J. Physiol. Heart Circ. Physiol. (in press), 2015
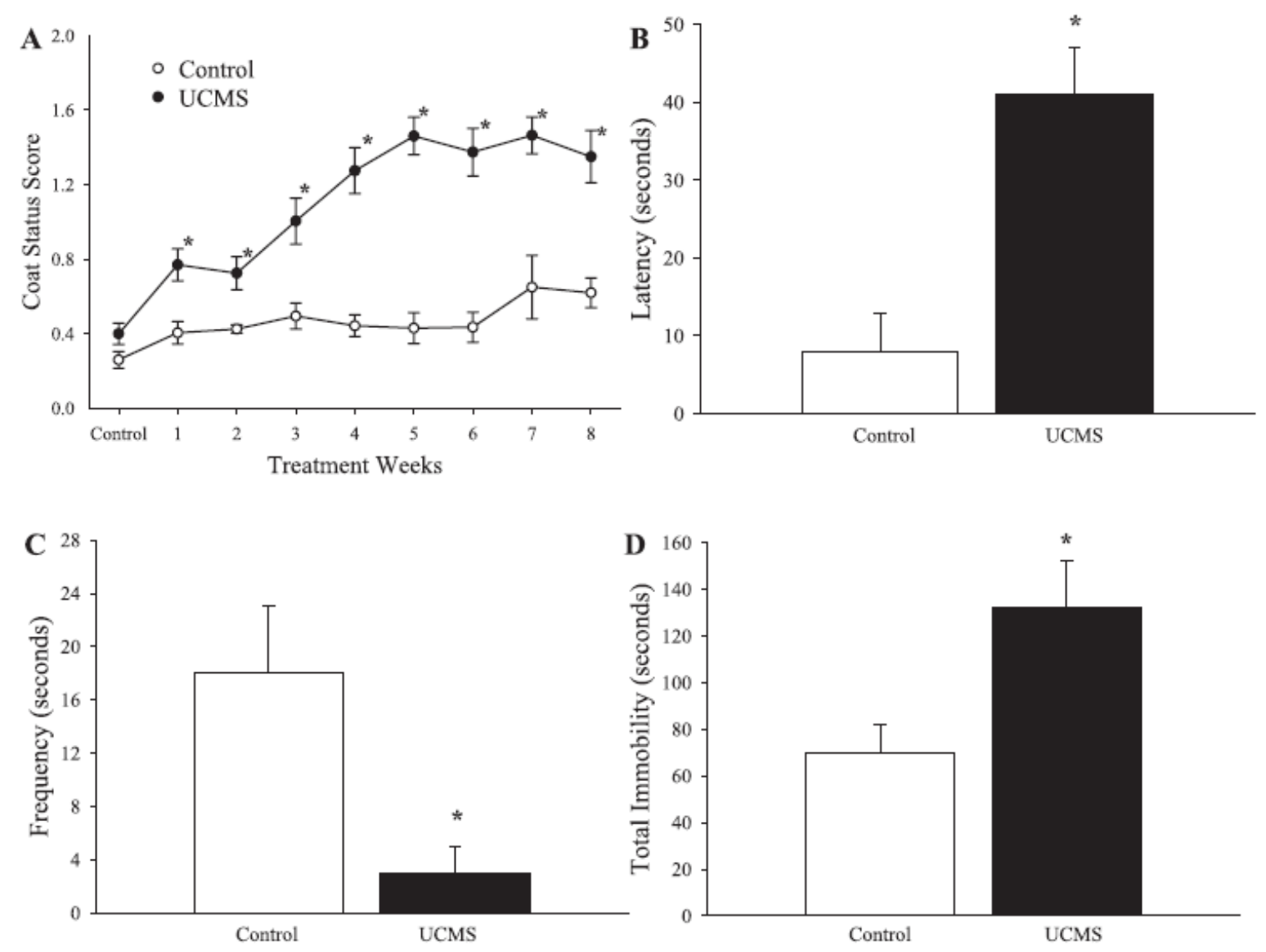

Figure 1.

Frisbee et al. 


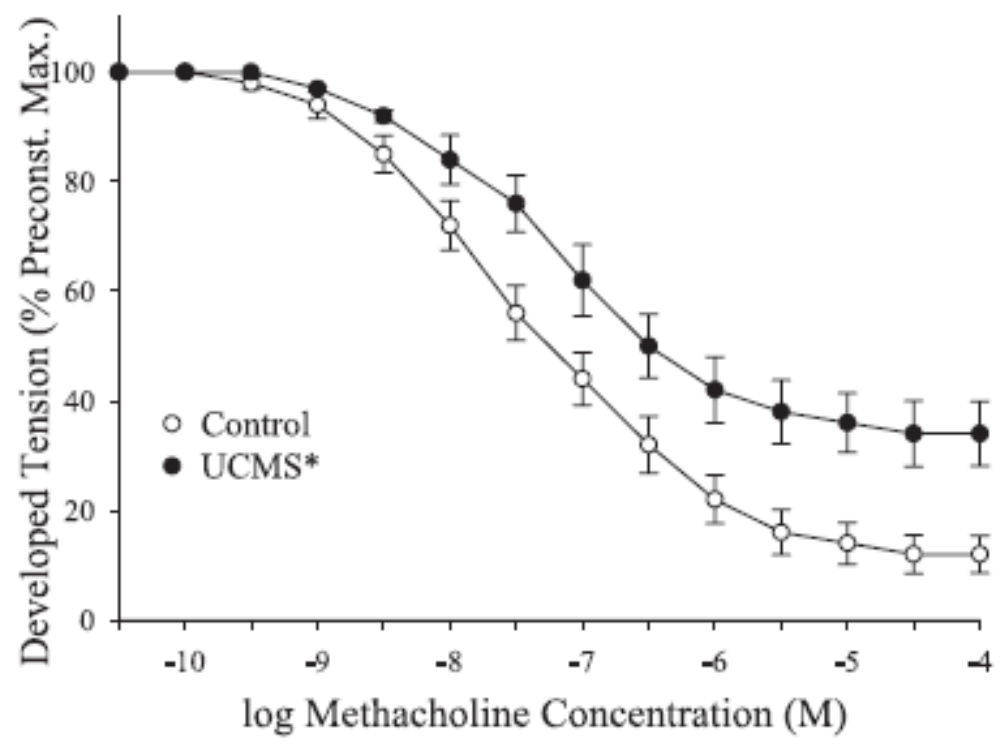

Figure 2.

Frisbee et al. 

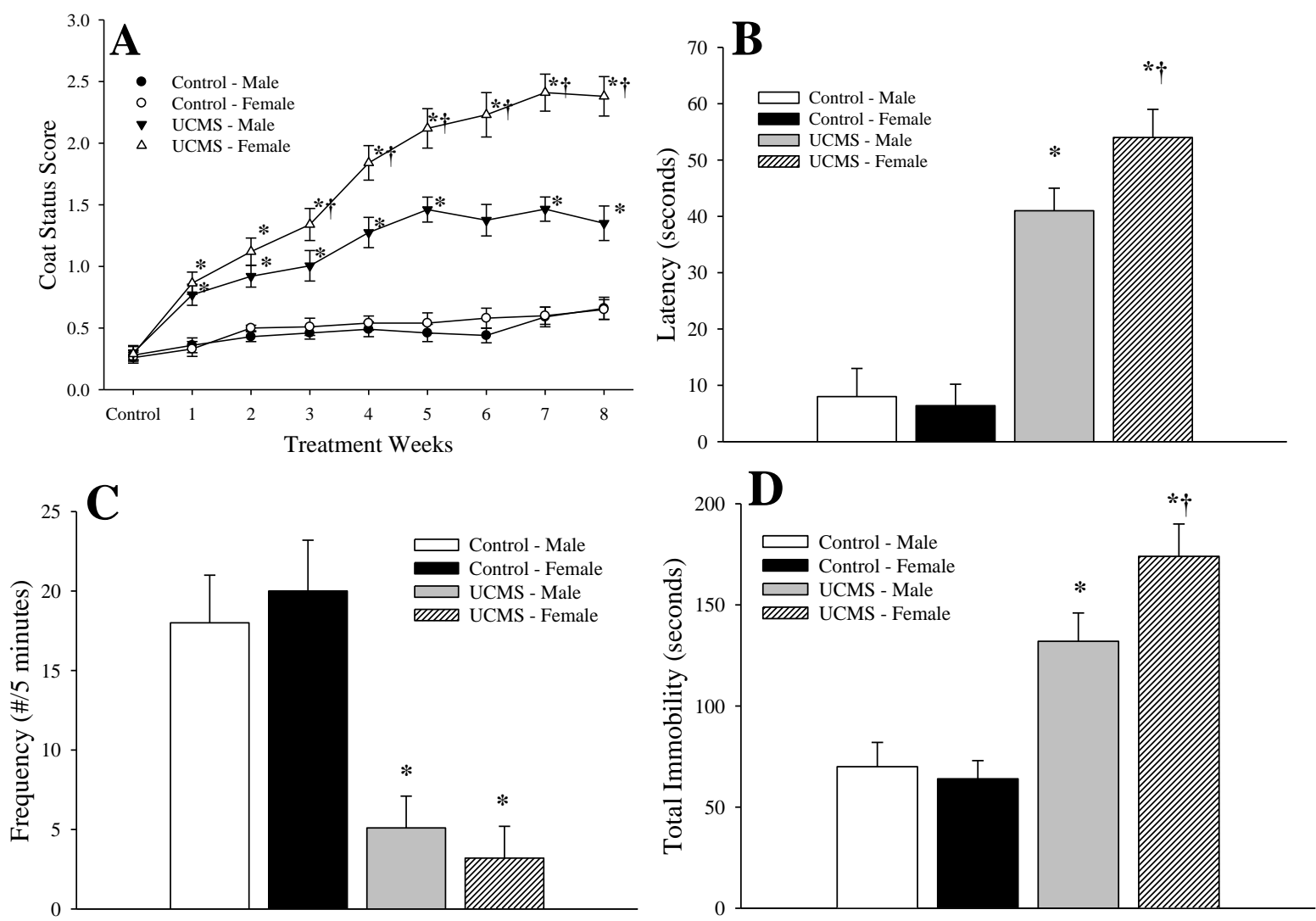

Figure 3.

Frisbee et al. 

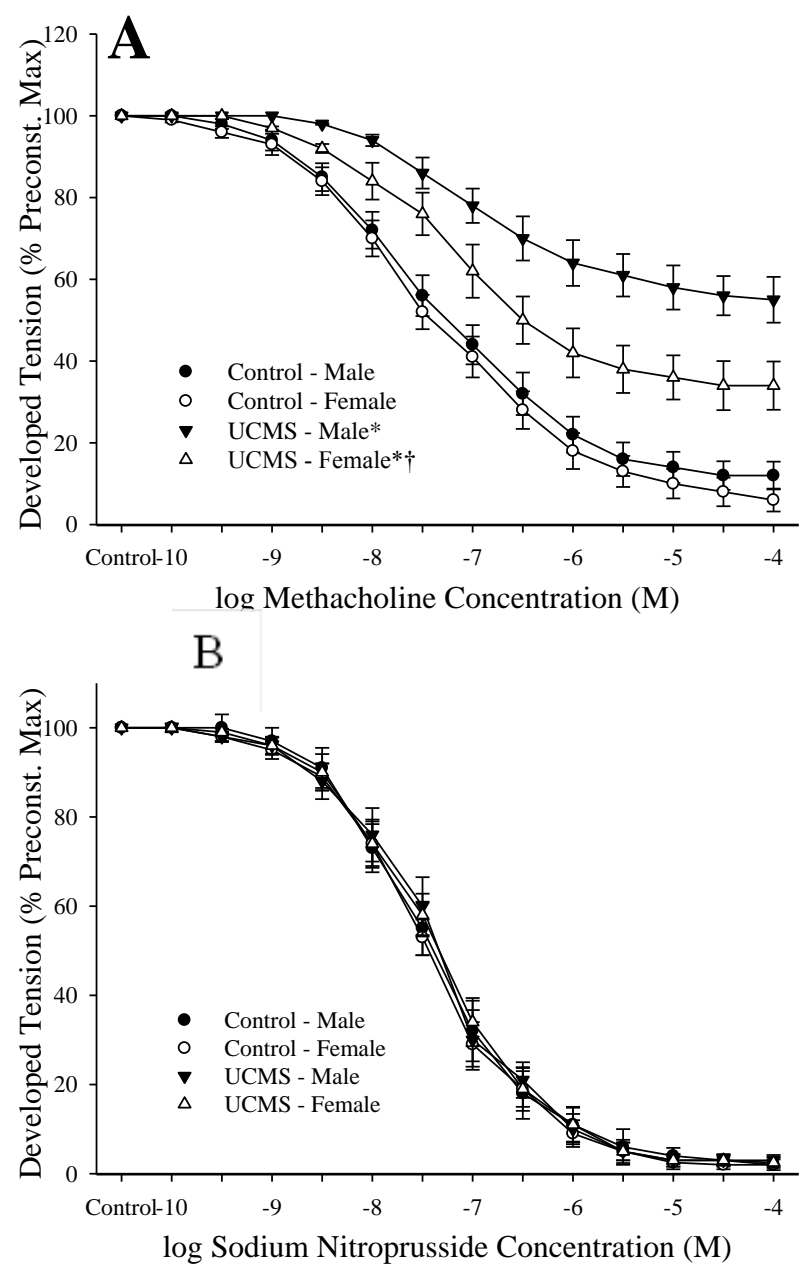

Figure 4.

Frisbee et al. 